


\section{Margins Exposed}

La expresión de La Comuna 13

By Mariana Restrepo Sierra

A 120-point thesis submitted to Victoria University of Wellington

Victoria University Faculty of Architecture and Design

2020

Word count : 21,967 



\section{ACKNOWLEDGEMENTS}

Agradecimientos

Thank you to Philippe for being such a supportive guiding light throughout this research. It has been a strange year and your patience and encouragement (even through Zoom calls) was appreciated.

To my parents for never letting me forget where I am from. For being a constant reminder of the brightness and beauty of our culture. For helping me to always believe in myself. Los quiero con todo mi corazon.

To Sophie; we've been in this together for 5 years and I cannot thank you enough for all the laughs and the tears but most of all the constant support.

To everyone I haven't named. You know who you are. Thank you from the bottom of my heart.

And last but absolutely not least, to my people in Colombia. Thank you for your colour, your passion and the love. This is for you. 


\section{PERSONAL STATEMENT}

Declaración personal

This piece of work is special; it is meaningful and it is personal.

I decided to embark on this research because I thought that nothing could make more sense than basing my thesis work on my home country, on my people. I was born in Medellin, Colombia and the love and passion that I feel for my country is something which will never waiver, no matter how much time I spend away from home. Colombia is a country full of colour, passion, culture and love. The community examined in this research, called La Comuna 13 is one which has experienced the absolute worst of our country's violent history and yet, has created something beautiful. The people that make this community have showcased the resilience and the strength of our culture. They have used art to document their pain but also to decorate their streets. Their creativity is a true reflection of the beauty of my culture and my country. In a smaller fashion, the work endeavoured in this thesis is as much for my own personal reconnection as it is to honour the many splendid displays of our colourful culture being created by the people of La Comuna 13. 


\section{ABSTRACT}

\section{Resumen}

In many countries, slum housing begins with the primary intention to house marginal communities and develop to embody a saturation of culture. From this saturation of culture emerges a new experience of architecture. Things such as visual art appear and directly interact with the architecture's facades and materiality. Such a site exists in Medellin, Colombia; it is called 'La Comuna 13'. Here, the architecture is made up of poorly constructed brick houses, most of which are now covered with street art created by the community members. Each one of the murals has been painted in order to record a particular and significant event in this community's painful and turbulent past. In fact, the community members have created a way to archive their history through an artistic expression manifested as a new layer added onto an architecture.

This research looks to create a framework where the practice of interior architecture can start to respond to such artistic expression. It endeavours to develop a process of re-coding the community generated art in order to create different interior programmes and designs. This thesis proposes that this can be achieved by a close examination of street art and by breaking it down into ideas such as layering, place-identity and memory in order to derive an architecture from it. The process includes the use of various iterative formats. The design of interior architecture has not yet been approached from the perspective of allowing art to be the design driver and form generator. This will produce an interior architecture which not only responds to an artistic and cultural expression but also helps to enable this expression. The iterations form a set a different engagement with society. As such they include an urban provocation, an orchestrated invitation and a discrete retreat.

One may argue that the proposed architecture has a motivation in exposing the recording of the trauma experienced by a community on the margins of a society onto the mainstream cultural space. 


\section{CONTENTS}

CONTENIDO

Acknowledgements $\quad$ i

Personal Statement

Abstract

Introduction 01

Context 05

Chapter 1: The Community 11

Chapter 2: The Ideas 17

- Memory 19

- Place Identity 20

- Art + Architecture 21

- Layering 22

Chapter 3: The Wall 25

Chapter 4: The Art 33

Chapter 5: The Encounters 49

1. Forced Encounter 51

2. Invited Encounter 79

3. Hidden Encounter 117

Chapter 6: The Development 159

1. House 161

2. Community Center 203

3. Inter-floor communal space 223

Conclusions 235

Final Reflections 237

Bibliography 239

Figure List 241 


\section{INTRODUCTION}

Introducción

Some of the most beautiful things are often found in the dark, hard-to-reach corners that most people are afraid to wander into. Often times treasures are hidden by a veil of darkness and scary stories; pushed to those outer corners and only heard of in whispers and legends. This is the case of a community in Medellin, Colombia who go by the name of La Comuna 13. A community with a dark past who have been pushed to the outskirts of the city as if damaged. From being marginalised and having so many memories and histories to tell began to sprout an artistic expression. This community started finding a way to display what they had experienced through murals which covered the walls in their streets. They began to reflect the beautiful and colour aspects of their culture; our culture. Being from Colombia, this design led-research is very personal and it originated from the realisation that in the city of Medellin, marginalised communities were not being expressed in the city centre. The aim became display the beauty that can come from a marginalised community but also how this beauty can be translated into an architecture which not only uses this art as a design driver but becomes an enabler for this artistic expression. This becomes relevant because it could open a door to allowing the expression of not only my culture, but of any other culture to be translated in this way. It could be a new way for interior architects to explore the way in which we design and the outcomes which can be achieved when art is at the forefront of the design process.

This becomes a matter not of appropriation, but of re-coding this art. Using it as a source of inspiration to derive form, materials and colours whilst not taking away the meanings and the memories attached to each mural.

This is the original intention of this design-led research. In order to engage in this endeavour, the author has devised the research in different sequential parts that can be seen in the methodology diagram (page 03) as a visual expression of the research process.

This design led research will first establish the context of the city (Medellin), of La Comuna 13 within the city and then also of the selected sites for the explorations carried out. Once the context of La commune 13 is established, concepts relevant to the research will need to be explored.

Chapter 2 includes Memory, Place identity, layering, art and architecture. There memory is explored in terms of the representation of memories through the painting of the murals. Place and identity becoming relevant themes surrounding La Comuna 13, both are tightly linked.

The notion of layering is relevant to not only the physical layering of the murals onto the façade of the architecture. But it is also significant on the layering of the memories from the members of La Comuna 13 being layered upon one another.

Chapter 3 focuses on the wall, an architectural object which in this specific instance acts as a canvas. The wall as the canvas is briefly visited through both literature and a series of diagrammatic studies.

In chapter 4, La Comuna 13 is introduced with both its background as well as a sample of some of the murals which can be found on their streets.

Finally, the three design iterations will be developed in the following chapters. They are formed by a series of intuitive exercises for re-coding the art as well as diagrammatic studies and, in one case, a photographic study. Each receive a reflection focused on the success of the design as well as future developments.

\section{RESEARCH QUESTION:}

What is the role of the interior architect in enabling the expression of the margins in the heart of the city?

How can an interior intervention enable the expression of a community in the margins of society throughout the urban fabric of mainstream culture? 


\section{METHODOLOGY}

\author{
Metodología
}

\section{AIMS}

The principal aims of this design-led research are to investigate how an interior intervention can:

- Become a response to the artistic expression of a community (In this case La Comuna 13)

- Facilitate different forms of artistic expression through its materiality and spatial qualities

- Reflect aspects of ideas such as layering, memory and place-identity within interior architecture

\section{OBJECTIVES}

The principal objectives of this design-led research investigation are to develop a framework for the design of an interior architecture that:

- Fulfils the spatial requirements of various programmes by actively engaging the proportions of the body and the needs of humans

- Acknowledges the culture of a community by facilitating their artistic expression but also taking aspects of the art to drive the design process

- Incorporates ideas of layering, place-identity and memory which are evident in this community by allowing these themes to influence the form, atmosphere and materiality of the design.
This thesis employs iterative design-led research testing, by using a combination of analogue drawing and digital drawing, as the dominant methodologies. Using this approach to design allows the research to build its argument iteratively and progressively by the added layering of each exploration and finding. It allows for a more methodical breakdown of the art being studied through the potential to utilise one process to begin with and then layer in the other in order to create a more enriched and layered process of drawing. It also means that the findings from each iteration can then inform the next and create a system of constant feedback throughout the design. These drawing techniques being the main devices used for concept generation throughout the design process as they allow for a visual interpretation of the research which can then be translated into the design of the interior architecture.

The use of literature reviews and precedent reviews is the other key element of the research methodology. Literature and precedents heavily inform the beginning stages of the research. Combining the findings from these processes with the previously mentioned methods of analogue drawings and digital drawings is another form of concept generation which allows the designer to interpret literary findings into architectural concepts. Literature and precedent reviews also serve to establish parameters and themes which are key to the design.

Another significant process incorporated is diagramming. Different to drawing in the sense that it offers a much more iterative and communicative approach to design exploration. This makes it an integral part of the research as it allows for the ease of communication of various concepts or ideas in a way in which they are easily understood. Creating a series of diagrammatic explorations to convey the development or exploration of various ideas throughout the research. It adds a layer to the process of drawing or literary reviews as it has the power to graphically depict ideas and concepts within both in a sequential and iterative manner.

And lastly, the use of digital modelling. This is a process which is incorporated throughout the entirety of the design process as it allows for a more spatial experience of what is being designed. The designer has the ability to explore the design in the three dimensional realm and gain a better understanding of materiality, atmosphere and space. 


\section{METHODOLOGY}

STRUCTURE + CONTENTS + PROCESSES

\section{CHAPTER1}

\section{WEEKS}

History of 'La Comuna 13'

Origins + Reasons + People
DRAWING ANALYSIS

(Interpretation)

\section{CHAPTER 2}

\section{WEEKS}

Architecture facilitating art

Memory through architecture

Histories through architecture

Layering Architecture
PRELIMINARY INDESIGN

$$
\text { LAYOUT }
$$

(Initial Document Structure + Chapter Title Page Layout + Page Layout))

\section{CHAPTER 3}

\section{WEEK}

The Wall

A diagrammatic + literary study of the role, function and interaction of the wall

Diagrammatic Study

\section{CHAPTER 4}

\section{WEEKS}

Introduction of the art

Analysis of the art Interpretative drawings to derive form for the interior architecture from the street art

The Extraction

(Intuitive exercise of shape extraction fro the art) -

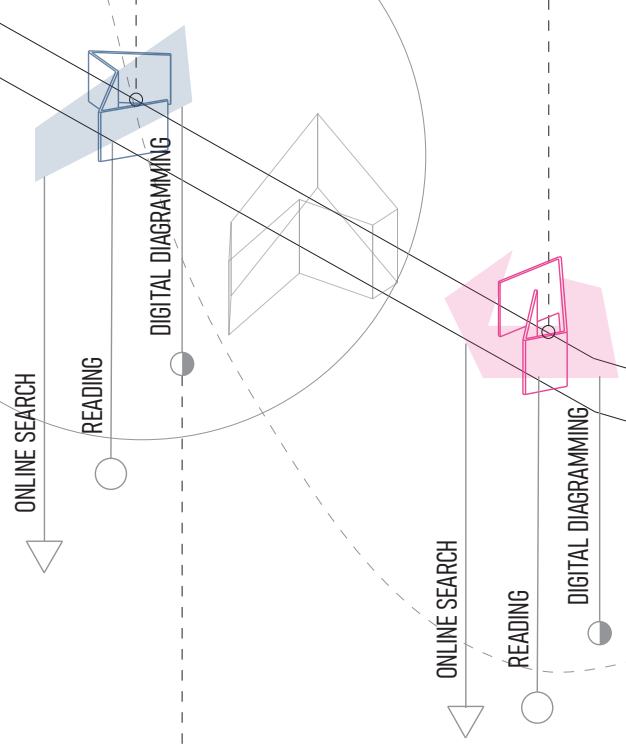

\section{PROCESSES}

PROCESSES EXPECTED TO BE USED + WHEN THEY WILL BE USED

\section{KEY}

$\triangle$ ONLINE SEARCH

$\checkmark$ PRECEDENT STUDY PENCIL DRAWINGS

- DIGITAL MODEL - DIGITAL DRAWING

- DIGITAL DIAGRAM

READING

PENCIL DIAGRAMS

RENDERING 


\section{CHAPTER 5}

\section{- - CONCEPT 1: URBAN INTERIOR}

5 WEEKS

\section{Precedent Study}

Initial Concept Development

(Drawings + Plans + Aesthetics + Atmosphere)

Site

(Location Map + Design on Site)

Final Imagery

\section{CONCEPT 2: DANCE STUDIO} 6 WEEKS

Relationship between dance + interior Precedent Study

Dancer Photographic Study

Initial Concept Development (Drawings + Plans + Aesthetics + Atmosphere)

Site

(Location Map + Design on Site)

Final Imagery
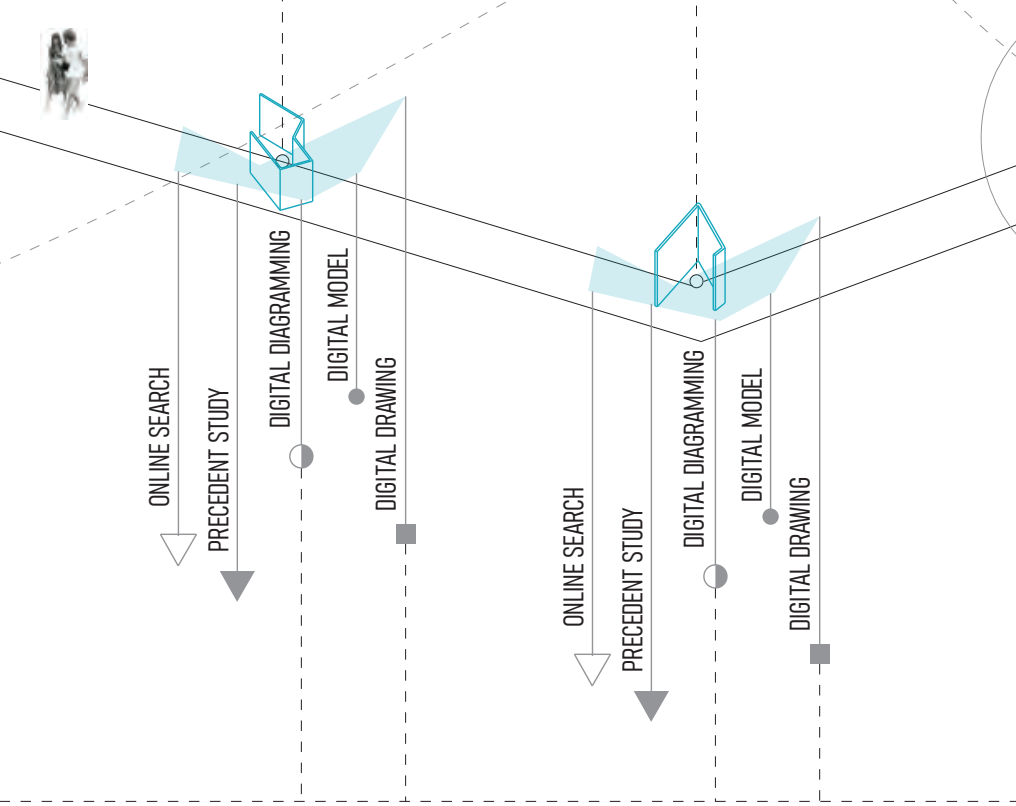

- CONCEPT 3: HIGH-DENSITY

\section{HOUSING}

6 WEEKS

High Density Housing

Adaptive Re-use

Housing + Family life in Colombia

Precedent Study

Host Building

(Plans + Analysis of Spaces)

Initial Concept Development

(Plans + Aesthetics + Atmosphere)

Site

(Location Map + Design on Site)

Final Imagery

T

\section{CHAPTER 6}

\section{DEVELOPED DESIGN}

\section{WEEKS}

The Layering

(Overlaying the interpretative drawings on top of plans)

Evolution of Floor Plans

Final Floor Plan Selected

Critical Analysis + Evaluation of others

Development of Chosen Concept Floor Plan (Lighting + Materiality + Furniture + Atmosphere)

Final Imagery

(Plans + Renders + Drawings)

Conclusions / Closing Statements

FINAL INDESIGN LAYOUT

6 WEEKS

Document Structure + Content Finalized
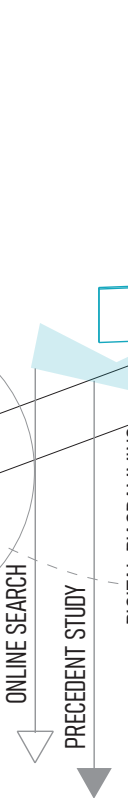

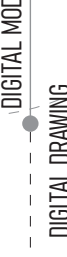
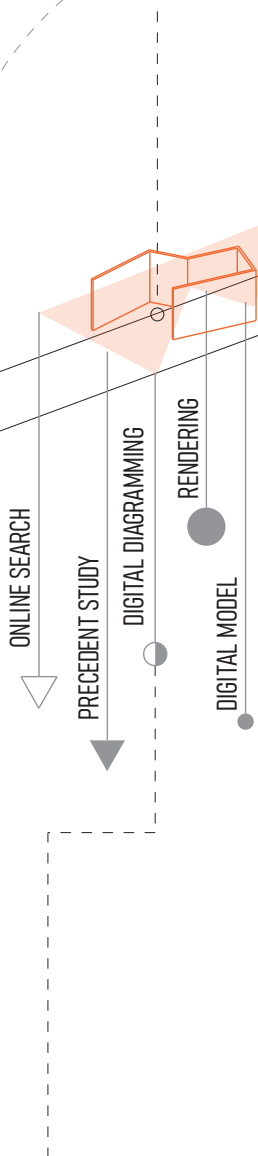
Contexto 
0 


\section{ECUADOR}

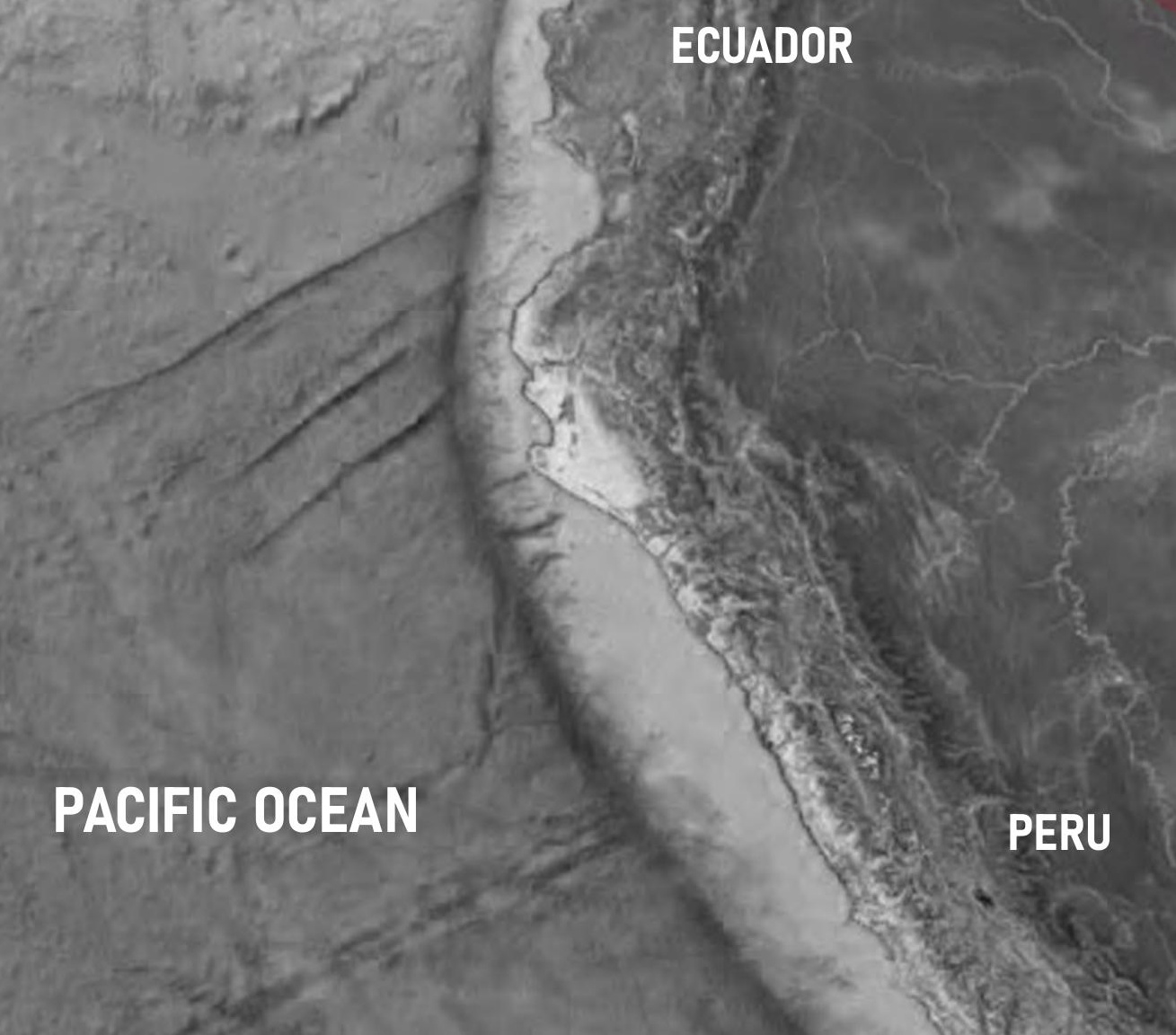

\section{CONTEXT TIER 01:}

STATE OF

AMAZONAS
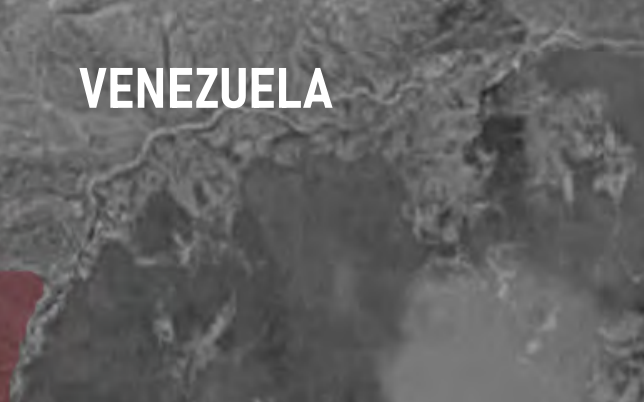

की

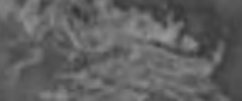
THE COUNTRY COLOMBIA WITHIN SOUTH AMERICA 


\section{MEDELLIN WITHIN COLOMBIA}





\section{CHAPTER 1: THE COMMUNITY}

Capítulo 1: La Comunidad 
01 


\section{LA COMUNA 13}

This content is unavailable.

Please consult the figure list for further details.

Figure 1

'La Comuna 13' is a high-density housing community which exists in Medellin, Colombia. It is located in Zone 4 of the city, along the western fringes. This site on the hilly edges spans 74.2 kilometres squared and is populated by 134,000 people, being one of the most densely populated areas of the city (Drummond et al., 2012). Here, the architecture is made up of poorly constructed houses using materials such as aluminium, wood and bricks. In this urban environment brightly coloured and carefully crafted images can be found on almost every building's walls. These images are the community's main source of cultural expression. It is only upon further examination that one discovers each one of the murals has been created in order to record a traumatic event experienced in a recent past. The commu- nity's artistic expression is indeed a representation of the layering of histories and memories and it uses its architecture (or architectural fabric) as the canvas.

In order to better understand 'La Comuna 13 ', its people and its artistic expressions, we must first go back and review the events which moulded it. In 1616, Medellin was founded by Spanish conquistadores in the valley of Aburra and as further explained by Drummond et al. in the nineteenth century, the city began to grow due to both the production of gold and coffee. Violence began to spread during the 1950s due to differences amongst leftist revolutionary groups and their battle for territory in the countryside. As a result, hundreds of thousands of poor Colombian farmers and their families fled their rural environment for the cities. In Medellín alone, the population swelled from 358,000 in 1951 to $1,071,000$ by 1973 . Not surprisingly, most settled in the rapidly expanding slums built on Medellín's mountainsides in the northern and western parts of the city - ; La Comuna 13 serving as a prime example. In their article, Drummond et al. explain how during this time, it was part of the MMP (Medellin Master Plan) to regulate these growing settlements of migrants on the steep slopes. MMP leaders struggled to deal with these neighbourhoods and were often unable to provide them with basic services such as sewage, water, schools, roads and health care (Drummond et al., 2012). The 1980s saw a rise in terrorism and criminal activity throughout the country. This in turn gave rise to violent paramilitary and self-defence groups who practiced their own brand of vigilante justice, at times with the tacit approval of the Colombian military. Of all Colombian cities, Medellín experienced the most acute violence and bloodshed. At the end of the 1990s, a process of social cleansing began, largely supported by paramilitary groups backed by the state, especially during the two terms of President Álvaro Uribe from 2002 to 2010. Patrick Naef outlines how the main objective was to eliminate guerrilla presence in the city, but the social cleansing also targeted other individuals, such as prostitutes, union leaders and petty criminals. La Comuna 13 became a particularly infamous symbol of this process, after several military and paramilitary operations took place there. The operations Mariscal and Orion in 2002 are certainly the best known and were the most traumatic for the local population (Naef, 2018).

For many residents of Medellin, the comunas generally still represent a significant link to violence and symbolise a city characterised by crime and drugs. Over the years, a new reputation has begun to emerge. One which is linked with art, culture and turning this community's turbulent past into some- 


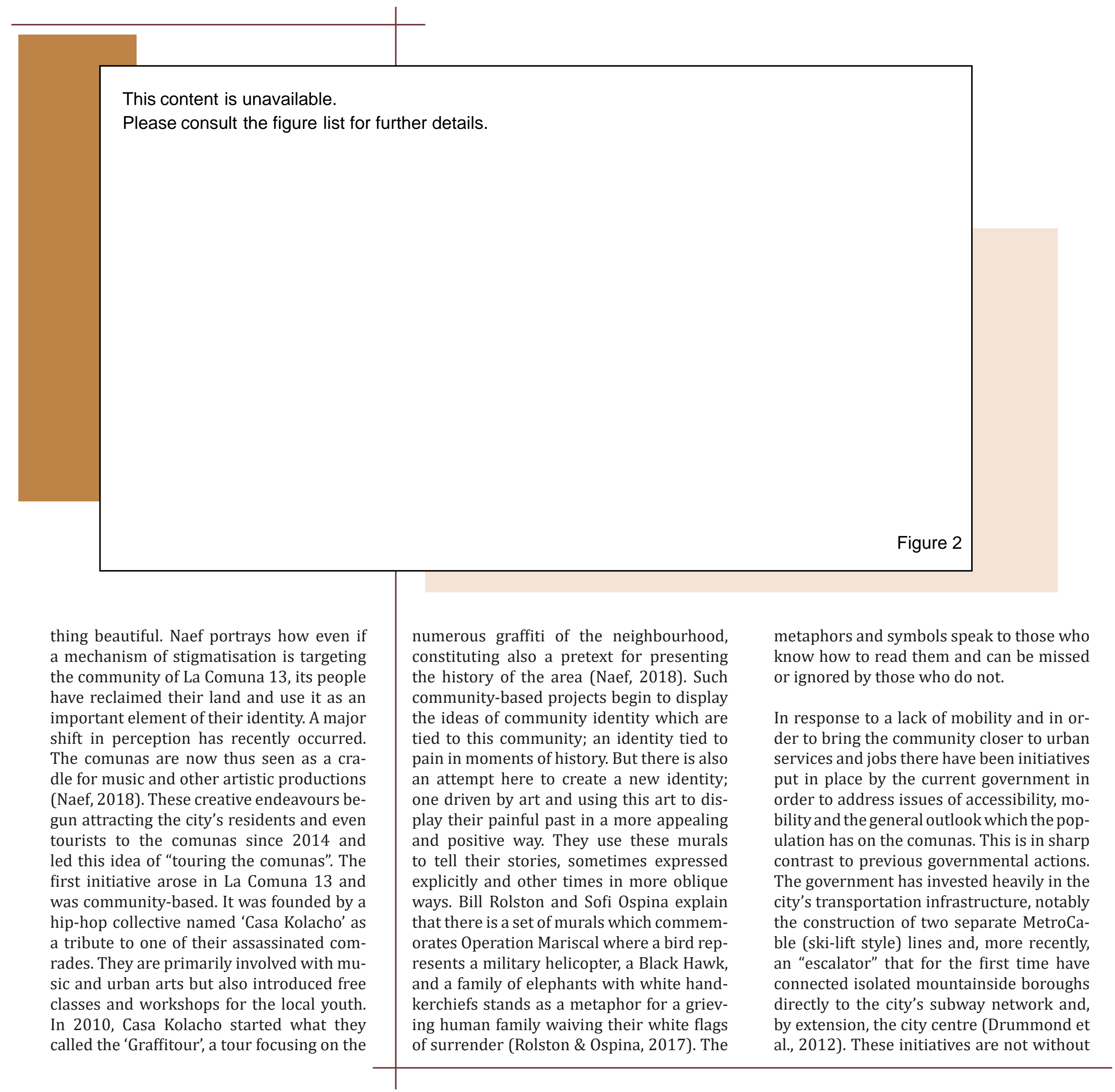


This content in unavailable.

Please consult the figure list for further information.

Figure 3

flaws and complications. For example, the MetroCable has a limit of 3,000 passengers an hour which reduces its functionality as a mass-transit system. Adding to this issue is the organisation of the neighbourhood which means that some passengers endure long walks in order to reach their nearest station and then having to sometimes wait thirty minutes or more in order to board. Something else which may be of further detriment is the fact that 'bulky personal effects' are not permitted (Drummond et al., 2012). For some residents of La Comuna 13 , their source of income is travelling into the city centre to set up stalls and sell on the street. Not being able to carry what they need in order to do that on the MetroCable hinders their ability to earn money as this is one of their main transports to the city centre.

A survey carried out by Drummond et al. offers great insight into how the resident of La Comuna 13 view their community. It revealed that $68 \%$ of resident acknowledge that they would like to move out of the Comuna (Drummond et al., 2012). This reinforces the necessity to re-examine the living conditions of this community. As mentioned above access to the city centre is something which the government has tried to address but a more radical solution may be needed, such as enabling the community to move into the city centre. Judging by the survey results, members of La Comuna 13 value knowledge, economic growth, community engagement and artistic expression. This design-led research aims to provide the community with an architecture which may not only accommodate all of these aspirations but promote them.

With the understanding of La Comuna 13's turbulent past and its origins, it becomes evident that pain and moments in history as well as stories are a key part of this community's identity. 'Place identity' can be defined as 'set of meanings associated with any particular cultural landscape that any particular person or group of people draws on the construction of their own personal or social identities'. Following these ideas, Ashworth et al. consider the transformation of space into place 'through traditions, memories, myths and narratives and its uniqueness confirmed and legitimated in terms of their relationship to particular representations of the past' (Ashworth et al., 2007). This becomes very relevant when considering how La Comuna 13 and other such communities identities' have been created around memory and history (their memories and histories). The people have found a way to express this identity through urban art in order to perhaps not only perhaps talk to themselves but also introduce themselves to outsiders. This combination of artistic expression and art have turned this community's 'space' into a place. This begins to highlight ideas such as memory, place-identity, layering and the relationship between art and architecture. These ideas will be further explored in the following chapter as they will become the key themes for the research.
This content in unavailable.

Please consult the figure list for further information.

This content in unavailable.

Please consult the figure list for further information.

This content in unavailable.

Please consult the figure list for further information. 


\section{"La Comuna 13 hoy es visible y eso permite que se entere el mundo que aqui pasan otras cosas y que La Comuna 13 no es esa historia violenta que vivímos" - Kbala*}

"La Comuna 13 is visible today and that allows the world to know that other things happen here and that La Comuna 13 is not that violent history that we lived" 


\section{CHAPTER 2: THE IDEAS}

Capítulo 2: Las Ideas 


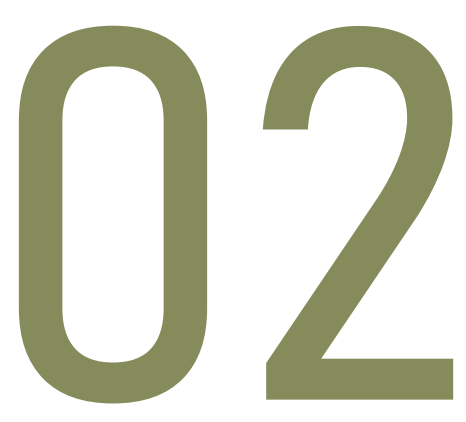




\section{La Memoria}

A significant theme within the expression of La Comuna 13 is memory. The visual recording of their painful memories and experiences through art help form identity. Their architecture does not necessarily embody these memories but simply carries them, as canvases. Eleni Bastea states that architecture can capture fleeting or insistent memories into tangible and buildable forms. Yet memory creates a special relationship with space, holding onto the essence of it, the best and the worst (Bastéa, 2004, p. 1). This holds true for La Comuna 13 as their current community captures memories of war, bloodshed, and pain. Paul Goldberger explains how we all have our own memories of buildings and places, of the architecture in which we have lived. It is these memories which set a tone the way that we experience (goldberger, 2009, p. 140). Kent C. Bloomer and Charles W. Moore, support this idea as they describe how we do develop memories of an inside world which include a panorama of experiences from the environment and etched into the "feeling" of our identity over a lifetime of encounter with the world (Bloomer, 1977, p. 49). This is important to consider in the process of designing for this community as there is potential for their experiences in their current environment to determine how they respond. Therefore memory becomes relevant to the design process as it could entirely influence integration of the community into the new.

Considering these ideas, Sabine Bitter and Helmut Weber argue that while architecture can hold intrinsic meanings and memory, it can also be reworked to project with a vastly different historical narrative than the one it originally represented (Bitter \& Weber, 2018, p. 39). This is a particularly important concept for this research as it indicates that even though architecture can

This content is unavailable.

Please consult the figure list for further details.

Figure 7

hold memories, it is still possible to re-work this architecture to embody a whole new meaning. The building itself will have memories which it carries and there will need to be some negotiation with how strongly these memories surface in the new design. Then how the memories of the new occupants will influence their experience of this new space.

The concept of memory will be an influential theme throughout the design process as it forms such a core part of this community's identity and experience of architecture. As "etched into their hardened fabrics of brick and stone, records of human interaction mark cities as sites of endurance as well as of change" (Bastéa, 2004, p. 25).

In the specific case of La Comuna 13, the memories are manifested through murals on external walls. These form the direct experiences of streets, passages, squares within the precinct of the community. As a result, the inhabitants experience their communal urban spaces in a continued reference to their collective past and formed identity. It is not the architecture within that holds the community's memory but its skin (the external wall), that serves as a canvas and its layers, to form the public realm.

This content in unavailable.

Please consult the figure list for further information. 


\section{PLACE-IDENTITY}

Identidad de lugar

Identity or place-identity are concepts which are closely related to the ideas of memory, community, and a sense of belonging which are clearly emerging in La Comuna 13. They also become key themes to consider in the process of design to ensure that this community can develop a sense of belonging and attachment to the interventions being designed.

VHarold M. Proshansky et al. describe 'Place-identity' as "a sub-structure of the self-identity of the person consisting of, broadly conceived, cognitions about the physical world in which the individual lives". Included in said cognitions are things such as memories, ideas, feelings, attitudes and the experiences related to our day-to-day physical environments (Proshansky et al., 1983, p. 77). With this idea of place-identity, there is also the idea of place attachment. Setha M. Low and Irwin Altman explain that the word "attachment" emphasises affect. While the word "place" focuses on the environmental settings to which people are emotionally or culturally attached. Place refers to a space which has been given meaning through personal, group or cultural processes (Altman \& Low, 1992, p. 5). Both Place-identity and Place Attachment are ideas which apply to La Comuna 13. This is a community which has developed a sense of belonging to their current surroundings through their shared memories but they have also built a cultural attachment through their art. This idea of creativity is related to place attachment by Altman and Low as they illustrate how the bonding of people may be to the environmental setting themselves and this may be because they permit control and creativity, and they provide opportunities for personal displays (Altman \& Low, 1992, p. 7). Their current physical setting, allows the people in La Comuna 13 to express themselves freely through this new culture of artistic expression which they have developed in order to identify themselves.

This affects the design of the interventions and how these would affect their sense of belonging and Place-identity. Proshansky et al. state that it is possible that when groups are relocated, they often feel 'lost' in their new setting and long for the old home and neighbourhood. Depending on how severe the discrepancy between the old and the new locations, there is a danger that a sense of belonginess to the new physical setting may not be achieved (Proshansky et al., 1983, p. 79). This issue is significant to this research as the aim is to create spaces which this community can identify with and develop a sense of belonging to.

It is important to consider the factors which create a sense of belonging for this community and to incorporate these into the design to continue allowing for the creativity, the memories and the community aspects to take place. Sometimes the occupants can take charge of reducing or eliminating the discrepancies between their old physical setting and the new. They can do this by identifying what is 'right' and 'wrong' with the physical setting and what has to be done to bring about change in it to reduce the discrepancy between it and the place-identity associated with the other place (Proshansky et al., 1983, p. 80). If the new architecture is one which facilitates the continuation of the creation of art and the community activities such as art and dance classes then the occupants may take care of covering the new walls with street art and embracing the new allowed space for cultural activities.

Frashid Moussavi presents another aspect of identity not in relation to the human ex- perience but to materiality. It becomes interesting to begin looking at how materiality can contribute to this idea of identity. Moussavi talks about how new meanings and ideas can be generated through recycling, repositioning, and the reorientation of existing ideas. And rather than attaching an artificial symbolism to a project, we use hybridisation as a tactic of introducing contamination into already existing material (Moussavi, 2007, p. 83). Seeing as this community is currently accustomed to painting on brick walls, it will be important to provide this materiality in the design for them to have a sense of familiarity. But hopefully, in a new setting, new meanings and ideas will come even if the material stays the same. 


\section{ART + ARCHITECTURE}

Arte + Arquitectura

The relationship between art and architecture is one which has been evident throughout history. Both practices have informed and influenced each other in almost every aspect, making it an intricate relationship. Many would argue that one cannot exist without the other as they display overlaps in physical, abstract, and literal ways. This is true at many different scales of influence. In the simplest fashion, an architectural environment can influence the process by which art is produced. As described by Elizabeth Vente, things such as qualities of light, flexibility of environment, and the tectonic and material natures of a building can be influential in the process (Vetne, 2009 , p. 1). This becomes relevant to this research where architecture is seen as the canvas, and the materiality of this canvas becomes authoritative of the art which is to be created on it. Vetne also discusses how in modern times, many of the purposes of coexistence between art and architecture are centred around placemaking strategies within the urban fabric, an example of this being street art (Vetne, 2009, p. 7). Malcom Miles reinforces this idea as he states that art activities provide a community with a focus and increase its sense of identity. It creates an increased awareness of the communities needs and determination to create change (Miles, 1997, p. 65). There are also notions that the addition of street art onto architecture can help to make a place more interesting and attractive, and also to highlight the identity of different parts of not only the community but of the building as well (Miles, 1997, p. 66).

So then with this new idea of the building becoming a significant part of the equation, 'the wall' becomes an integral part of the relationship between street art and architecture. Andrea Mubi Brighenti examines the idea of walls as being built as part of a strategy aimed at controlling people and their activities by controlling space. Walls introduce boundaries into a formerly smooth space, they enable the separation of a 'within' and a 'beyond'. Most importantly, they determine the possibilities and impossibilities of encounters (Mubi Brighenti, 2010, p. 322 ). In this sense, it would be interesting to consider that the encounters being referred to, are the encounters between street artist and wall, and the public and the wall. Andrea explains how the wall offers a visible surface, which becomes a surface of inscription for overlapping traces (Mubi Brighenti, 2010, p. 323). These traces being left behind by the artists as records of their histories and memories, particularly in La Comuna 13. It is these memories and histories which have created this sense of connection and community among its inhabitants. Dovey et al. argue that the graffiti/street art covered walls become connections not just between the artists but also between different parts of these communities and between these communities and the broader world (Dovey et al., 2012, p. 38). This idea is profoundly applicable to La Comuna 13 as the art which they are producing is becoming the main reason they are known and acknowledged by the wider city and even globally. Keeping this in mind, it could be argued that the abundance of street art could be linked not only to a supply of blank walls and artistic talent, but also to the demand for street art (Dovey et al., 2012, p. 38).
The link between architecture and street art is, in essence, quite simple. It is the relationship between the wall as the canvas and the art as another layer which is added to the architecture. This layering not only adds value to the architecture, but it also forms a key aspect of the community's identity. It will also be important to incorporate some of the ideas outlined above such as the importance of materiality in order to facilitate art and providing a blank canvas for the artists. This will hopefully enable an ease of developing a sense of belonging. 


\title{
LAYERING
}

\author{
Capas
}

The simple act of painting a piece of street art on a wall evokes the idea of layering. To further this, there is then the layering of forms and colours which takes place to make up the finished piece. Tracey Gray proposes that there are three categories which encompass this idea of 'Layering': Literal, Phenomenal, Metaphorical (Gray, 1991, p. 7). In order to simplify the idea, this analysis will follow the same three categories as there are various authors who have ideas which contribute to and reinforce each category.

Gray describes Literal Layering as the layering which is immediately sensed by the viewer. The layers are simultaneously perceivable within the same sensual field whether it be visual or tactile. This is real existential layering (Gray, 1991, p. 8). This idea of Literal Layering is highly evident in art techniques such as the Cubist form of painting. In Cubism, objects are divided into pieces and segments which are then put back together to create an entirely new perspective of the object. An example of this is the painting by Georges Braque titled 'Bottle and Fishes' (Figure 9). This is a painting of bottles and fish which are sitting on a plate, on top of a table. The objects are reconstructed through the layering of shapes to look nothing like we would normally see. The act of layering is highly evident as you can see how shapes and forms are painted over top of each other to create the overall image, much like graffiti or street art. Looking at the way this is done with Cubism, this research would argue that there is a way to reverse the process. Take the piece of street art, break that down into just shapes/forms and use these shapes to design the interi-
This content is unavailable. Please consult the figure list for further details.

Figure 9

or architecture in terms of spaces and how these relate to each other. These relationships between forms would then start to create ideas of layering within the architecture. Michael Davis explains that such layering is a type of three dimensional collage: instead of placing pictures or planes on one another it is possible to overlap spaces into one another (Davis, 1984, p. 45). In plan, these spaces would look like planes which have been placed on one another, but the spaces would simply be overlapping (Figure 10). Davis also explains how changes in level, use of colour, texture, lighting, and transparent walls are all means of creating layering and defining spaces yet one of the most effective techniques is to rely on simple geometric shapes (Davis, 1984, p. 46).

Phenomenal Layering is described by Tracey Gray as not being evident from the first reading, a second, third or even fourth reading may be required to seek out the qualities of layering, a mental completion of the form is required (Gray, 1991, p. 47). In this type of layering, the layers may not be as clear as literal layering or it may just take further interpretation to ascertain where the layering is occurring. Gray further explains that the superimposition of elements allows only parts to be seen, elements obscure the other elements instead of allowing them to be seen as with literal layering. This leads to the excitement and mystery as one walks through finding a succession of elements partly hidden, partly revealed (Davis, 1984, p. 56). This begins to obscure the idea of layering where parts of space or an interior may be revealed just enough to peak the curiosity of the users in order for them to continue moving through the spaces. An example of phenomenal layering is The Schroder House as the layers created in the architecture need to be found but once they are, the viewer can see them simultaneously. This house is seen as both a whole but also as a sum of all its individual parts (depicted in following page). This then introduces a new possibility for interior architecture as screens and walls can be used to partially reveal the layers within the space. Following 


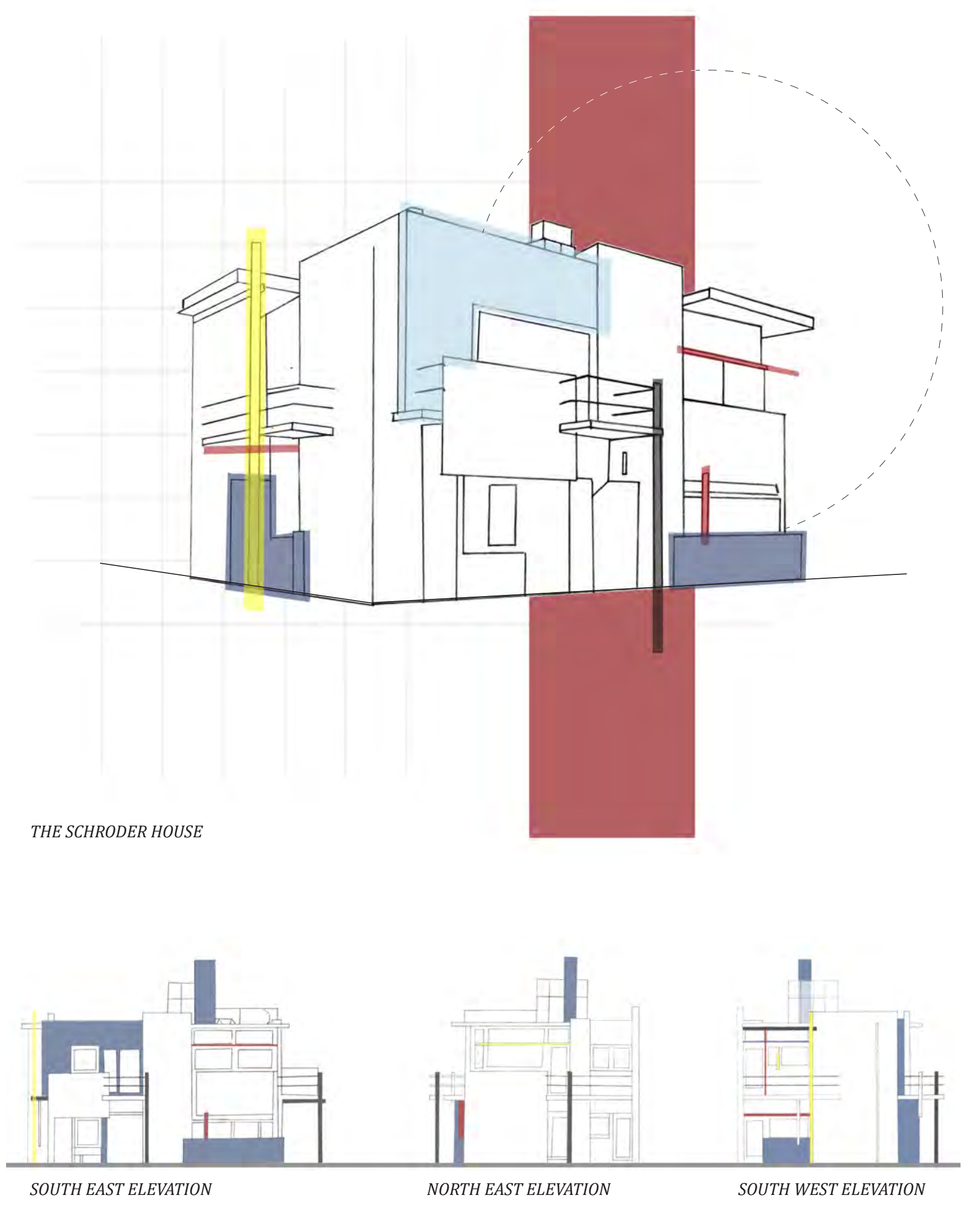


this idea, Davis discussed how the boundaries between spaces may become so blurred that the spaces flow into one another. What 'walls' there are define both a space as well as a continuity (Davis, 1984, p. 59).

Lastly, Gray defines Metaphorical layering as embodying the layering of ideas, meaning and symbolism. Buildings are designed with inherent conceptual and symbolic themes and in addition to these ideas further meanings are bestowed upon the building by others (Gray, 1991, p. 85). This is potentially the most significant category of layering when it comes to this research. This community adds another layer onto the architecture with their street art and this, therefore, adds meaning to the architecture. It must be noted that Metaphorical layering is not something which can take place during the design phase of the project as it is more something which is bestowed upon the buildings by others. This idea reinforces the intention of this research and the ideas previously discussed as it validates the importance of facilitating the creation of art for this community within the new interventions.

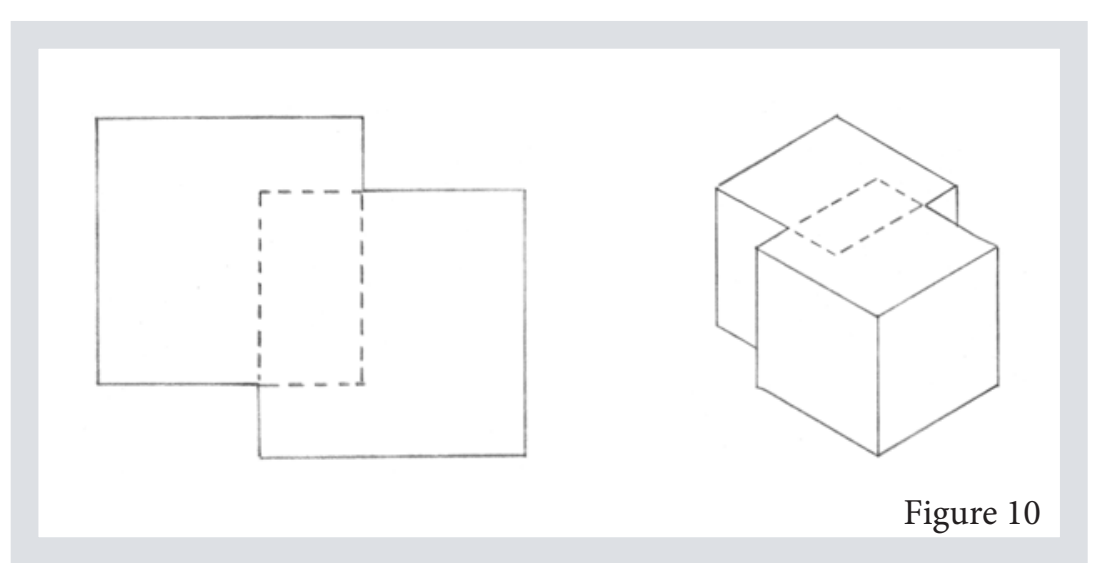




\section{CHAPTER 3: THE WALL}

Capítulo 4: La Pared 


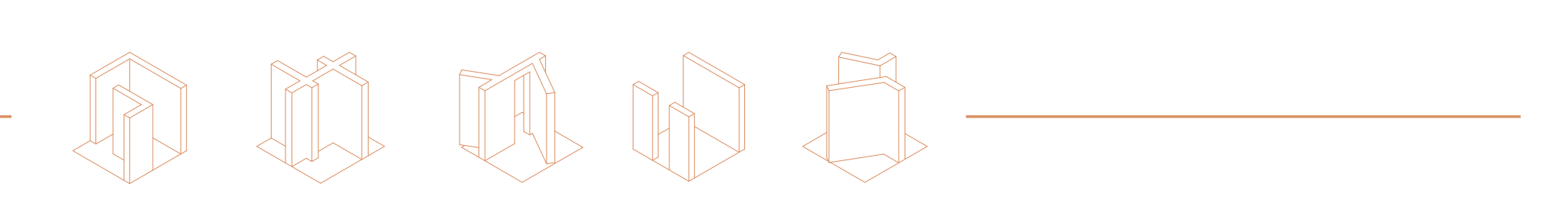




\section{THE WALL}

La pared

Due to the nature of this work and the acknowledgement that it is essentially an exploration of the significance and potential use of walls in different programmes, 'The Wall' then becomes a significant aspect of the research and the design process. It becomes important to understand the effects of the wall as an architectural element on people and space. The ideas of public and private space as well as public and private walls also need to be understood for this design-led research.

It is necessary to recognise that the arrangement and ordering of forms ad spaces can also determine how architecture might promote endeavours, elicit responses, and communicate meaning (Ching \& Ching, 2014, p. 4). Timothy Horne explains how space is created by boundaries by encompassing architectural elements of form. Our experiences of space are determined by our understanding of the physical system and organisation, our perceptual recognition of the physical elements and our conceptual comprehension of the relationships within the physical organisation of a building (Horne, 1989, p. 1). Following these ideas, it becomes evident that the existence of a physical work of architecture relies on a logical use of the basic element of architectural form such as walls, floors, roofs and etc. Of these architectural elements, the wall is the most active as it regularly interrupts our immediate visual field and influences our movements within and throughout a space. "Walls are an architectural event, the junction of exterior and interior, the enclosure of space, or a simple boundary." (Horne, 1989, p. 1).
To begin this exploration, 'the wall' can be broken down into two elements: the line and the plane. Frederick Jules attempts to simplify the relationships between these two elements by saying that a volume usually contains an activity, while a plane separates activities and a line directs the user to said activity (Jules, 1979, p. 42). And although the basic logic here may be correct, the relationship between elements requires further analysis. Frank Ching illustrates how a line, in describing the path of a point in motion, is capable of visually expressing direction, movement, and growth (Ching \& Ching, 2014 , p. 8). The line created at the edge of walls serves as guidance for the user of the space by depicting the direction of the wall and the size. The line becomes a significant tool for the users in terms of understanding and navigating space. These lines are directly linked to the planes which form the wall. Ching explains how in the composition of a visual construction the plane serves to define the limits or boundaries of a volume. If architecture as a visual art deals specifically with the formation of three-dimensional volumes of mass and space, then the plane should be regarded as a key element in the vocabulary of architectural design (Ching \& Ching, 2014, p. 18). As a planar element, the wall inherits many qualities and possibilities. Looking at a plane alone, it has a frontal quality. But due to the nature of the wall, it derives a front and a back, increasing the possibilities of what can happen on the planes which make up its structure. These two faces or planes can be similar or they could be entirely different in order to contribute to different spatial qualities. Timothy Horned describes how the built wall will be articulated by the proportion, opacity, transparency, scale, definition, colour, texture, pattern, configuration, shape and edges (Horne, 1989, p. 3). All of these factors contribute to making the wall an active architectural element, consisting of many other elements which contribute to its aesthetic and functional abilities.

After considering the wall on its own, it then becomes important to begin thinking about the multiple walls which normally make up a space or volume. Ching explains how vertical forms have a greater presence in our visual filed than horizontal planes and are therefore more instrumental in defining a volume of space and providing a sense of enclosure and privacy for those within it (Ching \& Ching, 2014, p. 134). They also serve as a means of separation between

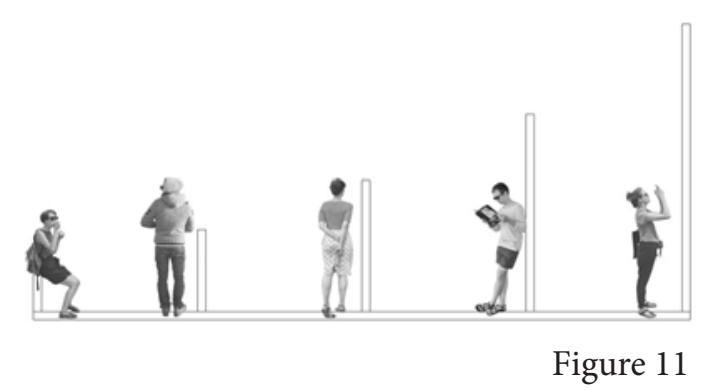

spaces and establish a boundary between inside and outside.

Following this idea, the wall can be used to define the volume of a space. An example of this would be a freestanding element within a space which divides the volume into two separate yet still related areas which can be seen in Figure 12. This means that the wall can be used to cut the continuous filed of the architectural volume and help to create an 
open plan of spaces that merge with one another. There is also the simple approach of having two vertical planes parallel to each other which automatically create a volume between them as seen in Figure 12. If one of these planes changes and becomes differentiated from the other through either a change of form, colour or even texture, then a secondary axis , perpendicular to the flow of the space, will be established within the space (Ching \& Ching, 2014, p. 154). An experimentation of the changing of walls was carried out in order to further understand these ideas, which can be seen on pages 3132.

While considering multiple walls joining in order to create a volume, it becomes meaningful to also consider the angles at these intersections. Frederick Jules discusses this
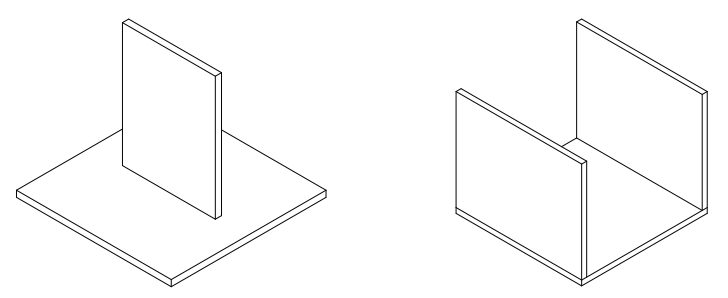

Figure 12

in relation to Gestalt Implications and states that for the various angle sizes formed by the intersection of lines, planes or even volumes: an acute angle implies direction toward the apex of the angle; a 90-degree intersection implies discontinuation of the surfaces at the apex and less direction than acute or obtuse angles; an obtuse angle or curve implies continuation along the surface forming the angle. Additionally, they all imply closure; the more obtuse the angle, the softer the sense of closure (Jules, 1979, p. 46). This changing in angles means that there is a dynamic quality to the walls and therefore the architecture. They are a manifestation of the architects' intent for how people will move through the space or perceive the space. Robert Venturi states that the essential purpose of the interiors of buildings is to enclose rather than direct space and to separate the inside from the outside (Venturi, 1977, p. 72). However, there is potential for the purposeful use of the vertical plane or wall to allow interior spaces to direct and guide the users; to create a spatial experience of moving through an architecture while being guided by the architecture itself. 
There must also be consideration into the relationship between the vertical plane and the horizontal plane. T. Horne explains that we occupy and move within the horizontal plane, it is our base for all human perception and experience. We can then engage with the world through a physical interaction with the vertical direction. This vertical direction penetrates the horizontal plane which creates visual and spatial tension. The initial perception of any architectural elements is firstly two dimensional; we see the horizontality and the verticality (Horne, 1989, p. 9). The relationship between horizontal and vertical, ground and floor, becomes evident. It is a matter of understanding whether the walls are emerging from the ground or floating above it. It is about if the connection becomes an edge or a gap. There are various ways in which this relationship can be articulated as can be seen on pages 29-30.

Taking all of this into consideration, moving forward into the design becomes a much more complex process. It is not just a matter of arranging walls in a certain way with certain parameters in mind. It becomes a careful exercise of considering the affordances of these walls and the navigation of the users. The wall becomes a powerful tool in terms of not only creating the interior architecture but also in manifesting an idea.
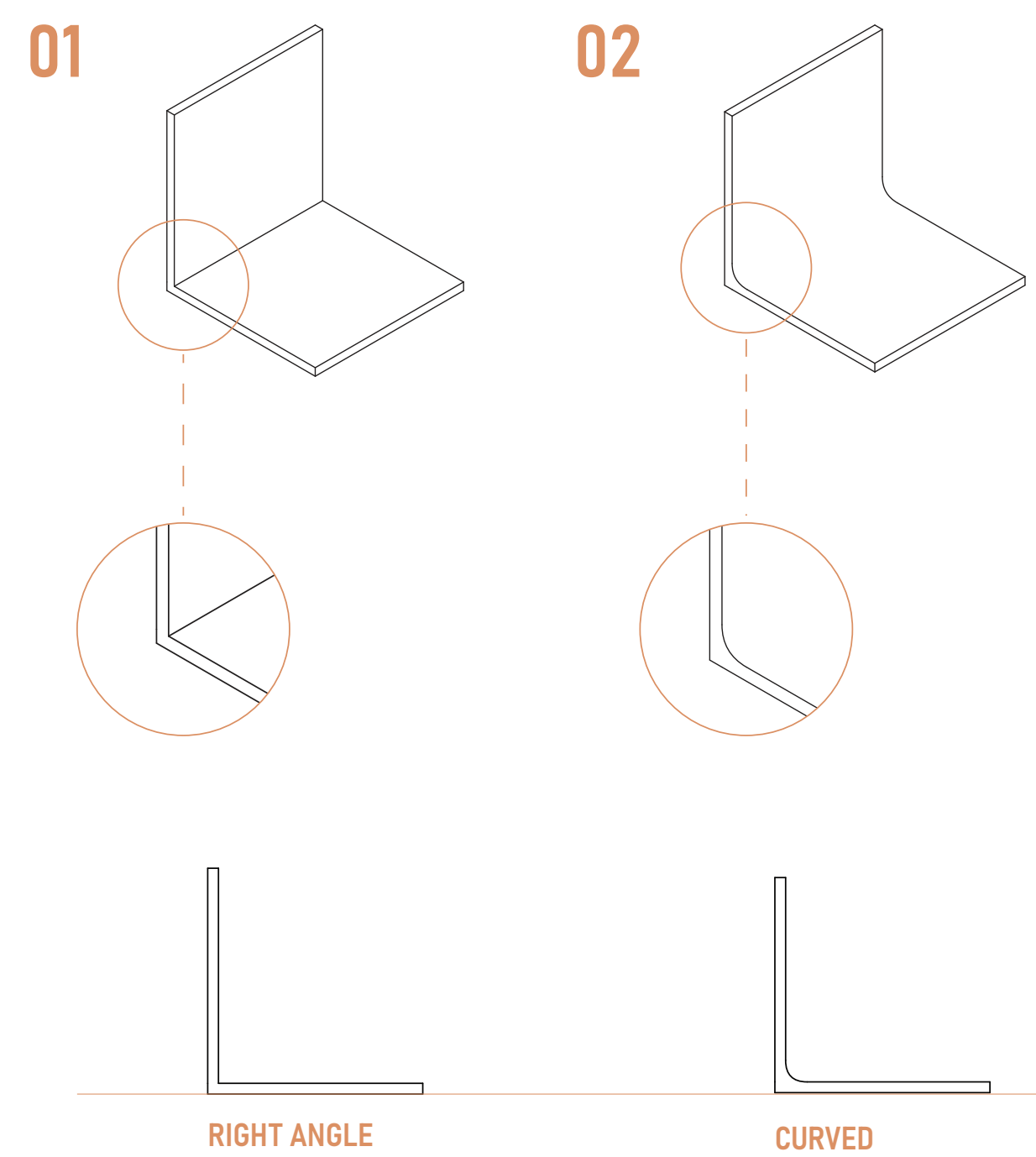
03
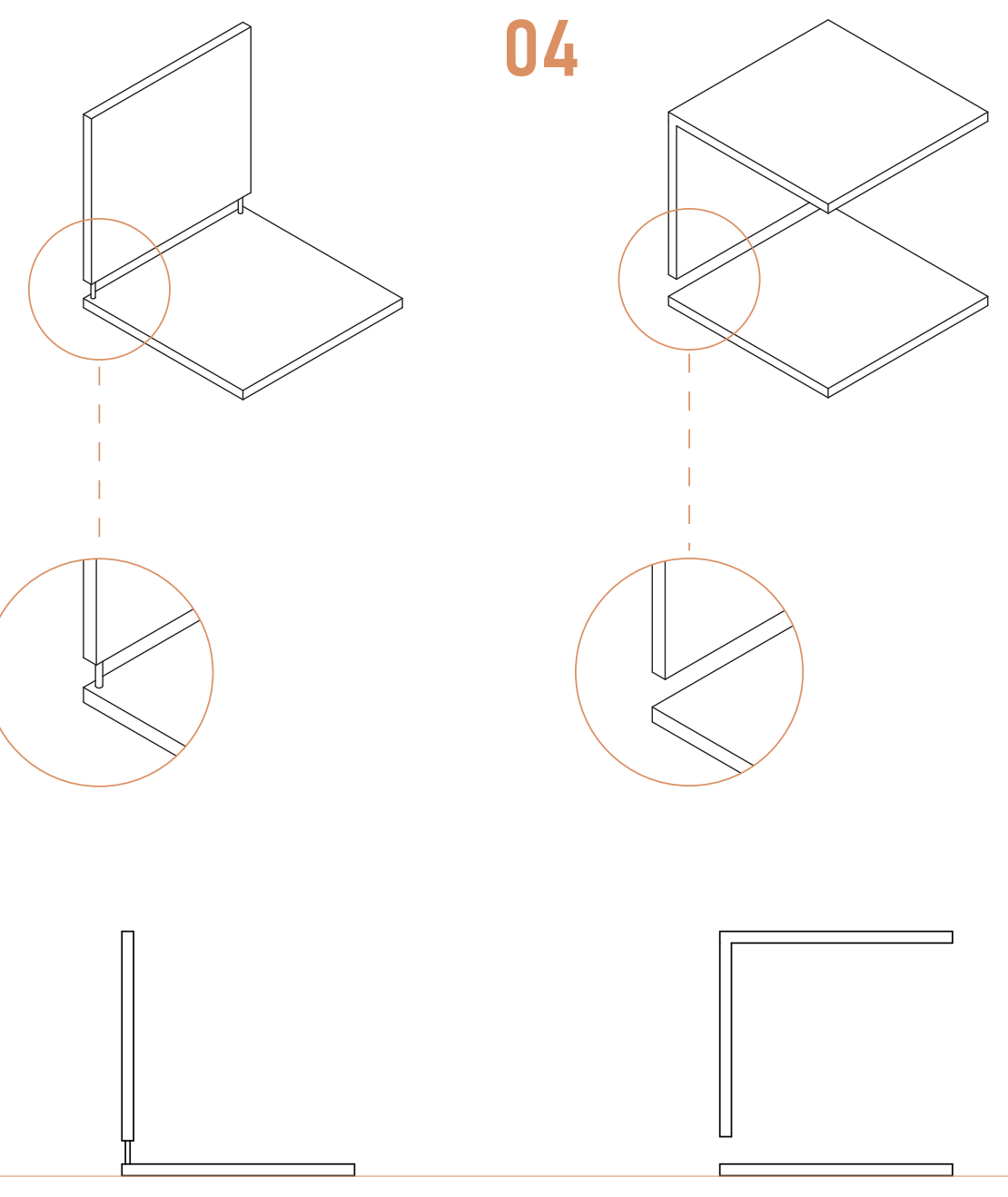

GAP WITH BAR
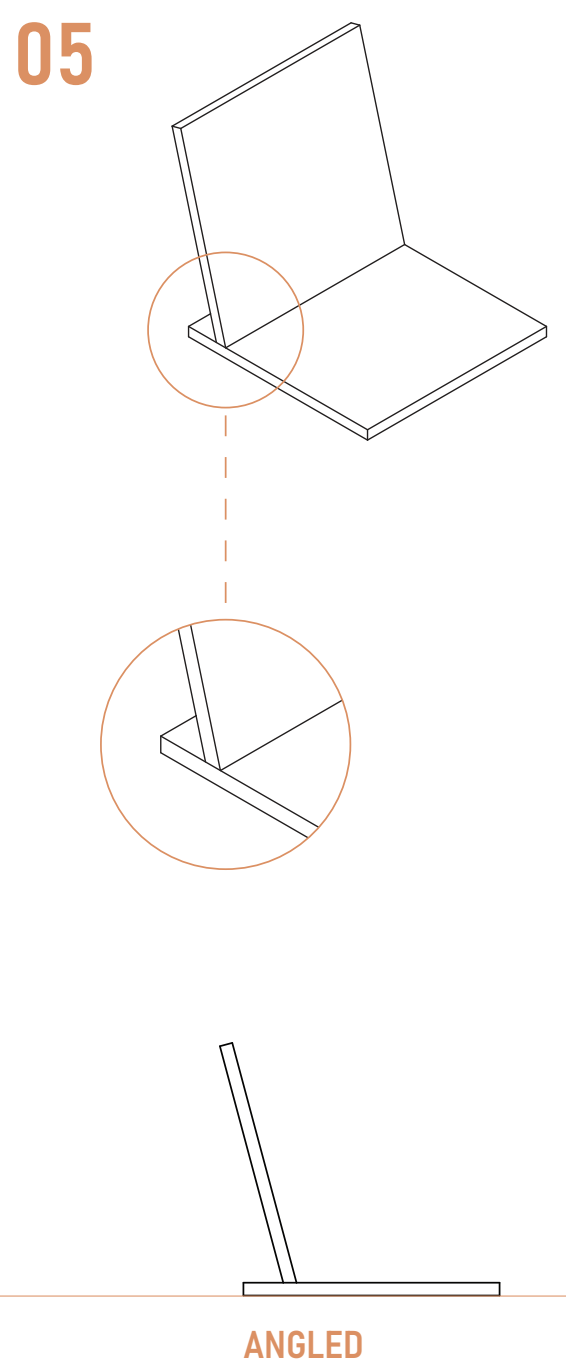

06
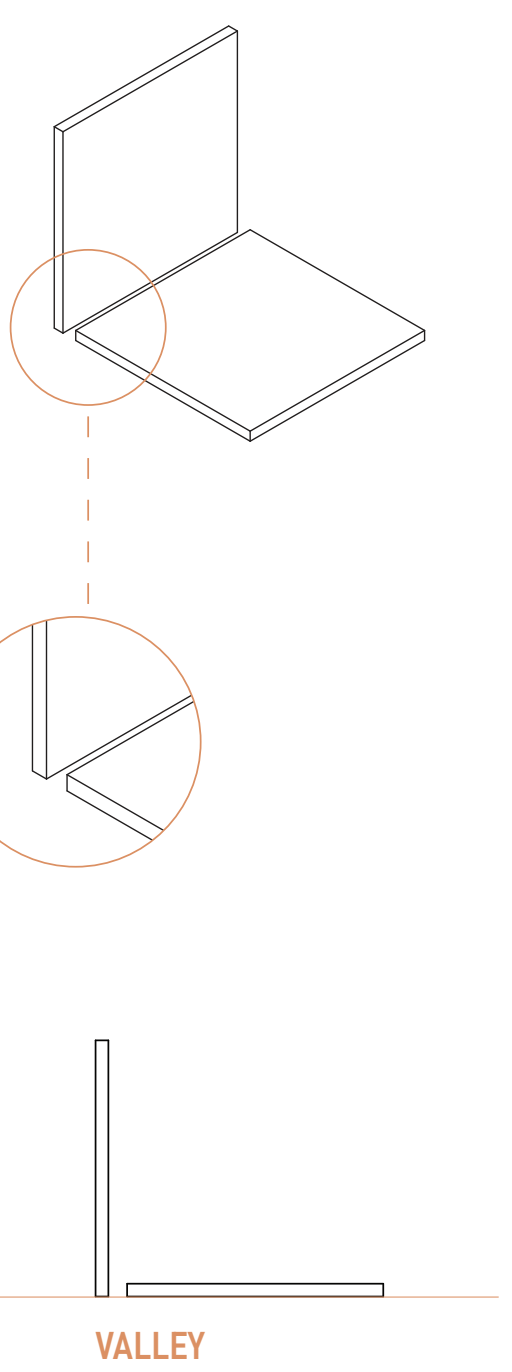

30 
02
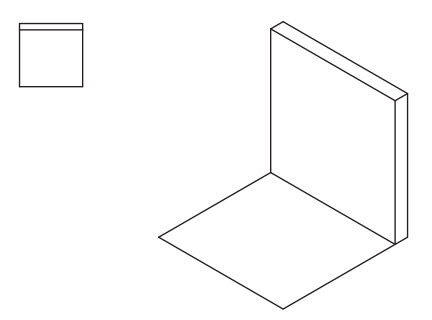

ㅁ.

$\square$

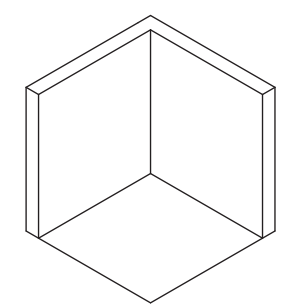

$\square$

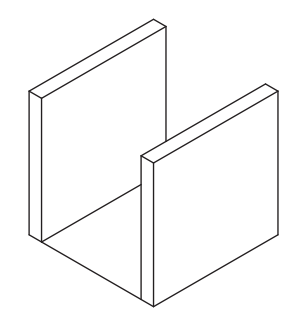

$\square$

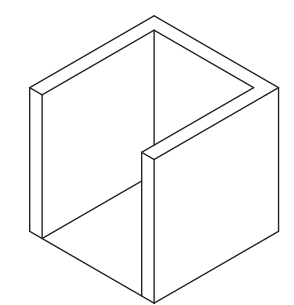

$\square$

$\square$

E

$\square$

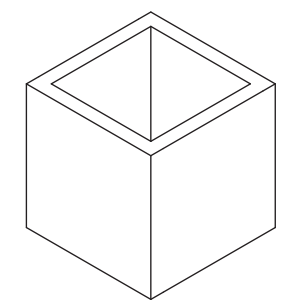

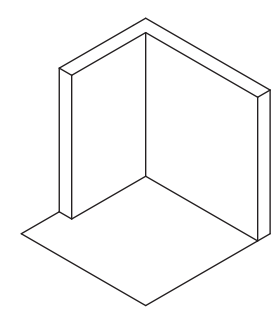
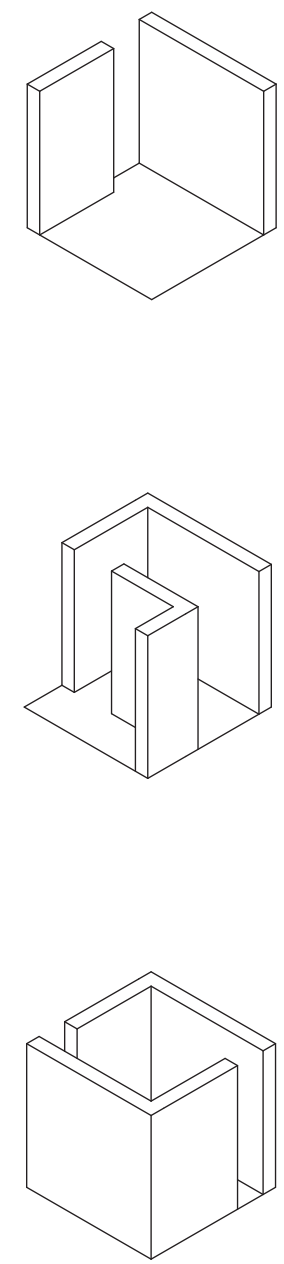

1
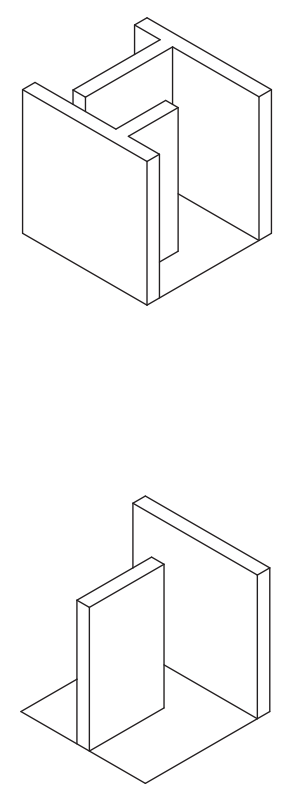

E

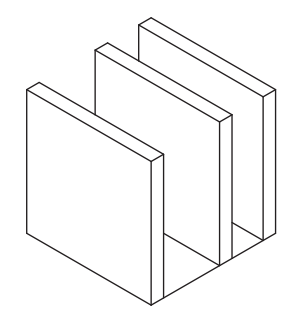

$\square$

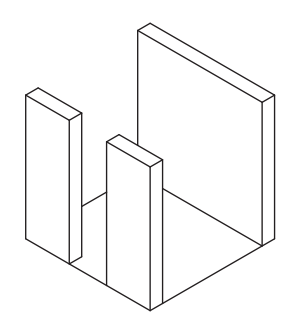

$\square$

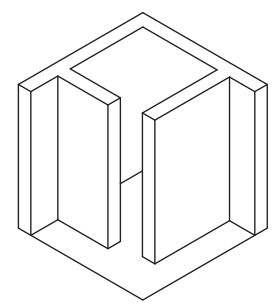

03

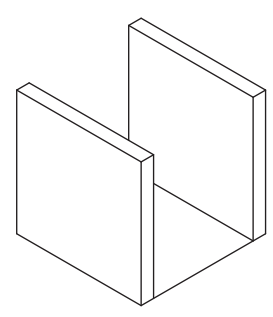

田

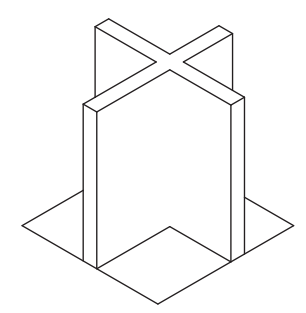

$\underline{\square-1}$

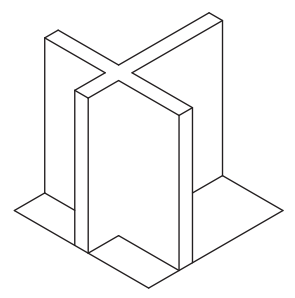

파맘

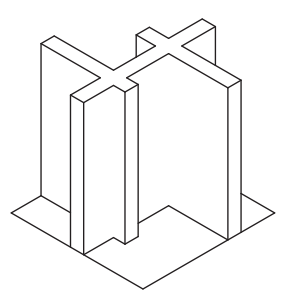

투

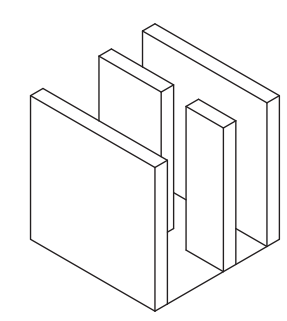

$\Rightarrow$

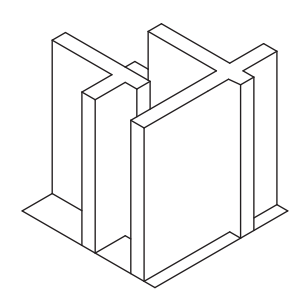

四
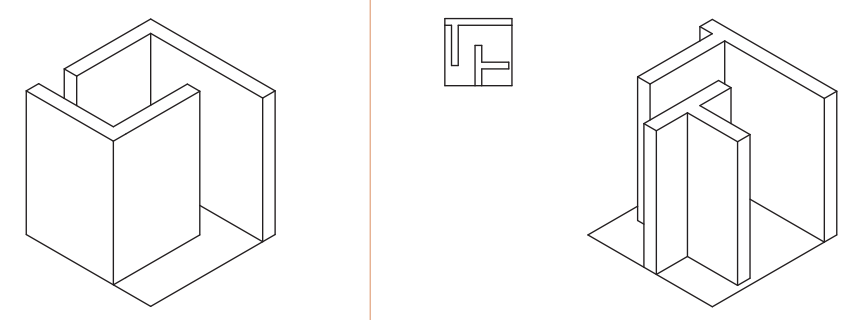

톨

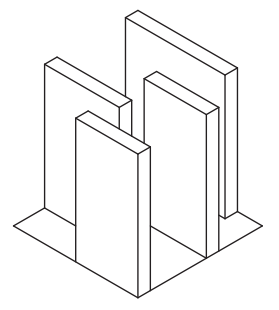

谣

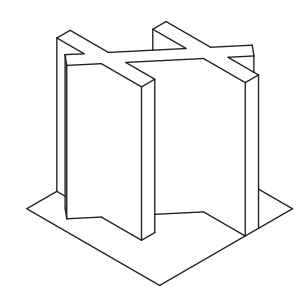


08

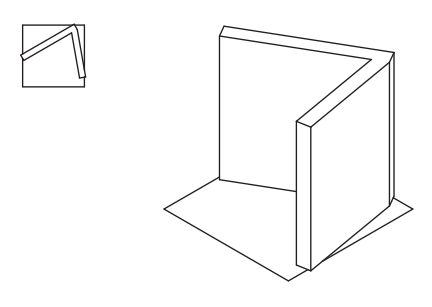

N

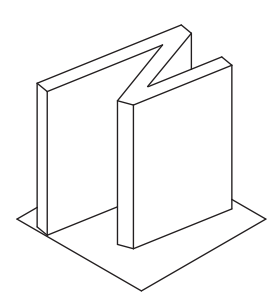

$\mathbb{N}$

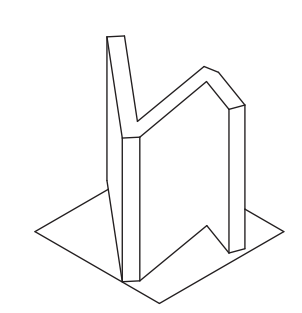

圆

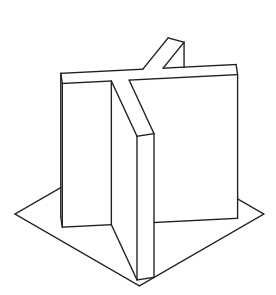

逄

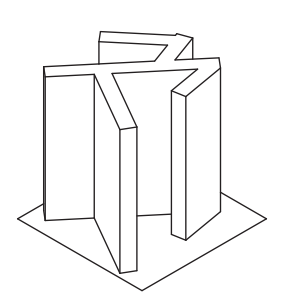

E

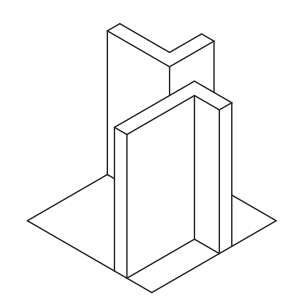

E

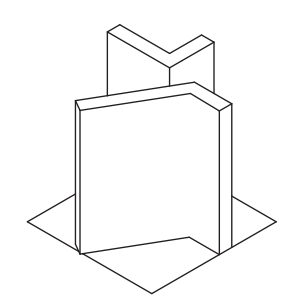

䧄

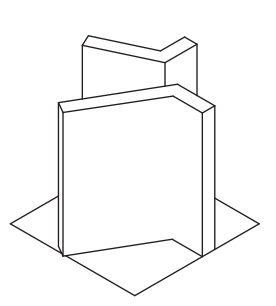

因

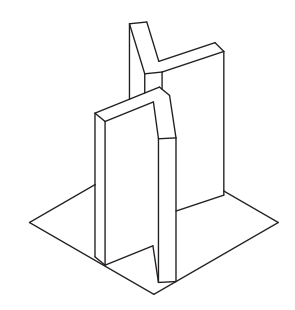

E

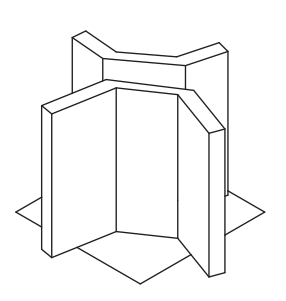

09

10

吜

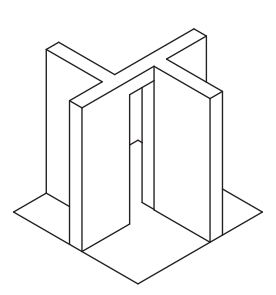

b

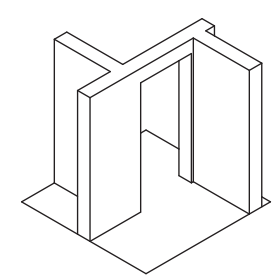

相

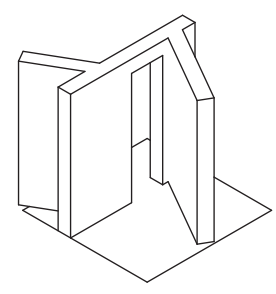

$\sqrt{ }$

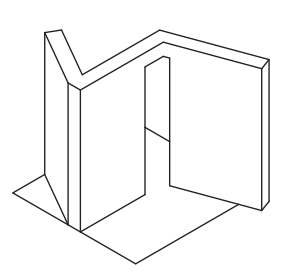

$\sqrt{5}$

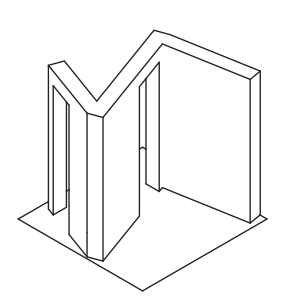

!

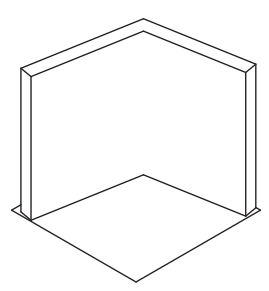

『

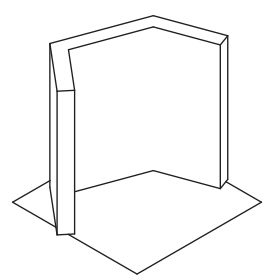

(1)

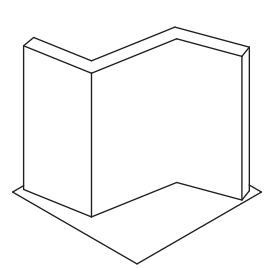

2

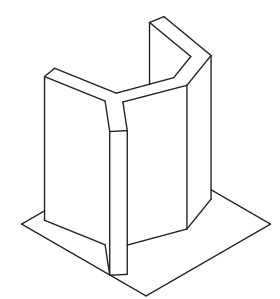

EI

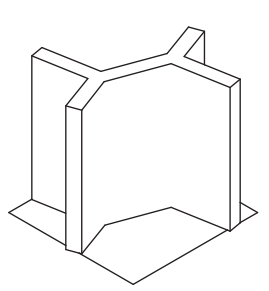

圆

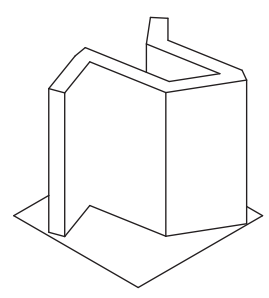

今

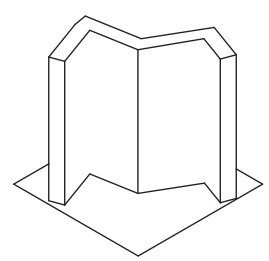

D

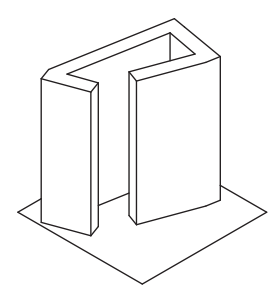

四

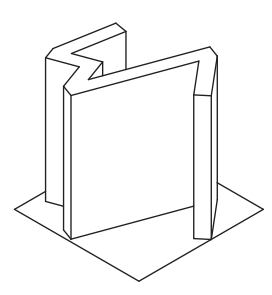

因

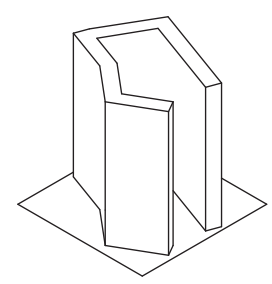




\section{CHAPTER 4: THE ART}

Capítulo 4: El Arte 
04 


\section{THE ART OF LA COMUNA 13}

El arte de La Comuna 13

The previous chapters briefly exposed the story behind in the murals of La Comuna 13 and some of the elements and ideas displayed through them. The story behind the murals as well as the wider community's increasing interest in them is to be noted. As writer N. Mackenzie explains, local artists started to paint the murals depicting the violence that had occurred in the community and its aftermath.; following this the local government embraced the opportunity to encourage more work by financially rewarding artists for their creations. As a result, the art currently features all over the comuna (Mackenzie, 2018) and its artists such as 'Chota13' and 'YesGraff' have become renowned beyond the confines of La Comuna 13 (Mackenzie, 2018). Their art is now widely recognised throughout the entire city and even worldwide.

E. Lush explains that graffiti is actually illegal in Medellin while there are currently dozens of large-scale murals throughout La Comuna 13 (Lush, 2020). This means that in order for the local artists to create a mural, they need to obtain permission from the area's chief artists as well as the building owner (Lush, 2020). 
This content is unavailable.

Please consult the figure list for further information.
This content is unavailable.

Please consult the figure list for further information.
This content is unavailable.

Please consult the figure list for further information.
This content is unavailable.

Please consult the figure list for further information. 
This content in unavailable.

Please consult the figure list for further details.
This content in unavailable.

Please consult the figure list for further details.

This content in unavailable.

Please consult the figure list for further details.
This content in unavailable.

Please consult the figure list for further details. 


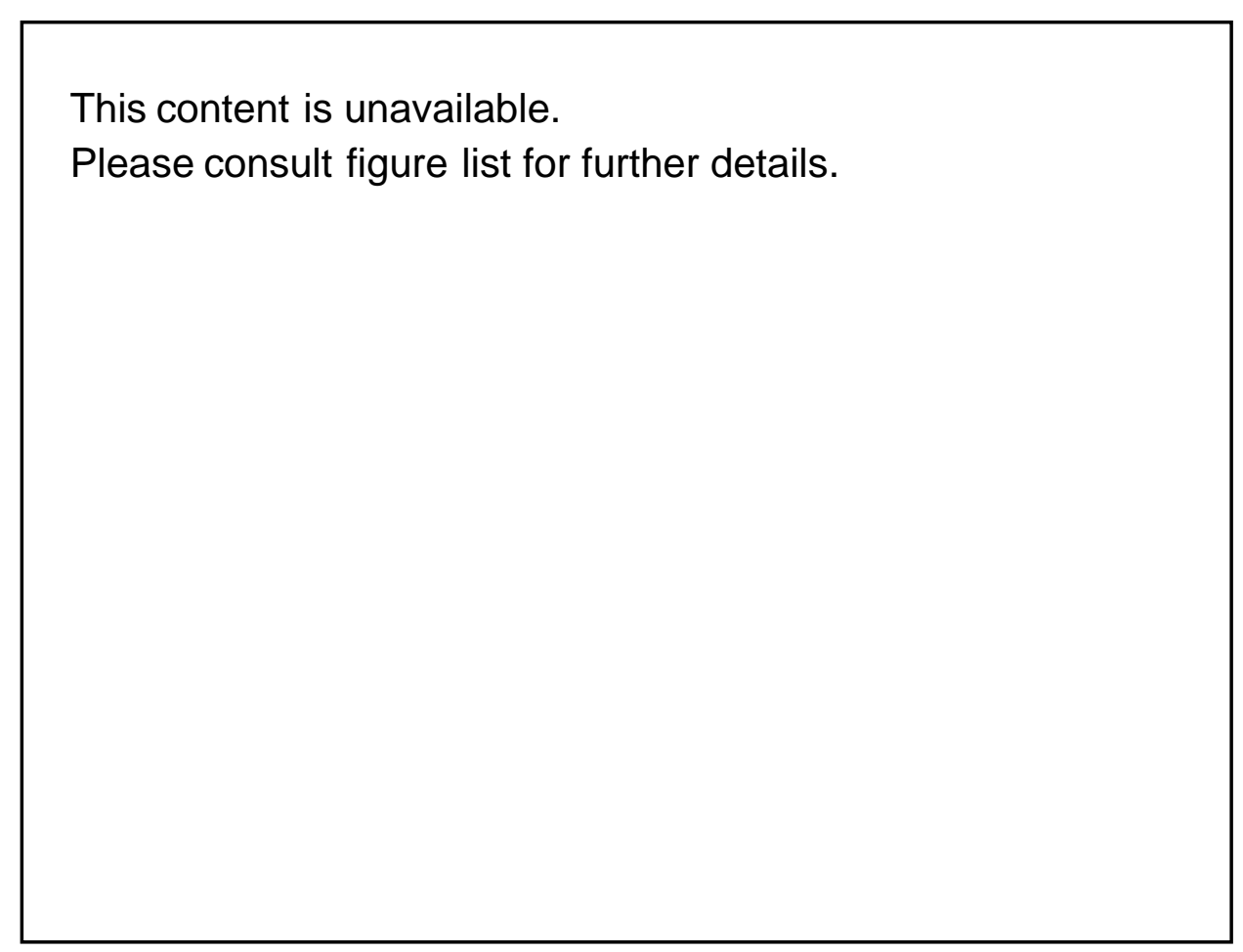

This content is unavailable.

Please consult figure list for further details.
This content is unavailable.

Please consult figure list for further details.
This content is unavailable.

Please consult figure list for further details. 


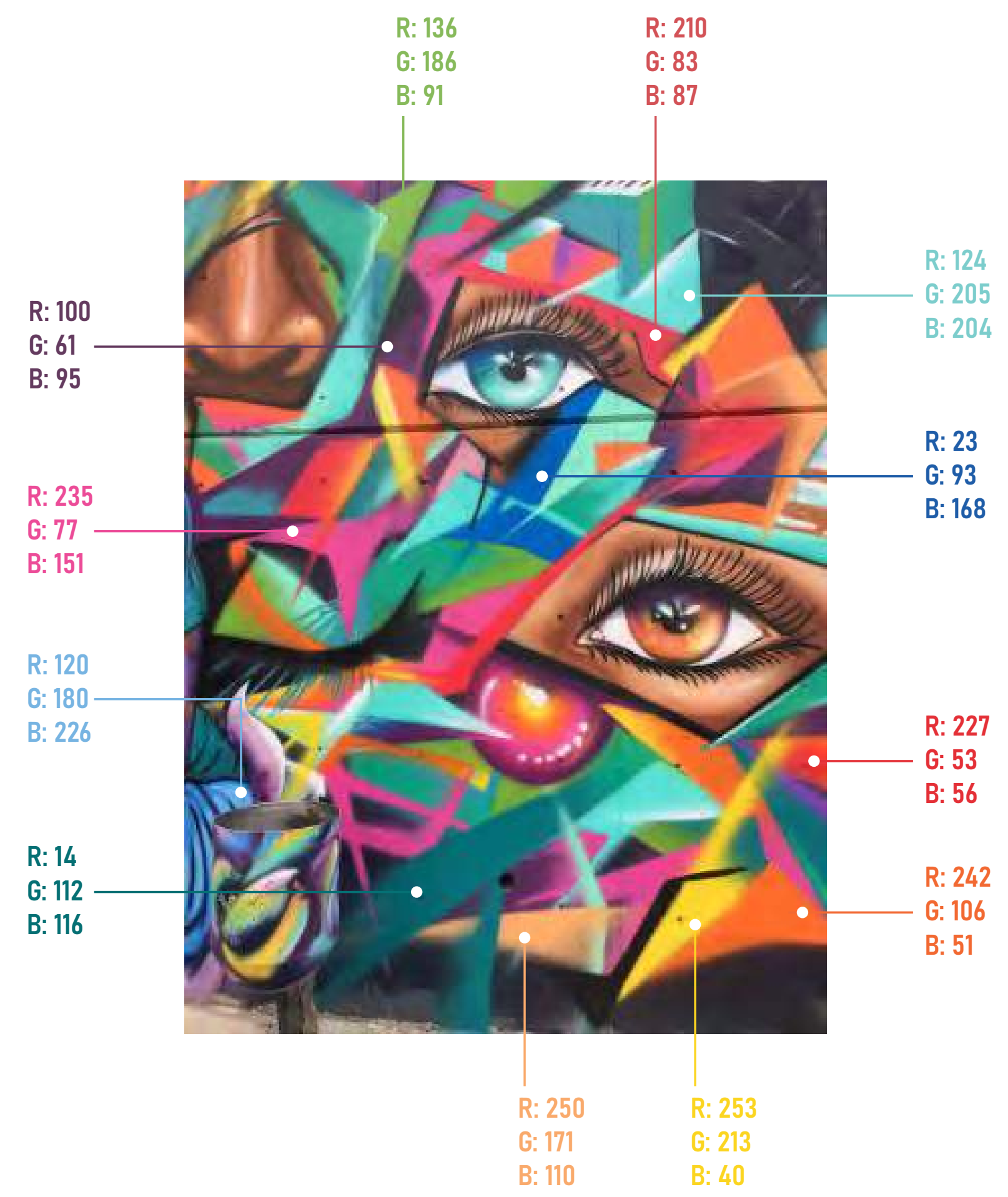




\section{THE EXTRACTION PROCESS}

El proceso de extracción

After becoming familiar with some of the art and analysing it, the process of re-coding and extracting forms from it begins. This development consists entirely of digital drawing processes.

First, the image is re-coded through the drawing of shapes or forms on top of the mural image. This is a purely intuitive process. The idea behind this is to begin to develop some type of forms which could be taken into the concept design stage of this design-led research. A colour palette is created and applied using the colours from the mural. The product of this stage of the extraction process is that the image becomes mostly covered by colourful shapes.

Once the shapes have been created, they are extracted from the image. This involves moving them away from the image and into the page. This begins to create a relationship between the forms and instances such as intersections and thresholds begin to emerge. The product of this stage is different for every mural. Generally, the result is a collection of colourful forms which have been re-arranged into a new pattern and started forming relationships with one-another.

Lastly, the new collection of shapes is also analysed and particular assemblages of shapes are then extracted from the collection of shapes. These are in smaller groups, usually of three to five shapes. The idea of this is to begin to look at interesting relationships, intersections and forms being created to then consider in the concept design stage.

The extraction process has been graphically explained in the following pages. 


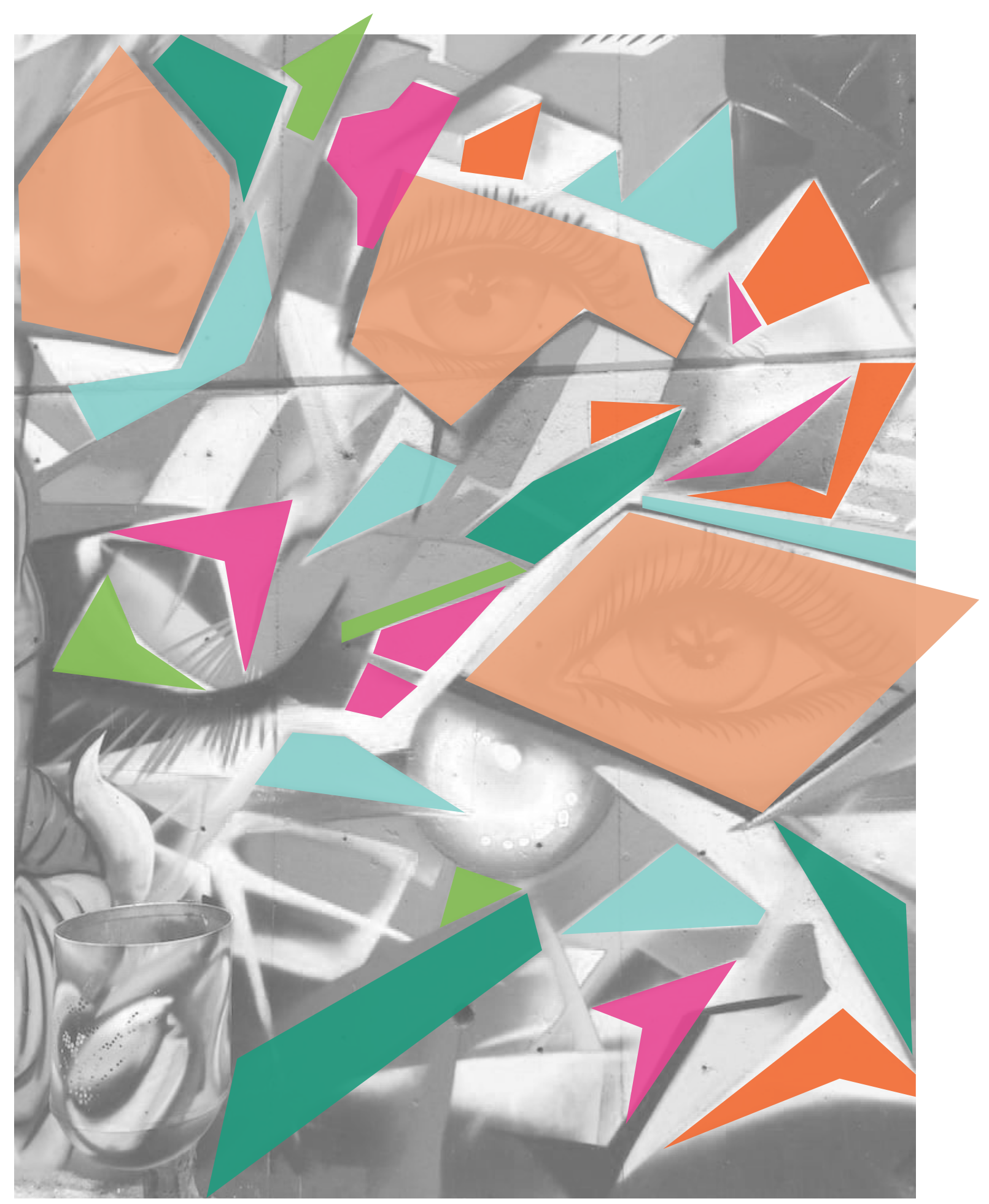

1. THE SHAPES 

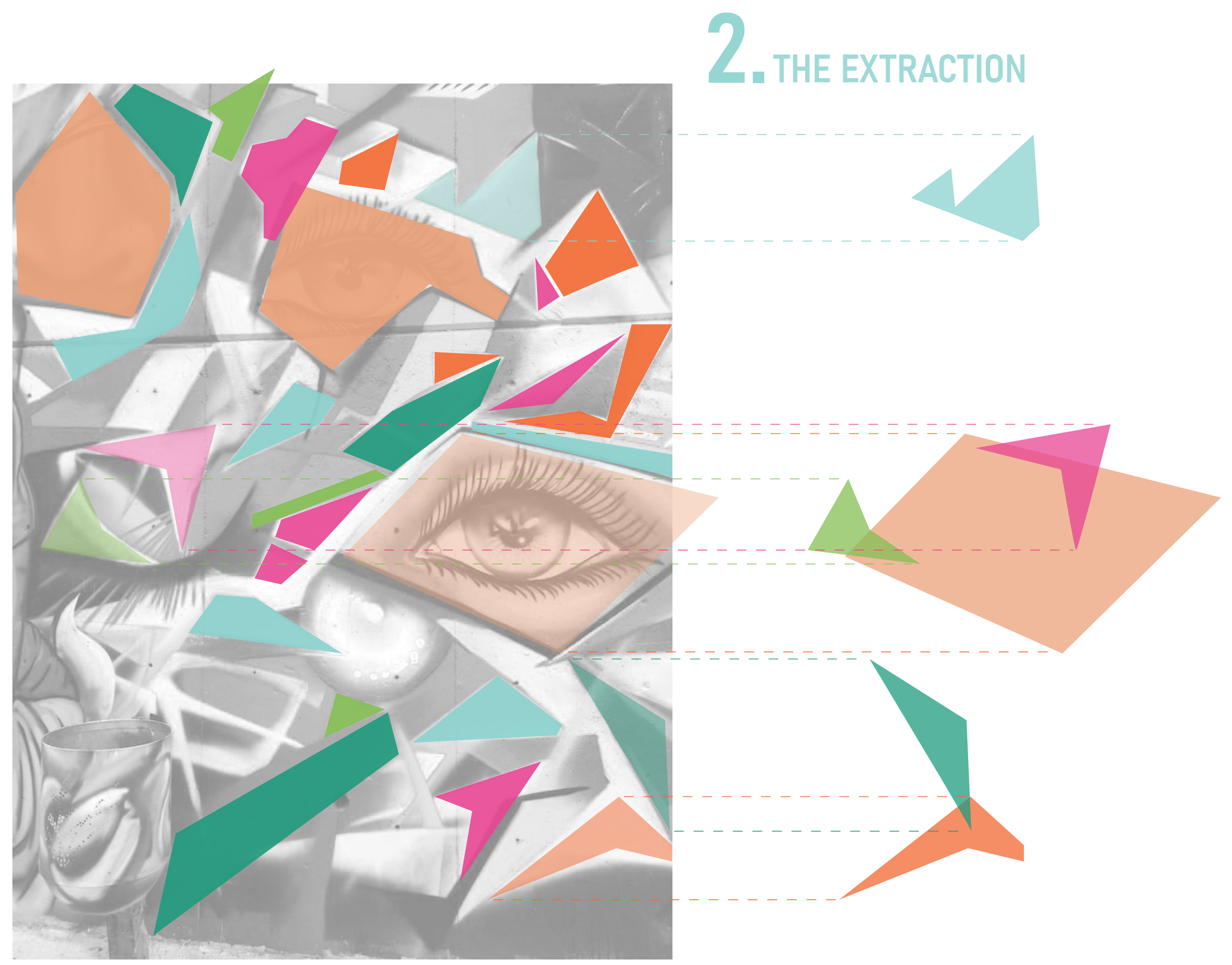
3.
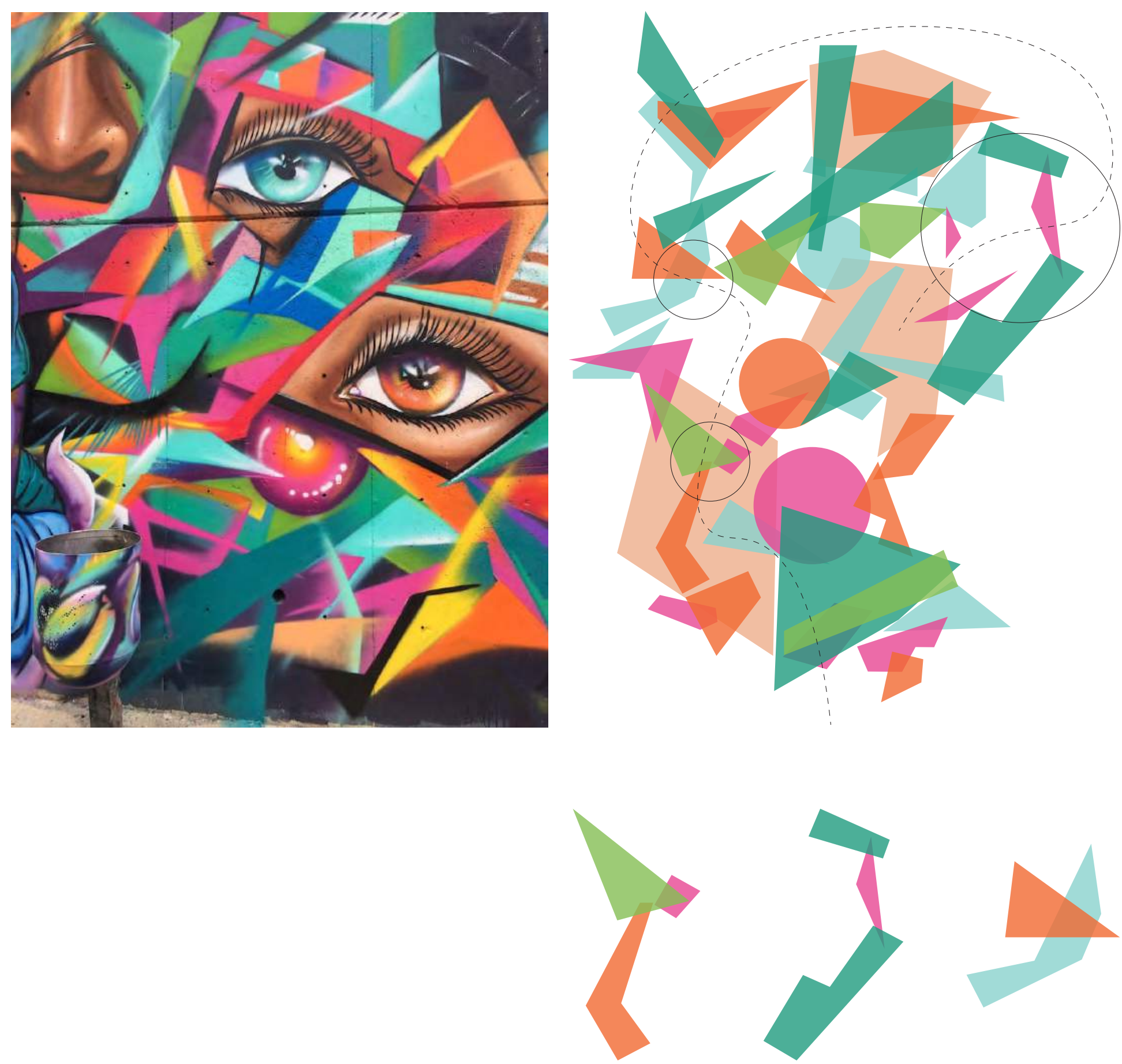

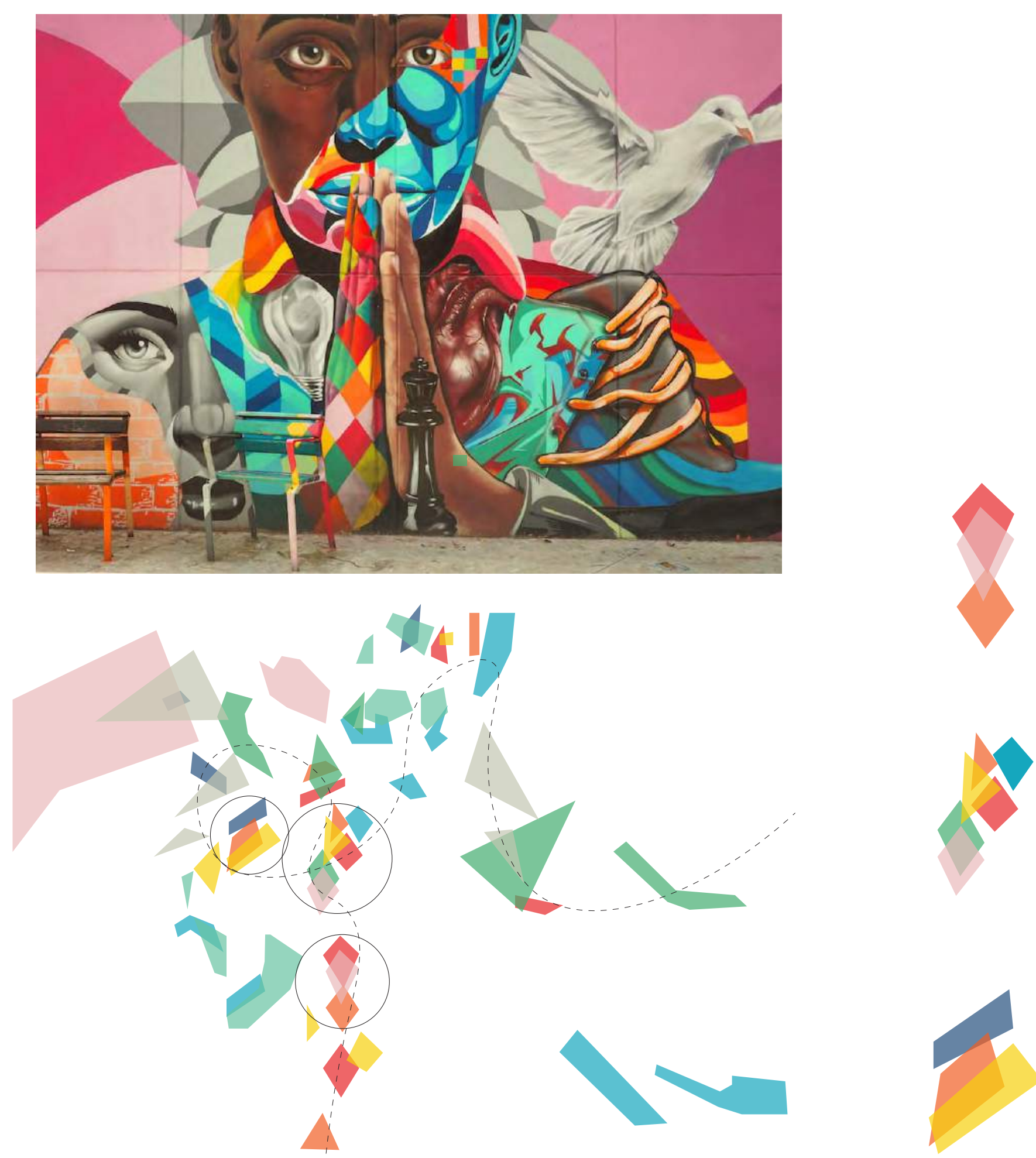

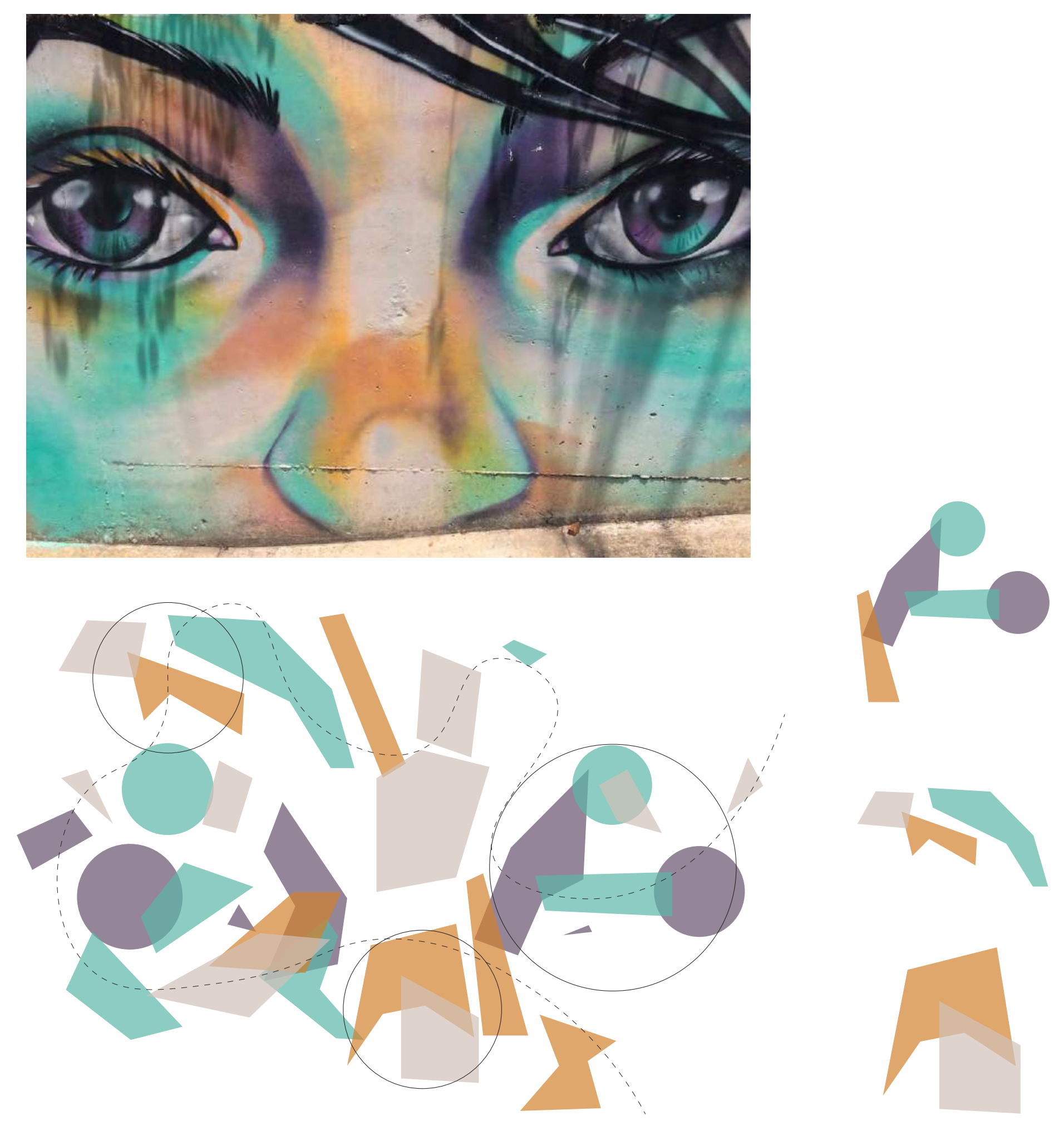

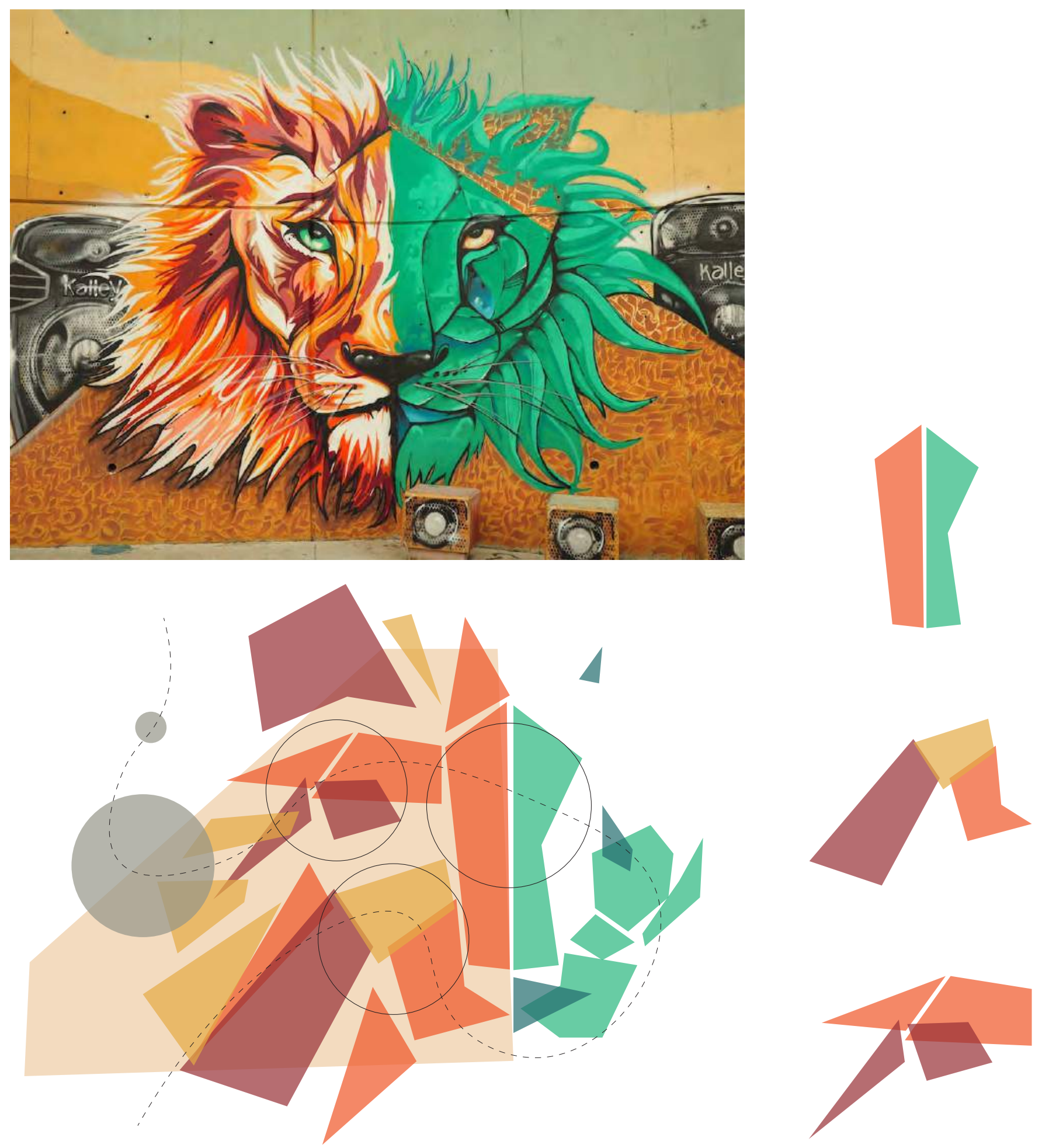


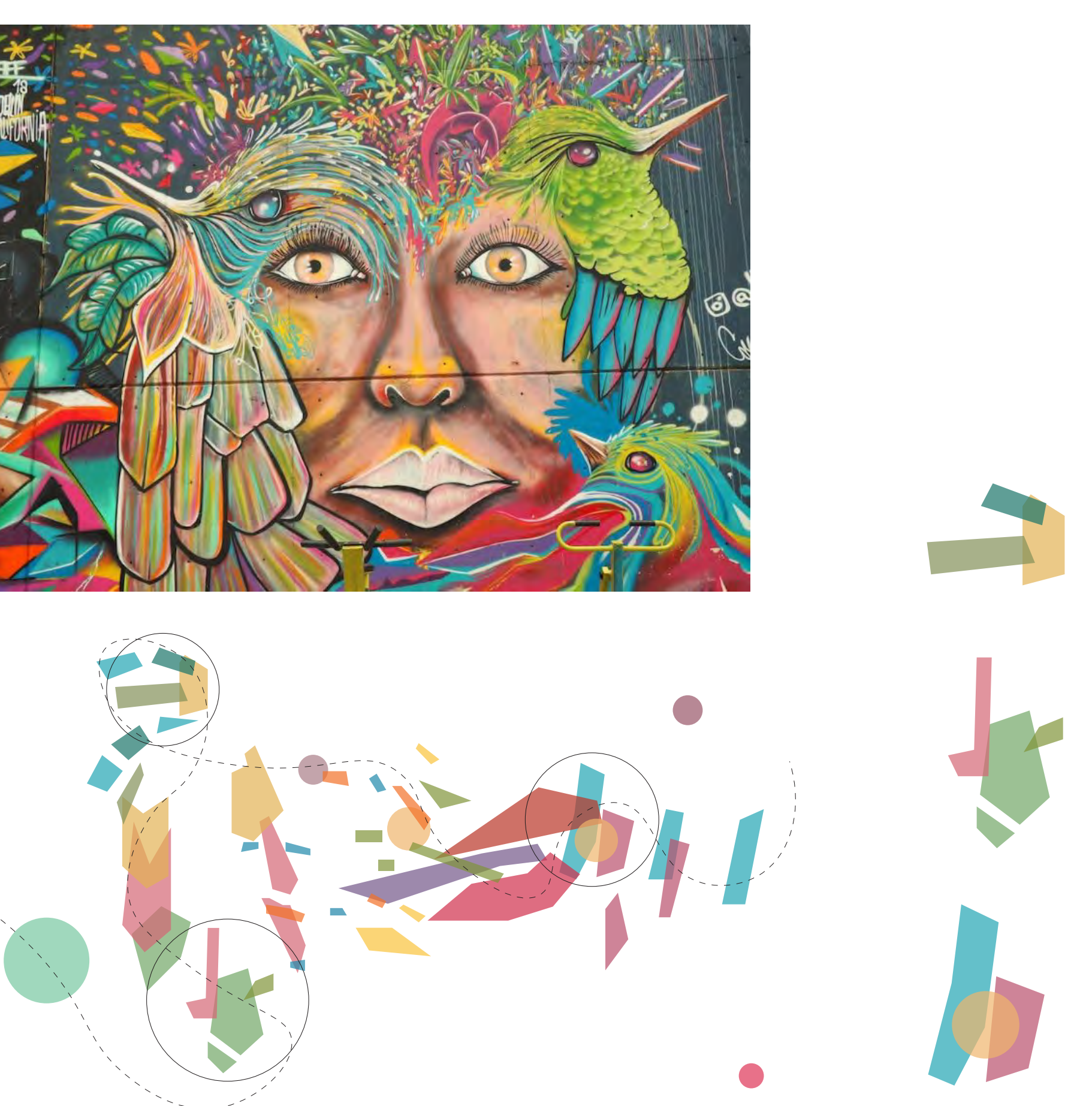



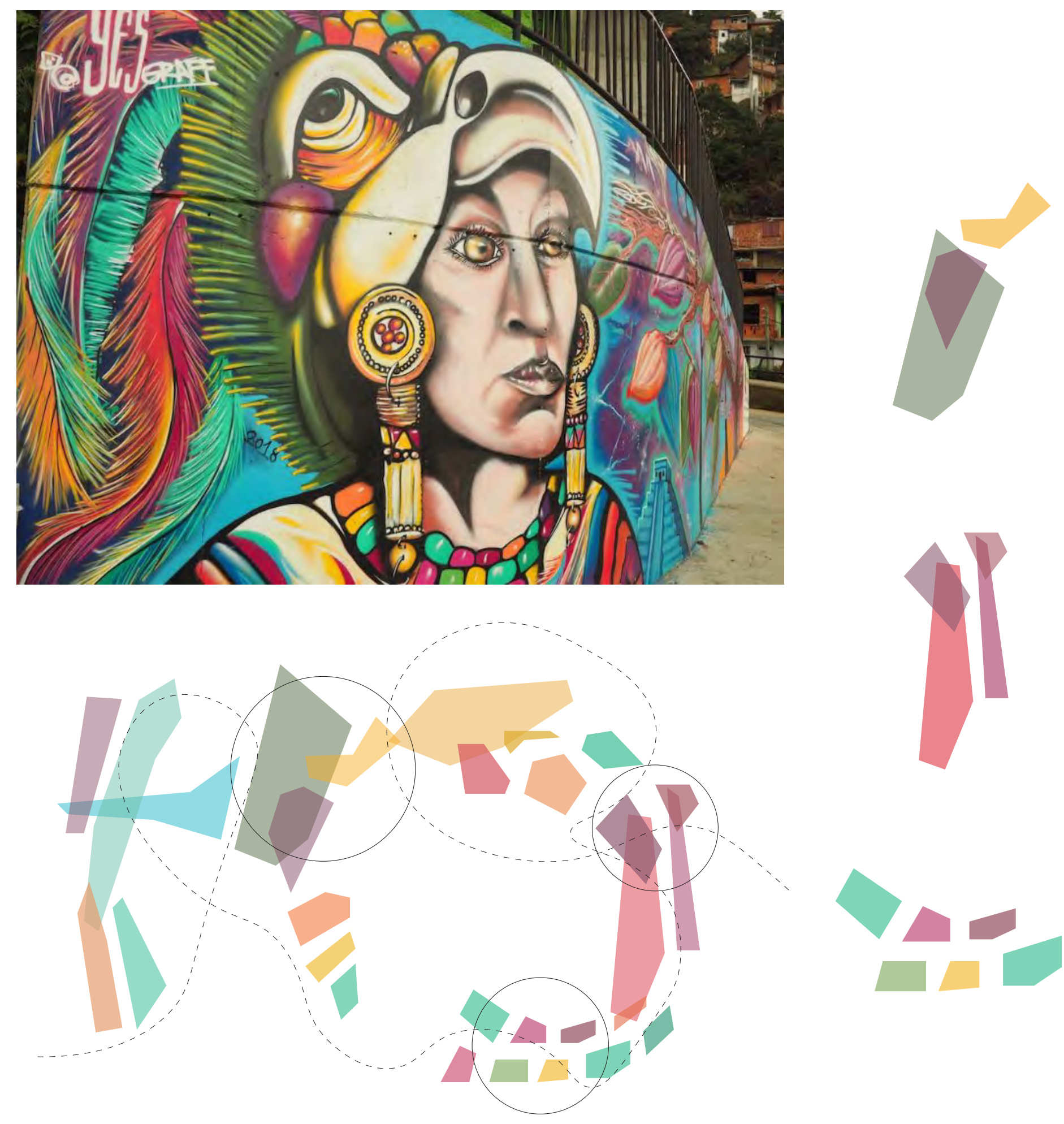


\section{CHAPTER 5: THE ENCOUNTERS}

Capítulo 5: Los encuentros 



\section{A FORCED ENCOUNTER}

CONCEPTO 1: ENCUENTRO FORZADO 


$$
4
$$

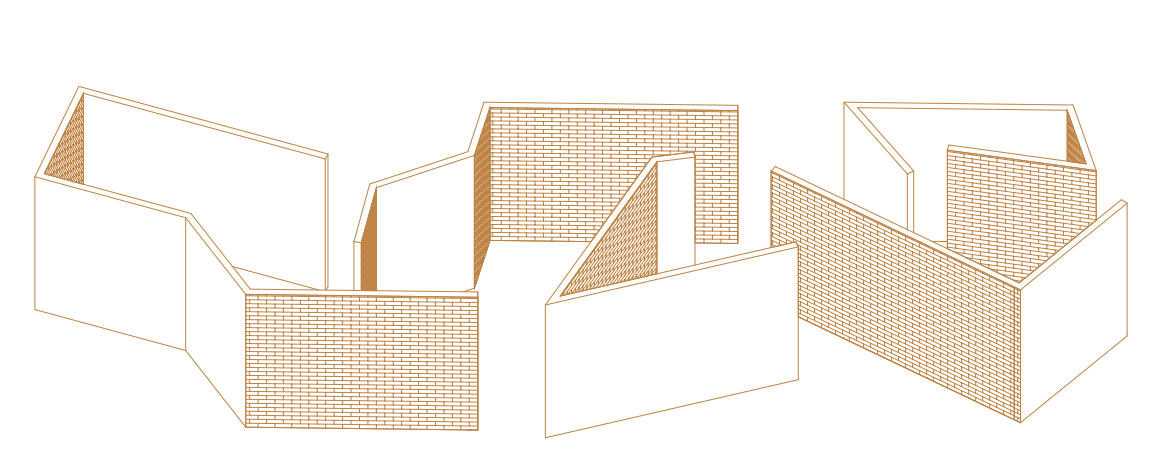

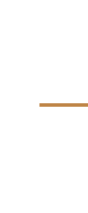




\section{Concept One Introduction}

\section{Introducción al concepto uno}

The first concept begins with an idea: the wall is a canvas for the artistic expression of painting.

One of the aims of this design-led research is to find ways through architecture to bring La Comuna 13's expression from the hilly outskirts of the city, closer to the city centre. Showcasing this community to a wider public whilst also giving its members a place to create art. This aligns with the ideas surrounding identity which were outlined in chapter two and to further support the current intentions of the local government to support this community. By creating walls acting as canvases for the community' expression within the city centre, will perhaps communicate or initiate a sense of belonging to the wider city.

The intention behind the first concept is to create an urban installation which inhabits a frequently used path such as an alleyway or pedestrian walkway, interrupting people's routes by forcing them to walk through the installation. It could be interpreted as an interruption or a provocation. The author views this strategy as appropriate to the cultural context where provocation is an accepted tool to draw attention, to open a conversation.

The development of the design was heavily influenced by the idea of layering which has been previously discussed. First there is the physical or literal layering created by multiple walls on the site and the act of creating the art on the wall. Second the phenomenal layering created by bringing in this community's stories and memories to a new area of the city and layering them on top of what already exists here. There is thirdly a sort of metaphorical layering or merging of this community with the wider city through the architecture but also the art and its significance.
The design process for the installation begins with the study of precedents to create a basic understanding of the possibilities of an urban installation but also to gain some inspiration. From this, the drawing process begins and is used not only to develop the design but also as a research tool. Whilst drawing, it becomes obvious that seeing the walls as a collection of planes enables a sort of freedom in terms of creativity because they are not seen as these rigid architectural elements but as a much more flexible design element. They are freed from having to embody any expectations of what a wall should be and instead they are filled with prospect. They can be seen as a canvas to be used by the community and as a gallery of sorts as people move through the installation. The arrangement of the walls on the site was driven by ease of circulation. This meant understanding how these walls were going to guide people through the installation and what sort of affordances they were going to create for the viewer as they move through. 
“... Ese arte se convirtió en la posibilidad de decir 'estamos presentes', aquí estamos y vamos a decirle a esta ciudad que nosotros existimos." - Kbala

"...Art became the possibility of saying 'we are present', here we are and we are going to tell this city that we exist." 
This content is unavailable.

Please consult the figure list for further details.

Figure 25

\section{Passage by Alfredo Baladron}

This is a temporary installation located in Logrono, Spain. The structure adorns the chimney of the Tabacalera, in a space between dividers. The aim of this exhibit is to change the character of the space by hosting it and inviting viewers to take a seat and enjoy their surroundings.

This installation alters the way in which people move through a circulatory space and could be seen as an act of provocation. It forces them to appreciate it in a completely different way and take in the beauty which surrounds them. These are both ideas which could be taken forward into the design of the urban installation as the idea is to physically force people to stop and notice as they move through.
This content is unavailable.

Please consult the figure list for further details.
This content is unavailable.

Please consult the figure list for further details.

Figure 26 


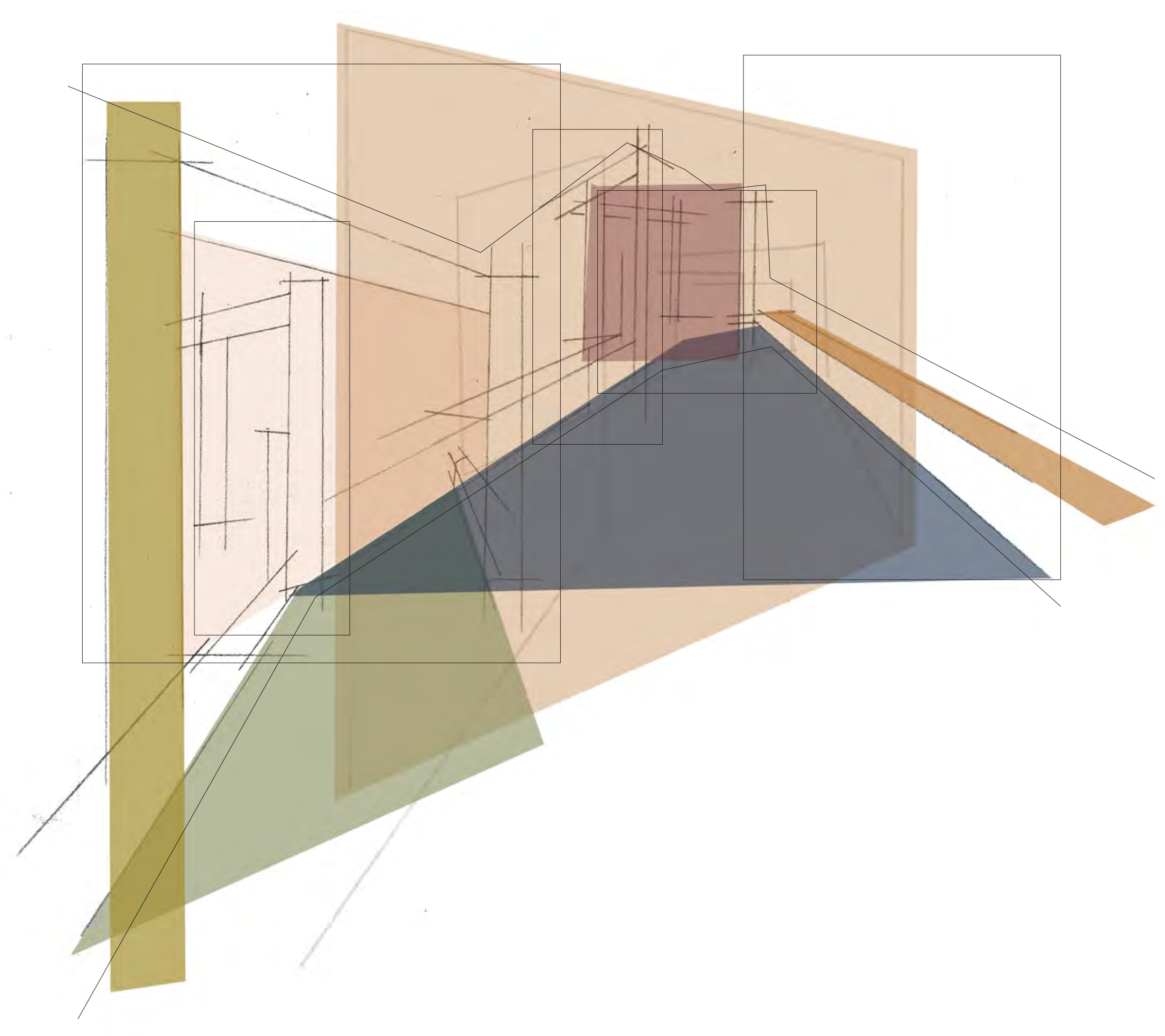




\section{City Thread by SPORTS}

City Thread is a public space, installation and structure located in Chattanooga, United States. This design won the Passageways 2.0 international design competition to turn a formerly unused and neglected alley into a vibrant public space. The installation consists of a 153 metre continuous linear steel tube and painted graphic surfaces. The idea is that this project physically connects visitors and locals with a single gesture while supporting various programmatic possibilities and activities. The linear structure and graphics imply a variety of smaller spaces within the alley, breaking down the overall space into a series of more intimate spaces, or "urban rooms".

Some of the ideas displayed in this design become interesting to consider such as the idea of connection but also creating smaller and more intimate spaces throughout. There appears to be a strategy of a fringe community interacting with a wider community. In the design of the urban installation in this design-led research there will be a connection created between La Comuna 13 and the wider public by bringing their art to the centre of the city. Then creating a higher visibility and appreciation through the design of the walls which the art is created on.

This content is unavailable.

Please consult figure list for further details.
This content is unavailable.

Please consult figure list for further details.
This content is unavailable.

Please consult figure list for further details. 



\section{Hosier Lane, Melbourne}

Known as Melbourne's most celebrated laneway when it comes to street art, Hosier Lane draws camera-wielding crowds. Many people wanting to be captured posing in front of the edgy graffiti, stencils and art installations which cover almost every available surface. The subject matter consists of mostly political and countercultural content, with some irreverent humour throughout.

This is an outstanding example of how street art can take control of a transitional space such as an alleyway and transform it. There is evidence of layering displayed here but this also exhibits how street art can attract people to a place as simple as an alleyway. It evokes ideas of exposure but also belonging. This aligns with the ideas of giving La Comuna 13 a canvas, allowing them to express themselves completely on this installation of walls and creating an experience for the wider public by locating it directly in their path.

\section{This content is unavailable. \\ Please consult figure list for further details.}

This content is unavailable.

Please consult figure list for further details.
This content is unavailable.

Please consult figure list for further details. 


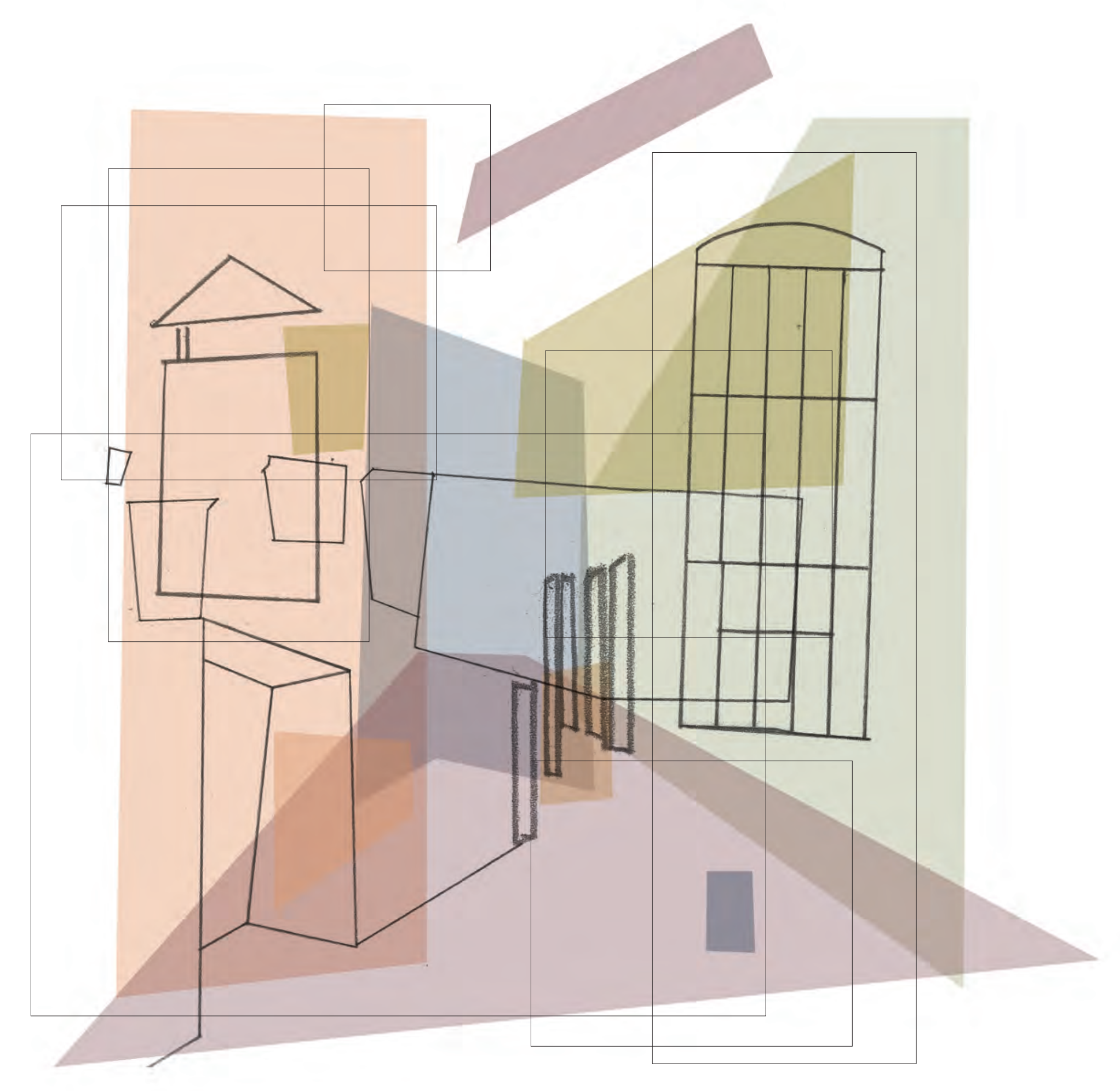




\section{The drawing process}

El proceso de dibujo

After analysing precedents and gaining some inspiration, the drawing process begins through a mixture of digital and analogue formats. A selection of one or a variety of the art interpretation drawings previously exposed (Chapter 4) is complemented by a selected range of shapes extracted from the street art. The selection process consisted of looking at size but also variety in shapes. There is consideration into angles and sometimes proximity.

The author's emergence in the reading of the murals naturally leads to an exploration of the fragmentation of parts.

Once the selection is made, the rearranging process starts. This is done digitally seeing as the art interpretation drawings were originally create digitally. The rearranging process looks at moving and rotating the selected shapes and re-organising them into a new configurations. There are various factors which are considered when it comes to the configuration of the shapes. The first factor is proximity; the shapes are normally brought closer as if to create a sort of alleyway between them. The second factor is the angle of the side of the shape; similarly angled sides will be brought closer to each other or in some cases, be placed opposite to each other. Third is the circulation or connectivity; most of the patterns created tend to create a snake-like pattern. This can be done intentionally but it also happens unintentionally. The directionality of the configuration can be entirely manipulated through the positioning of shapes.

When the new configuration of shapes is finalised the shapes are extruded to create volumes. This is done to begin looking at these shapes as spaces created by vertical planes. The intention is to begin a more meaningful exploration when it comes to ideas of the wall and its implications. This part of the process initiates the examination of spatial qualities and introduces the translation between two-dimensional drawing into volume. This marks the end of the digital drawing processes. A visual representation of the drawing process up to this point can be seen on the following page.

Having this digital drawing of the volumetric interpretation of the street art serves as a template for some analogue drawing. Using butter paper, the drawing is traced and then further developed by introducing the analysis of the location of brick walls. This is further explained in pages 63-68. 
2. ReArRAngment
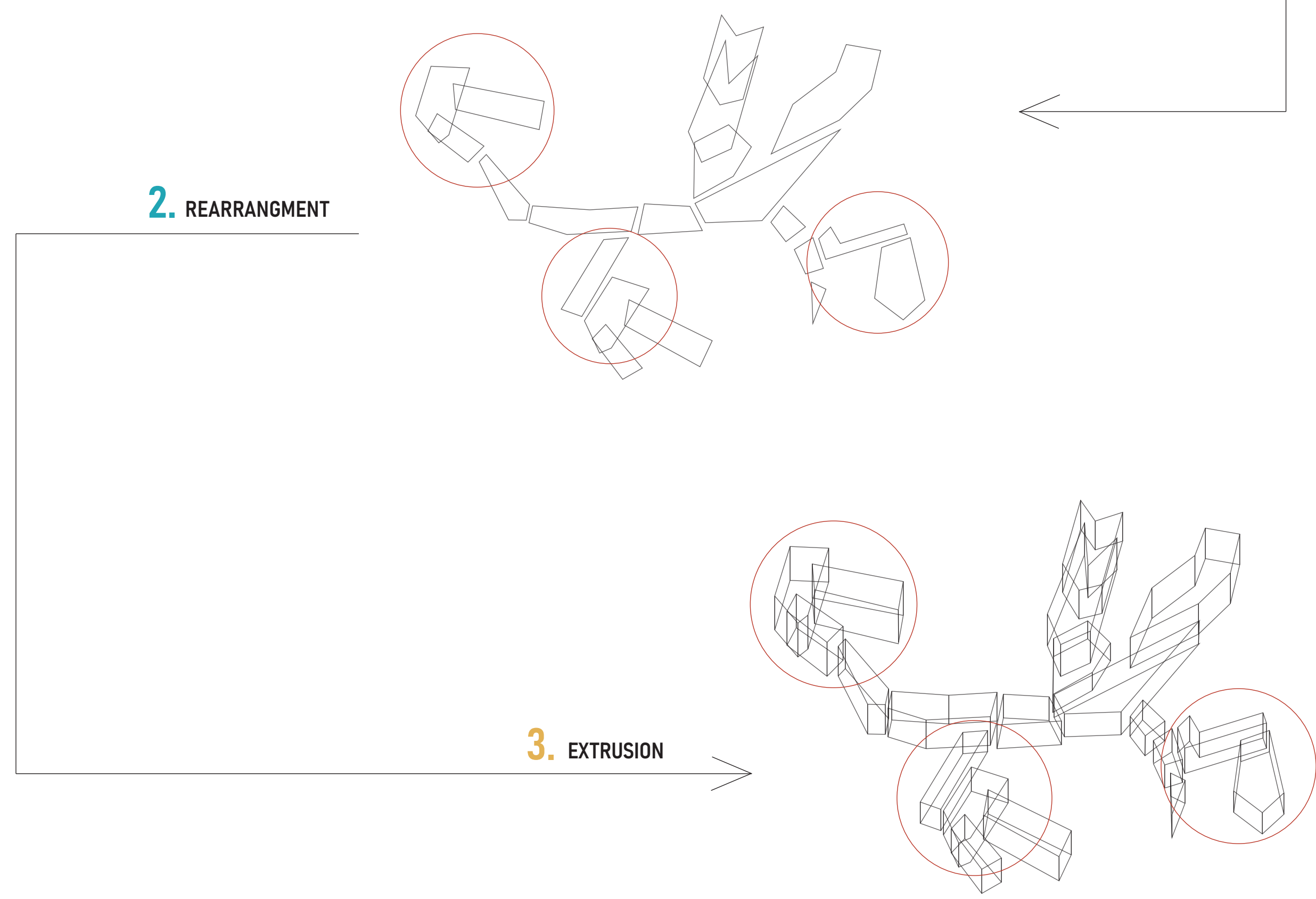

62 
These colourful shapes become an abstracted interpretation of what the community would paint on the wall. The shapes come from the previous studies and breakdown of the art currently created by the community.

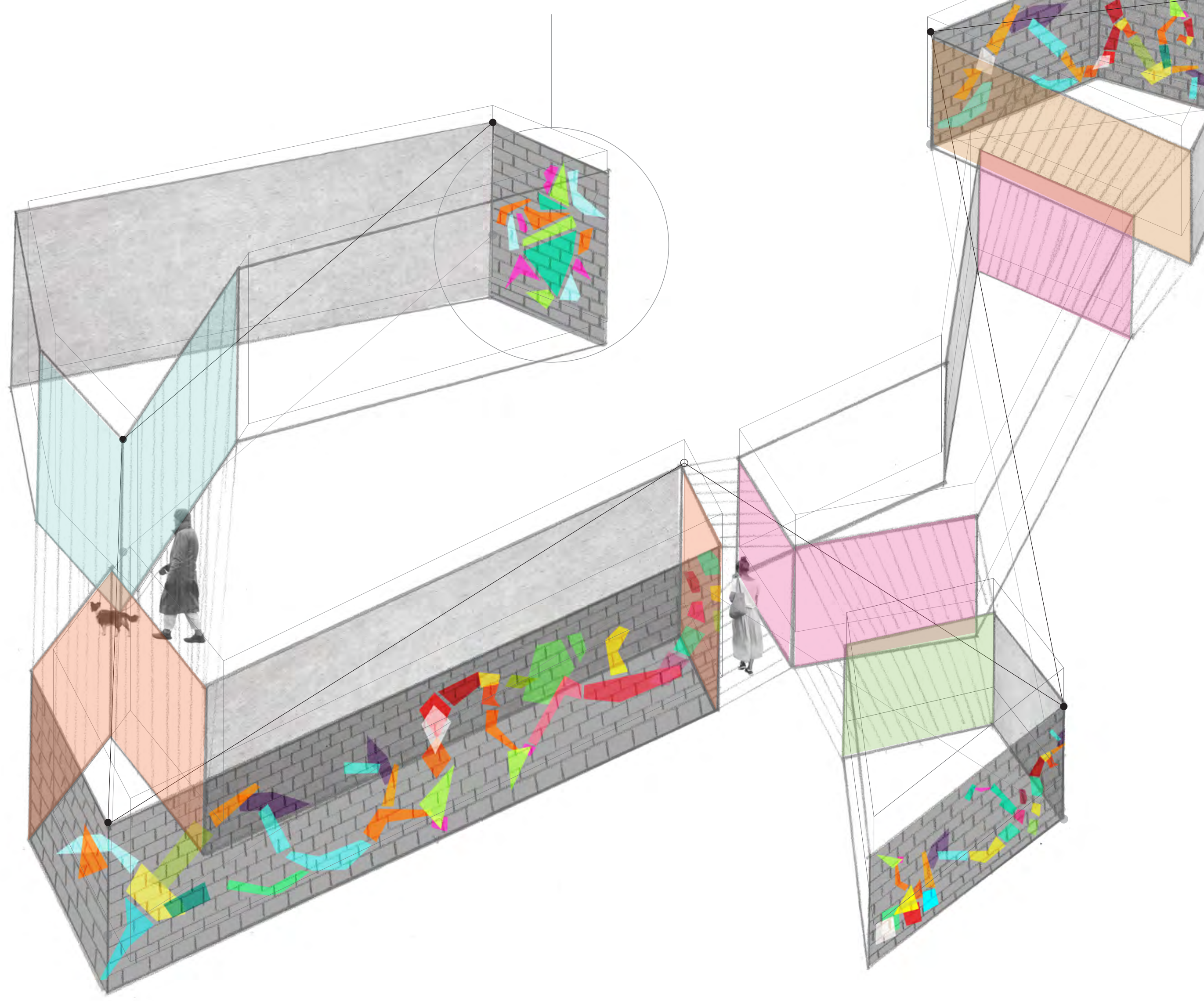




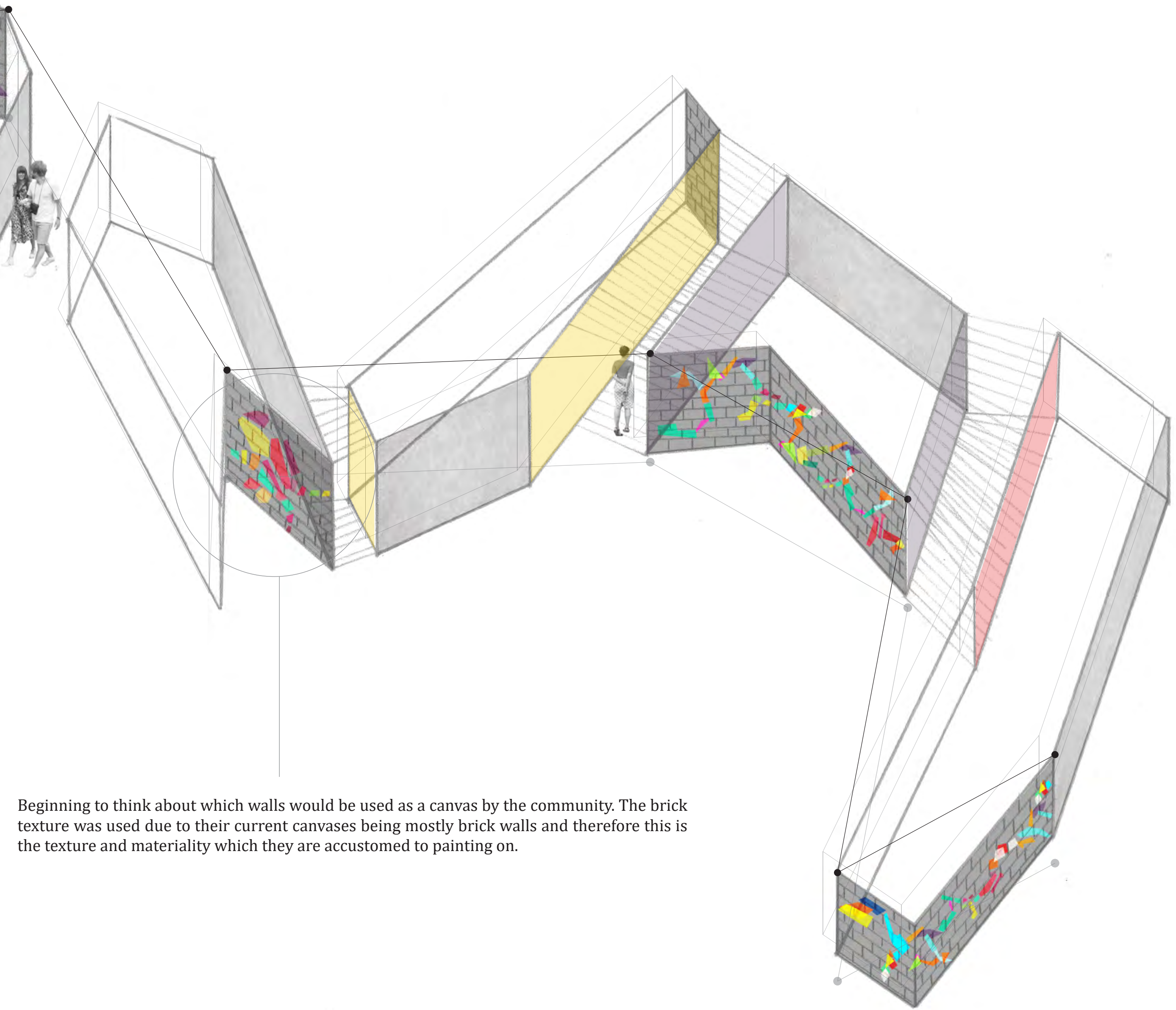


Starting to explore the intersecting of spaces and therefore walls. Looking at what sort of relationships but also spaces are created in these instances. By isolating them, a new series of drawings and concepts begin to emerge.

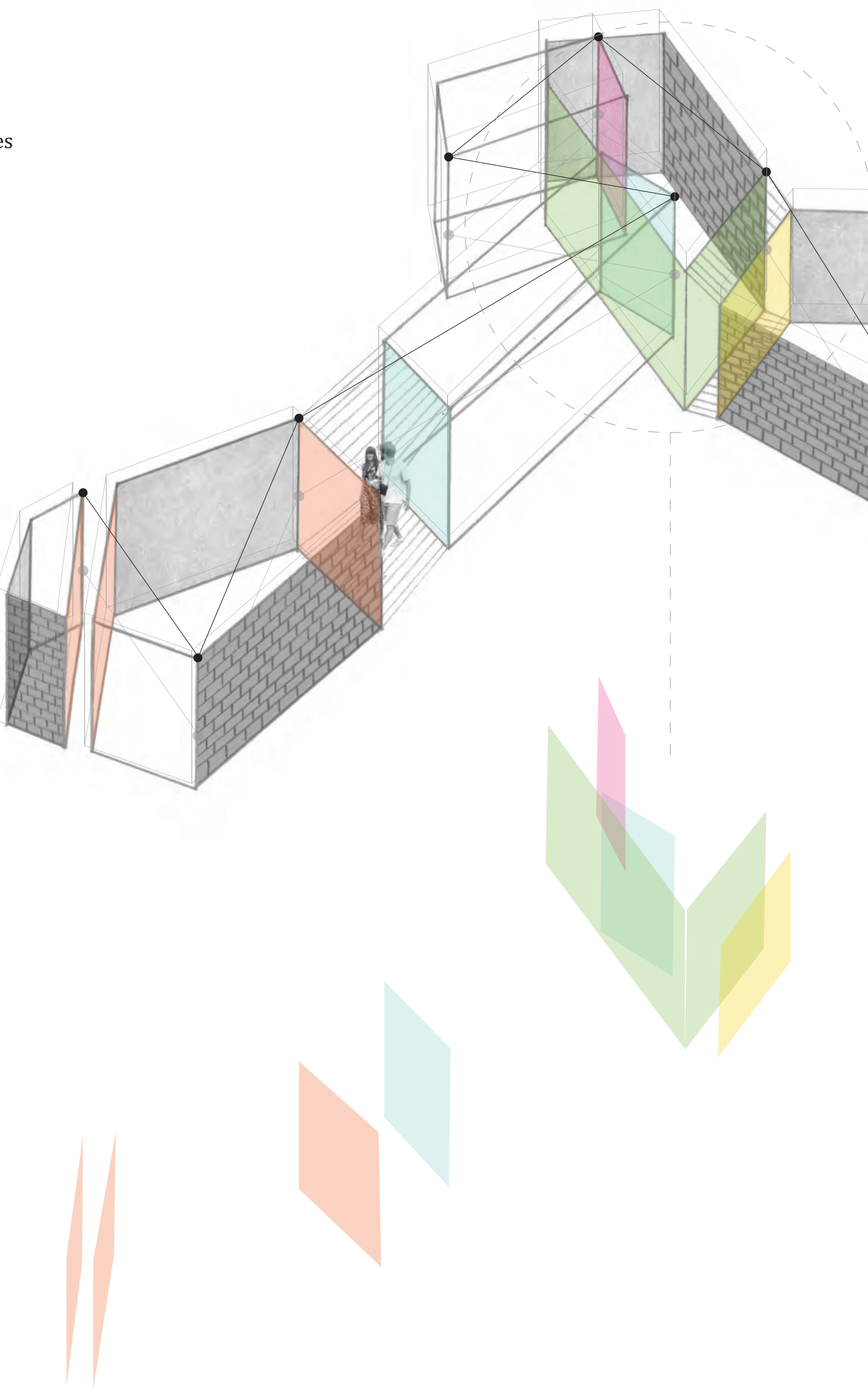



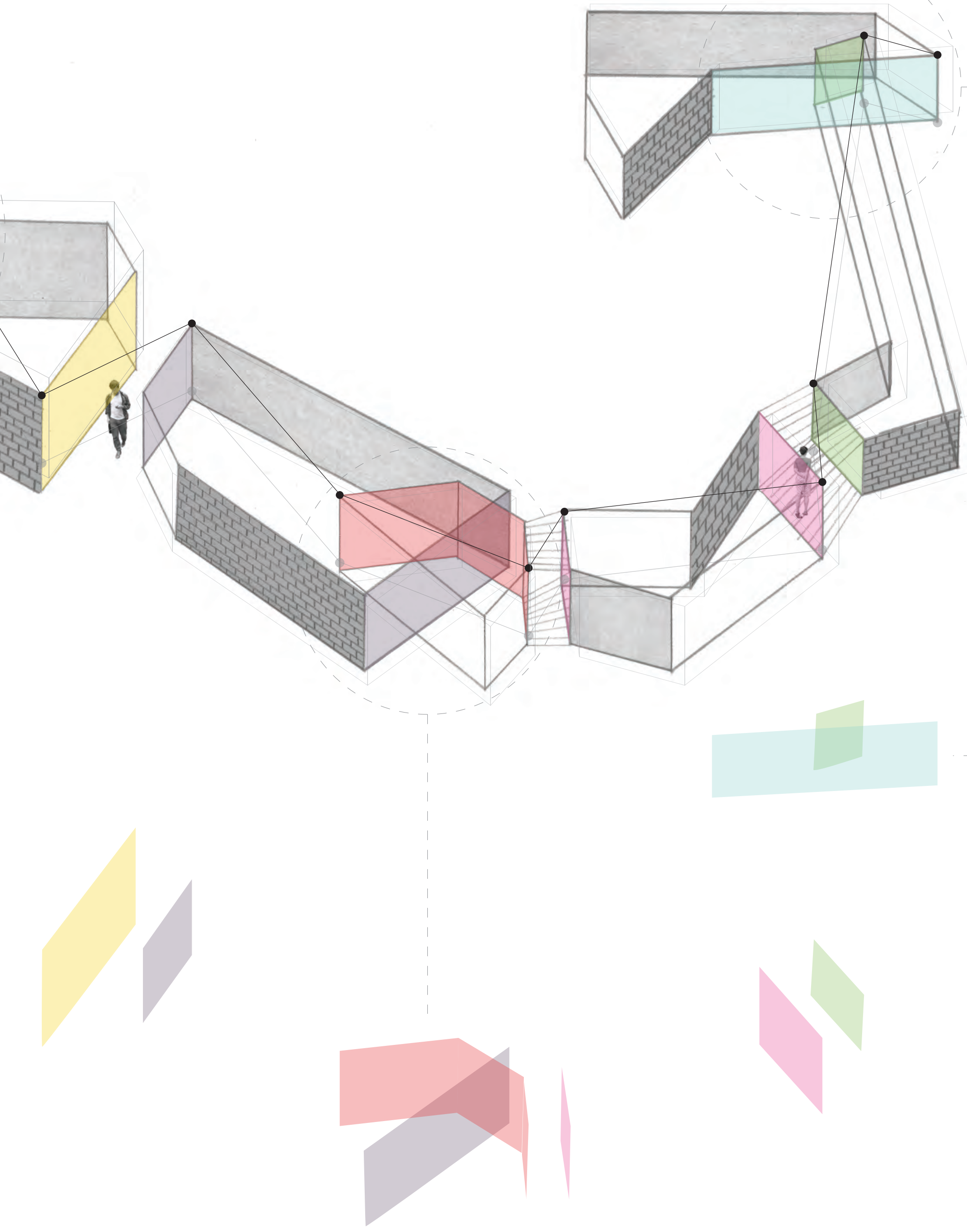



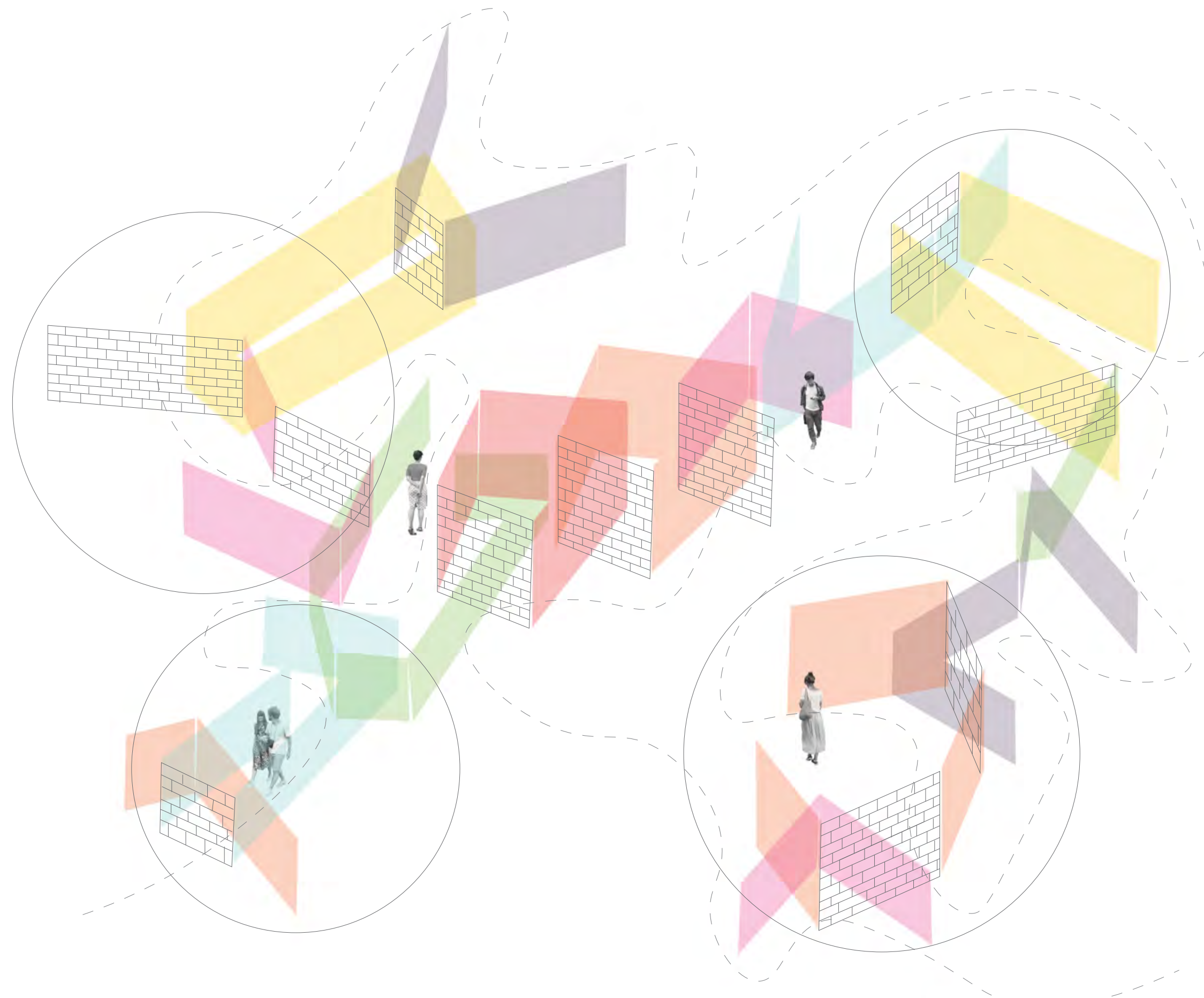

After realising that isolating the intersecting walls is an effective way of conceptualising, a new drawing is created where all walls intersected and the increased number of intersections began to create more defined spaces. This new concept begins to look much more like an installation which people can move through rather than a series of enclosed spaces which all intersect. Continuing to use brick walls is done on purpose in order to highlight their importance as canvas but also to explore their positioning and implications. 


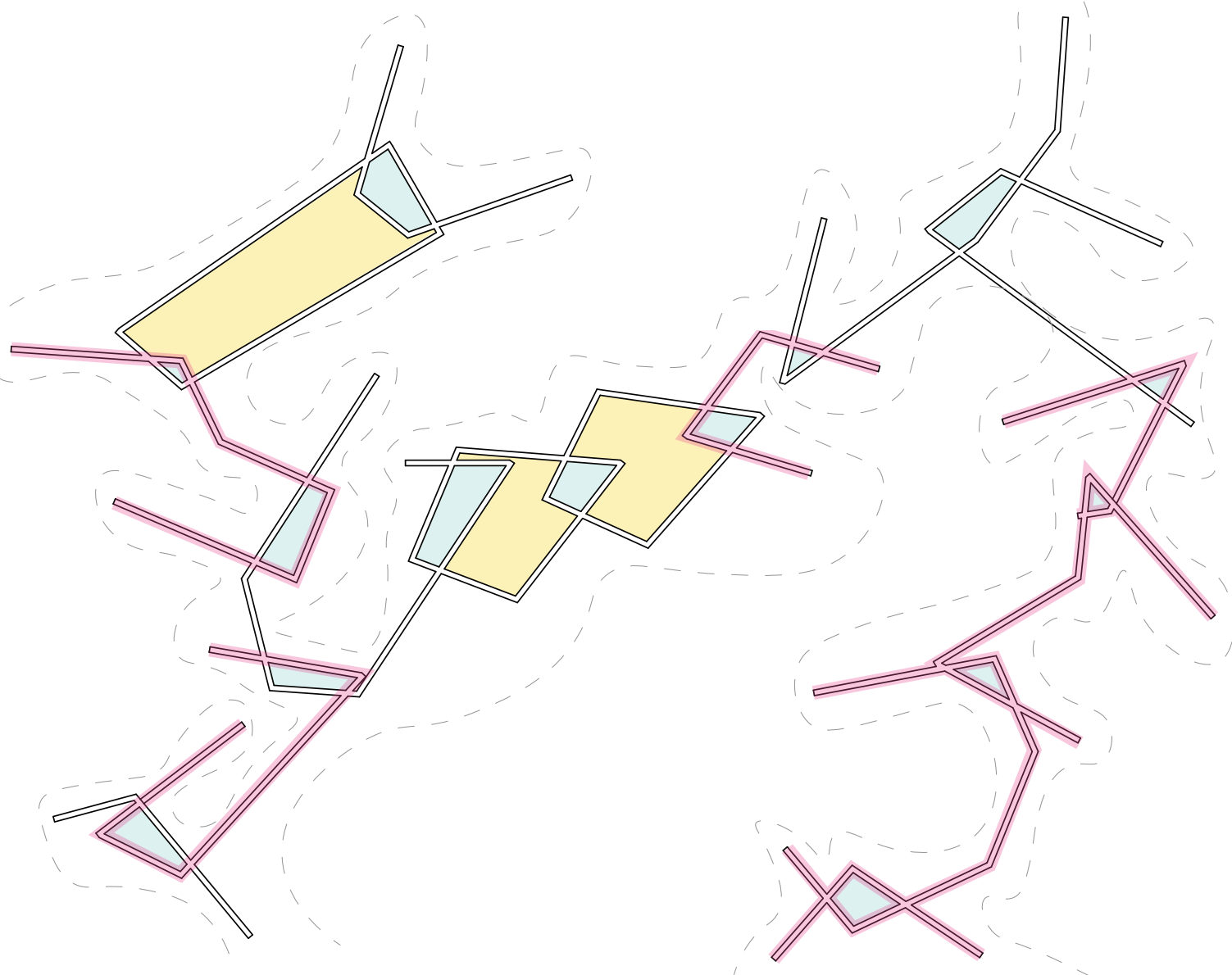

CONCEPT ONE - ITERATION 1 - PLAN
Unusable spaces
Walls chosen for the
development of a new plan
Large enclosed spaces

The next step is to analyse the plan and the effectiveness of circulation and space throughout the design. There is only possibility to move around the walls, but not through and among them. Unused and entirely enclosed spaces become an evident issue. There is also no consideration into site in the creation of the first iteration which will largely affect the design considering the potential sites are pathways and alleyway which are narrow.

It is decided that some of the walls will be taken from this design in order to develop the second iteration of the plan/design.
1.

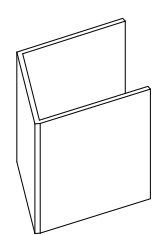

2.

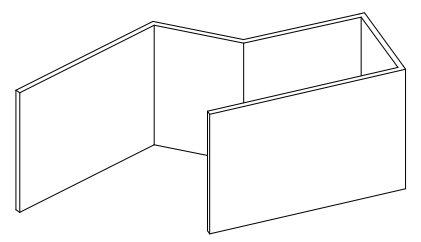

3.

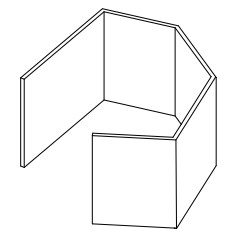

4.

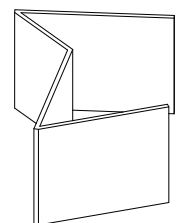

5.

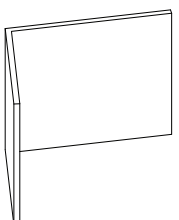

6.

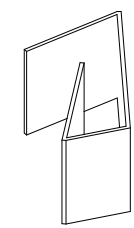

7.

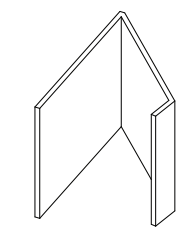

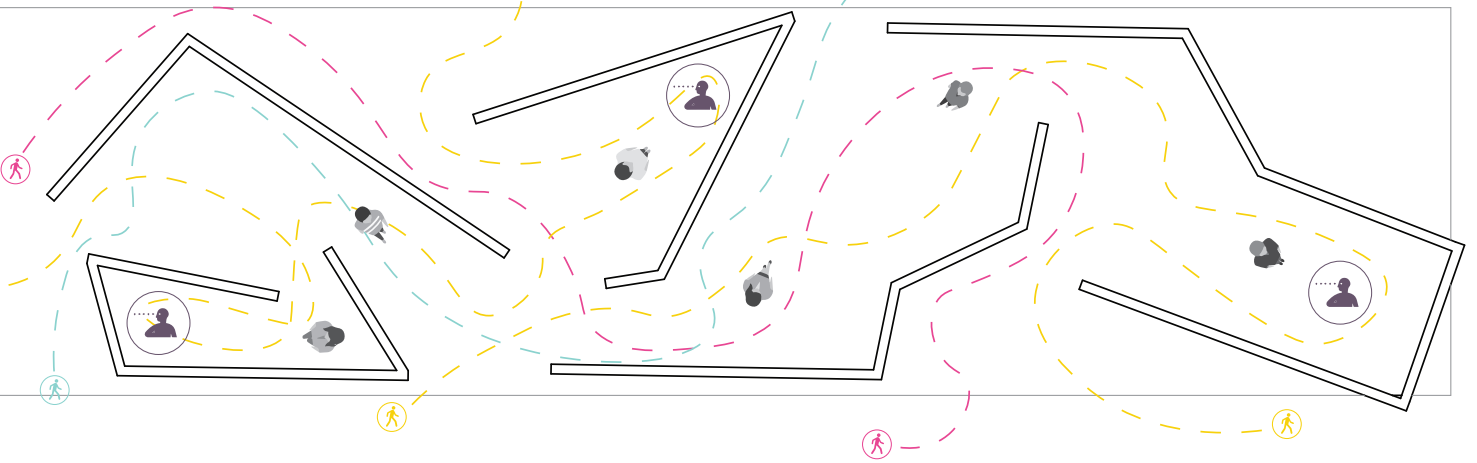

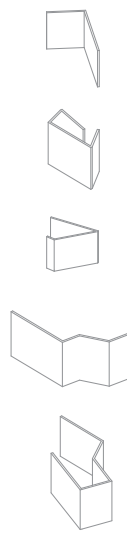

In the development process of iteration two, the selected walls have to be slightly altered to fit the considered site. The number of walls is decreased and circulation is one of the main drivers to ensure that the users have alternative routes of movement throughout. 


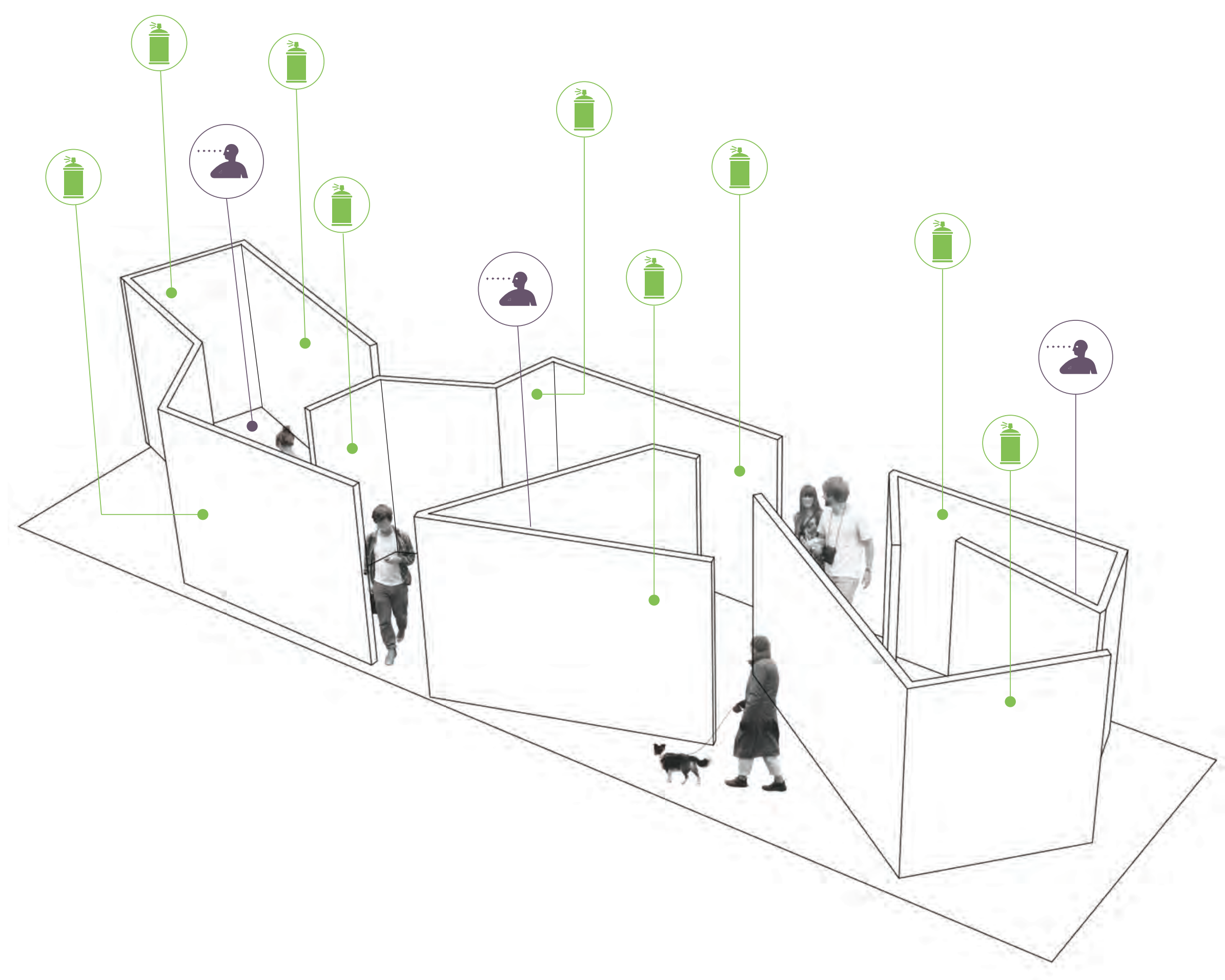




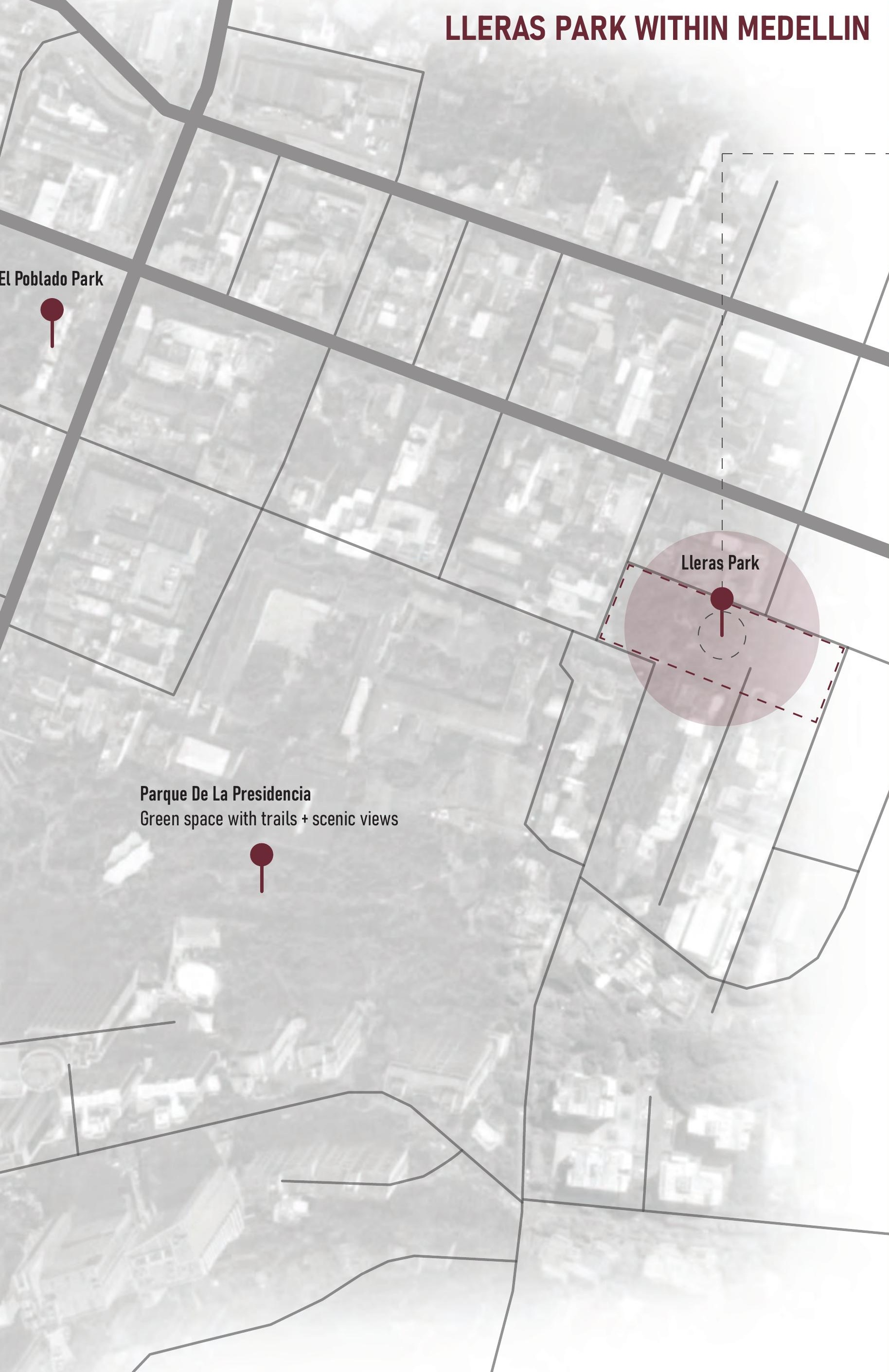

\section{POTENTIAL SITES WITHIN LLERAS PARK}

This content is unavailable.

Google maps street view imagery.

1.

This content is unavailable.

Google maps street view imagery.

2.

This content is unavailable.

Google maps street view imagery.

3. 


\section{WALLS ON SITE}

Lleras Park is chosen as a site due to it featuring some paths and walkways with heavy foot traffic. There is a park with lots of greenery and places to sit. These walkways move around the park and lead into the park, so they are a connection of sorts.

The idea of this forced encounter is to use the vertical plane to interrupt people's views and experiences of a space. Placing the walls directly in their path means there is no other choice but to move through them and experience the art created by La Comuna 13.
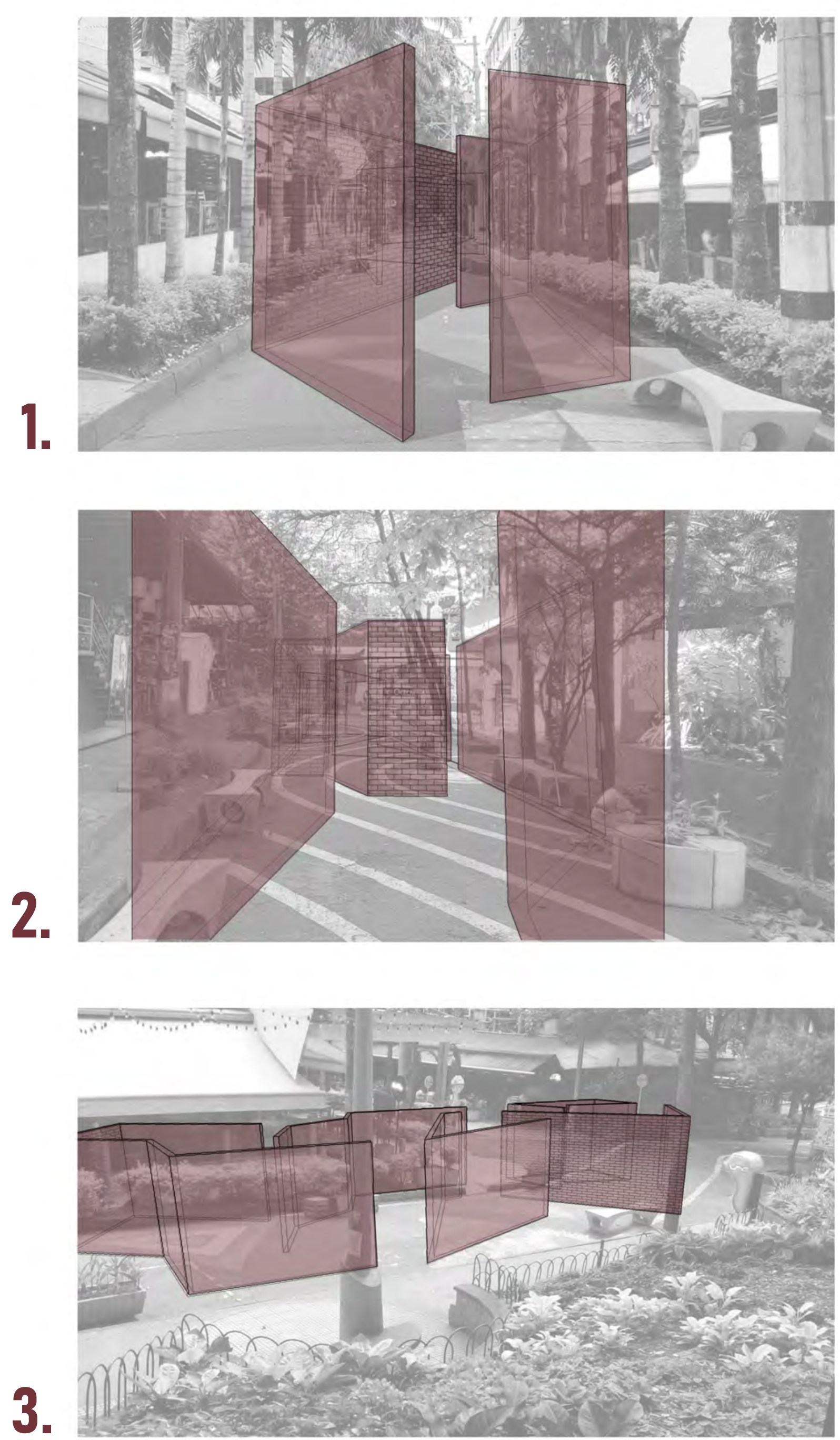


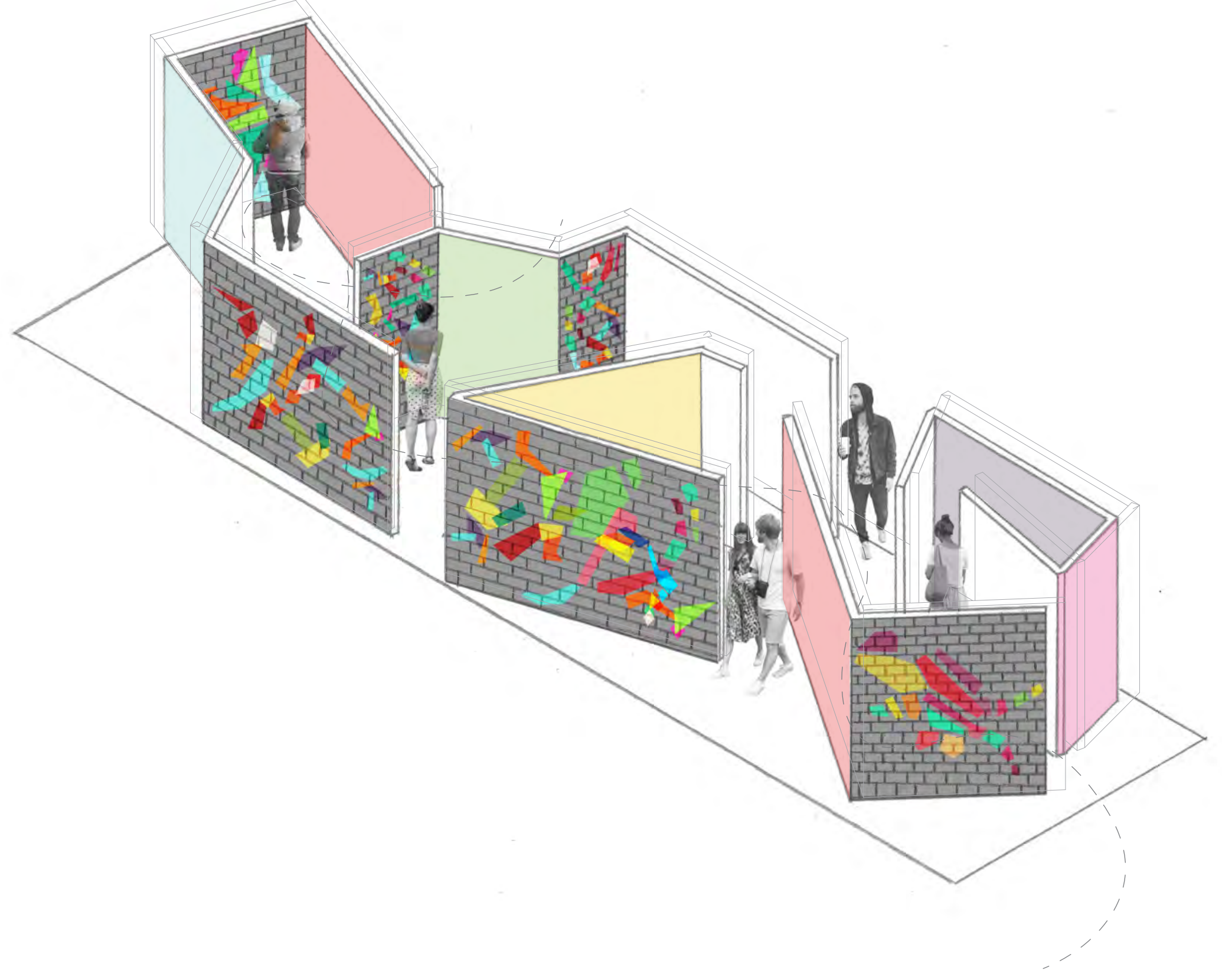





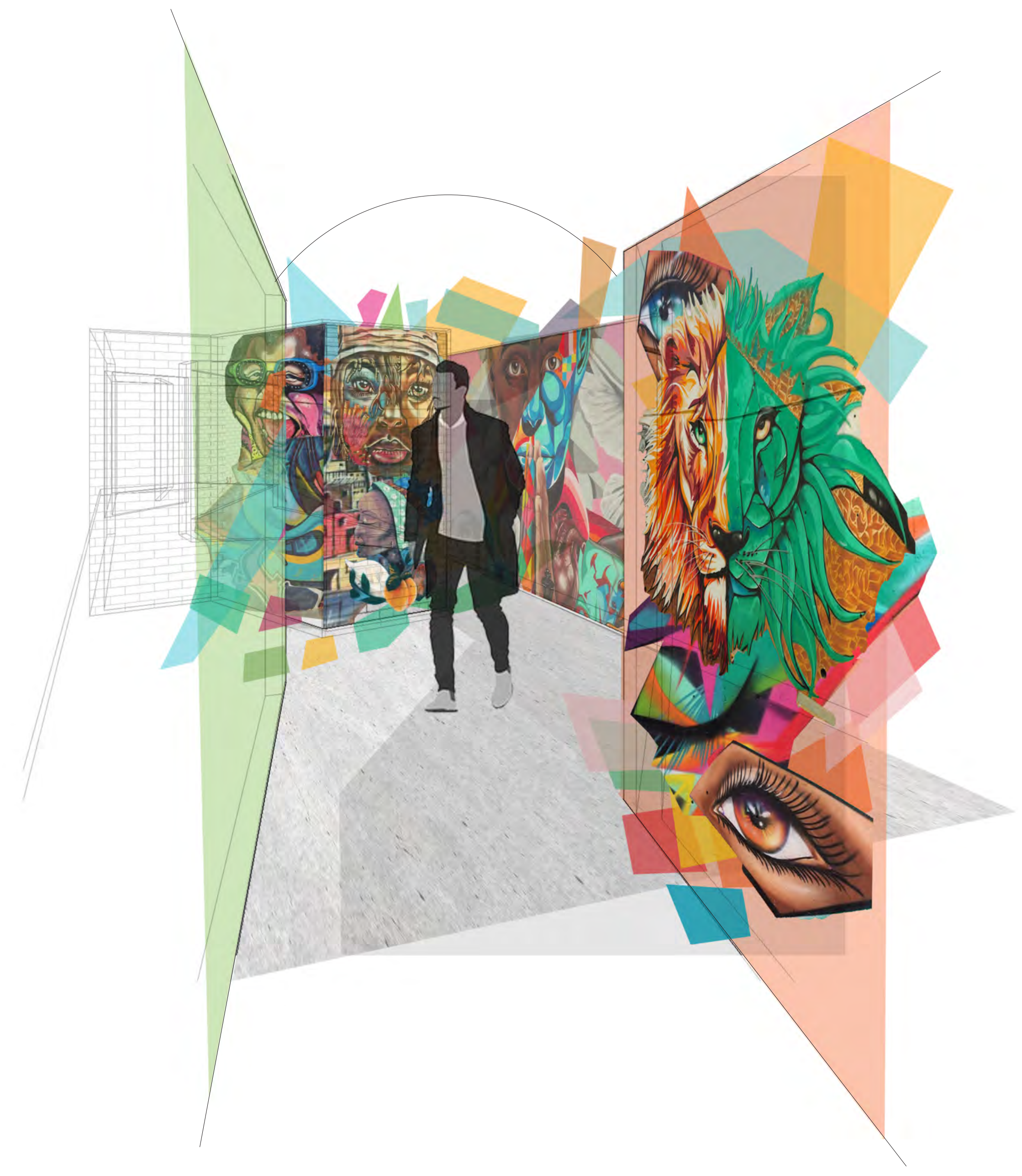




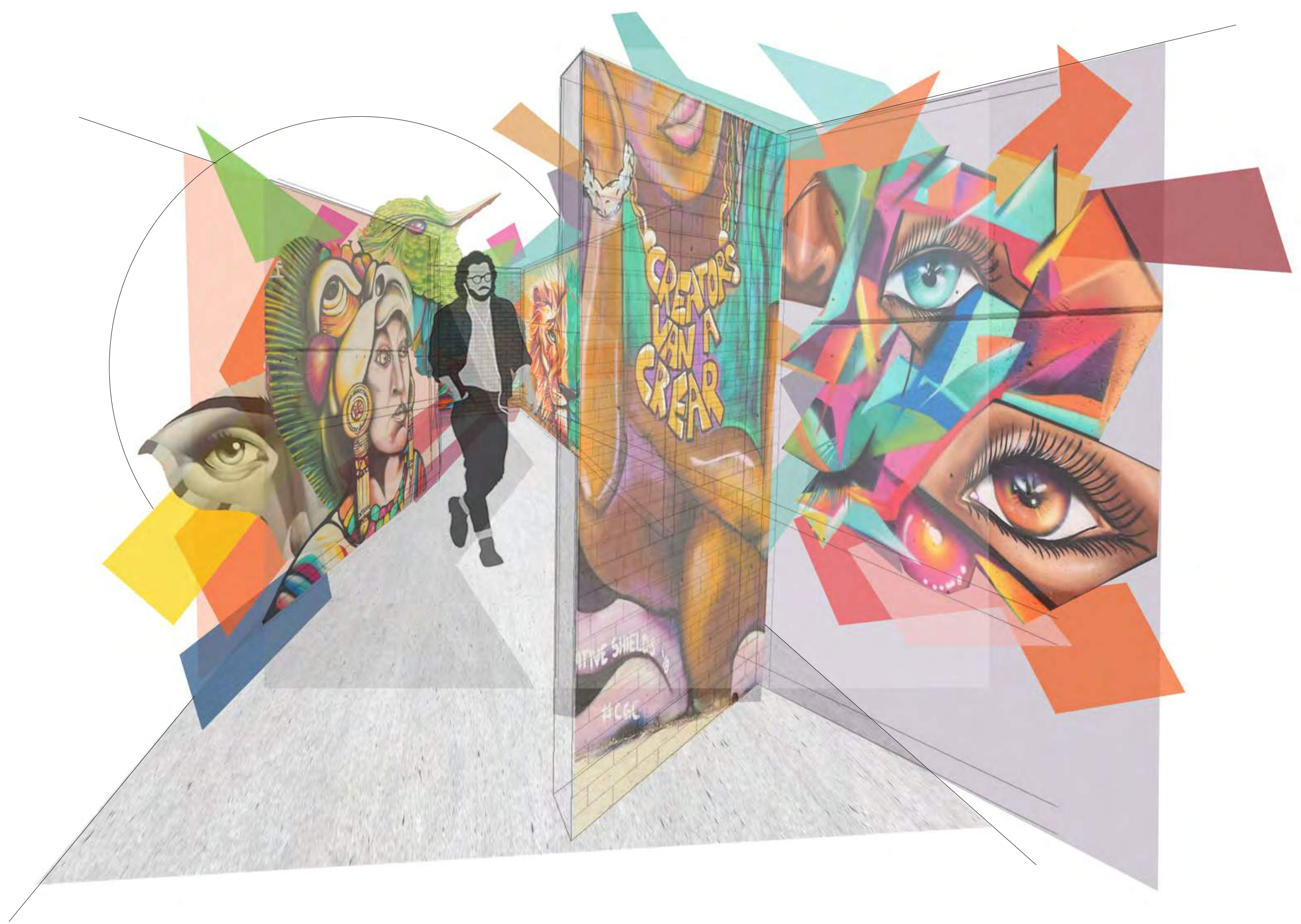




\section{Conclusions + Reflections}

Conclusiones + reflexiones

\section{DESIGN PROCESS}

The author's own interpretation of the mural work is entirely intuitive and diagrammatic. There are many other ways in which this art could be interpreted. This is the process which was discovered and followed during this design-led research.

\section{PROGRAMME}

This exploration was driven by the programme of supporting the community's move from the margins of the city right into mainstream culture people's path in the city centre. Creating a forced encounter inspired by ideas of tangible and intangible layering through the use of walls, memories and histories. For this community the walls has always been a canvas and this concept looked at how the wall as a canvas could create an urban gallery to enable the creation of art but also to showcase it.

Looking at how this could be further developed, usability and circulation could be further improved and scrutinised. Even though circulation was one of the key drivers in the development of this design, there is still room for improvement and further consideration into how people would actually move through this space. The idea that the architecture guides the user is something which was tested but could be further refined. The spatial qualities could also be strengthened through further exploration into aspects such as materiality, lighting and also the potential to fully enclose or open the space. This concept could also be broadened by creating multiple of these urban galleries and strategically placing them throughout the city in order to increase their impact but also the awareness and visibility of the community.
The key aims of this design were visibility, awareness and exposure. At a concept stage, this design is beginning to address all of these. It is creating a space which increases the visibility of this community and their art within the city, exposing this marginalised community to the public. The focus of this design was the art form of painting/graffiti which is one of the many forms of art this community produces. It created a gallery to display their memories and histories. 


\section{"Los jóvenes se apoderaron de las calles y dijeron 'No es la guerra'. Vamos a cambiar nuestras realidades." - Kbala}

"The young people took over the streets and said 'It's not war. We will change our realities." 


\section{AN INVITED ENCOUNTER}

CONCEPTO 2: ENCUENTRO INVITADO 


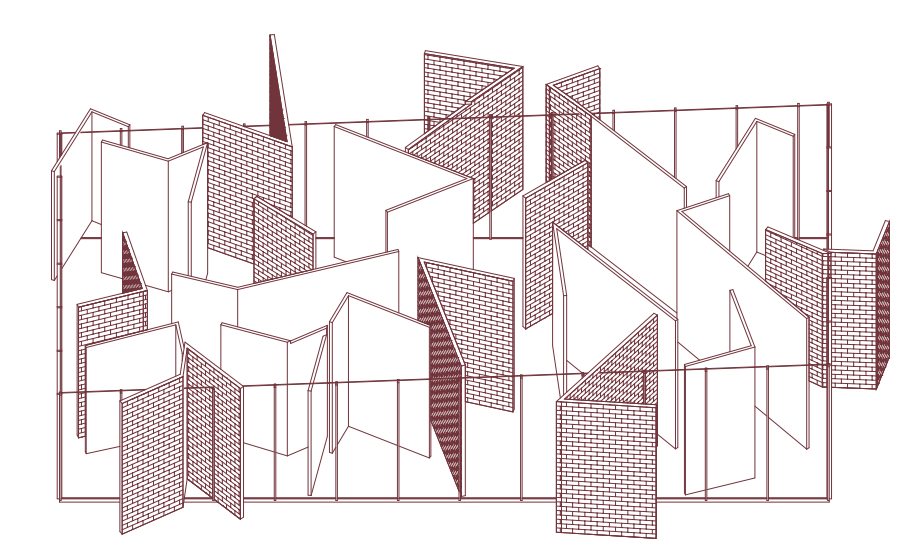




\section{Concept Two Introduction}

\section{Introducción al concepto dos}

This programme exploration stems from a desire to acknowledge all forms of artistic expression within La Comuna 13. Not only do the community members produce beautiful murals but also express themselves through the art of dance. There is a growing culture of hip-hop dancing within La Comuna 13 , which is a form of self-expression but also a form of income. With this in mind, it becomes important to develop a program which would enable both forms of artistic expression alongside each other.

The idea of invitation rather than direct provocation lead to the development of a programme of a dance pavilion in another part in the heart of the city. This is based on the intention of creating a space for the community to perform in a large urban space and to be seen by the wider public. The concept is driven by the intended set of interactions between public and dancers, where the public can engage with the architecture hence the 'invited' aspect. It would offer the public the opportunity to quite literally place themselves within this community space and their arts (both paintings and dance). It offers opportunity for interaction and reflection.

The development of this programme is still largely influenced by the previously mentioned ideas of layering and place-identity but it also introduces the new aspect of layering by merging the various forms of art being produced in this community into one space. The intentions of exposure are still largely present here as well through the selection of the site and the architecture itself, aiming to create a stage for this community to dance on.
The process begins with an inquiry into the relationship between dance and architecture which leads to a precedent study and a photographic study of a dancer. This is then used in conjunction with the shapes extracted from the art and some new diagrammatic interpretations to create the layout.

The design of the 'architectural shell' is a key part of the pavilion design placing importance to its openness. The site was carefully selected to be large enough in order to offer the members of wider mainstream society a distance in negotiating engagement with the architecture and its arts. But now also the architecture is placed to invite the public to interact and allow them to submerge into the arts as a way of interaction. 


\section{DANCE + ARCHITECTURE}

Danza + Arte

It has been argued that there is a direct link between dance and interior architecture. The programme of a dance studio usually brings to light not only the concerns of body in interior spaces generally, but considerations of physical movements in the making of its architecture more particularly. It also involves quite a significant consideration into other aspects such as materiality, atmosphere, the sensory and the perceived. It becomes essential to further dwell in the field of somatic-spatial considerations before engaging in the design process of the invited encounter.

Bloomer and Moore describe how our movements are ever subject to the same physical forces as are built forms and may be physically contained, limited, and directed by these forms (Bloomer, 1977, p. 57). Due to the interaction of body and movement with architecture being so critical and so constant, it requires close attention. There is a link created here with some of the ideas explored in the Forced Encounter with architecture becoming a guide for the body as it moves through space yet here it becomes more about an actual interaction between body and architecture.

The idea of movement is still hugely relevant, it just becomes less about moving through a space and more about moving within a space. Weinthal explains how the movement of a dancer is a short-sighted representation of how the body moves through space. It is choreographed and predictable, unlike the movement of a casual occupant who walks through a room and can make decisions about their trajectory based upon environmental cues, which makes their movement unpredictable (Weinthal, 2018, p. 217). This predictability of the dancer becomes a significant thing to consider in the design process. It means that there is a set of movements which need to be designed for. Their movement becomes one of the key drivers for the design of the interior architecture in terms of affordances and space allowances.

Weinthal takes an interesting approach to analysing the relationship between dance movement and drawing. This becomes relevant because one of the key processes used throughout this design-led research is both analogue and digital drawing. She discusses how point, line and plane can help to translate back and forth between one, two and three dimensions, where the point can be seen as the fundamental element upon which everything else is built (Weinthal, 2018, p. 217). Looking at dance through drawing is also explored by Rudolf Laban, an influential pioneer in graphic notation for dance. He describes movement in terms of the "frontal", "vertical" and "horizontal" planes which provides a triaxial structure. Looking at the vertical and horizontal dimensions becomes especially relevant to movement in architectural space (Bloomer, 1977, p. 58).

These ideas introduce some compelling concepts when it comes to drawing as they could begin to influence the creation of the drawings and the design process. It could become a layered system of designing where the drawing begins simply through dots, then develops to lines and eventually to planes. There is also a link created between Weinthal's and Laban's drawing theory and the ideas previously discussed when looking at "The Wall", such as the concept of planes and the vertical elements which interrupt and create space. The planes in the design of this Invited Encounter aim to divide space but also provide dancers with the necessary surfaces and materials to perform. It becomes more of an interactive element in the interior architecture rather than purely a means for exhibiting the art and guiding people through the architecture, as seen in the Forced Encounter.

When it comes to dancers interacting with interior architecture, Bloomer and Moore illustrate how dancers speak of "feeling" space. They explain how Marth Graham who was an American modern dancer, would regularly base a set of exercise on the haptic experience of space; her students were asked to hold, push, pull, and touch pieces of space and places in space. One of the outcomes of this type of dance training is that the entire body gradually becomes mobilised to touch and feel space, so that movement becomes not a vague indescribable set of reflex actions, but an articulately felt interaction with the positive stuff of space. The dancer and the space animate one another as partners (Bloomer, 1977, p. 58). The notion that the interior architecture and the dancer can not only interact but also somehow 
"animate" each other is intriguing. The interaction allows the dancer to further their art while it also makes the wall an essential part of it. This idea of haptic experiences is further explored by Bloomer and Moore as they explain how the fit and movement of our bodies within and around buildings are significantly affected by our haptic sense, by the tactile qualities of the surfaces we encounter. Smooth surfaces invite close contact, while rough materials generate movemont in a wide radii. Changes of texture can often signal events and it would almost be possible to generate a whole choreography of movement through a space from the textural changes alone (Bloomer, 1977, p. 71). These ideas become heavily influential on the design process as they begin to inform materiality and surfaces.

"All architecture functions as a potential stimulus for movement, real or imagined. A building is an incitement to action, a stage for movement and interaction. It is one partner on the dialogue with the body" (Bloomer, 1977, p. 59). One of the key aims of the pavilion in this Invited Encounter is to expose the wider community to the art being created in La Comuna 13 and this applies to all forms of art, not just graffiti. This pavilion creates a stage for movement for this community to practice and perform their dance while being seen and apprecited by the public. After looking into this literature, a precedent study is undertaken.

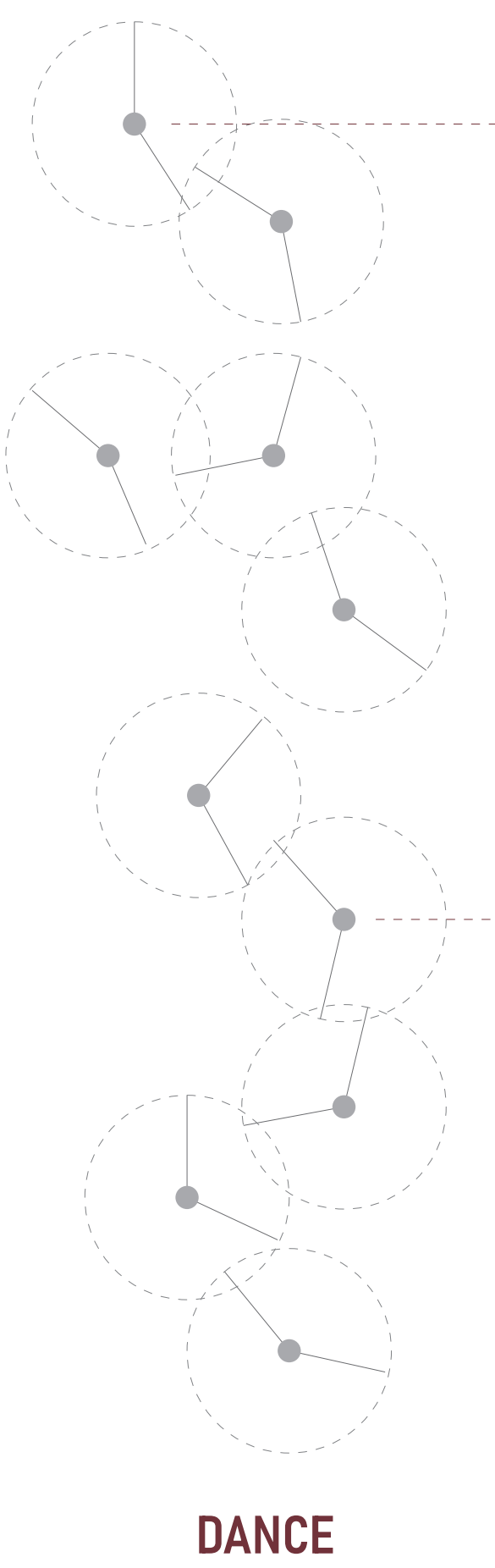

83 


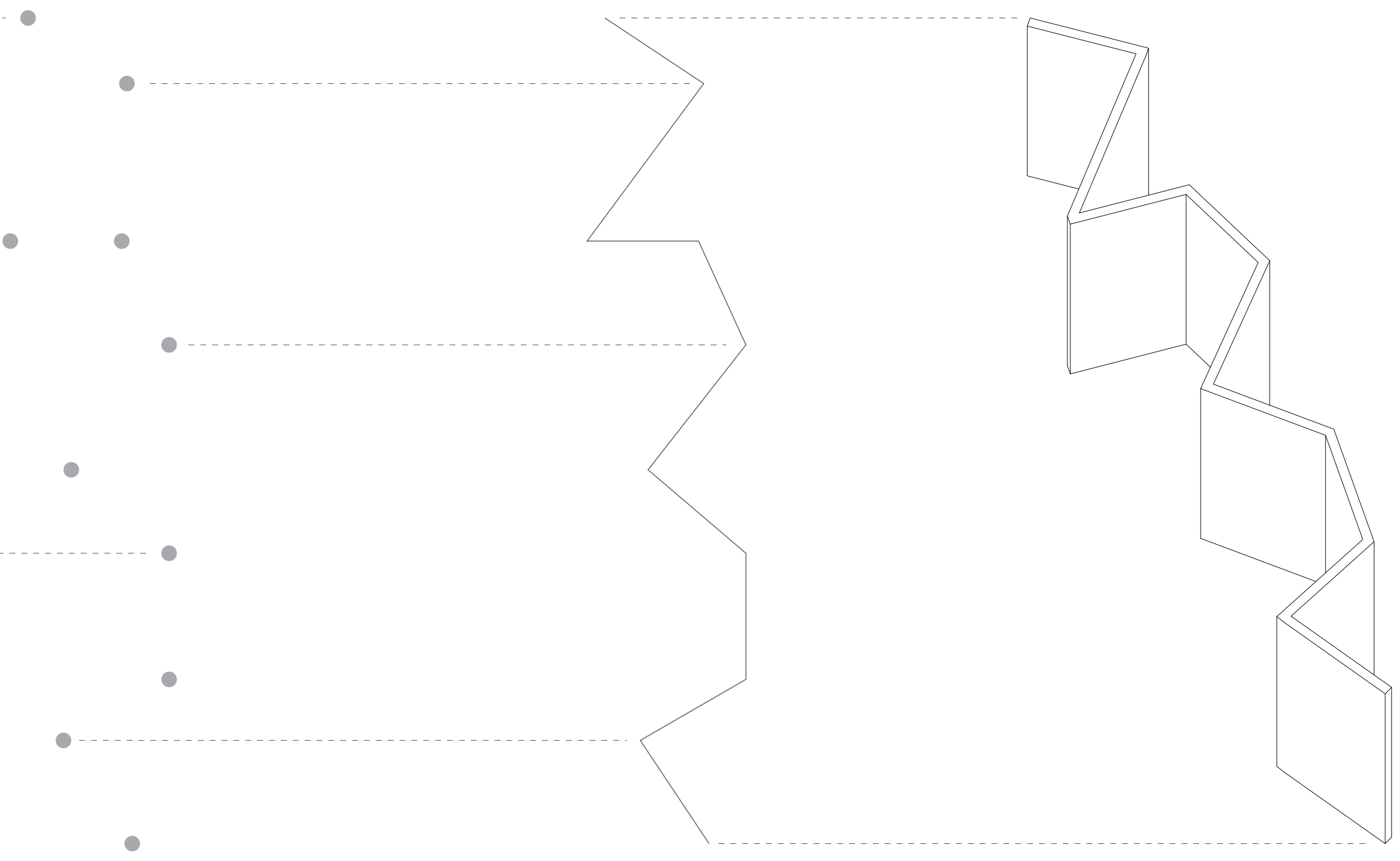

POINTS

LINES

PLANES

84 


\section{Museum Langen Foundation by Tadao Ando}

Designed by Japanese architect Tadao Ando, the Museum Langen Foundation is located in the countryside of the small town of Neuss, Germany. The Langen Foundation is located at the Raketenstation Hombroich, a former NATO base. The building consists of a double-skin volume, with a structure that is mainly made up of reinforced concrete, glass and steel. A glass envelope, supported by steel girders, protects the perimeter around the concrete core. This building is a constructed place that is not only an envelope for art but also exhibits itself.

This becomes one of the key inspirations for the design of the Invited Encounter. Firstly, having a completely glazed exterior which completely exposes the interior. This creates a stage for the dancers where everyone who walks past the pavilion can see them dancing or practising. It exposes their art form to the wider community whilst still encasing them. The walls within the pavilion will very likely consist of materials such as brick, wood and mirrored glass.
This content is unavailable.

Please consult figure list for further details.
This content is unavailable.

Please consult figure list for further details. 


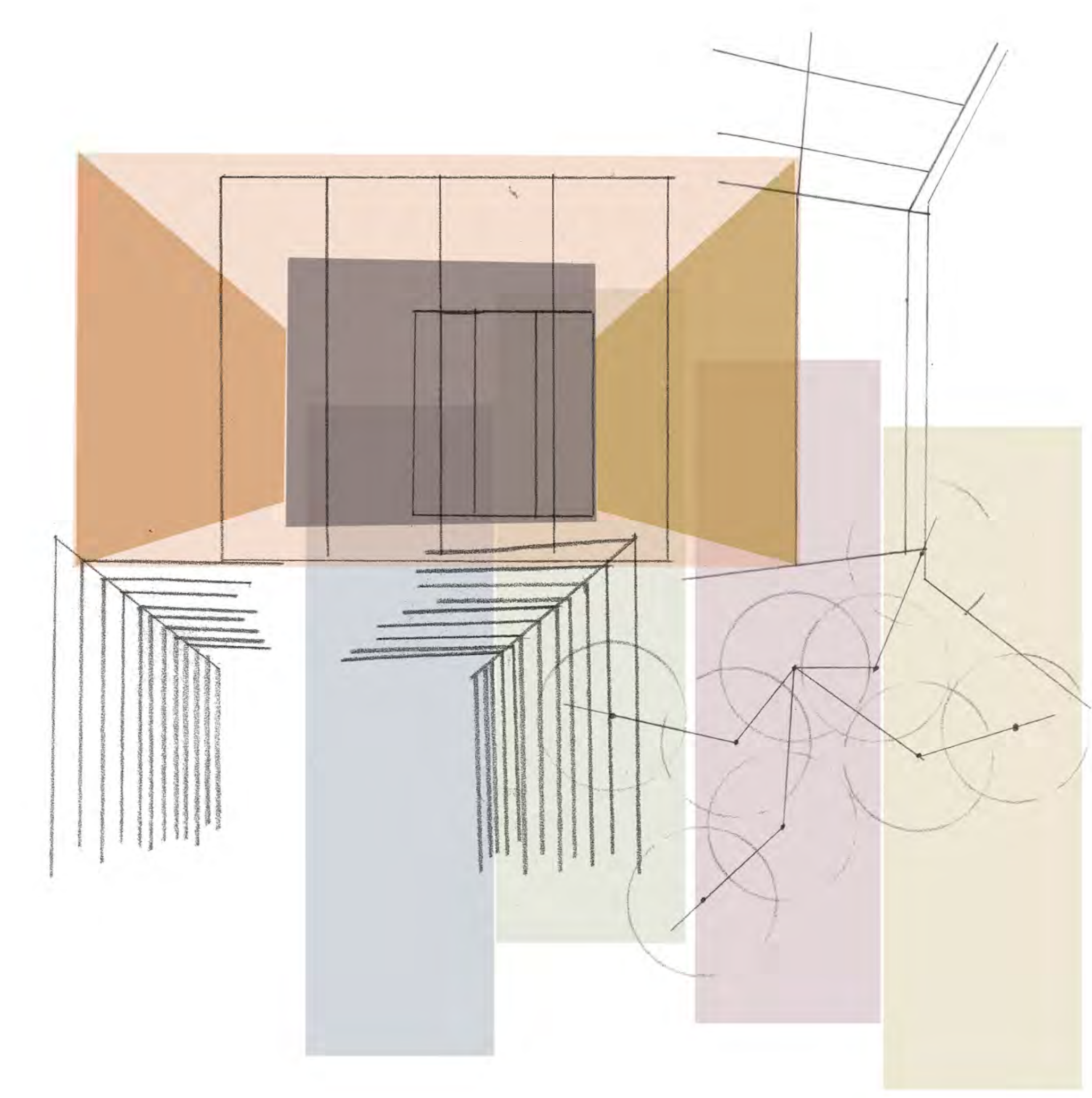


This content is unavailable.

Please consult figure list for further details.

Figure 36

\section{Joan Weill Centre for Dance by Iu + Bibliowicz Architects}

This building is a home for Alvin Ailey American Dance Theatre and is located in midtown Manhattan, New York. Projecting from the hefty L-shaped core are layers of glass-walled dance studios, which appear to float without obvious means of support, just like Ailey dancers in performance. Each of the 12 studios has slightly different proportions, with ceiling heights ranging from 4.8 to 6 meters. All but one has sprung floors of yellow Southern pine mounted on rubber supports. The architects have explained how solidity and transparency were powerful generators of form. And also how, for them, this building is not about displaying their creativity but it's a stage for the dancers to display theirs (Bernstein, 2005, p. 292).
This building becomes a perfect example of architecture becoming a stage for artistic expression. By creating a façade that is entirely glass and exposing the dancer, their art can be acknowledged by the wider community. This is also a good source of inspiration in terms of materiality and surfaces as they have been designed specifically for dance within this space.
This content is unavailable.

Please consult figure list for further details. 


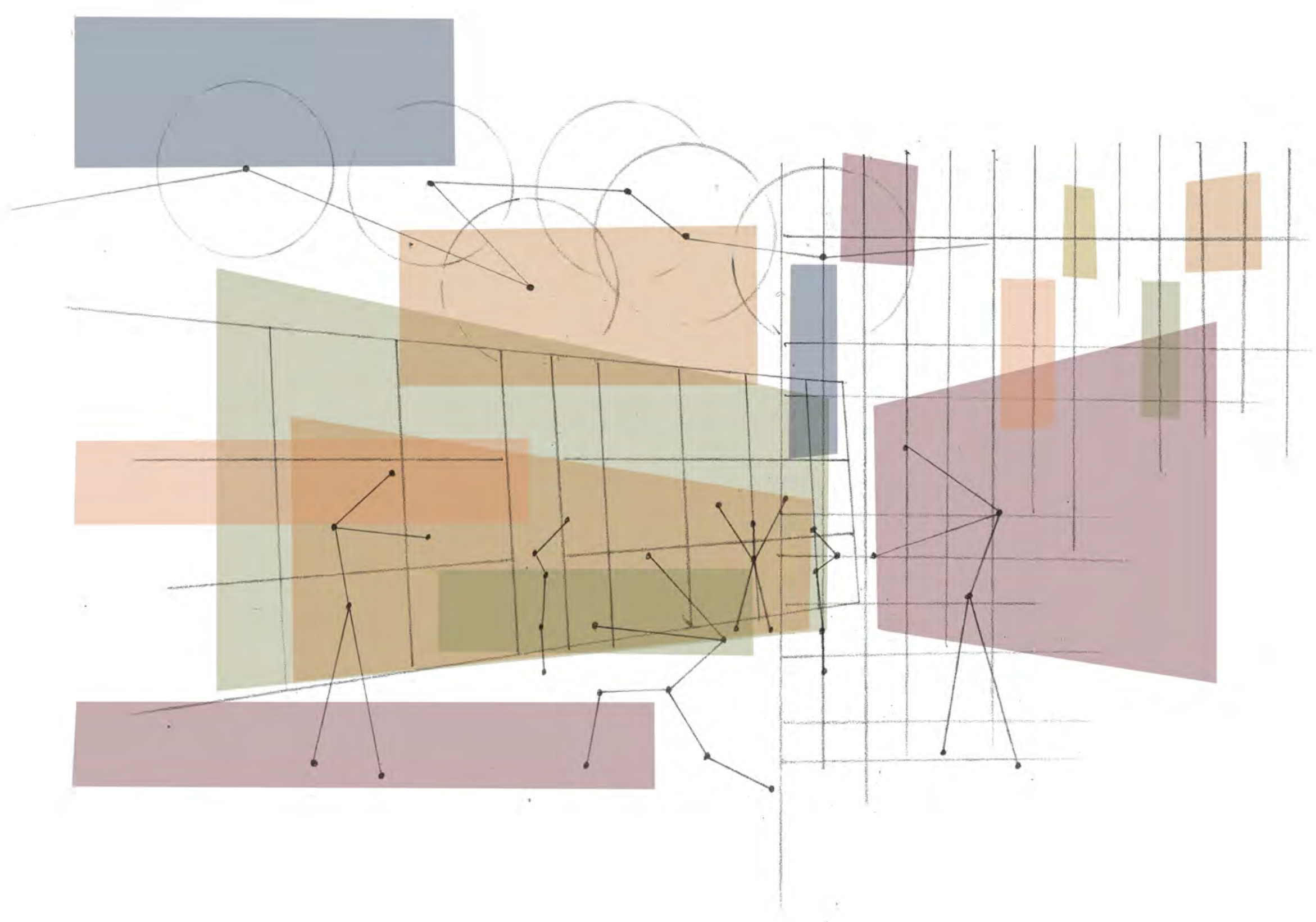




\section{Y Ballet School by $y+M$}

This is a classic ballet school located in a residential area in Tokushima, Japan. The entire second floor is the ballet studio which the architects did on purpose for the space to be as open as possible. They considered things such as the height of the ceiling to allow for all necessary movement such as lifts. The long hallway which leads into the ballet studio was purposefully designed to create a differentiation between ordinary life and the classic ballet school. The glazed external façade allows not only the community but other dancers to easily watch the classes or dances in progress.

This becomes relevant not only because it highlights the key features that are important to consider when designing an environment for dance, but also because this again creates a link to the ideas of essentially creating a stage. By allowing the community and other dancers to watch the dancing in the studio, it becomes an act of showcasing the art and exposing it to the outside world. It is a practice which is happening within but is at the same time displayed on the outside.
This content is unavailable.

Please consult figure list for further details.
This content is unavailable.

Please consult figure list for further details. 

With the background research into the connection between dance and architecture now established and supporting design precedents studies the design process now calls for a direct exploration of movements, space and materiality by observing the performance of a dancer. The chosen method for the development of this direct understanding was a photographic study of a professional hip-hop dancer; considering that hip-hop is La Comuna 13 members' main dance form.

The study is significant in not only providing new information, but it also confirms and reiterates some of the findings in the background research and the precedents. One the most significant findings is the importance of a space being designed for the practice of dance. This is something which is discussed in both the literature and the precedent study in terms of materiality. Whilst taking the photos, it quickly became evident that the dancer was finding it slightly difficult to dance on the floor of the photo studio as it was not wooden or entirely smooth. Watching this unfold highlighted the importance of materiality considerations in the design. In watching him struggle, there was also a realisation of the constant relationship between body and architecture which is also discussed in the literature. While dancing, the dancer is almost constantly in contact with the architecture whether it be through his feet, hands or other body parts. There is a continuous relationship and almost partnership between dancer and architecture.
It was interesting to observe that the interaction between dancer and architecture was not as expected. It is not always the literal tactile interaction of touching the floor or the wall but also being guided and confined by the architecture and creating an interaction in that way. Something which was observed was that the size of the space and the proximity to walls did not hinder the dancing, if anything, it encouraged further creativity and interaction. It was evident that there was a thought process from the dancer on how to simply make the most of the space he was given. This is important moving forward into the design process as this will influence the location of the walls within the pavilion. This concept introduces the idea that there is no ideal space allowance when it comes to hip-hop, as these dancers will just take whatever space they are given and work with it.

This leads to a further realisation that the hip-hop dancer in the following photographs was provided with a large, white space which was reasonably comfortable to dance on. The dancers in La Comuna 13 dance on the streets and have grown up learning to dance on the streets and take advantage of any space they find. This could mean that the dancers from La Comuna 13 might not have any particular requirements in terms of space to dance in but it could also mean that there will be a significant appreciation for spaces which vary in size and proximity as it allows them to decide the space they want to use. 


$$
x
$$



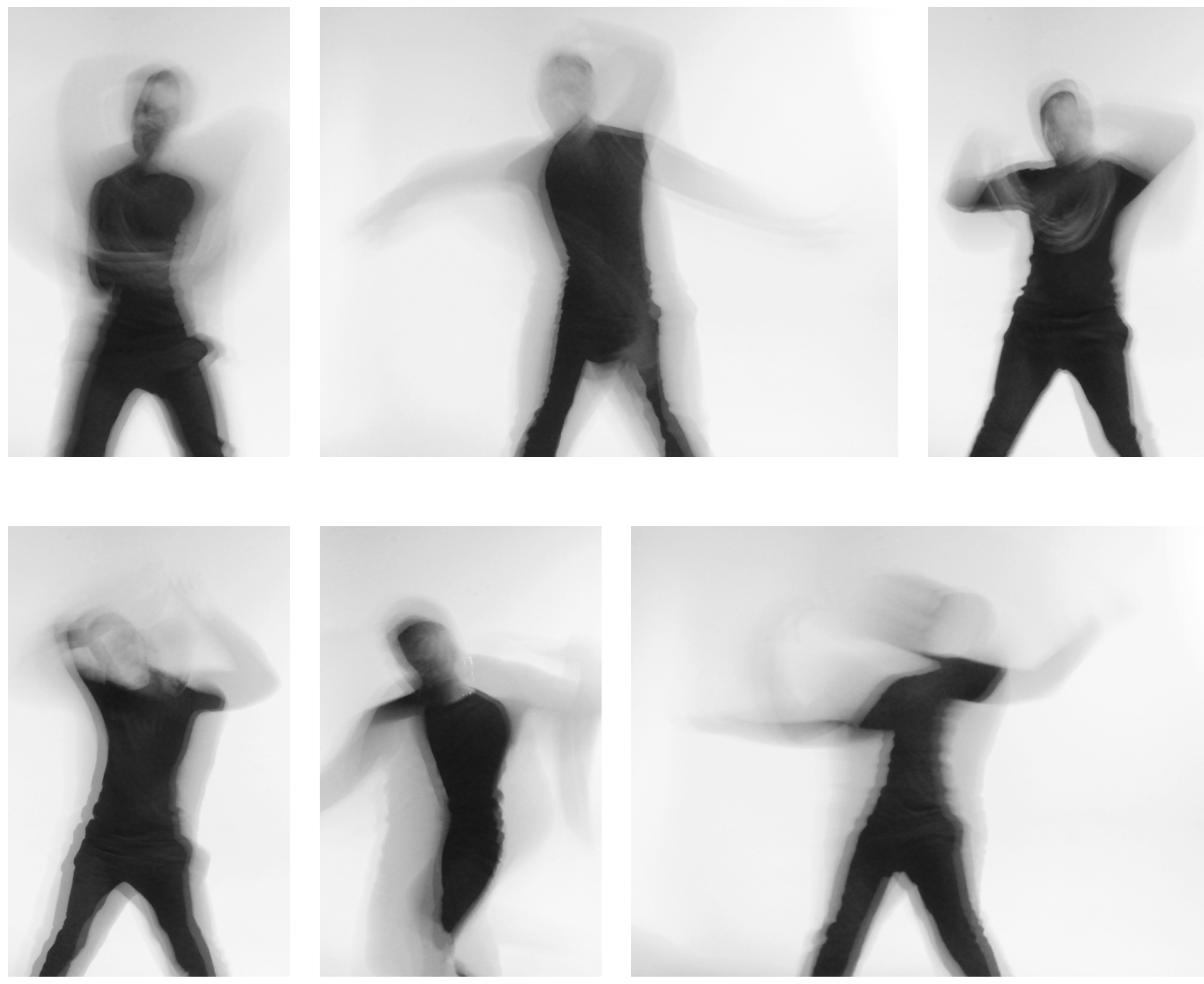

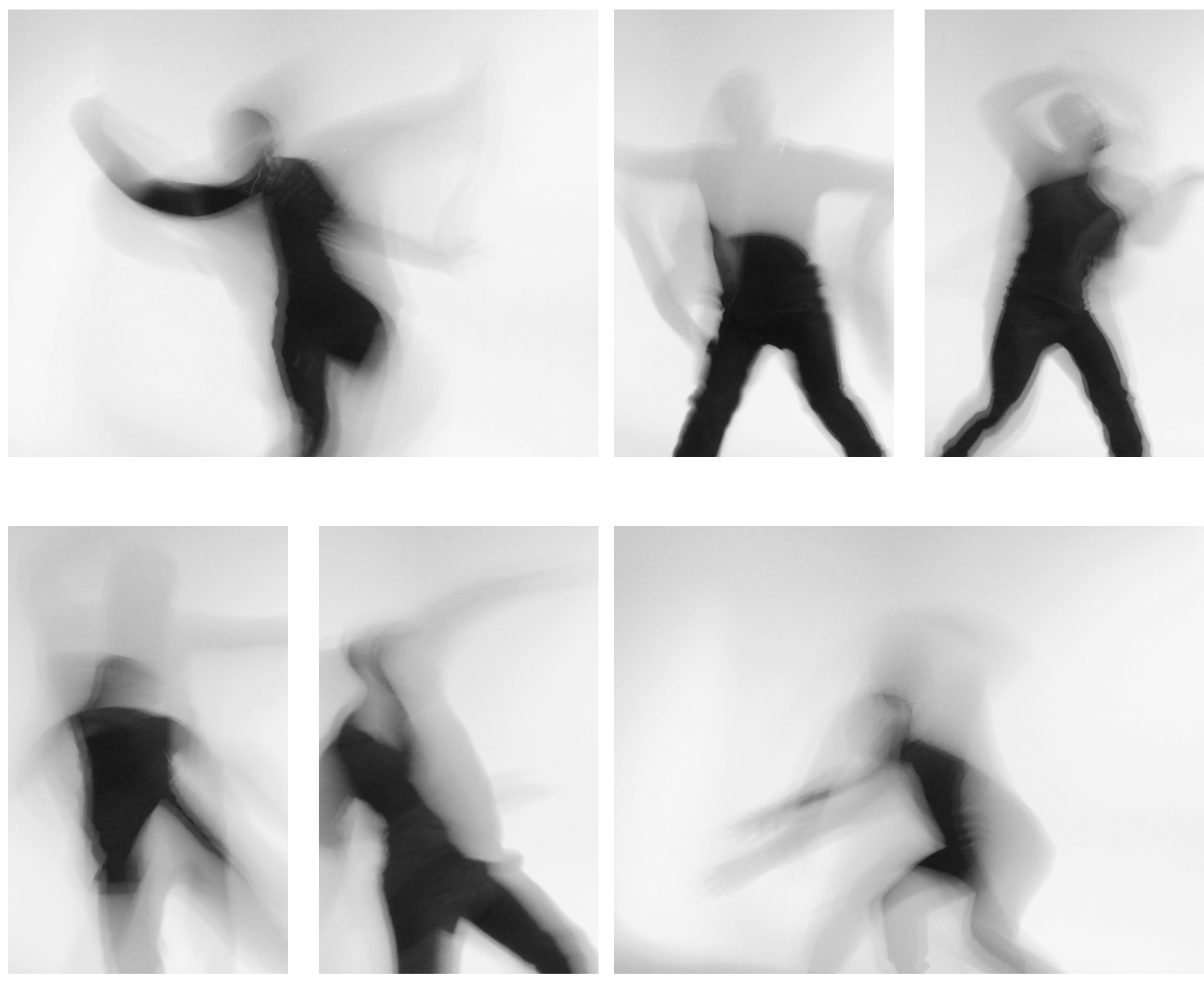

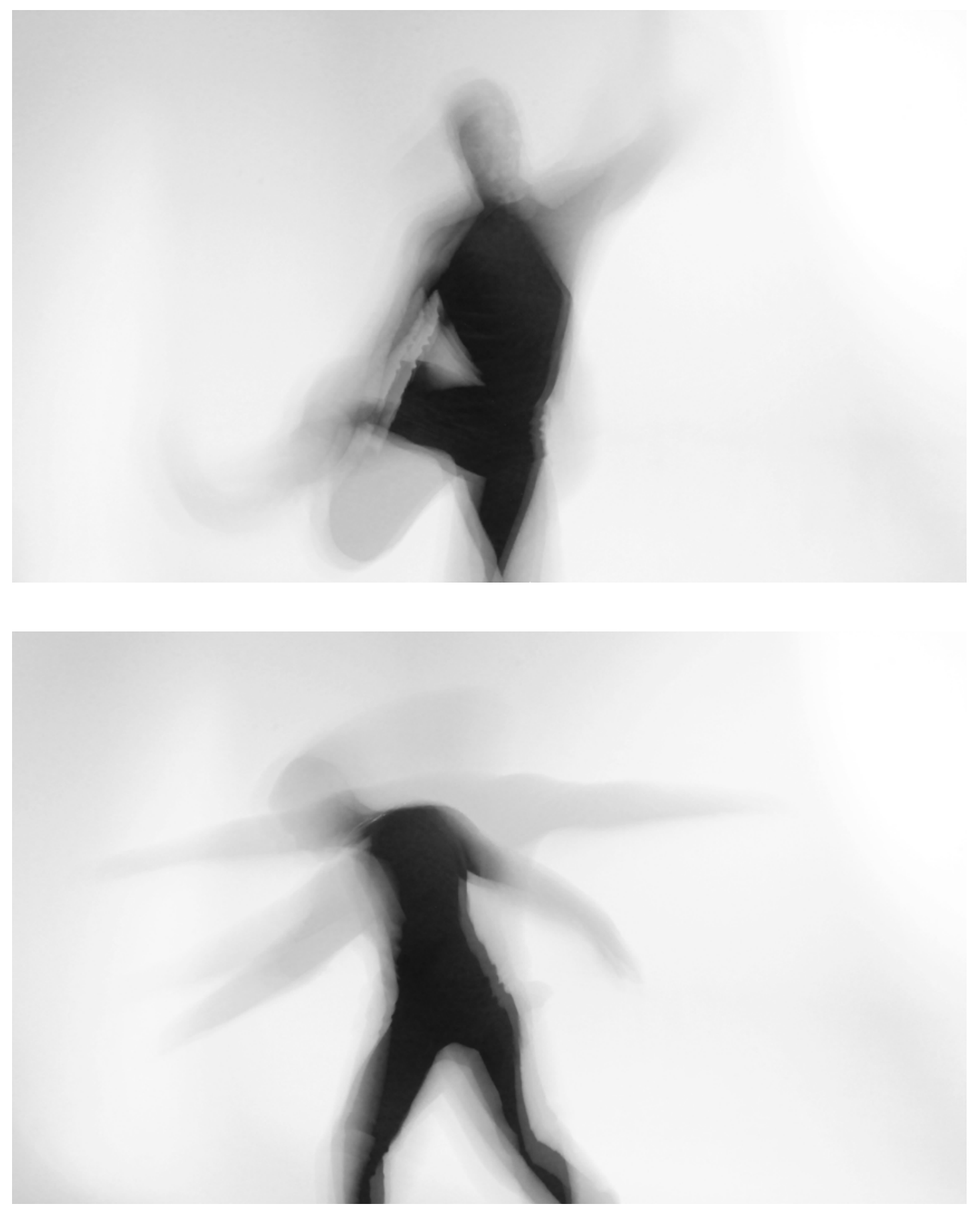

This photographic study creates a series of traces of movement which become a point of reference for the space required by a hip-hop dancer to carry out various dance moves. In the photographs, it is clear where the dancer's body has been and where it is at the time the photo was taken. This creates an archive of movements in which we can see that some dance moves require more space than others and therefore the space which is being designed should allow for both. 


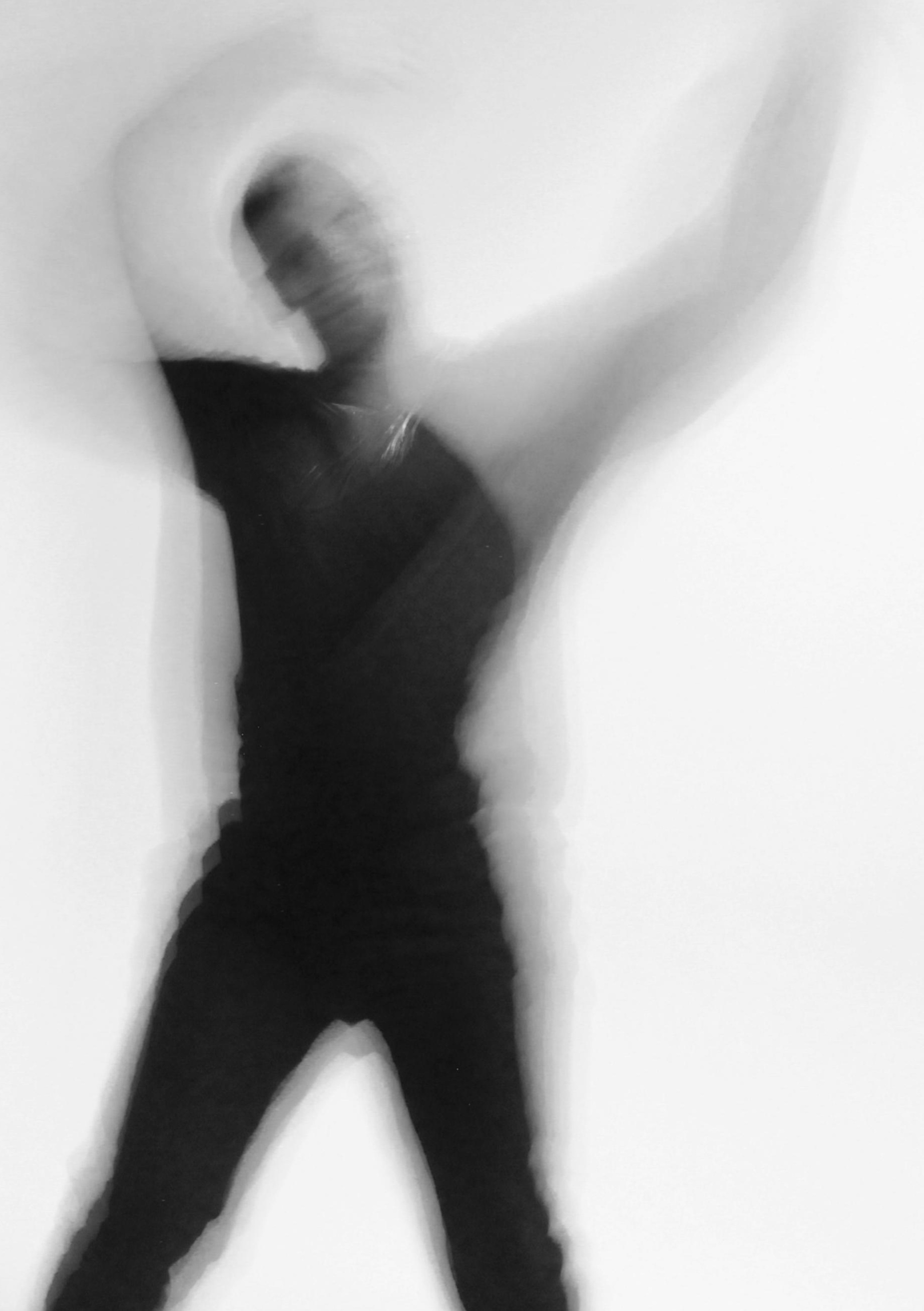




\section{The photo analysis process:}

This process simply involves an intuitive exploration and interpretation of the images from the photo study. Looking at shapes, angles, lines and points. This is informed by the ideas established previously in the literature about the relationship between architecture and dance where movement is described in terms of vertical, horizontal, and frontal planes.

This layered process starts to create a drawing which could become an influential tool in the location and spacing of the wall within the dance pavilion. The drawing could represent distances required for movement on a planar scale rather than a three-dimensional one.
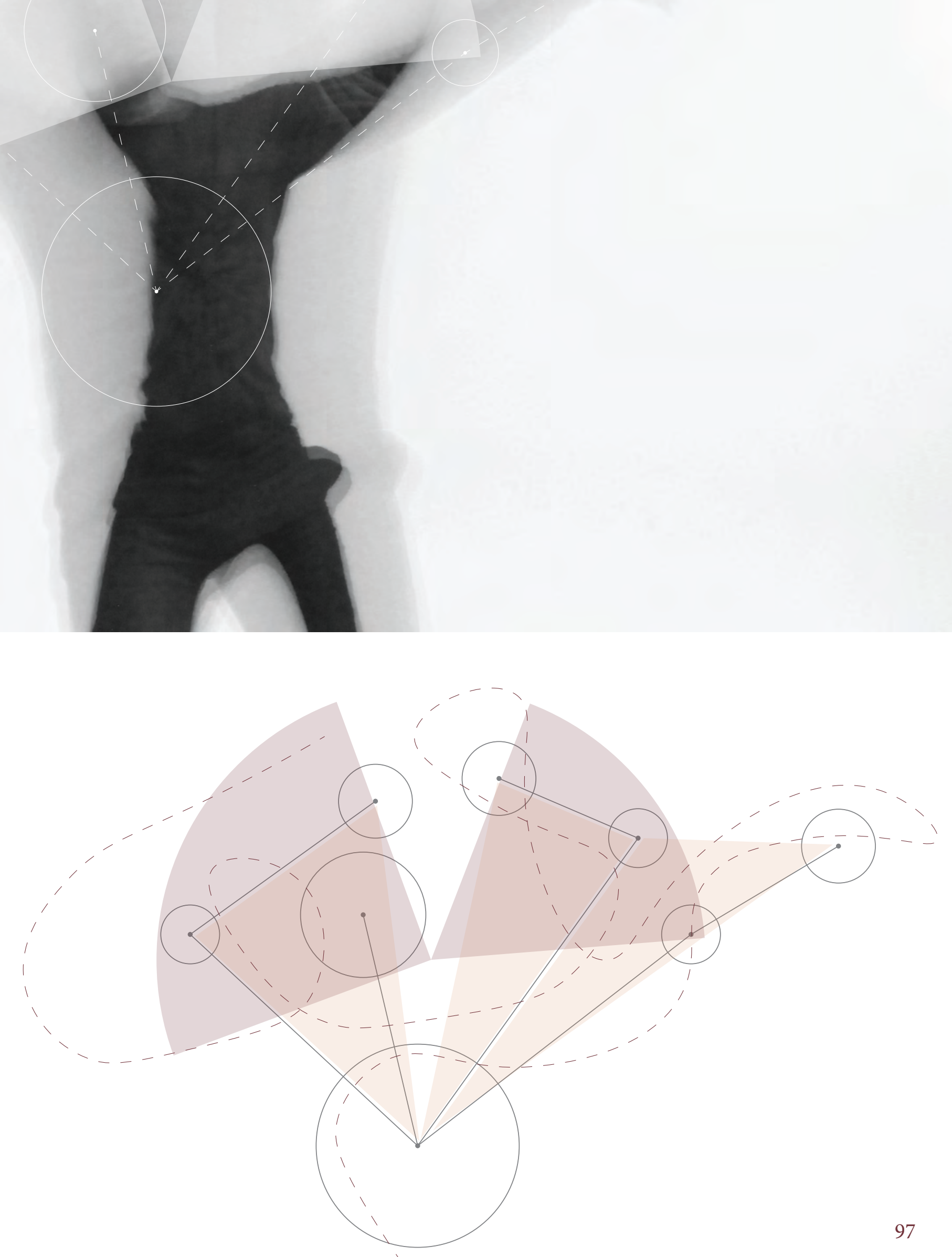

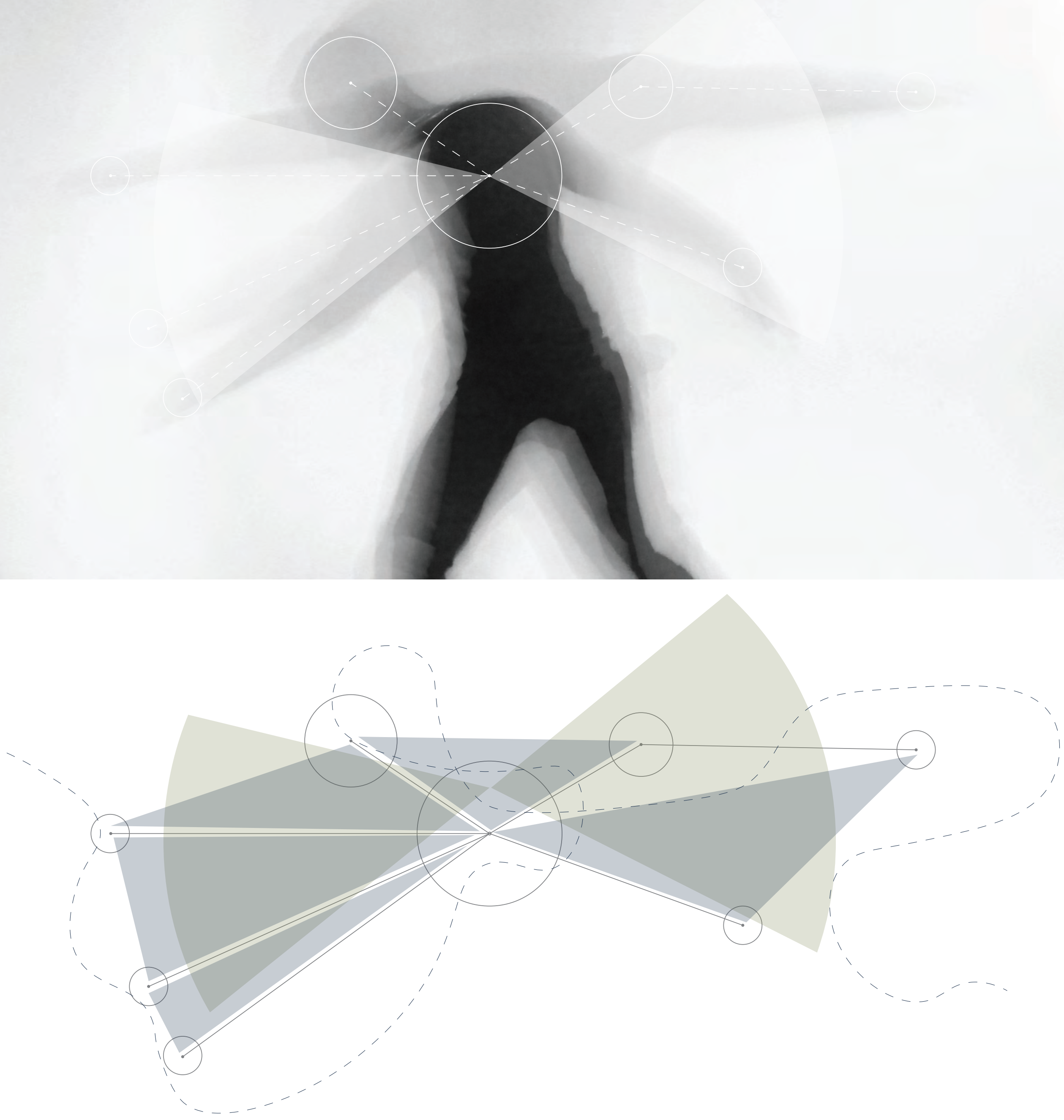


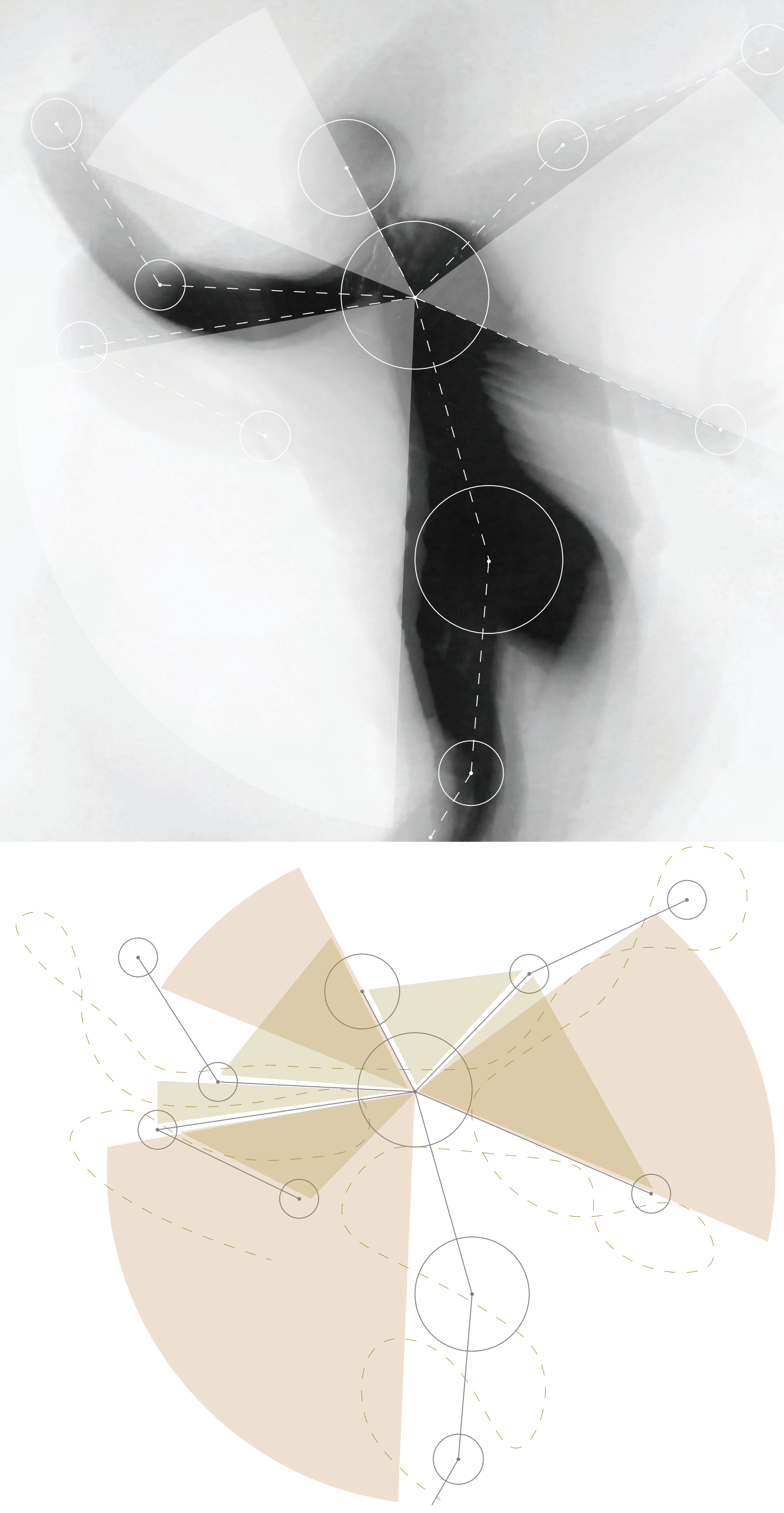




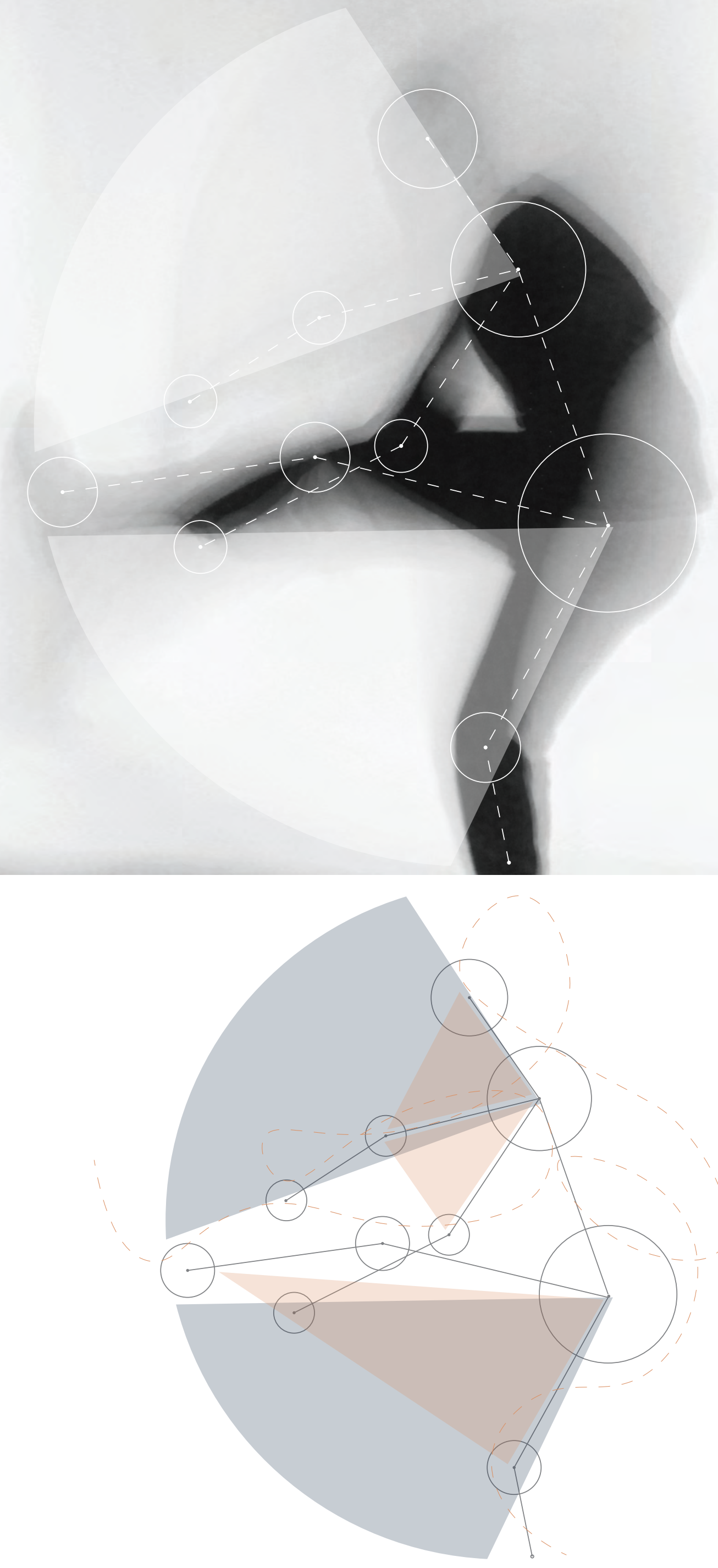




\section{THE DESIGN PROCESS}

\section{STAGE 1}

01

THE EXTRUSION

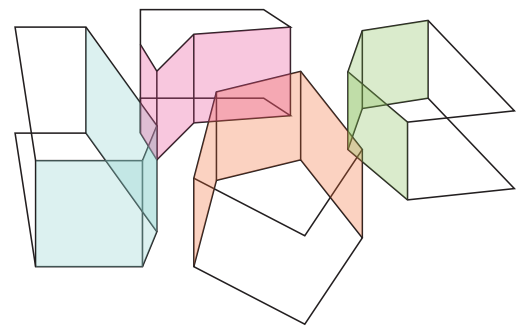

02

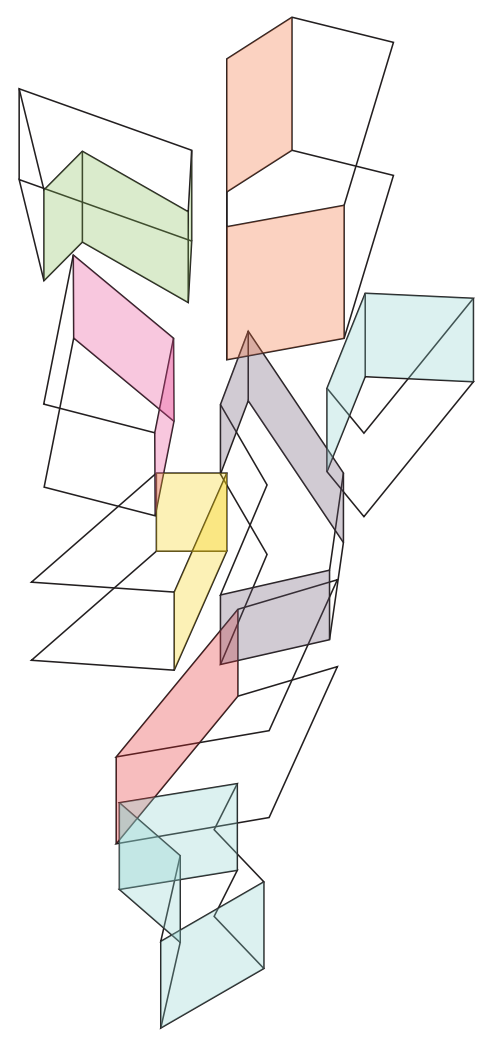

THE WALLS
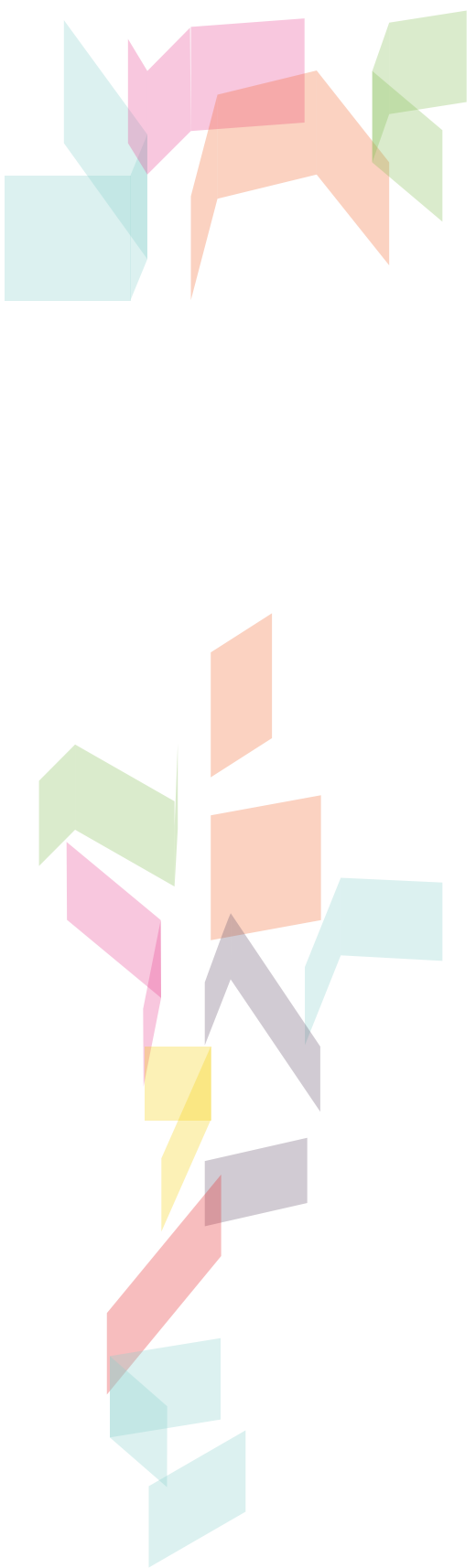

THE PLAN
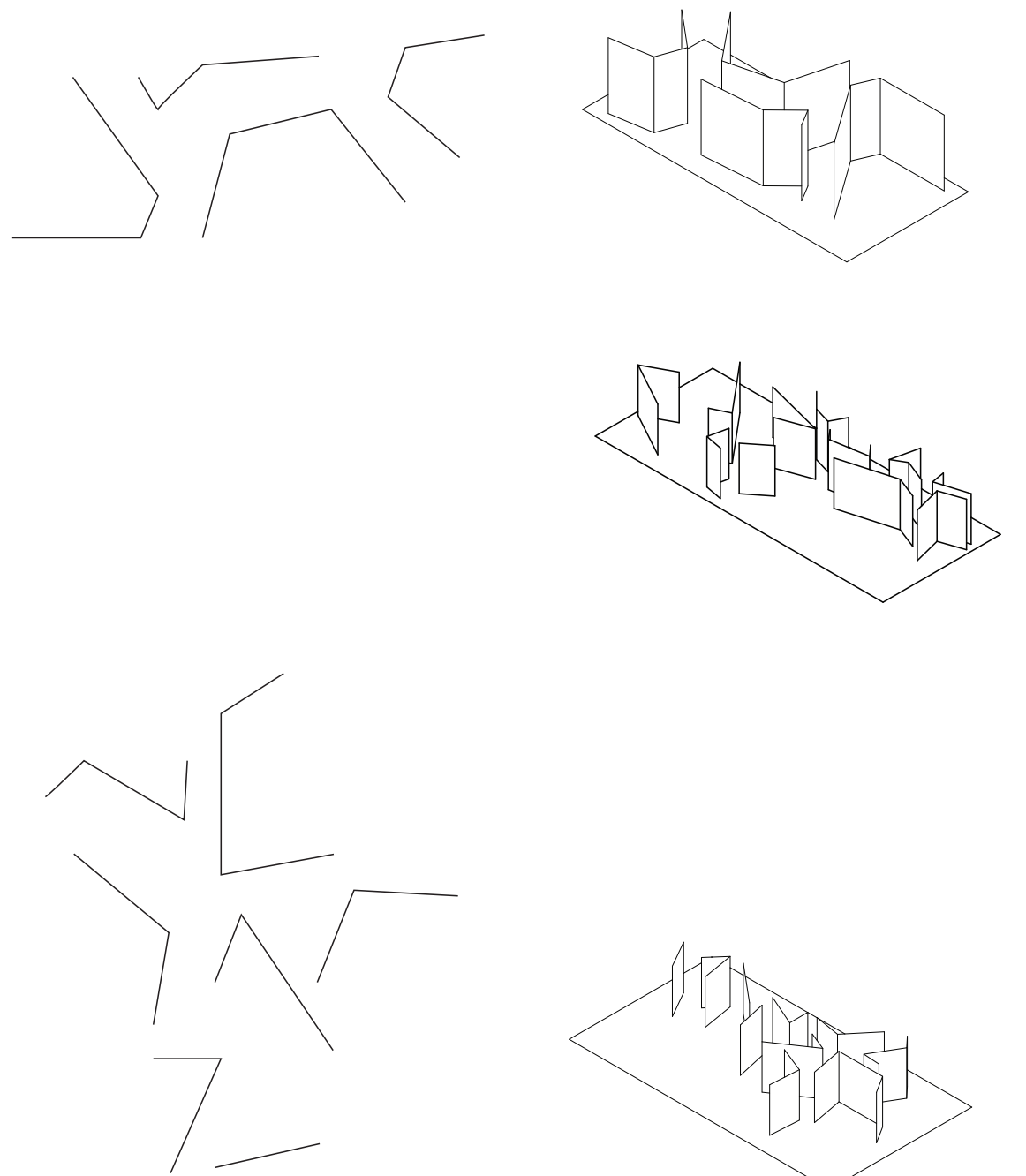

THE WALLS
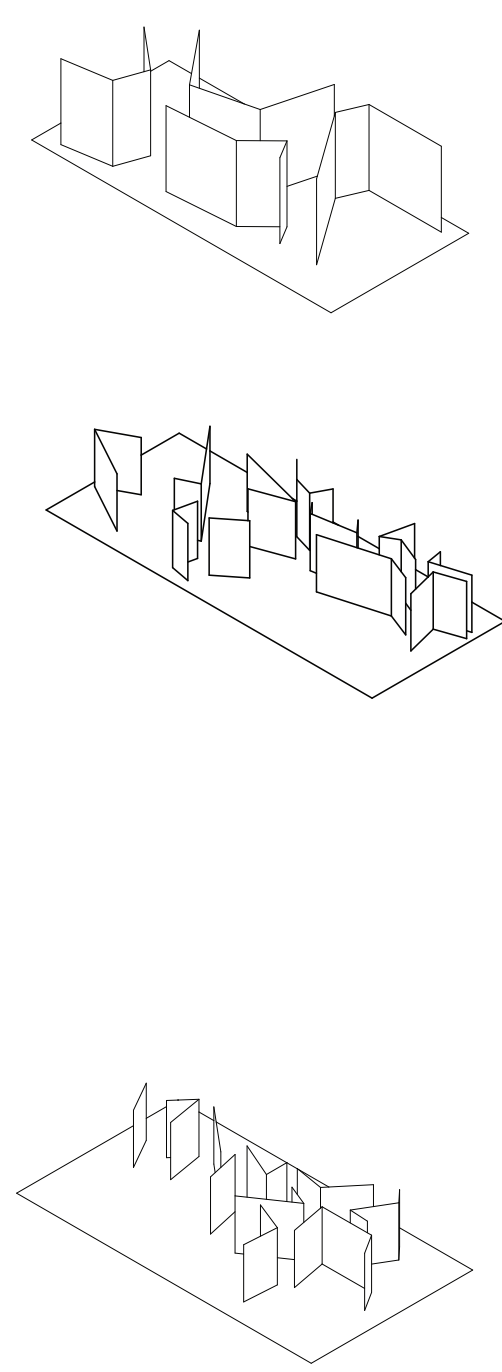
03

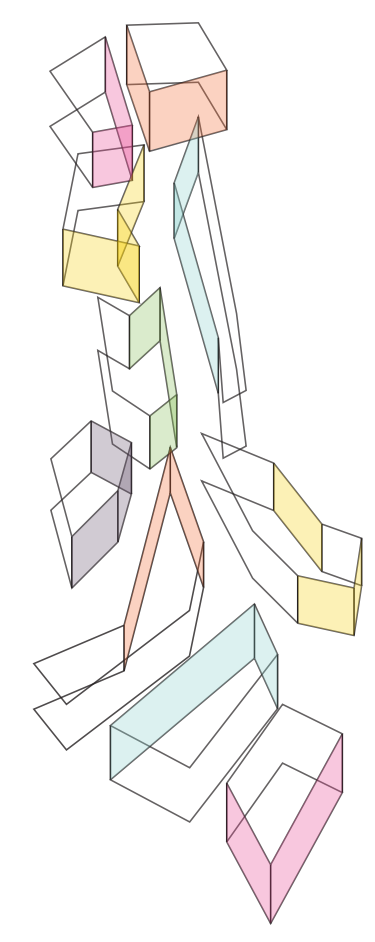

04

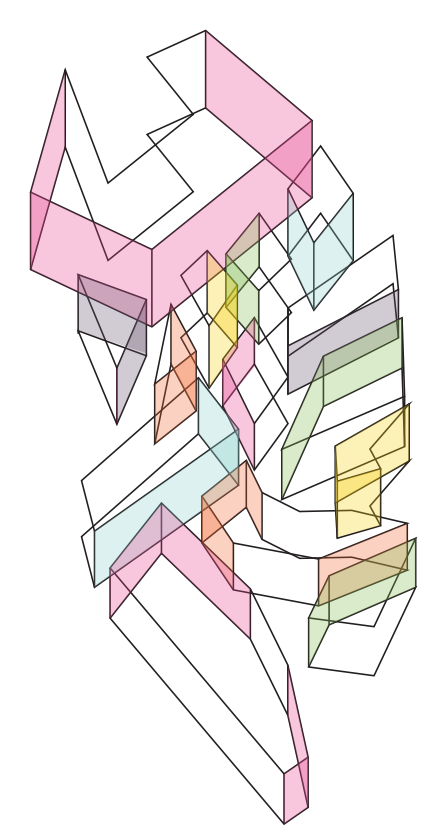

4

$3_{11}^{12}$

1

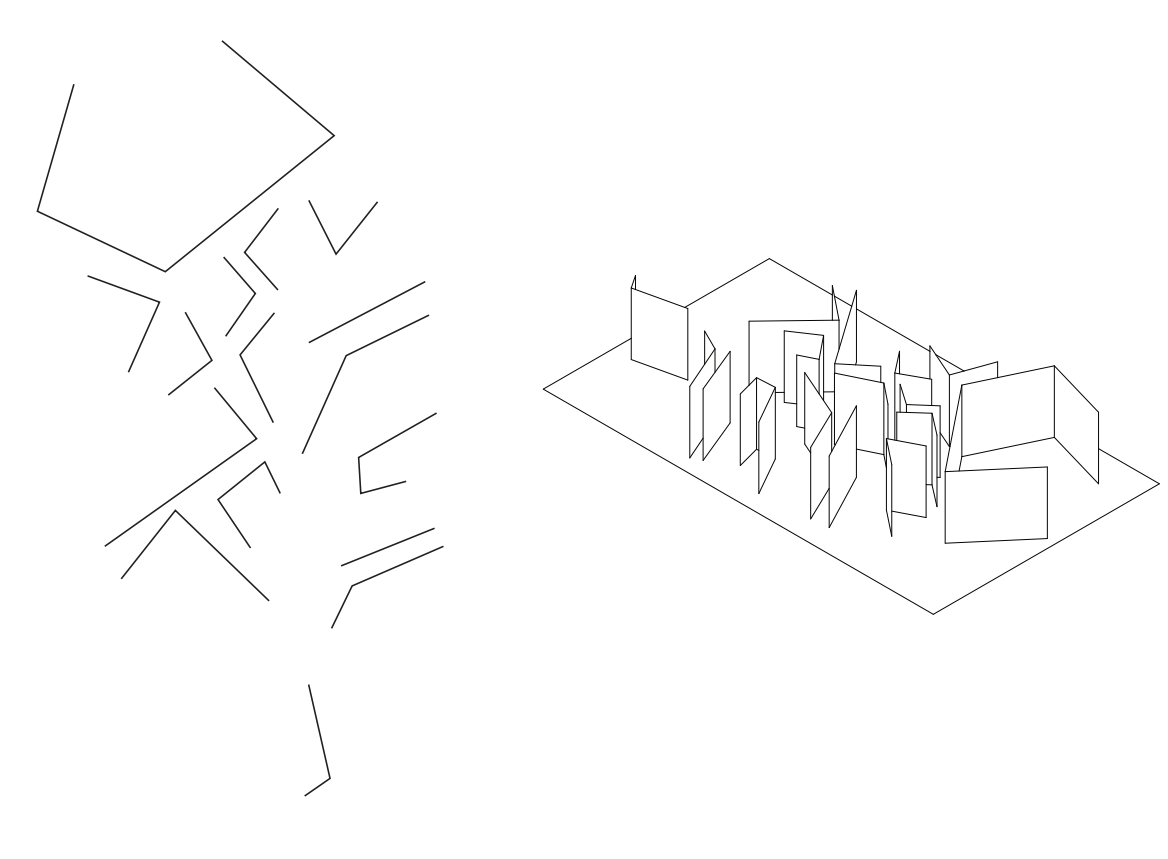

$$
\sum_{\text {Floor plan - Iteration } 1}^{1>>>1}<>
$$

102 
STAGE 2

\section{THE DEVELOPMENT}
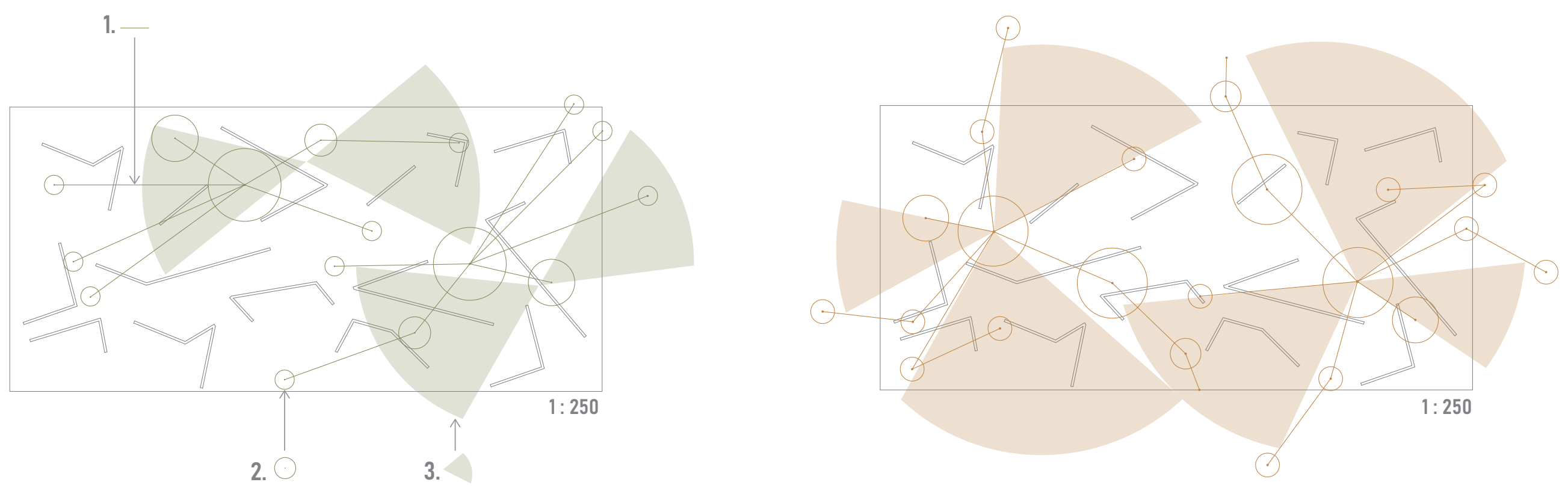

1. The lines are used to establish pathways

2. The circles are used to establish the areas in which the dance would take place

3. The coloured sections are used to establish the areas where exposure to the outside must take place
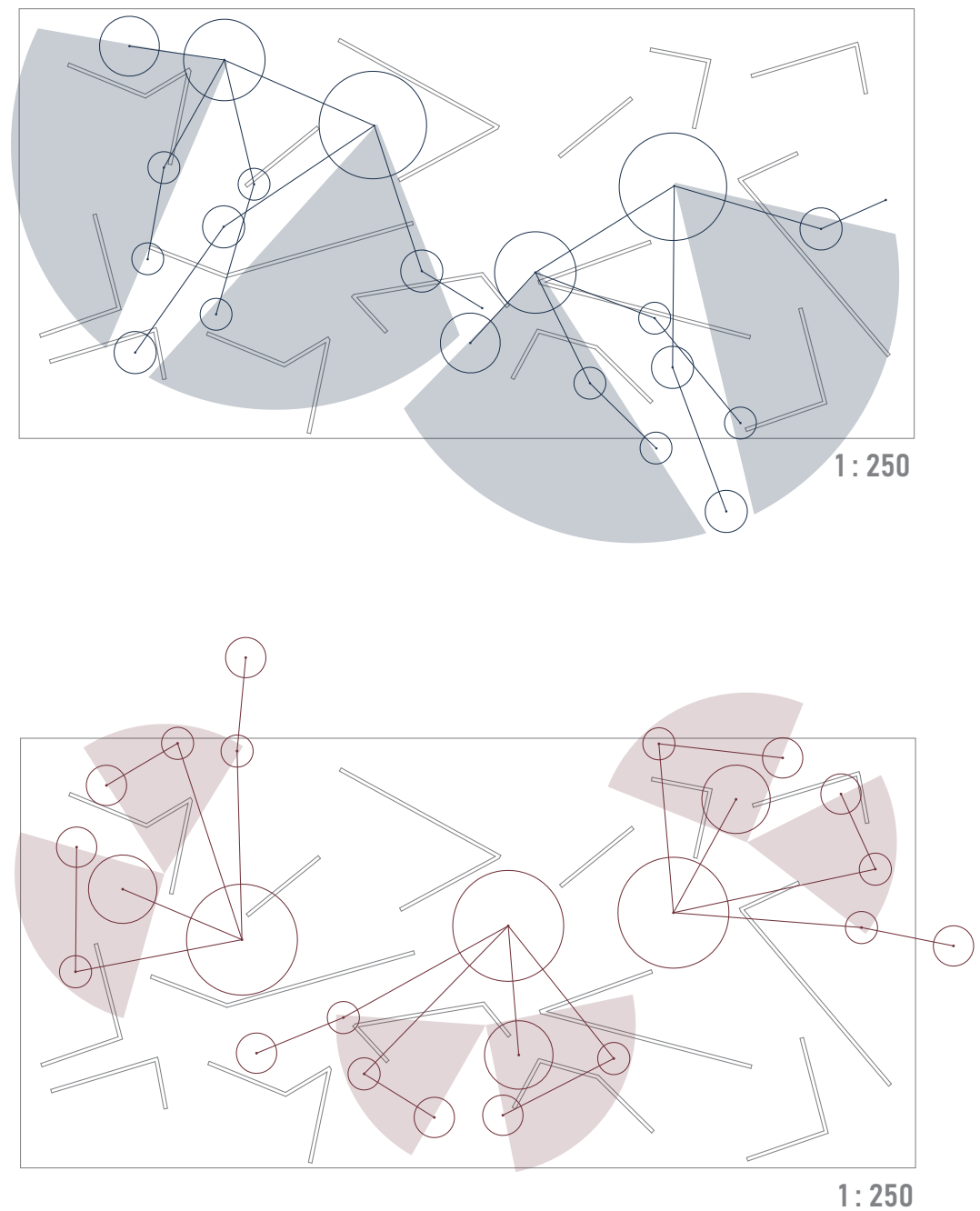


\section{THE RE-ARRANGING}
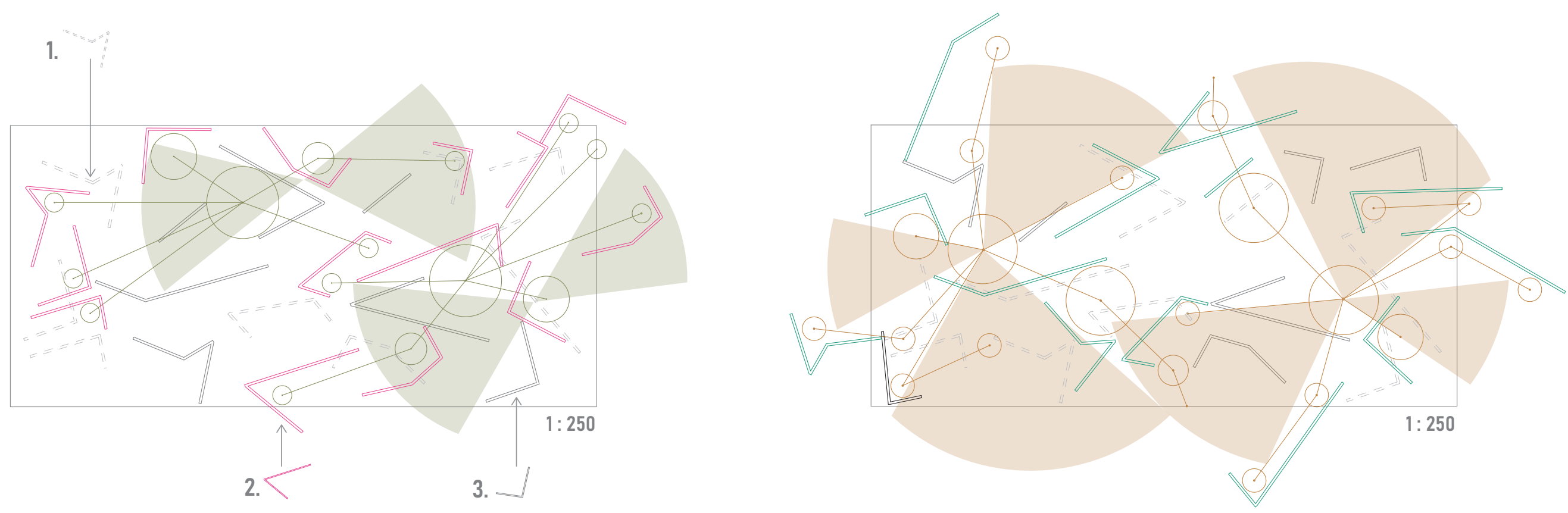

1. These are the walls which were moved or altered according to the photo study drawing guidelines.

2. The colourful walls are the new walls which were either moved from a previous location or simply introduced to the space following the new guidelines

3. The grey walls are the walls which remained unaffected
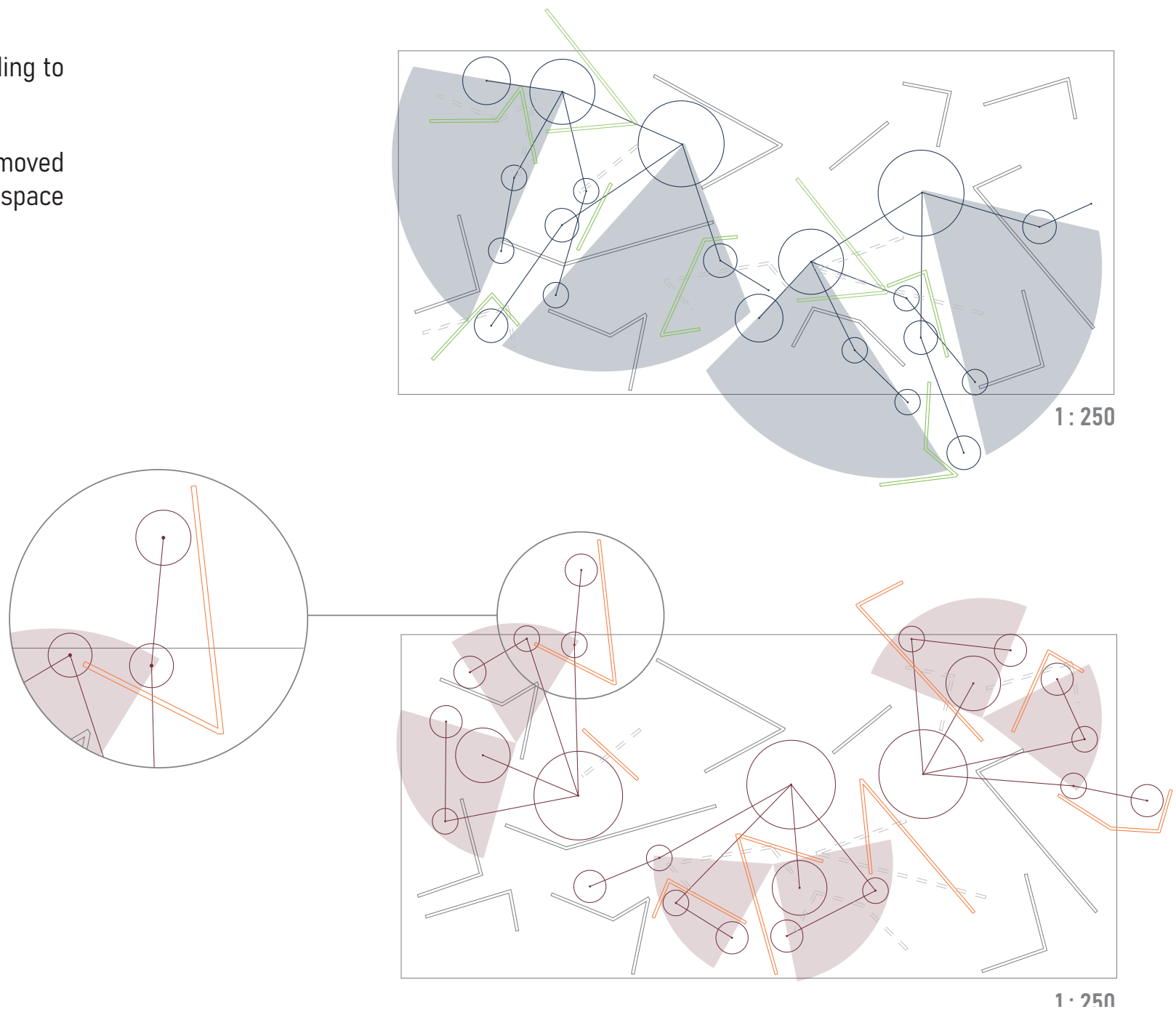


\section{THE DEVELOPED FLOOR PLAN}

One floor plan from the previous iterations is selected and analysed in terms of transparency and circulation. Then this floor plan is used to begin conceptualising the exterior shell.
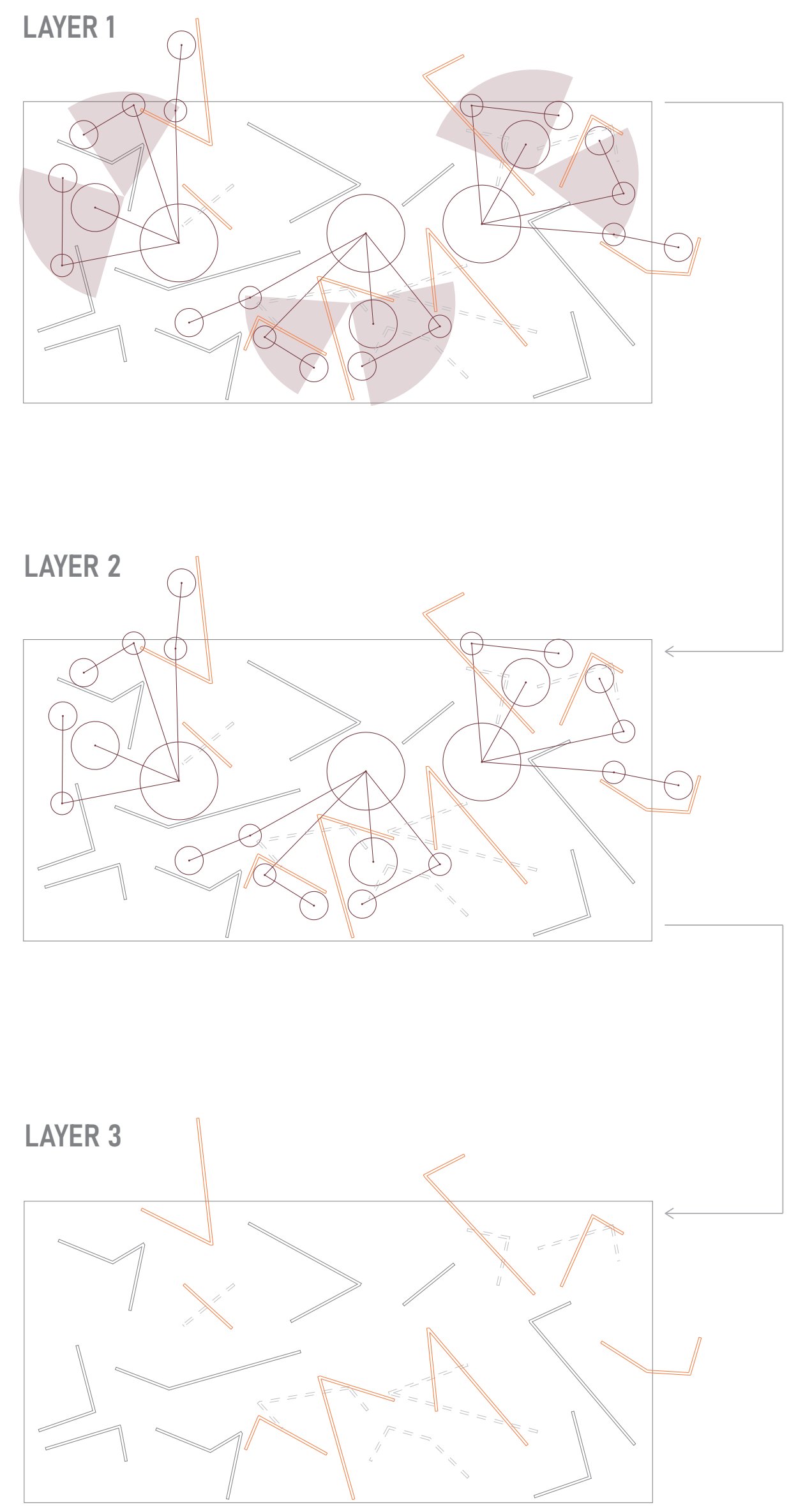


\section{CIRCULATION}

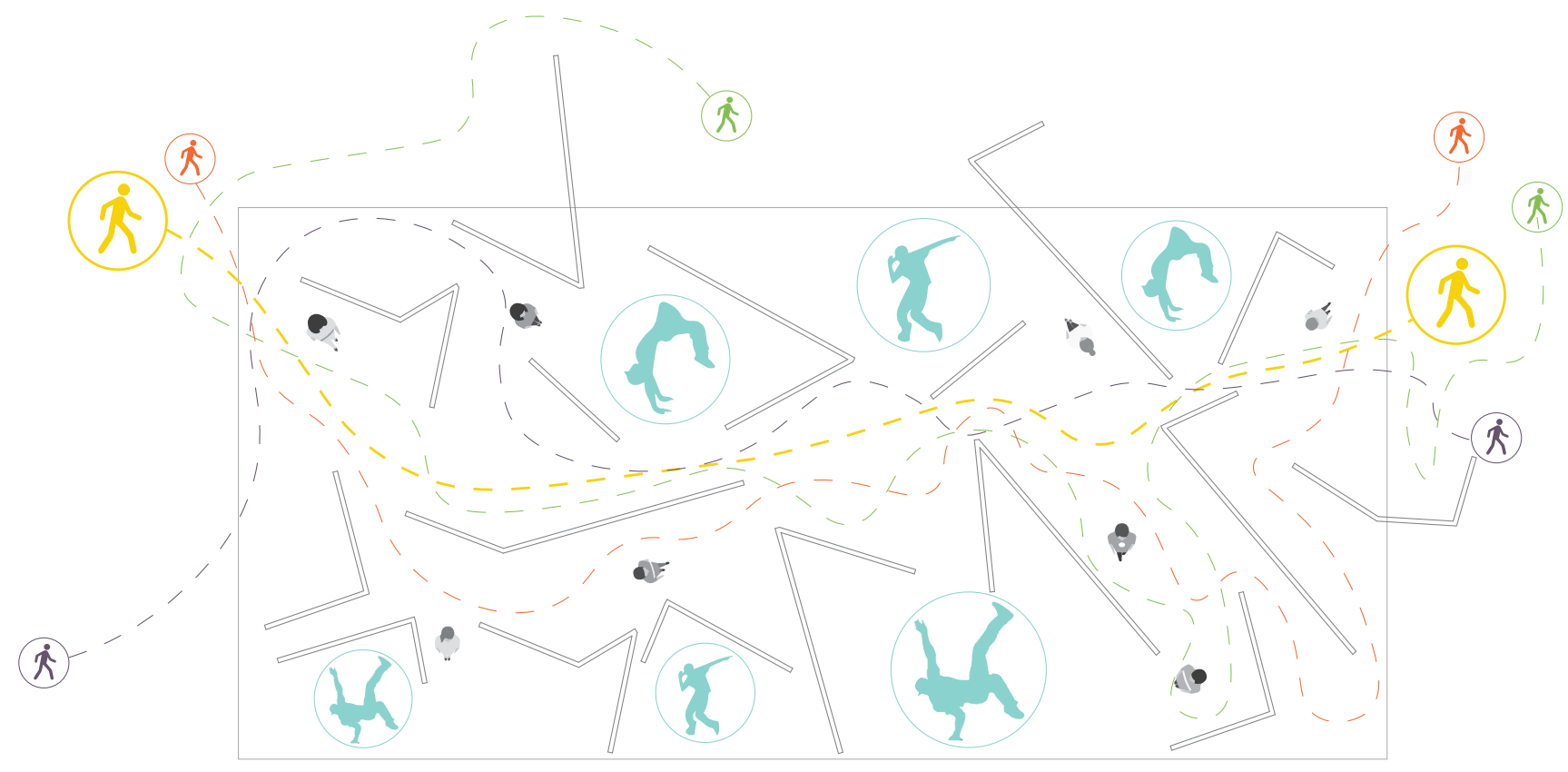

When designing this pavilion, circulation becomes key to consider. The ease with which the dancers move from space to space becomes important. There also needs to be allowances for potential public access while the dancing is occurring.

\section{TRANSPARENCY}

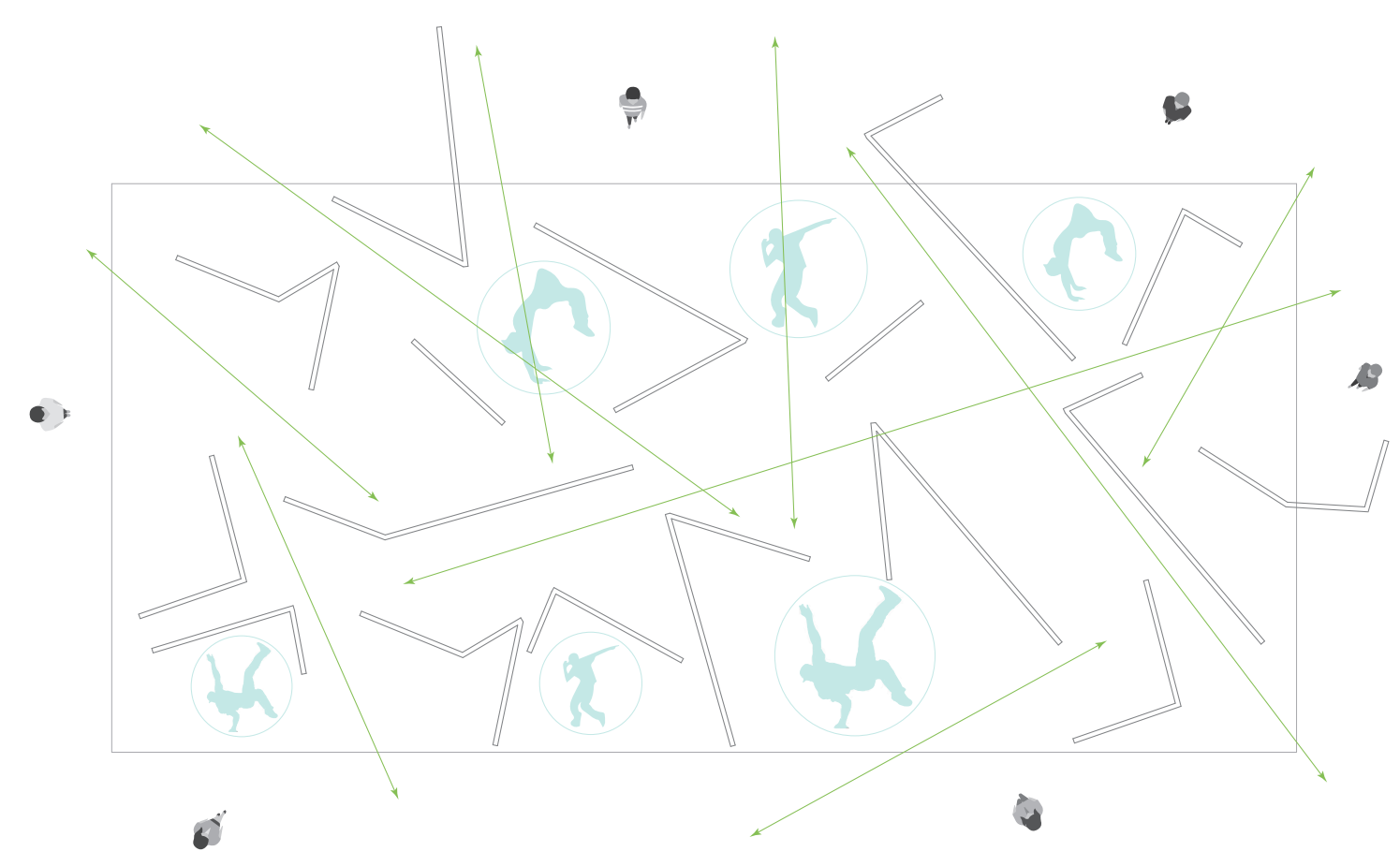

The idea behind this pavilion is to expose the public to the art of La Comuna 13. This is why the exterior shell is mostly glass and transparency becomes significant.

Focusing on how far into the pavilion the public can see and which areas are exposed. The dancing is located mostly around the periphery which makes it easier to see. 


\section{THE SHELL DEVELOPMENT}

\section{STAGE 1}

Focused on wall placement wihtin pavillion
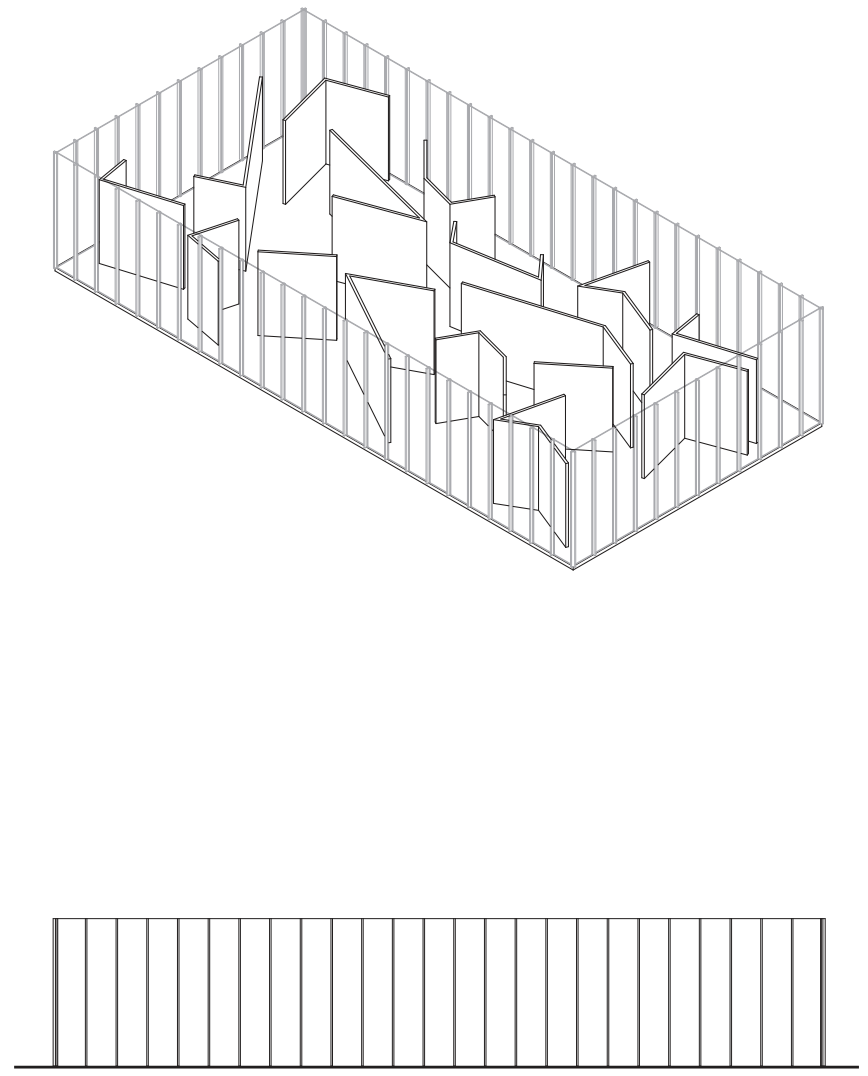

ELEVATION

\section{STAGE 2}

Exploring walls penetrating glass walls
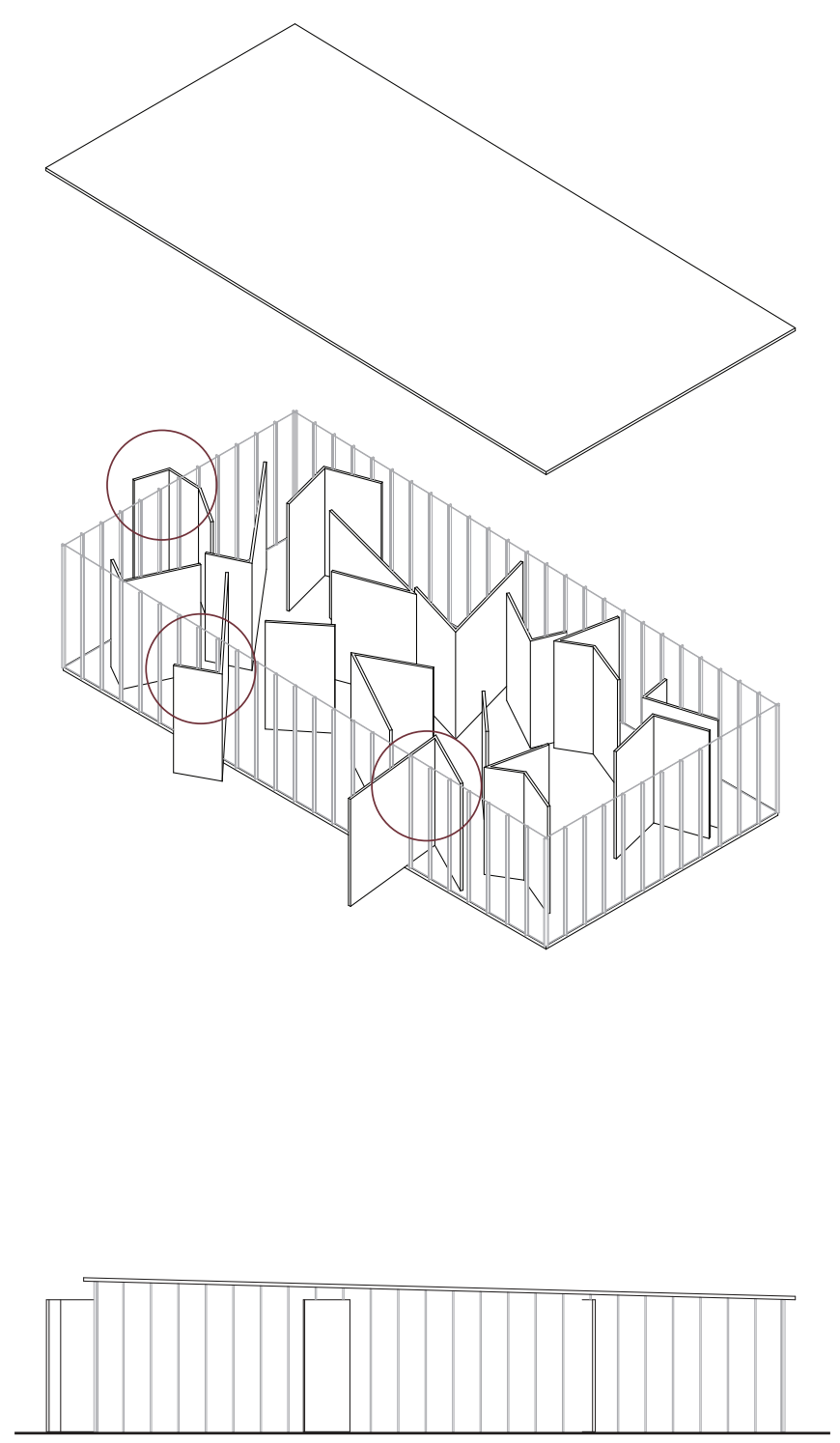

ELEVATION
STAGE 3

Increased penetartion through glass walls + roof
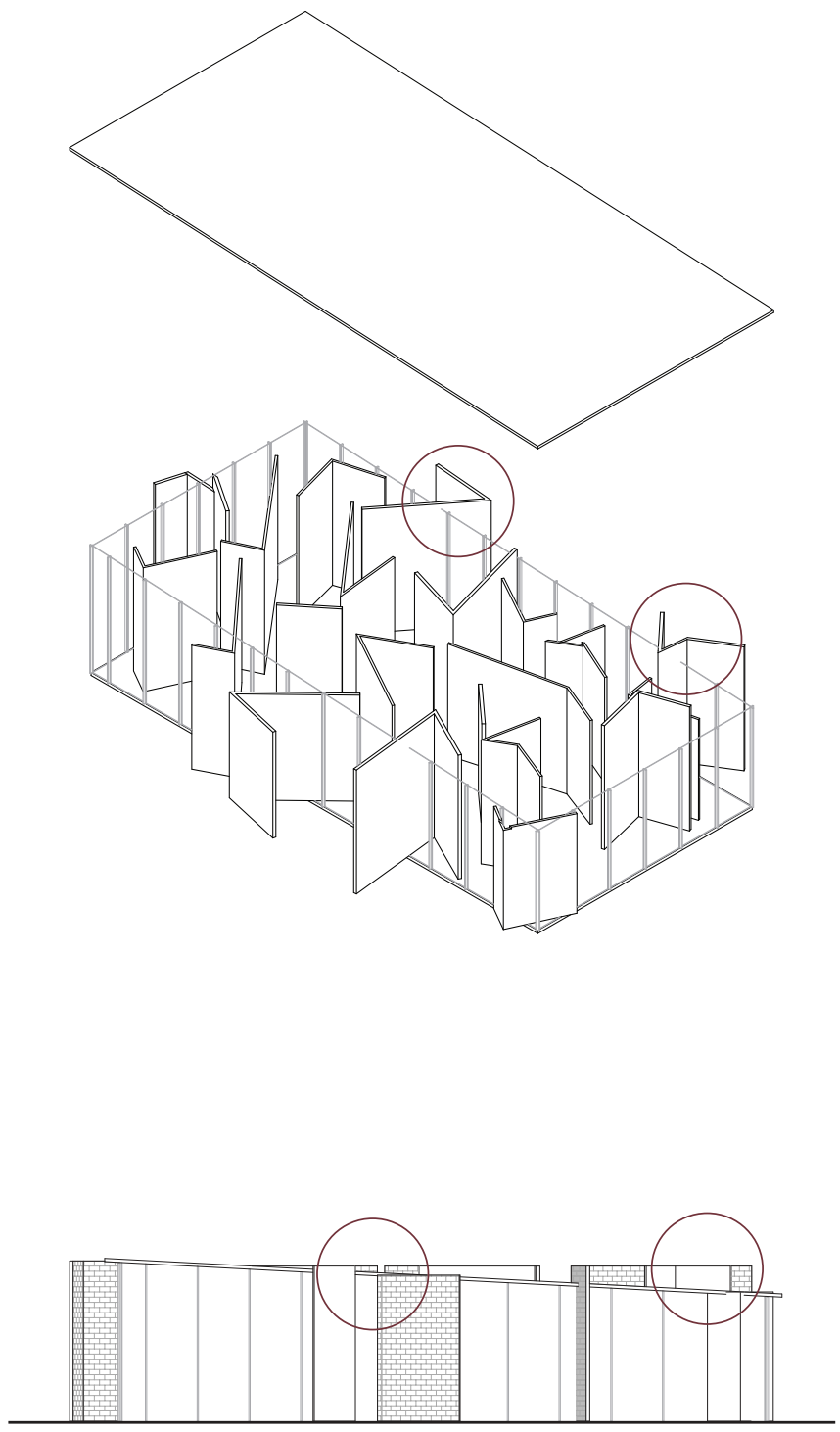

ELEVATION 


\section{FINALIZED DANCE STUDIO SHELL}
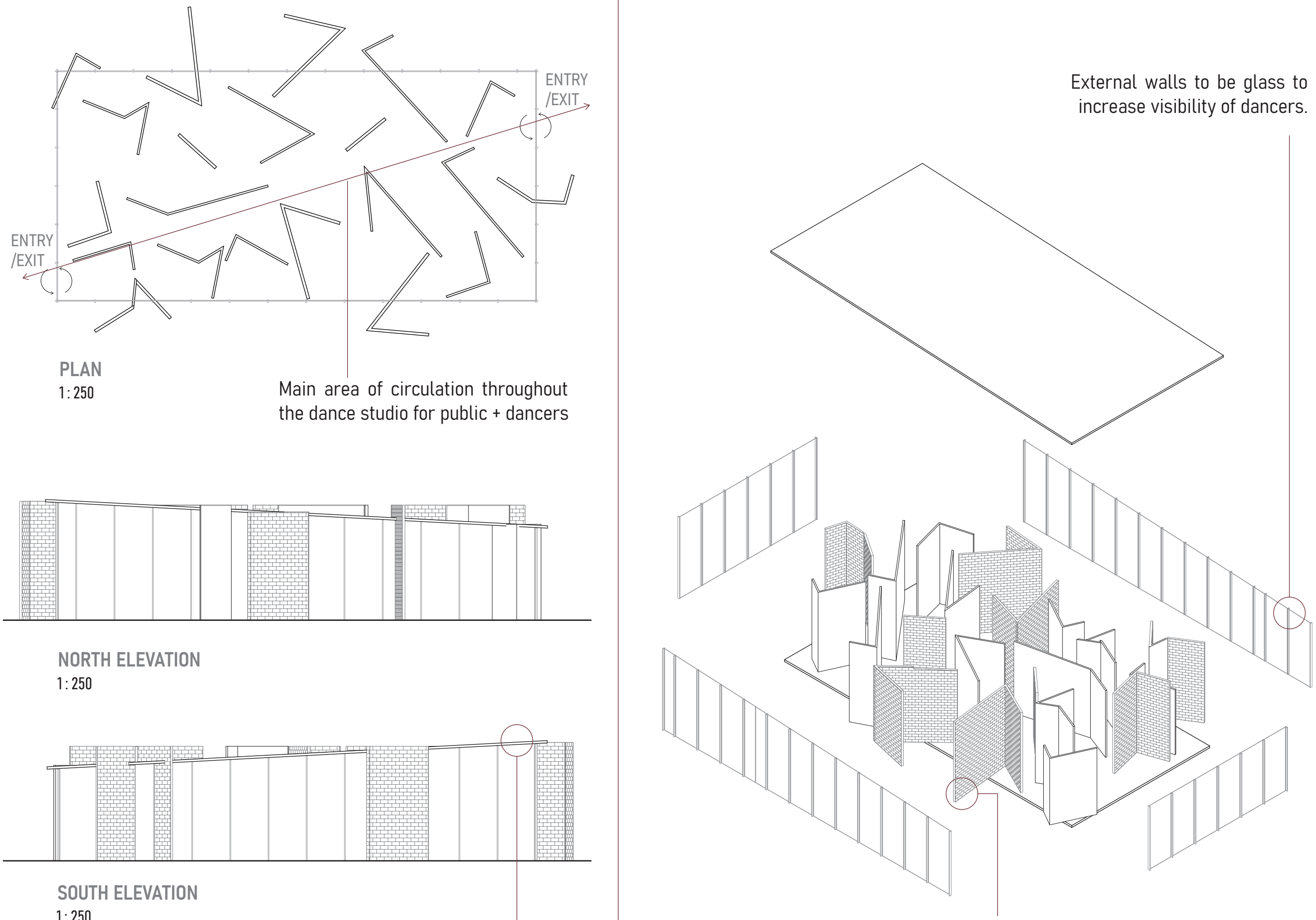

NORTH ELEVATION

$1: 250$

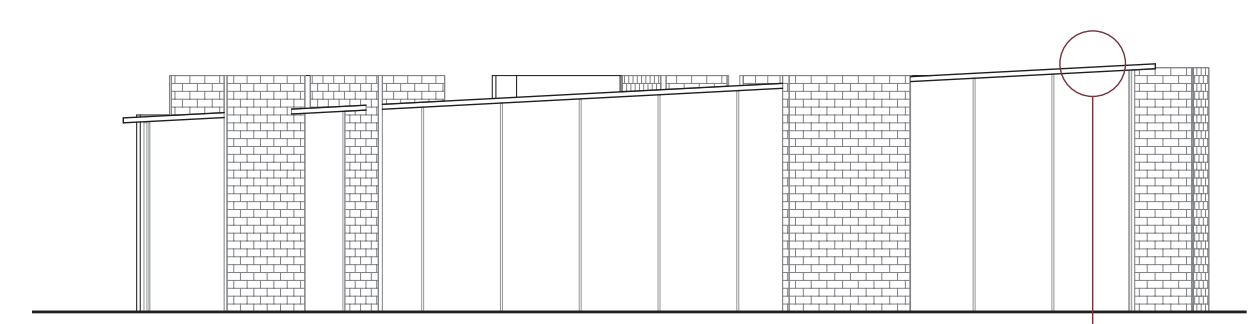

SOUTH ELEVATION

$1: 250$

Sloped roof to account for large amounts on rainfall on site

Continuing the use of brick walls to provide the canvas for the street art. All walls which penetrate the envelope were brick for this purpose 


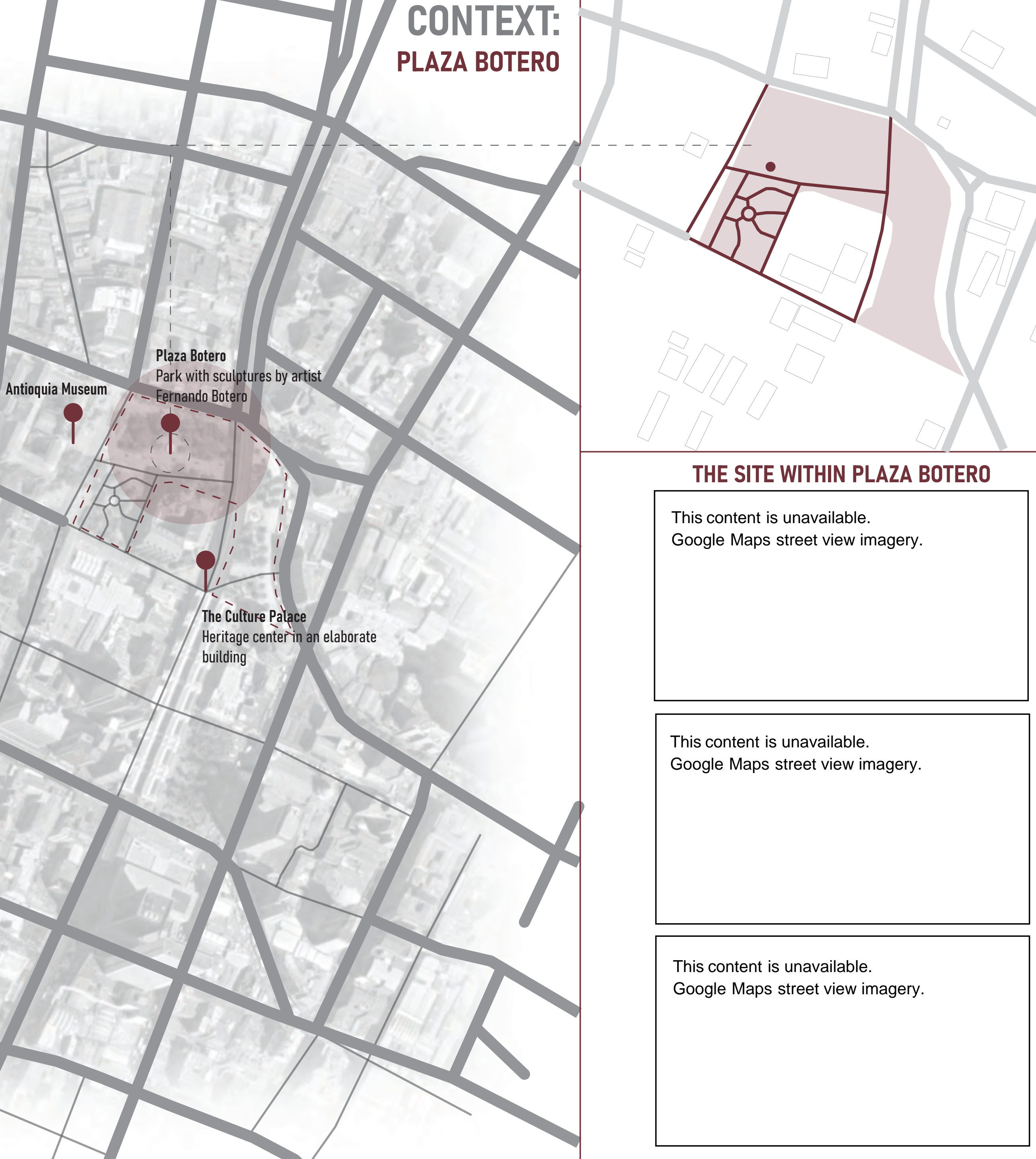




\section{PAVILLION ON SITE}

Plaza Botero is the chosen site of the Invited Encounter' for its expansive area (as previously discussed) but also because it is already a place known for art due to all the famous sculptures located throughout. This design is simply introducing a different art form to the site.

It is also an area with heavy foot traffic and visited a lot not only by locals but also tourists so there would be great exposure for the community here. It is a large flat site which also made it easier to position an architectural intervention such as a pavilion without needing to disturb the site.
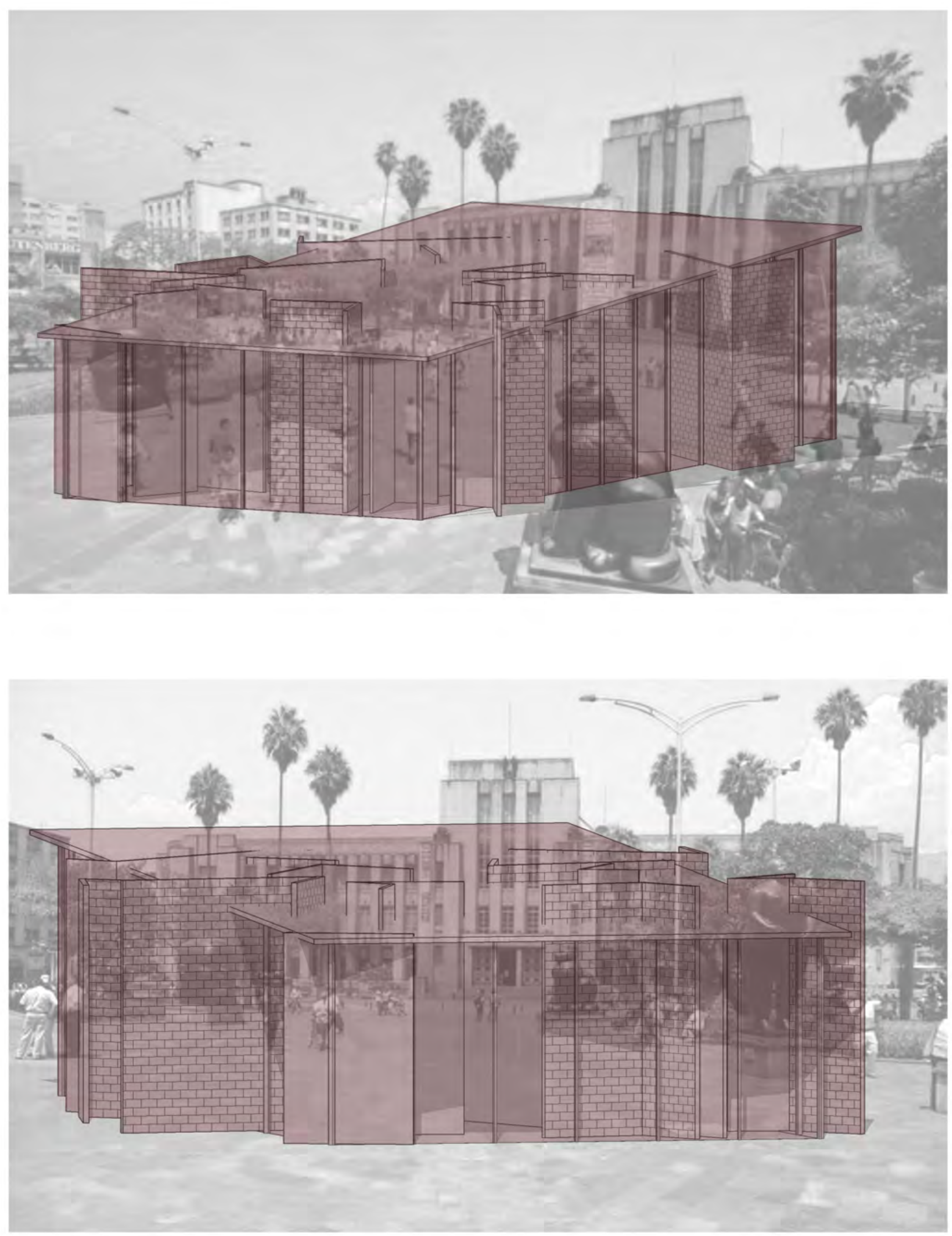


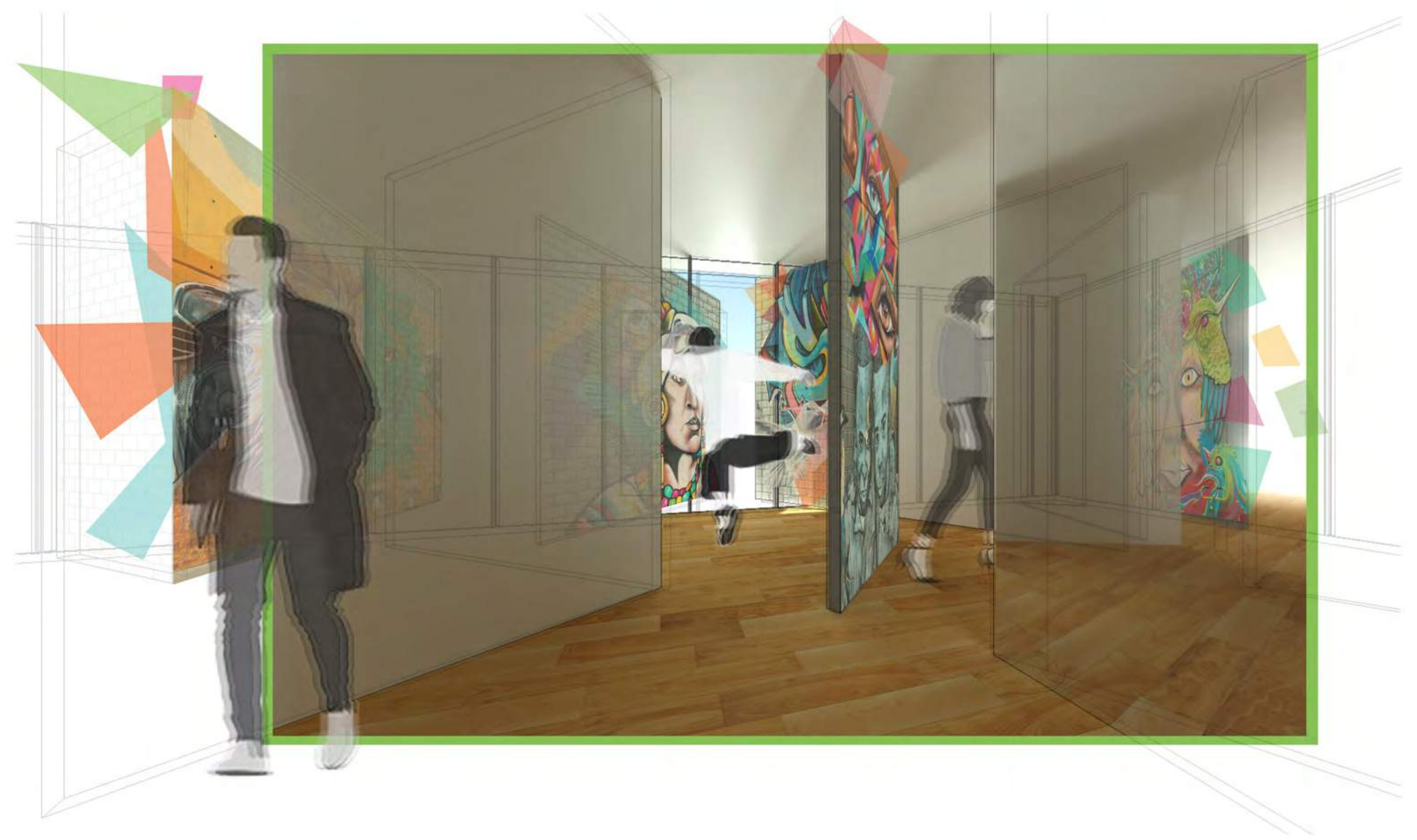




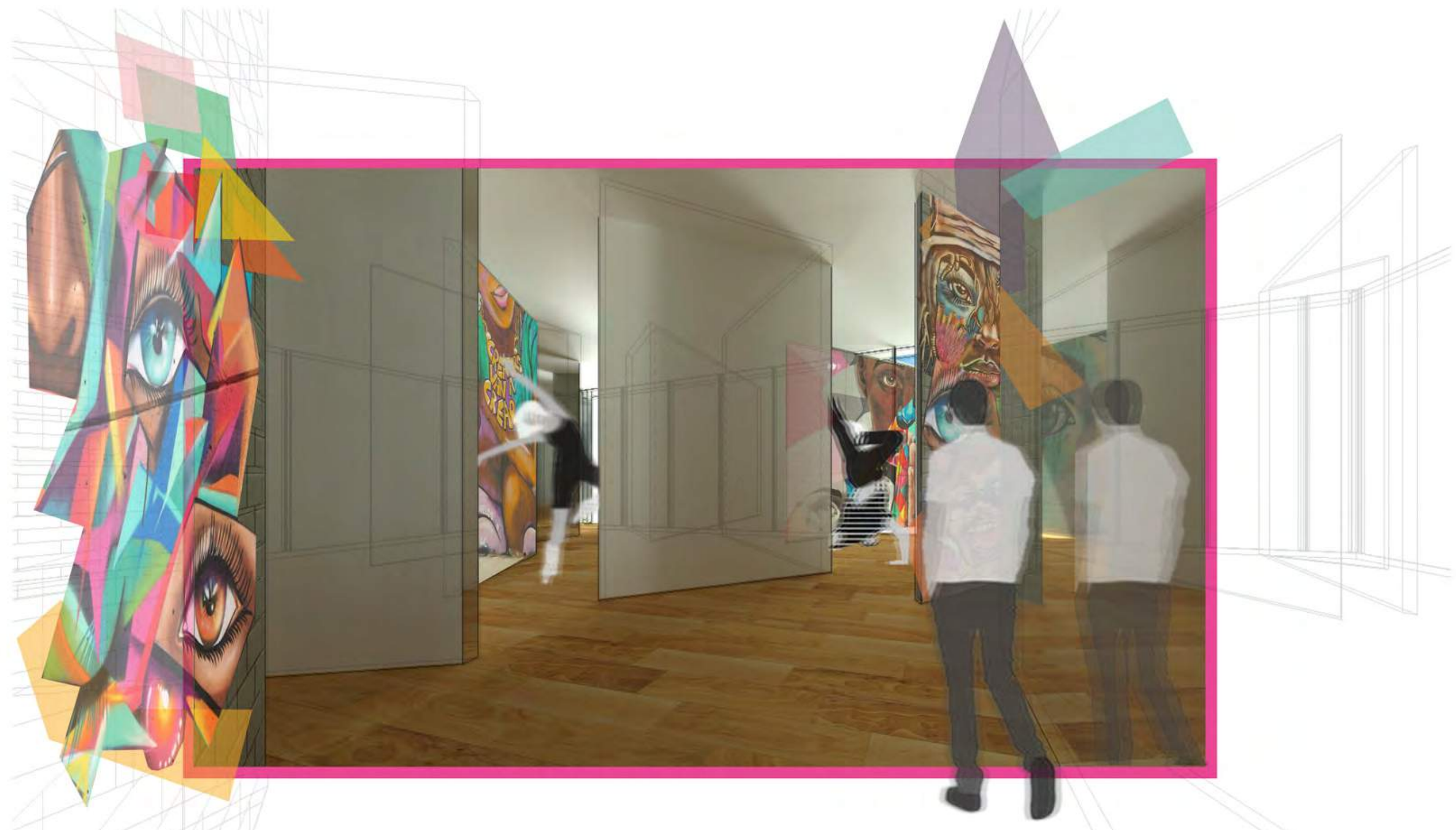




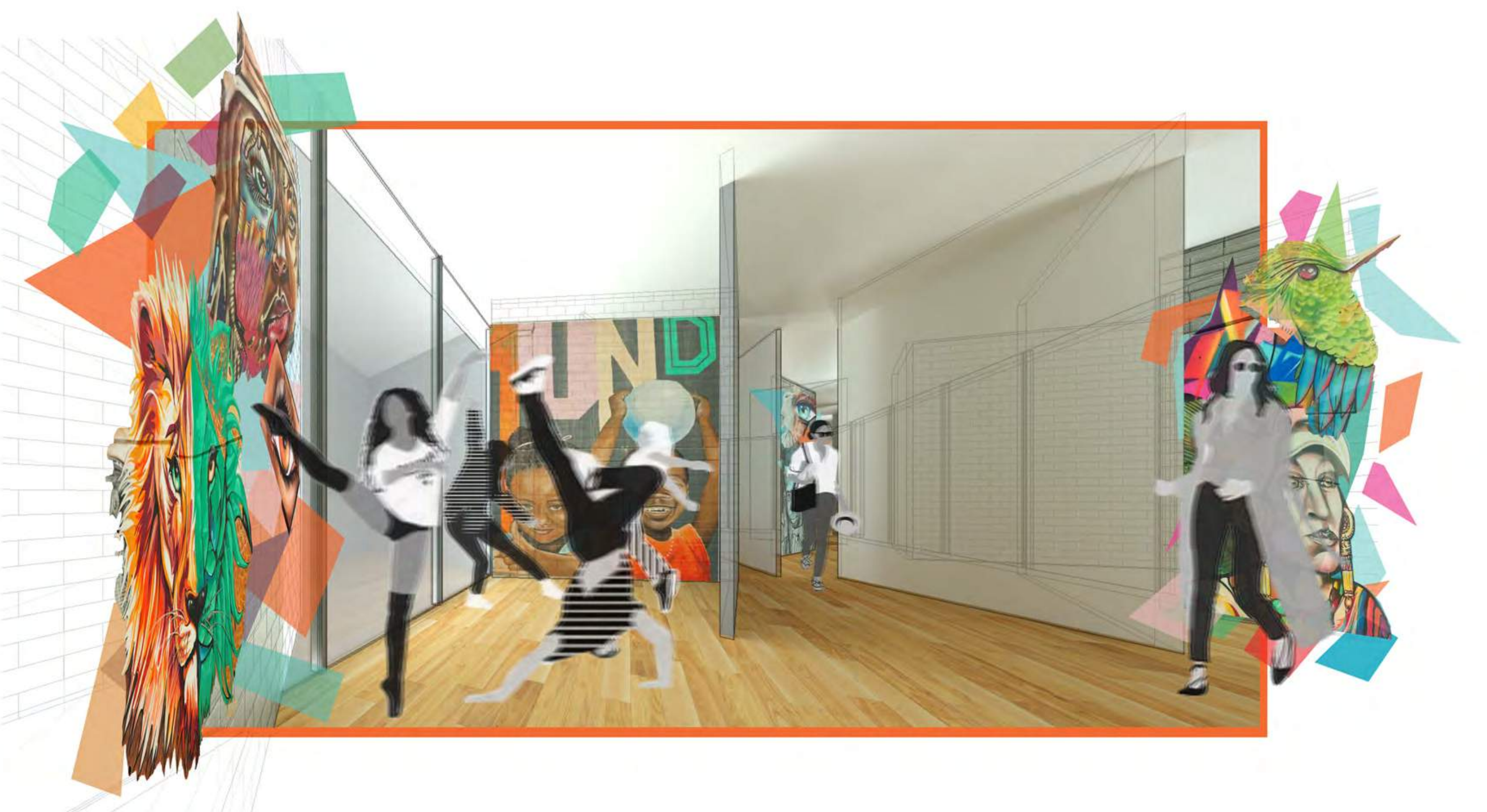




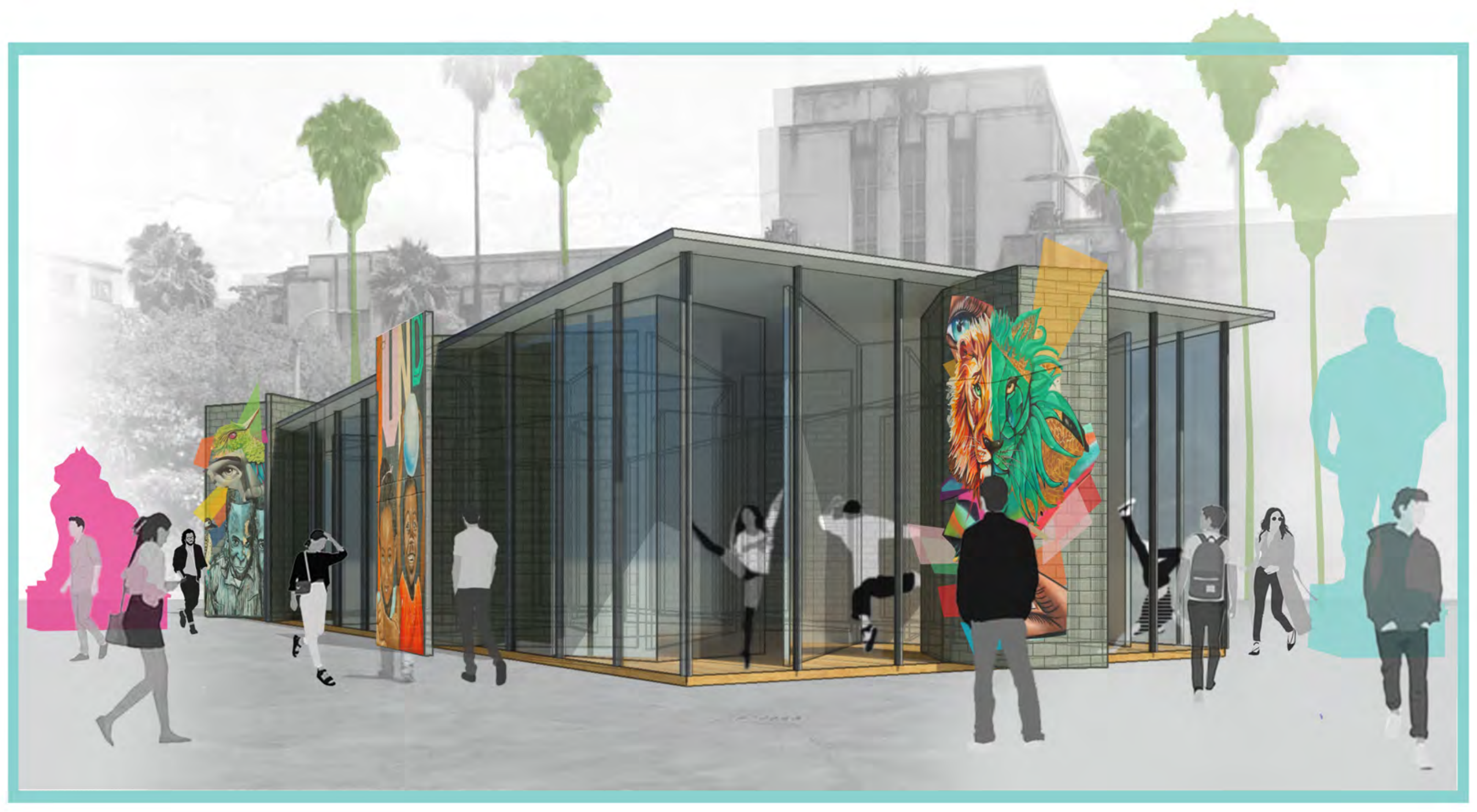




\section{Conclusions + Reflections}

\section{Conclusiones + reflexiones}

The motivation for this concept aligns with that of the Forced Encounter in terms of wanting to bring the community from the margin to the city centre. This time, however, creating an invited encounter where the wider public is extended an invitation to enter into the space of La Comuna 13 and witness their expression through the art of dance as well as painting. In this exploration, the floor/ground was as important as the wall. An element of exposure was also be considered, as the idea would be that the public should be able to watch the dancers from La Comuna 13 perform.

When it comes to developing this concept further, the circulation and inhabitation of the space could be further progressed in order to make the space more functional. There is also room for further design in terms of the shell. Things such as openings, details and the structure itself could be further refined but the key focus in this research was the interior architecture.

The key aims of this design were exposure, visibility and enabling an interaction between La Comuna 13 and the wider public. In its conceptual stage, this design has focused well on these aims. This pavilion becomes a space for the community to not only be seen performing but it also offers them an opportunity to engage with the wider public and tell their stories through more than just the medium of art. 
"El impacto que ha tenido La Comuna 13 gracias al arte ha sido increíble. Yo creo que esta podría ser de las comunas que mas aceptación tiene hacia las diversas formas que el arte presenta." - Kbala

\footnotetext{
"The impact La Comuna 13 has had thanks to art has been incredible. I believe that this could be one of the communities that has the most acceptance towards the various forms that art has to offer."
} 


\section{A HIDDEN ENCOUNTER}

CONCEPTO 3: ENCUENTRO OCULTO 



\section{Concept Three Introduction}

\section{Introducción al concepto tres}

This final programme exploration originated from the realisation that the people in La Comuna 13 create a sense of identity within their neighbourhood through art while living in poverty. This realisation led to the programme of providing the community with a new place to live which better accommodates them but also retain the opportunity for the expression of who they are.

Themes such as place identity and a sense of belonging were discussed in previous chapters will be drivers for this third concept.

With sustainability at the forefront of this design, it was decided that an existing abandoned building in Medellin would be used and turned into high density housing through adaptive reuse. This means only the interior architecture becomes the focus. This programme is purely a space for the community, hence the hidden nature of it. It is noteworthy that many buildings are abandoned in Medellin due to a lack of resources from the government and provide many opportunities for further developments.

The process of creating the design for this concept was heavily affected by some of the theory behind high density housing design such as minimum areas legally required for each space within the habitation as well as the number of people the apartments catered to. This then began to merge with some of the ideas explored in the other concepts such as layering in terms of layering the shapes on top of plans and using these to alter the apartment layout.

Further reading into the design of high density living, adaptive re-use and family life/ structures in Colombia was followed up by a precedent study and then the site selection. This involved finding an abandoned building and creating a digital model of the shell to work within. The design process then starts by first establishing the areas which are going to be used and these are then tested through an iterative process. This evolves into the layering of shapes on top of these plans and allowing them to disturb the wall layout in order to arrive at floor plan or wall layout to take into the developed design stage. 


\section{HIGH-DENSITY HOUSING}

Vivienda de alta densidad

Due to the community having a large population in a relatively high-density housing complex, the new housing design will follow suit in order to accommodate a significant portion of the community while help maintain the current style of living where community spaces are considered important and where families interaction with privacy are maintained. To do this, it is important to understand the theory and planning which are involved in the design of the interiors of high-density housing in Colombia.

As established in Chapter 1, this community may not be satisfied with their current living environment. Tao Ho states that it $\mathrm{i}\urcorner \mathrm{s}$ possible for people to live under crowded conditions, and still lead dignified, happy and productive lives. The problem then becomes to design with a thought beyond the provision of a unit of space per person (Ho, 1975, p. 377). This challenges the idea that to design housing, one must think of the space required for each activity and design according to that. Ho argues that the numerical size of the living unit is not the key design criterion for high-density housing. What is most important is the design of the basic unit in relation to its overall social environment which makes it possible for people to live a happy and productive life (Ho, 1975, p. 378). Thinking of unit sizes will still be critical for this design-led research however, seeing as the living units will be designed within an existing building, a consideration into communal and open spaces will be crucial to ensure the provision of a social environment. When it comes to the sizing of the units, the idea is to create a series of different typologies which all cater to different needs in terms of space and occupancy. James Tice suggests that housing is arguably the most appropriate arena for typological studies. The continuity of basic dwelling themes such as the need for pri- vacy and community, access to light and air, and proximity to nature has given rise to diverse housing types that constitute a general housing framework that remain relevant today (Tice, 1993, p. 163). There are many existing housing typologies; some are project specific and others are well-known due to their frequent use in housing projects. Due to the uniqueness of this research, the housing typologies will be part of the design process and will be created to cater to the community of La Comuna 13.

The issue then arises of how to approach the design of the units without beginning with numerical values. Tao Ho suggests looking at the unit as a "basic human bubble". This bubble is more than a physical shelter. It is a basic human environment in which a man can live with dignity, productively and happily. It is not a finite unit which can be defined in terms of numbers such as so many square meters per person. There is a fundamental assumption of this "basic human bubble" and it is that it consists of constant factors and change factors. The constant factors are those common to all human beings that are not affected by time and space. For example, shelter, food, air and sleep (Ho, 1975, p. 379). These constant factors would all be things which would normally be considered when undertaking housing design or any interior design for that matter. It is the change factors that have the potential to create difficulties in the design process. Ho explains change factors by giving the example that man needs food to live, which is a constant factor. But what food he needs and how and when he eats are dictated by change factors. These change factors are things such as the man's nationality, historical background and economic necessity (Ho, 1975, p. 380). These will be the things which will be most important to consider in the design process as these are things which
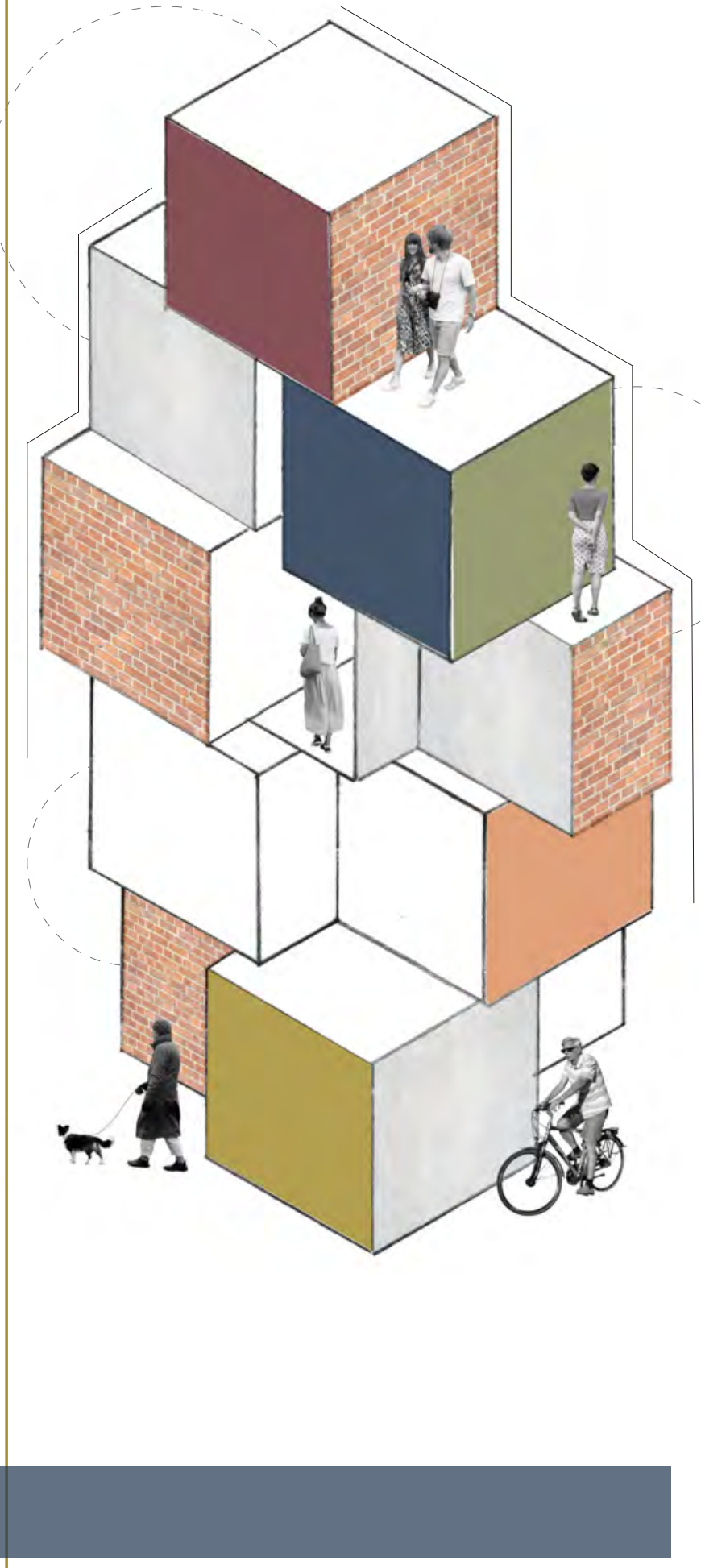
would vary not only from person to person but also between family structures.

Christian Schittich discusses this idea that although social structures have changed considerably in the past decades, the typical apartment floor plan is still almost exclusively designed for the needs of the "nuclear family". It is almost as if today's variety of lifestyles is not imposing an imperative for specialising floor plans (Schittich, 2012, p. 9). This highlights the importance of considering the potential social structures of La Comuna 13 and the needs of each housing typology. There are also the changes to the purpose and use of different zones within the housing units. Schittich explains how the kitchen is no longer a space simply just for cooking but it is also the place where friends are entertained. It is a multiuse, social space which sometimes assumes the functions of the living room. Given the changes to the function of the kitchen, the living room has also taken on a different status. When it comes to the bedrooms, today one bedroom per person is the norm. Raised expectations of withdrawing to a private space have transformed it from the original small sleeping space into a multi-functional living, sleeping, and working space (Schittich, 2012, p. 30). These are all standards and circumstances which may not be true for the community of La Comuna 13 as they live in poverty and their current housing is just the bare minimum. But for the new housing design, these ideas about space usage outlined by Christian Schittich are important to consider as they will influence the design and planning of the units.

The design of the housing typologies will be a key aspect in this research. Tao Ho outlines that in the design of the housing typologies with a community, one important factor must be kept in view: the creation of certain common elements at the very beginning such as a market place, shops, community centre, in which people may participate at the same time. This will create a feeling of community continuity and a sense of belonging (Ho, 1975, p. 381). Due to the artists in La Comuna 13 already having art and dance classes set up for the younger members of the community, it will be paramount to provide a community centre in order to enable things like these classes to continue. It will also offer a place where different age groups can come together and interact. Giving the older population of the community an opportunity to have an input in the themes and ideas behind the art which represents their memories and their histories whilst also informing and educating the younger population. Also creating a space where their artistic expression is encouraged and enabled.

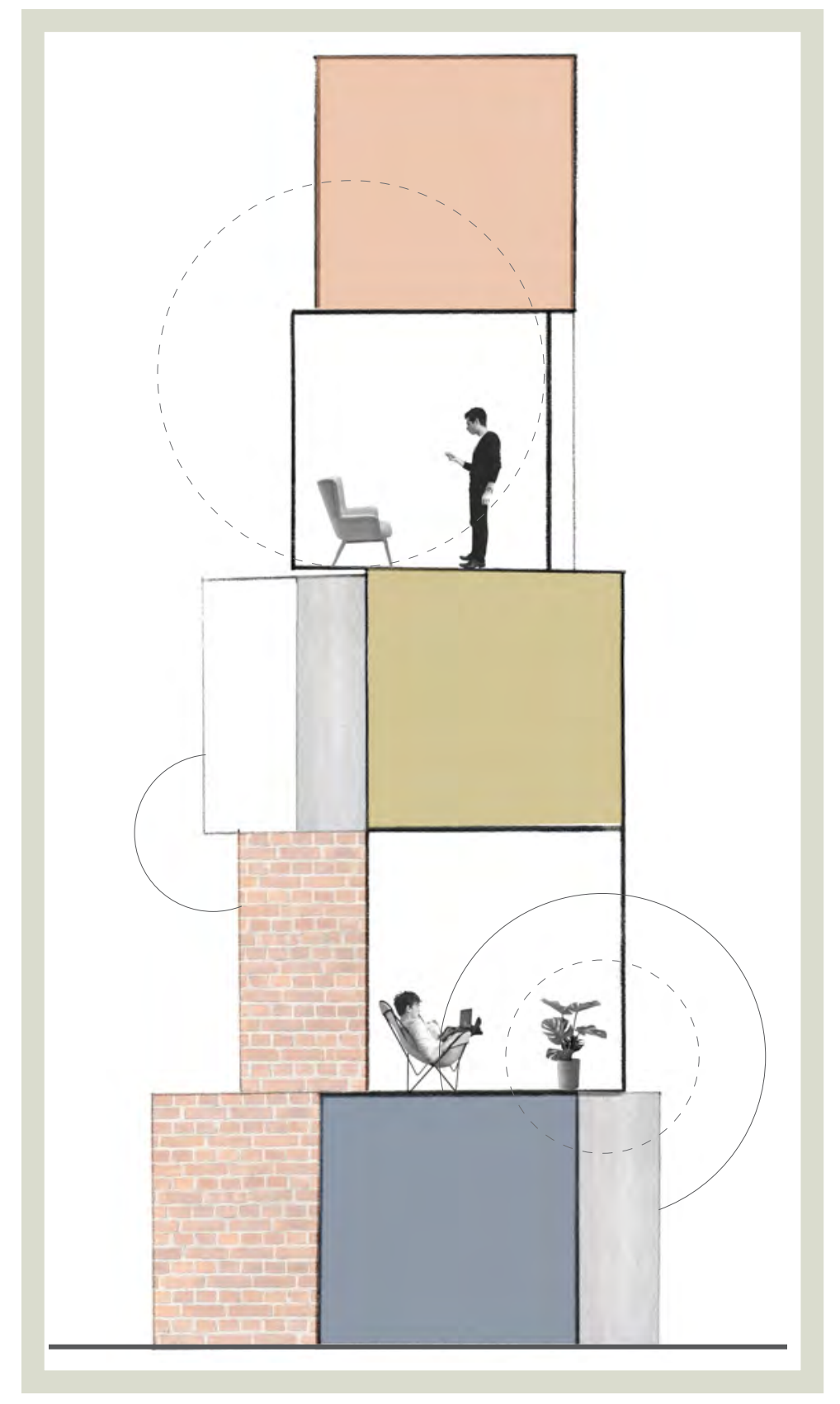




\section{ADAPTIVE RE-USE}

Reutilización adaptable

Adaptive reuse today may be summarised as a design-based intervention aimed at bringing new life to a building - often neglected, abandoned or misused - which, for any reason - be it historical/artistic, cultural, or economic - has been considered worthy not to be demolished (Lanz, 2018, p. 4).

In the context of this design-led research, the building being adapted is a large apartment building which has been abandoned. The idea of adaptive reuse is almost exclusive to interior architecture. Francesca Lanz suggests that working within pre-existing buildings is the distinguishing nature and the specific realm of interior architecture. The contribution of interior architecture to the theory and practice of adaptive reuse is not so much related with the act of designing the "inside" of something or inside something but rather lies in its approach to the design process as an enfolding and fabrication of space which makes inhabitation possible (Lanz, 2018, p. 5). This introduces the idea of "re-inhabiting" and bringing a completely new habitation aspect to the building.

There is a lot more to be considered in terms of adaptive reuse than creating a new interior architecture or re-inhabiting a building. Lanz illustrates how re-inhabiting a pre-existing space opens up the discourse on reuse to issues regarding the relationship between people, places, memory, identity, and belonging (Lanz, 2018, p. 6). This not only highlights the importance of the ideas discussed in Chapter 2 but it also presents the implication that these are things which must be considered. The design process must now not only involve the memories which are embedded in the art of the community, but also the memories attached to the building being adapted. This building will already have an identity of its own and it becomes a negotiation between this established identity and the new identity which will be introduced through the adaptive reuse, the design of the interior architecture and the community.

Graeme Brooker explains how these relationships generate a dependency based on the intimacy and correlation between the new and the old (Brooker, 2016, p. 7). The idea of identities is one which is significant for both the community and the adaptive reuse of the building. Brooker argues that the creation of significant narratives, accounts, stories of space, its occupants, and their inhabitations can be resolved through the construction of particular identities (Brooker, 2016, p. 9). It will be pivotal in the design process to consider the idea of identity in terms of materiality and space to create an interior architecture which this community not only identifies with but also can feel a sense of belonging towards. The concept of memory also becomes significant with the introduction of the new memories through the street art but also the memories which the building already holds. Liliane Wong portrays how the removal of even a simple partition leaves a tell-tale trace on both the ground and the ceiling: a scar defined by the depth and thickness of framing members and finishes (Wong, 2016, p. 136). This idea of scars left behind can not only be closely tied to memory but also to La Comuna 13 . Their violent past has left a significant scar in their history and their memories. When it comes to adapting a building, a similar event would take place where through the act of removing aspect of the existing interior, traces and scars would be left behind Peter Bullen and Peter Love argue that the most successful adaptive reuse projects are those that respect and retain a buildings significance and add a contemporary layer that provides value for the future (Bullen \&
Love, 2011, p. 33).

Liliane Wong approaches adaptive reuse as a relationship between a guest and a host. She describes how the shell-type host structure permits both limited and unlimited actions within the confines of its infrastructure. Interior conversions typically respect the existing structural system, the floor plates, and the envelope. Within these constraints, the host of this type acts as a blank slate for unlimited new design strategies. The shell-type host acts simply as a box for the introduction of new spatial experience (Wong, 2016, p. 162). This explains the nature of this design-led research as it aims to work within the shell of an abandoned building to introduce an entirely new programme and use. Adaptive reuse presents key themes such as memory and identity which are deeply embedded in the practice and will be influential in the design process. These are themes which are already also deeply embedded in the community at La Comuna 13 and so this design will become a negotiation between the memories and identities of both the community and the building. 


\title{
HOUSING + FAMLIY LIFE IN COLOMBIA
}

\author{
Vivienda + vida familiar en Colombia
}

\section{Housing}

Having the background information on the design of high-density housing and adaptive re-use, it then becomes key to develop an understanding of the design of housing in Colombia as well as the family structure.

When looking at housing in Colombia, Perez-Perez discusses the design of low-cost housing designated for lower socio-economic communities which tends to be built at a higher density. He explains how a house which is adequately designed based on the characteristics, needs and expectations of the users, their environment and the relationship with the city, is essential for psychological and social development, favours urban sustainability and contributes to raising wellness at a lower cost (Perez-Perez, 2015, p. 67). This begins to outline the implications of a housing development beyond just the users and the building but also the wider community and site. He raises the positive impacts on urban sustainability but also in this design-lead research using adaptive re-use hopes to further address the issues of sustainability. Perez-Perez outlines the results from a survey carried out in order to analyse the quality of life in this low-cost housing which identified three key aspects which either improve or hinder it. These being: access to basic public services; overcrowding, understood by the number of people sharing a room (more than 3); the possibility of accessing a formal education and, therefore, the possibilities and economic independence of users in the home (Perez-Perez, 2015, p. 68). These become significant aspects to consider when selecting a site and also when beginning to design the spaces within the housing. The way in which Perez-Perez approaches his research is by framing the design of housing around both the needs and expectations of the users. He explains how expectations are then the articulating element that motivates, favours and guarantees users (understood as individuals, their families and, therefore the community to which they belong) their access to a better quality of life. Therefore the satisfaction of expectation is necessarily linked to the progressivity of housing. These expectations, however, constitute a projection towards the future, reflecting the wishes of the users and, therefore, the continuous evolution of the home. (Perez-Perez, 2015, p. 70).

Having understood and analysed these ideas of expectations, Perez-Perez then outlines some strategies which could be implemented in order to improve design according to the way in which these communities live their life. A strategy which is not necessarily specific to design is the promotion of adequate user participation at different scales. This is due to the findings that housing developments which are not integrated into the context, without an adequate design of outdoors spaces, lead to users who want more green areas, public spaces and facilities for social interactions, taking over public areas in order to make them communal, leaving their mark of appropriation (Perez-Perez, 2015, p. 73). This is interesting because this is essentially what has happened in La Comuna 13. There is no designated space for the community to create their art and express themselves so they have taken over the communal areas in the streets to do so. This only highlights the importance of providing this community with a communal space such as a work- shop where they can go to create their art without affecting the wider community. A strategy which is more specific to the design of the spaces within the housing is inclusion of a space within the home where the users can carry out "productive activities". Perez-Perez explains how providing for the incorporation of productive activity in housing is a requirement which is demonstrated by the number of cases in the survey where the living room or dining area have been sacrificed in order to carry out these productive activities (Perez-Perez, 2015, p. 74). This becomes very important because as stated in Chapter 1, many of the people in this community craft products within their homes which they can then sell to tourists on the streets. Providing them with a designated space where they can do this is imperative to facilitate their source of income and through this, improve their quality of life.

There are various other design strategies and criteria detailed by Pere-Perez which are highly relevant to this design-lead research and they are as follows:

- Allow the inhabitants to remain in central urban areas, facilitating the enjoyment of existing services and infrastructure, as well as maintaining close proximity to work and study.

- Include public spaces and green areas, delimiting the communal and private public spaces, and where high densities are achieved combine the advantages of single-family and multi-family living. 
- The design should benefit the quality of neighbourhood relationships, the social climate, the generation of exchange networks and mutual aid, favouring feeling of belonging.

- Incorporate in the house areas that families can adapt to carry out activities that allow them to increase their income.

All of these strategies create links not only to the previous research into high-density housing in this chapter but also to some of the concepts explored in previous chapters such as the ideas of identity and sense of belonging created within this community.

\section{Family Life}

Having established some background knowledge about the design of housing for low socio-economic communities in Colombia, it becomes paramount to develop an understanding of family structures and dynamics in the Colombian culture.

In their research, Neiza et al. outline the results from a study which showed that the most outstanding characteristic of the Colombian family is the coexistence of the various structures, among which some are traditional and some are modern. There was an investigation which compared the prevailing family structure in 720 families from four cities in Colombia: Bogota, Medellin, Baranquilla and Cali. The result show that the nuclear structure prevails (54\%), followed by the extended (17\%) and the female as single parent $(14 \%)$ in all cities (Neiza et al., 2017, p. 72). This begins to set up some significant factors to consider when designing the housing as there is clearly a requirement for different types of housing to accommodate the different family structures. This will translate into the design in the form of space allowances and layout of each housing typology. Some comparative studies were done and Neiza et al. concluded that changes have occurred and continue to occur in the family structure in Latin America and Colombia, and these changes imply a progressive decrease in the nuclear structure, to make way for the new family organisations, such as the extended and single parents, especially female (Neiza et al., 2017, p. 72).

It is also important to consider not only changes in family structure but also the variations which can occur within each individual family structure. Neiza et al. discuss how although two families may have the same structure, in reality, they are different. For example, in the case of families with a nuclear structure, in one there are two children and in the other three, which generates changes in family dynamics (Neiza et al., 2017, p. 103). This reinforces the importance of space affordances as family dynamics may also influence the way in which the families or occupants use the spaces. Seeing as La Comuna 13 is located in the outskirts of the city and could be considered rural, it became relevant to understand if there are any differences when the family is rural. "It is common to find that in rural areas families share land or housing with other member or close people, so there is a third-party involvement in family dynamics, especially in raising children" (Neiza et al., 2017, p. 103). This can then influence design as this extended structure refers to the coexistence of other family members, in this case the proximity of the dwellings and the involvement in the coexistence meant they were considered extended family (Neiza et al., 2017, p. 103) which means that even if the people who are living in the dwelling are not related, they still view each other as family. This adds yet another dimension to the design process as there has to be consideration into the fact that the families in La Comuna 13 are likely to be large and follow this "extended" structure. The economic situation is another influential factor in family structure as it can cause multiple nuclear families to group together or the need for relatives to support other members of the family who need it which has been indicated by studies of the Latin American population (Neiza et al., 2017, p. 103). La Comuna 13 is a low socio-economic community which means that it is again likely they will follow this structure of multiple families living together or extended family living together in order to support each other economically. 


\section{MoMa Tower by Axis Mundi}

This is a project which takes inspiration from the randomly piled Brazilian favelas and Italian hill towns, the design offers a more accommodating flexibility for individual expression. The unit modules are assembled like pieces in a three-dimensional vertical puzzle around two cores that run the full height of double towers. Each unit module has a different exterior treatment based on a handful of iconic architectural styles, giving the irregularly stacked façade the appearance of a crazy-quilt vertical neighbourhood.

This illustrates some of the ideas discussed in the previous chapter in terms of high-density housing as the design has both the constant factors of the basic human needs but the change factors in the different sized units and individuality of the facade. It also exhibits ideas of layering not only through its irregular appearance but also through the act of stacking/piling the units on top of each other. The design as a whole resembles a piece of graffiti art with the changing shapes, colours and textures which makes it relevant to the work being carried out.

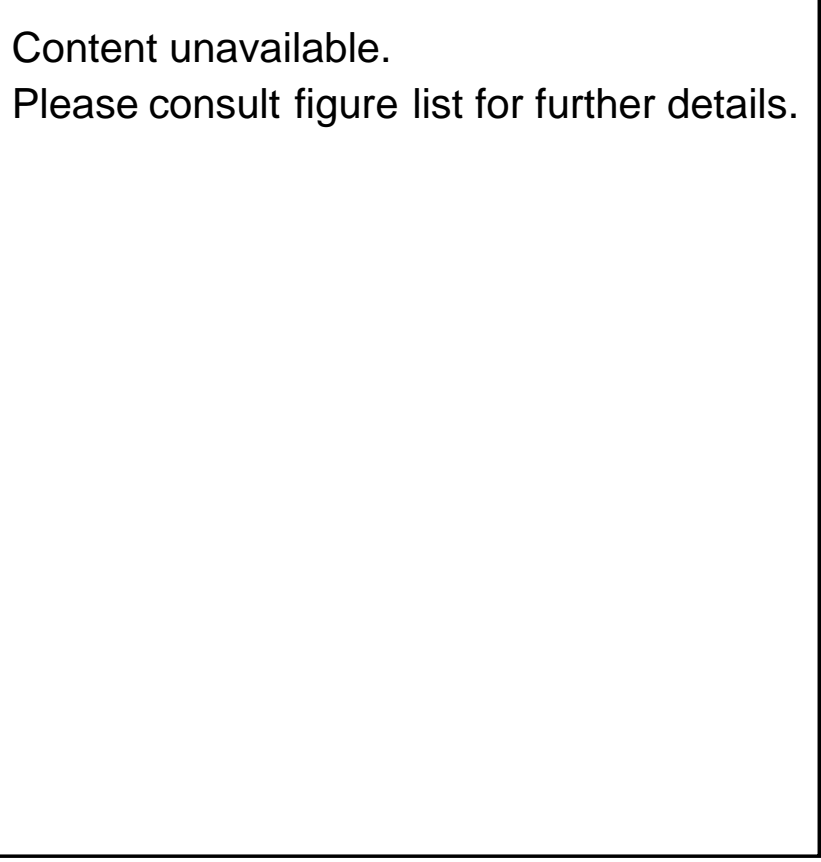

\section{Content unavailable.}

Please consult figure list for further details.
Content unavailable.

Please consult figure list for further details. 


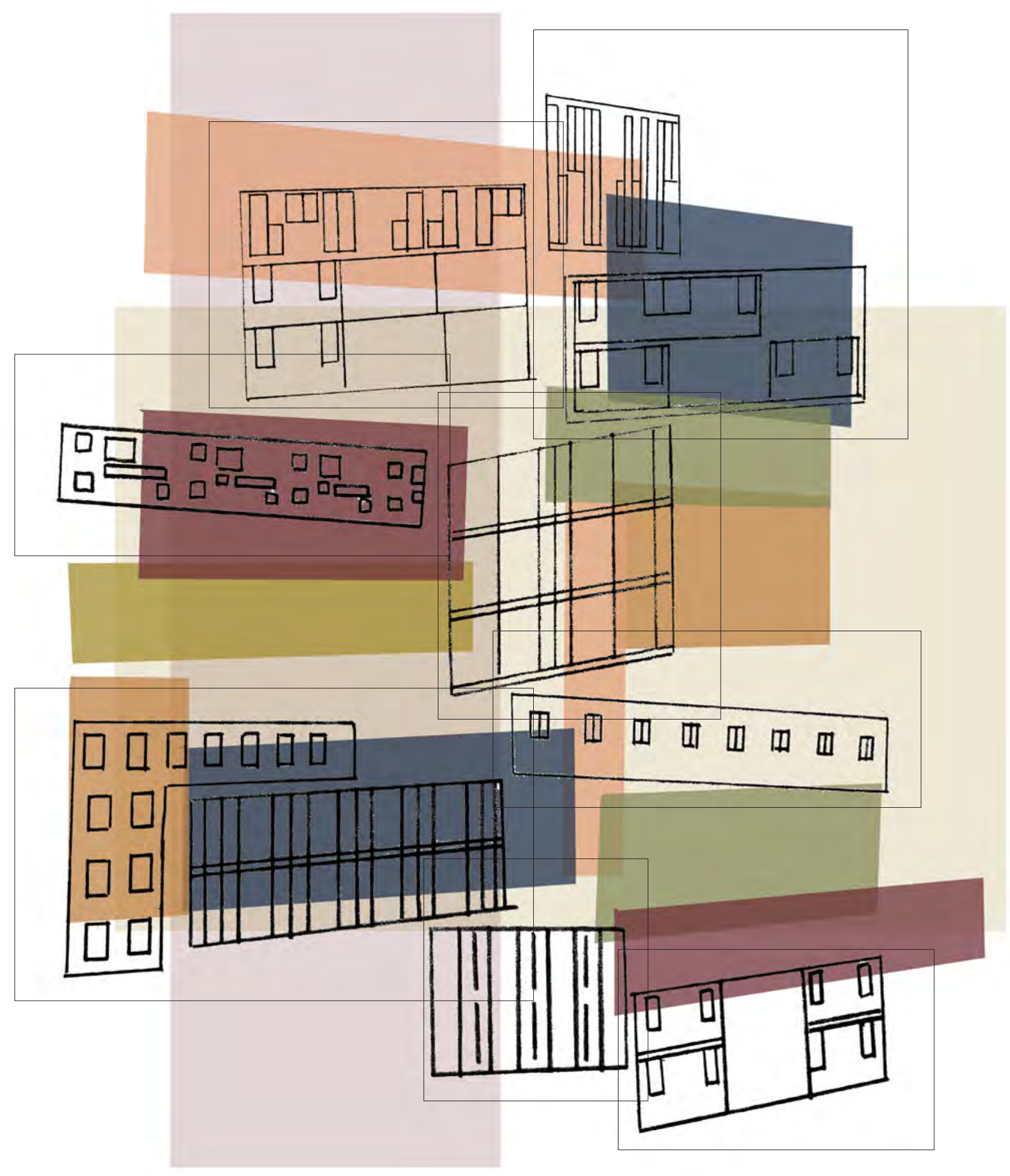




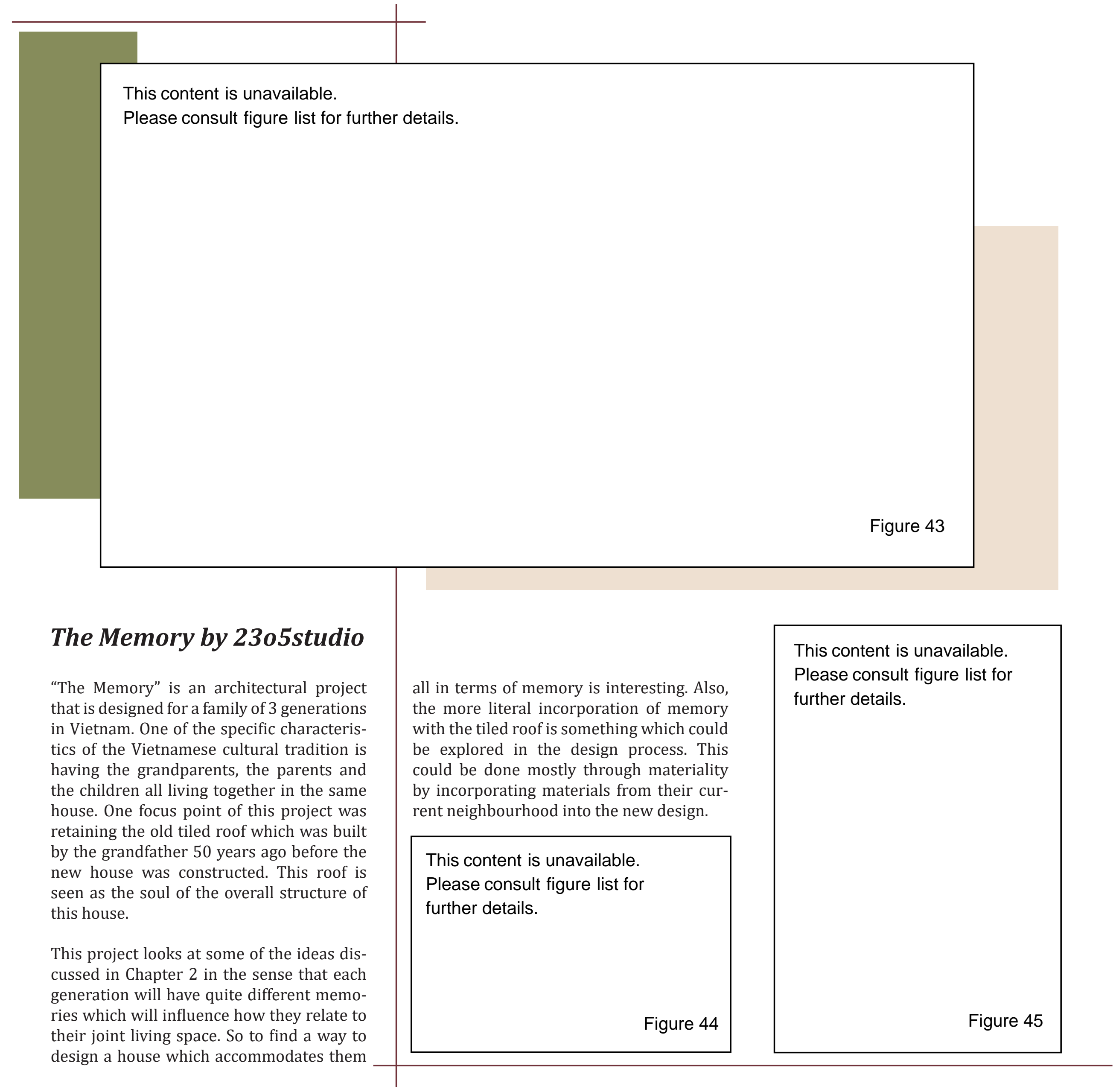




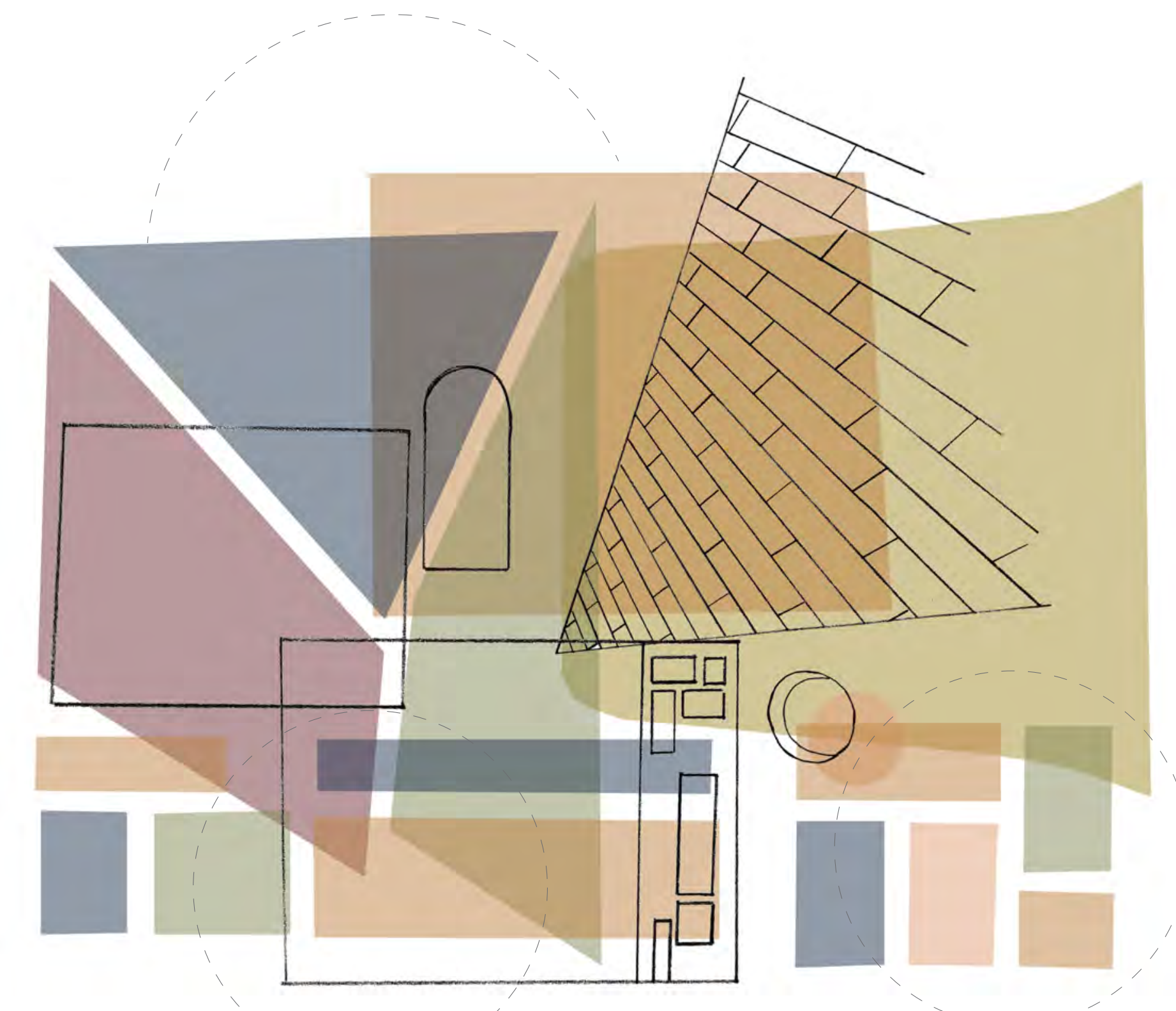




\section{Blaj Cultural Palace Refurbishment} by Vlad Sebastian Rusu

The Cultural Palace was designed in the year 1930 by the Bucharest architect Victor Smigelschi, and its primary purpose was to host ASTRA Cultural Association's events. In the winter of 1995 a violent fire burnt down most of the building, severely damaging the roof and the interior space of the main hall. It remained a ruin until 2012, when the municipality initiated a project for the rehabilitation and re-functionalisation of the Palace. The new design follows two directions: firstly, to evoke the recent tragic history of the building and secondly, to create a flexible interior and exterior space that can easily be adapted to the needs of the community that it serves.

This is a great example of how adaptive re-use can play with the idea of memory and new identity for both the building and the community. Incorporating elements of the old architecture as a nod to the old but also to embellish and support the new.

This content in unavailable.

Please consult figure list for further details.
This content in unavailable.

Please consult figure list for further details.
This content in unavailable.

Please consult figure list for further details.

Figure 46 


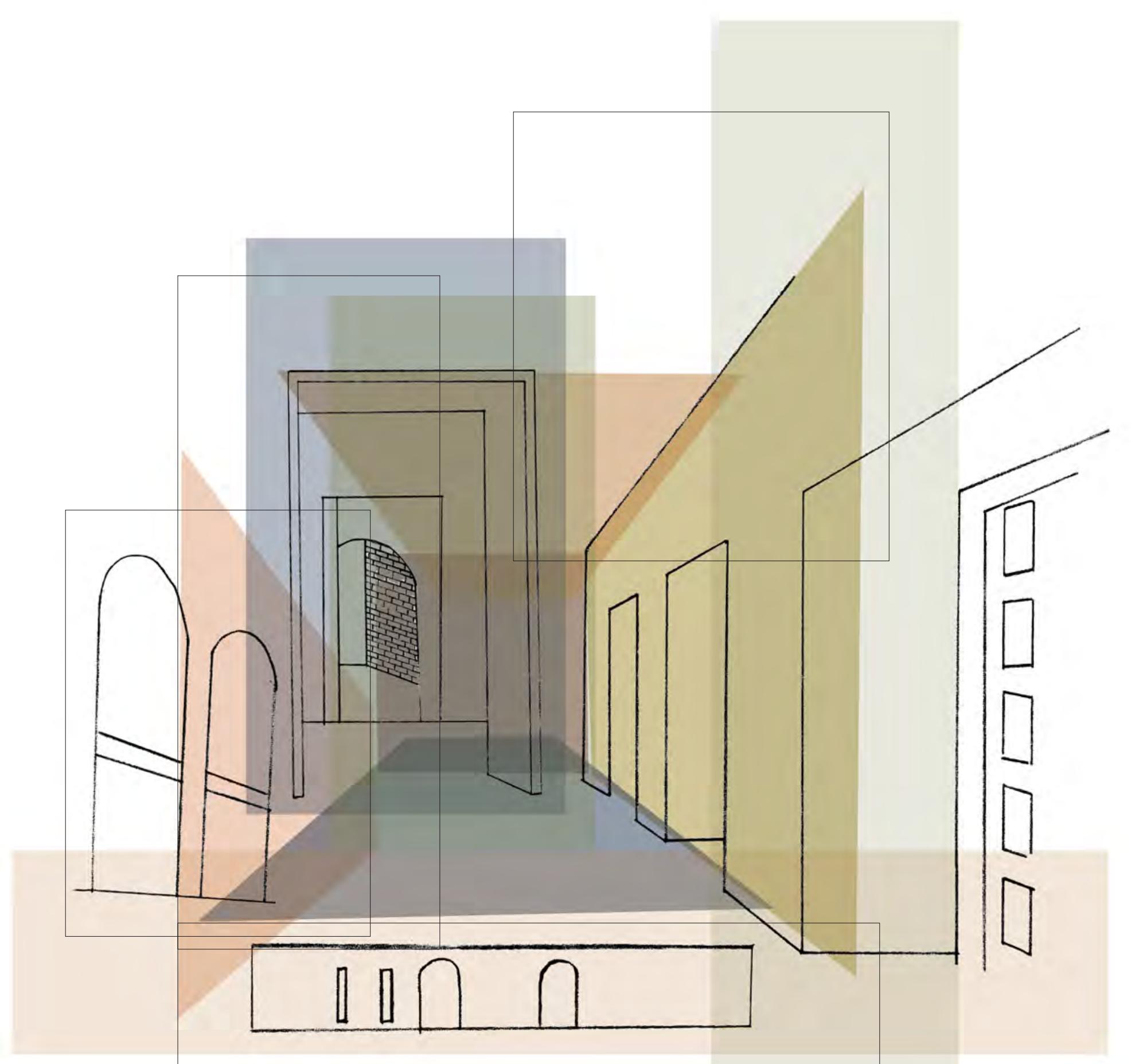




\section{Olivetti Showroom by Carlo Scarpa}

Designed in 1957-58, with construction complete in 1959, the showroom housed the Olivetti modern typewriters and calculator collections, made in the Italian town of Ivrea. Known for his stylisation of concrete and the expression of form, this showroom is no exception. The first encounter as you enter is a series of concrete platforms which are aligned together to form the central staircase. The use of stratification, layering and purposed offsets are evident throughout. The hand-cut mosaic floors, concrete, stone, bodies of water and beautifully polished timbered nods all come together to create a harmonious interior architecture.

Scarpa's work brings together a vast range of materiality and details to create one interior architecture. This showroom shows clear evidence of this. Scarpa's work becomes a good example for not only engaging the visual and tactile sensory experiences in the form of materiality and detailing which will be important for this design-led research but it also begins to evoke the idea of layering in a much subtler way.

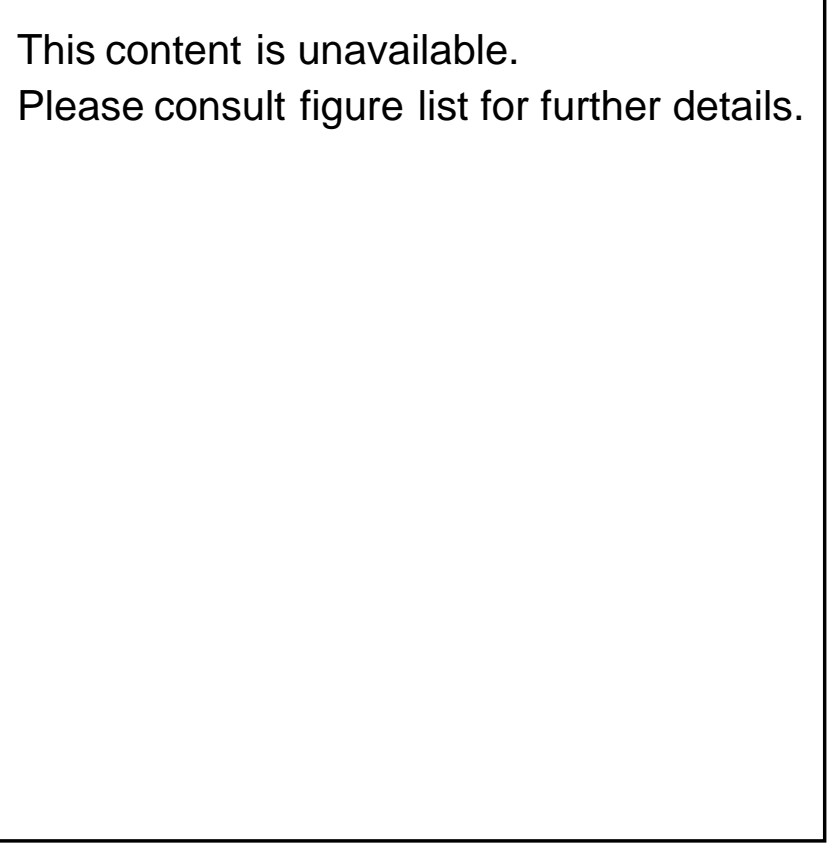

This content is unavailable.

Please consult figure list for further details.
This content is unavailable.

Please consult figure list for further details.
This content is unavailable.

Please consult figure list for further details. 


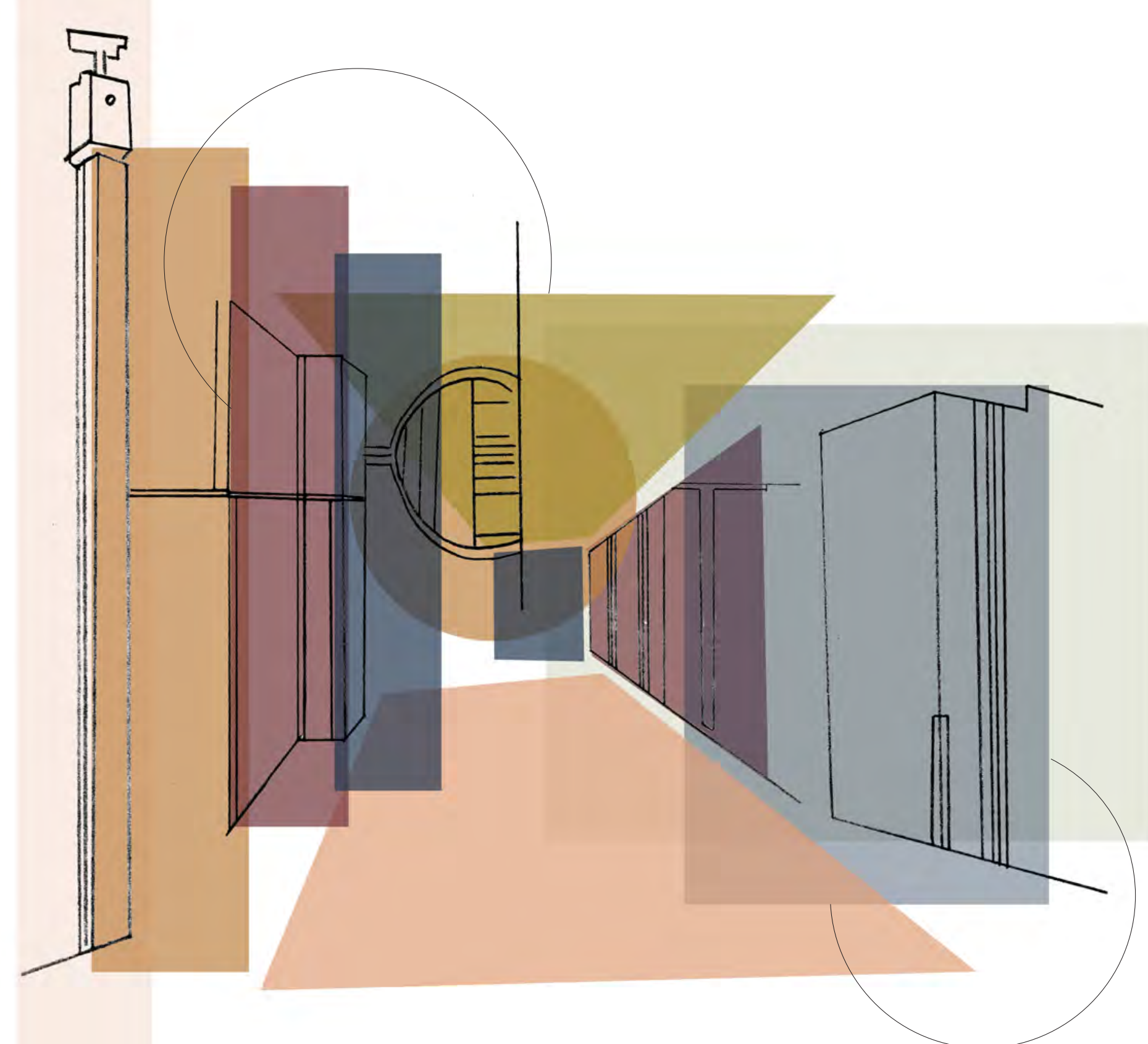




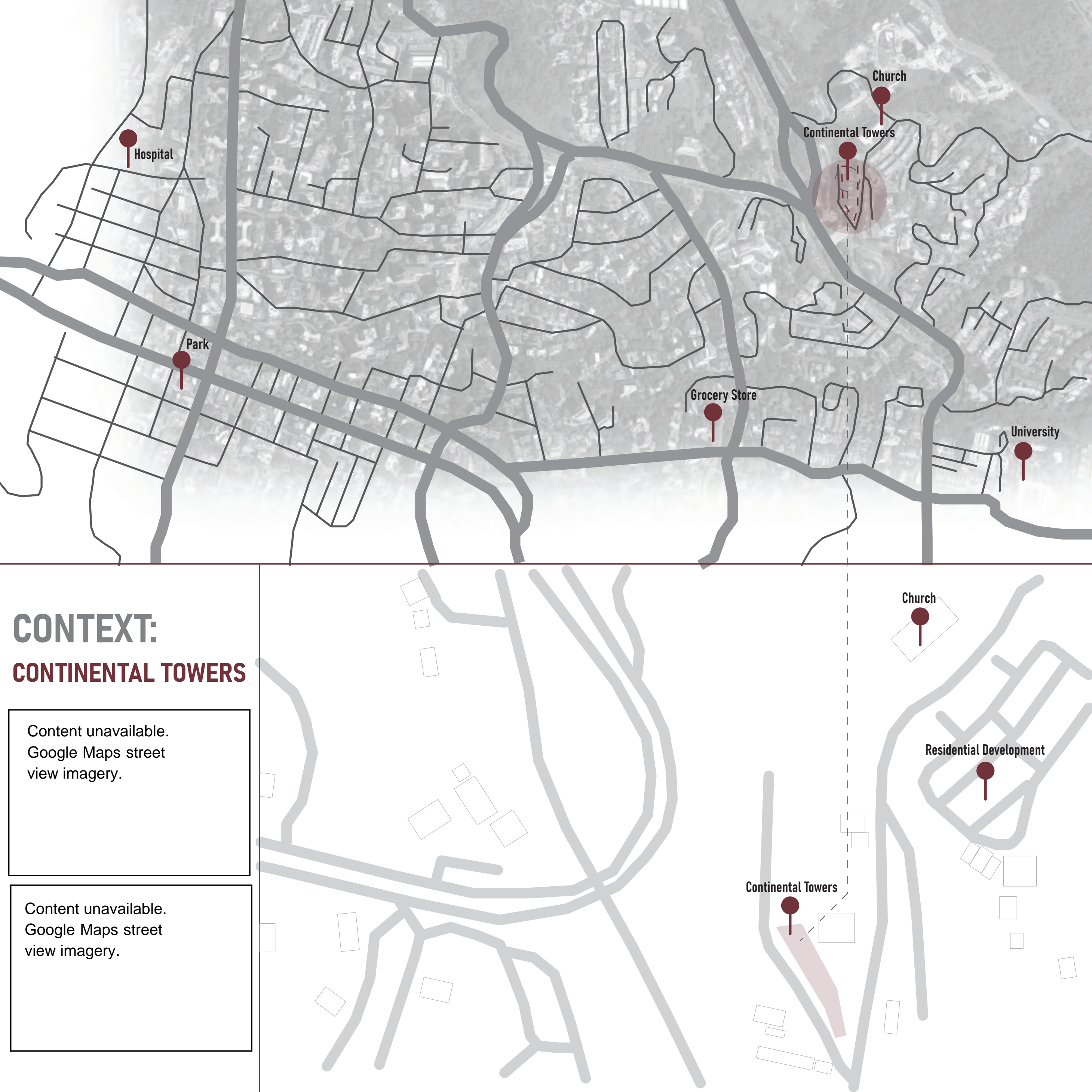




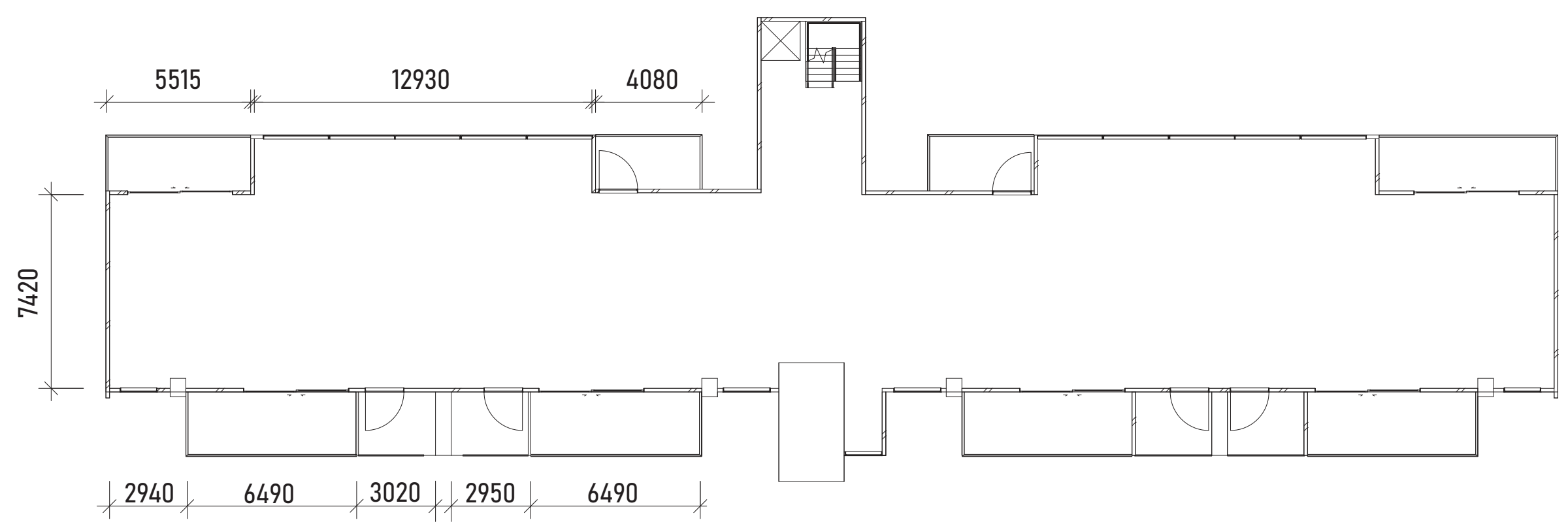

Existing Building Floor Plan - Level 1

1: 250

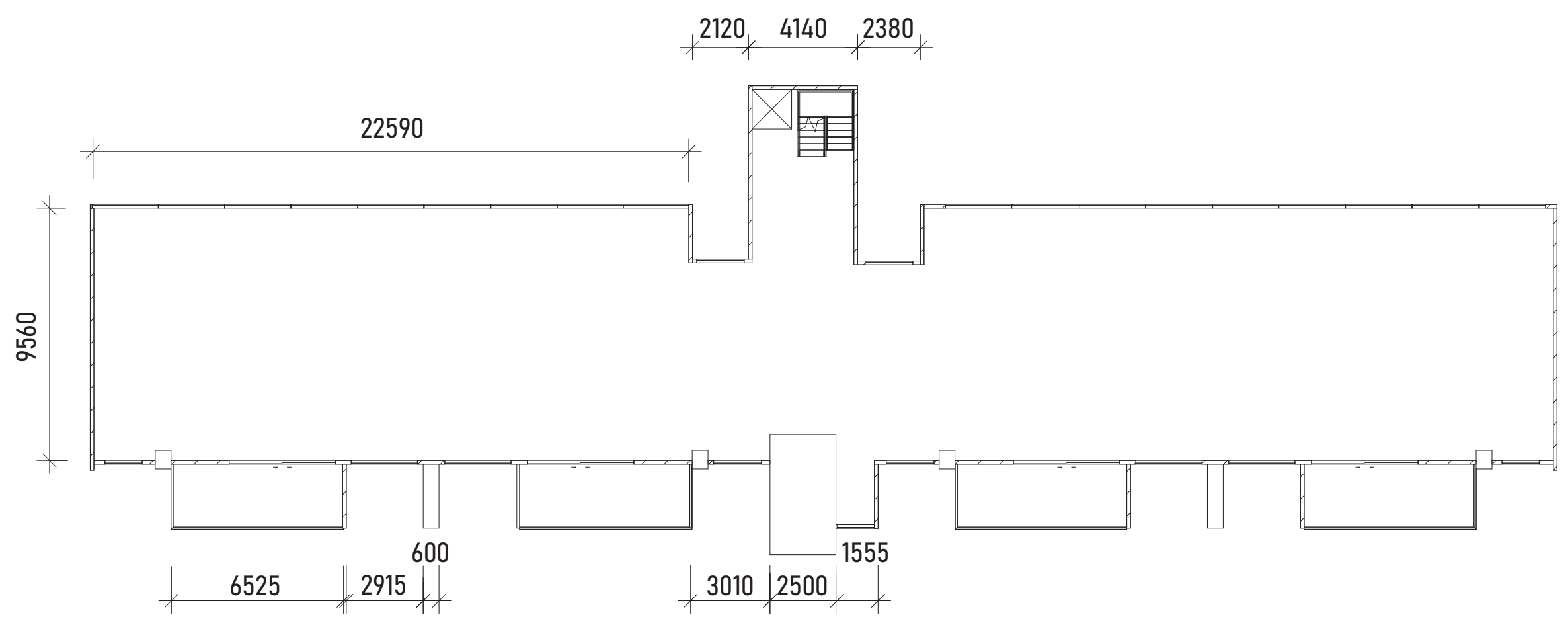

Existing Building Floor Plan - Level 2

1: 250

This is an abandoned building known as Continental Towers located quite close to the city center.

This site was selected precisely for its proximity to the city centre which aligns with the theories on high-density housing that were previously mentioned of placing the community closer to the wider city and education and job possibilities.

The interior of the building consists of mostly concrete floors and walls. It also has low ceilings and existing access to balconies. 
THE BEGINING OF FLOOR PLANS

Iteration 1
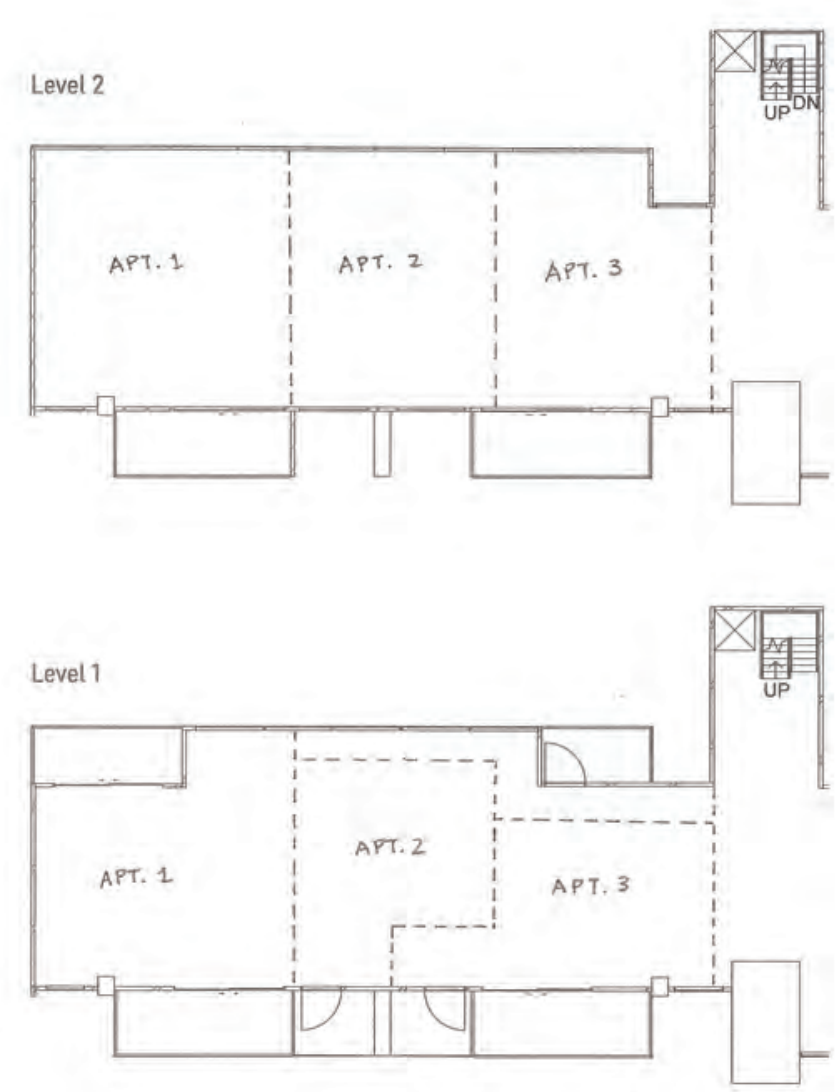

Iteration 3
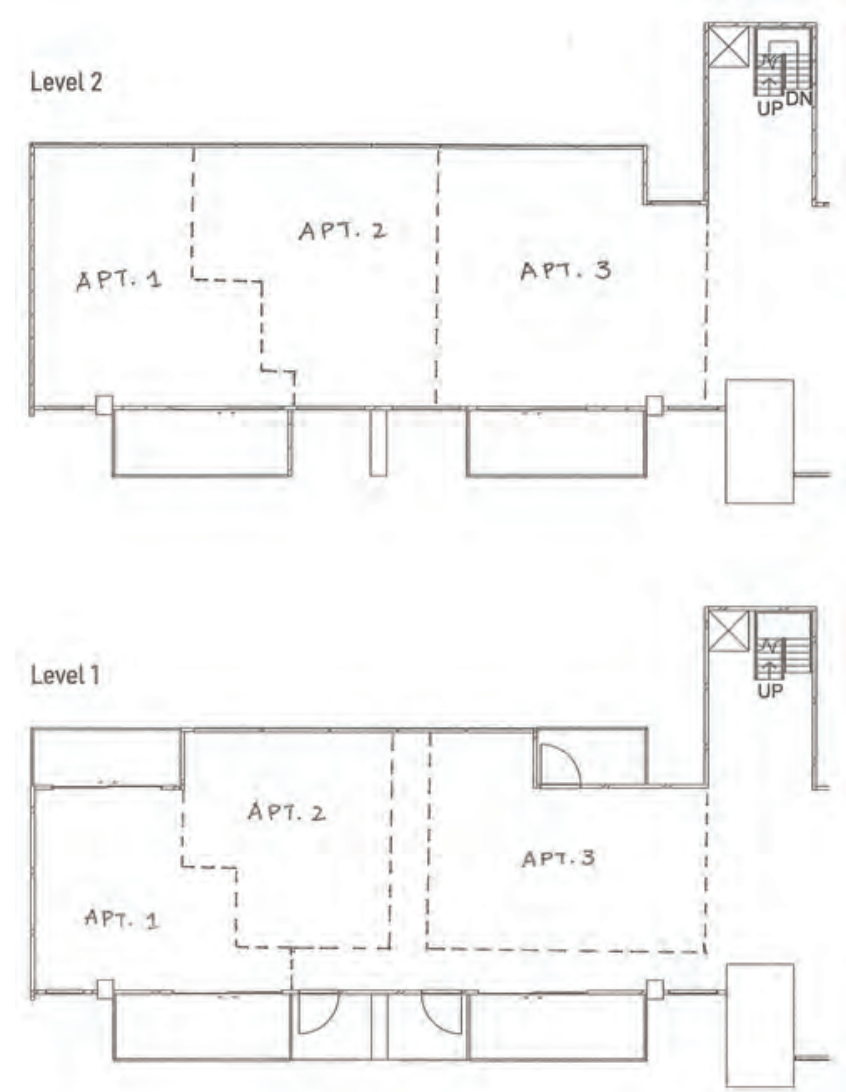

Iteration 2
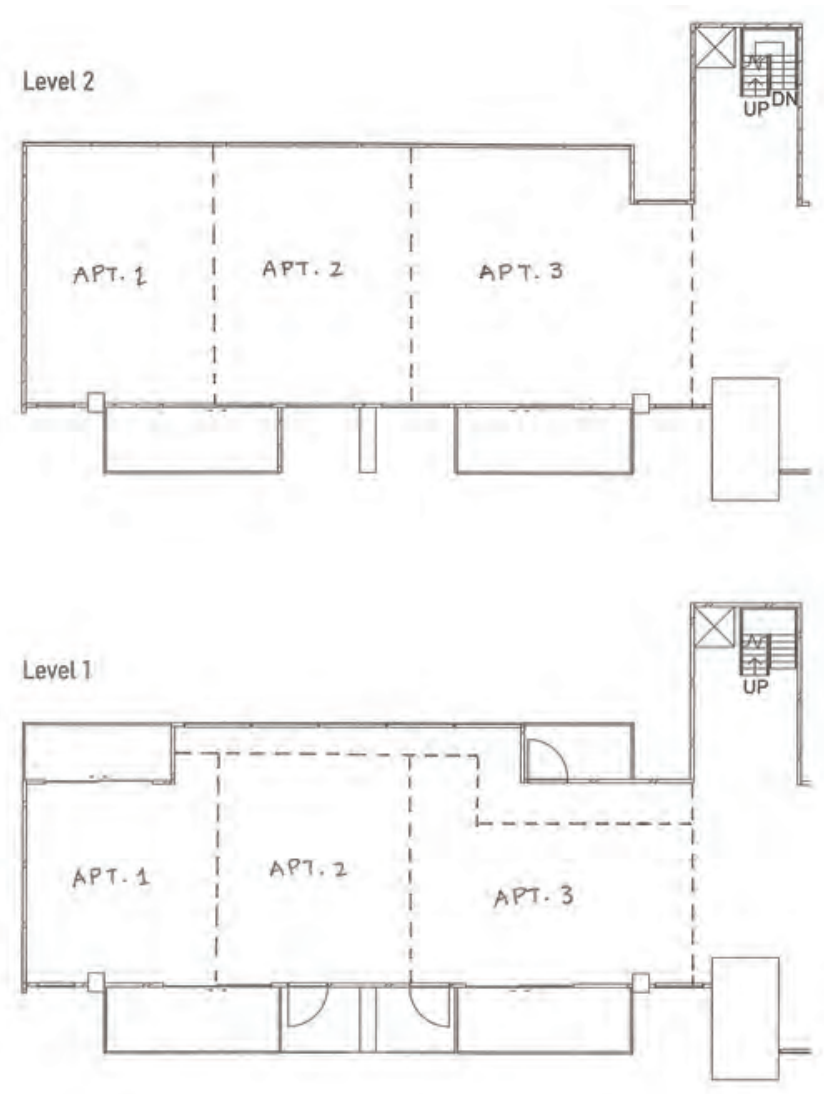

Iteration 4
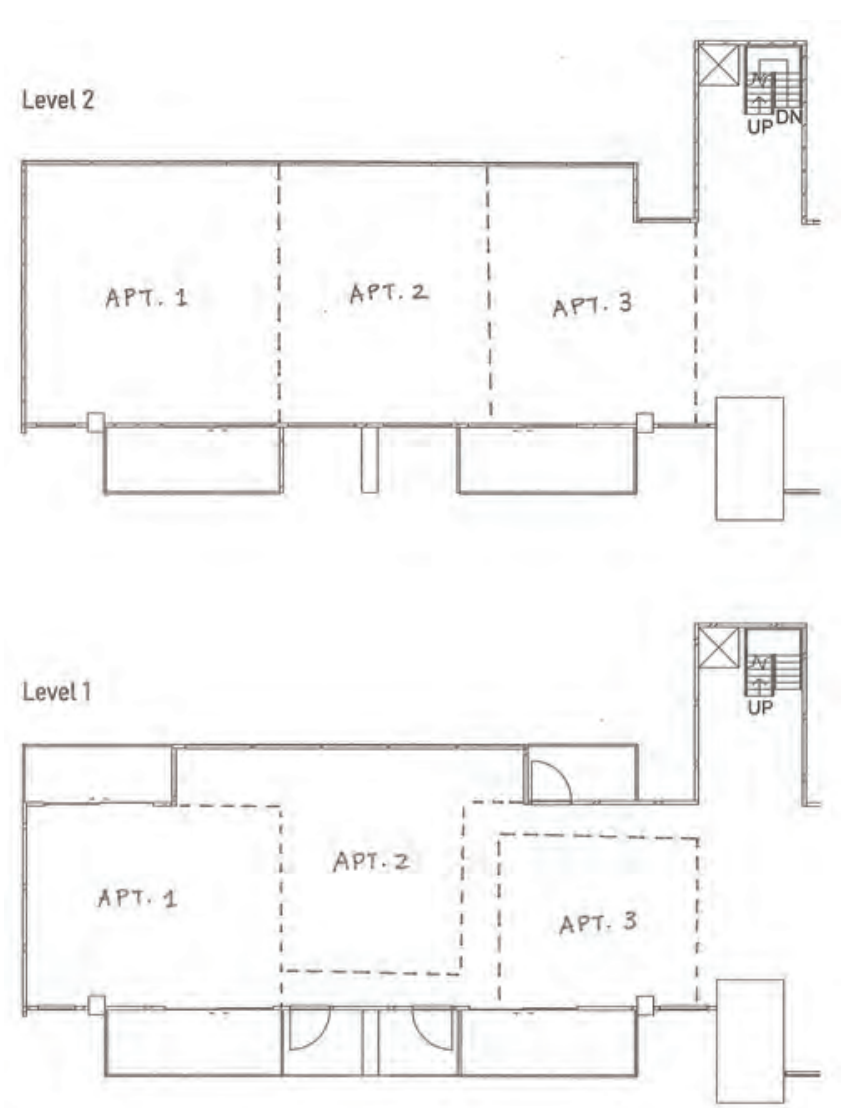
THE AREAS

Establishing the minimum area requirements for the rooms within the apartments
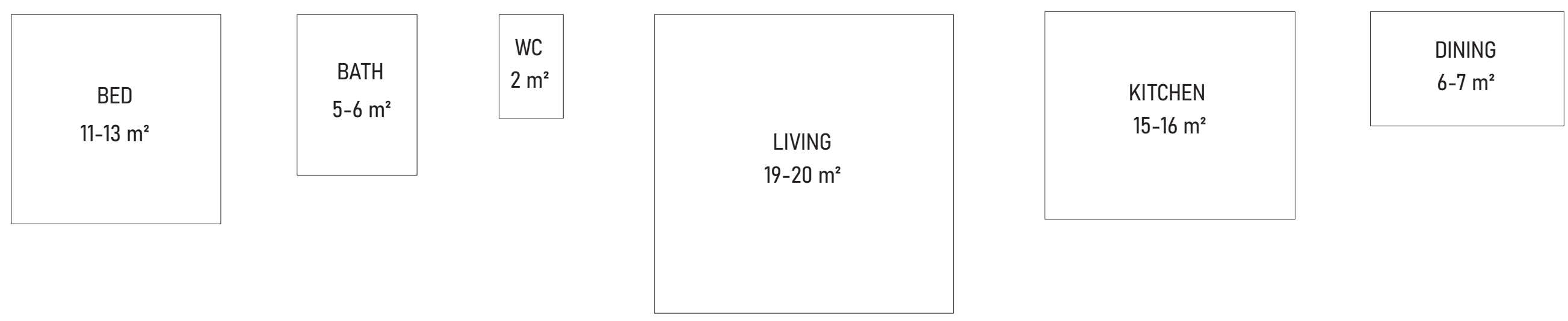

The following is a diagrammatic display of variations of these areas and how furniture/fixtures could fit within them to gain a further understanding of the spaces being created.
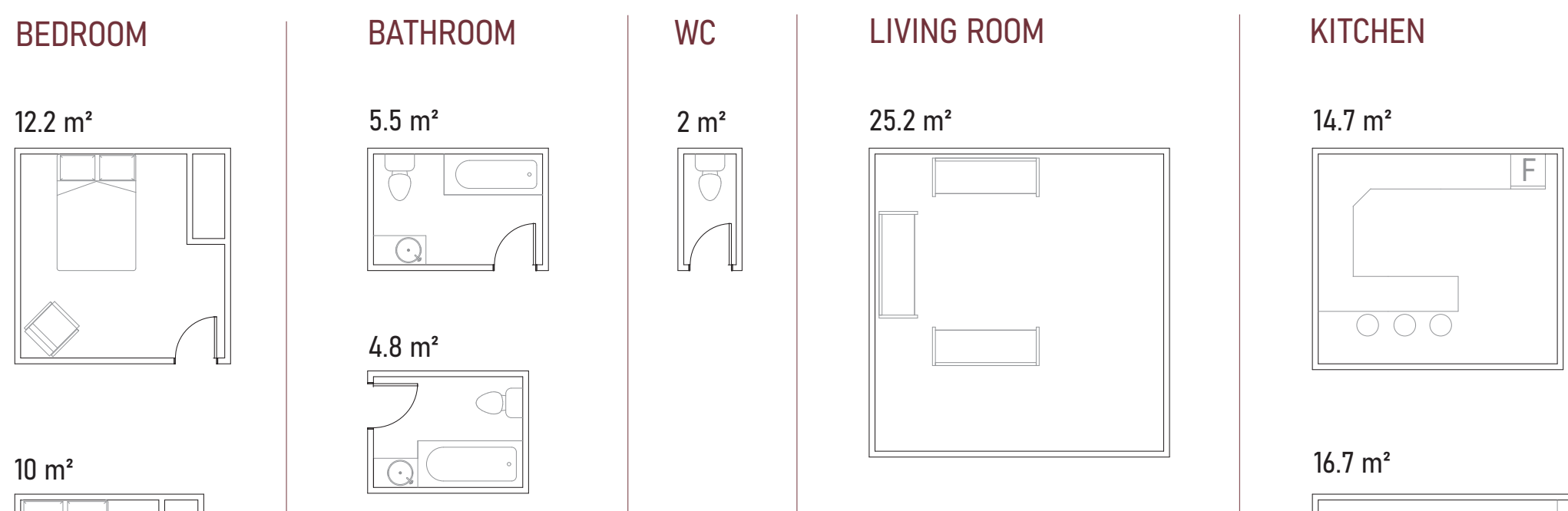

DINING
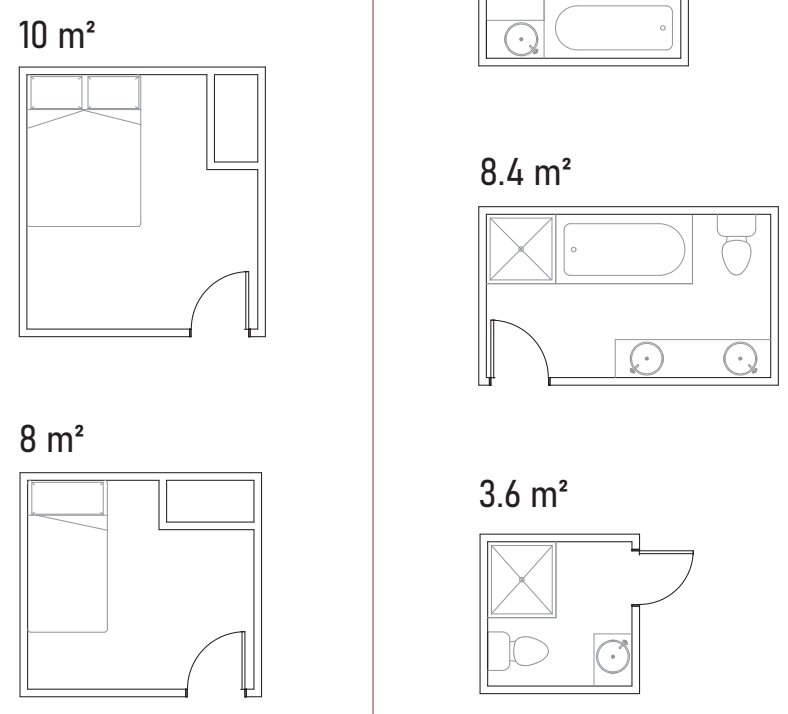

$3.6 \mathrm{~m}^{2}$

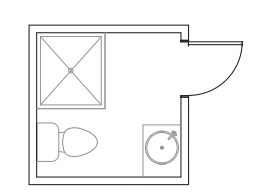

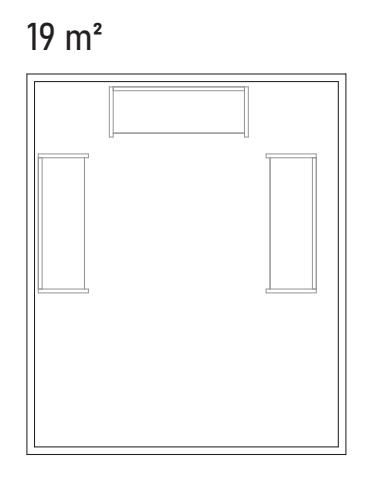

\section{$16.7 \mathrm{~m}^{2}$}

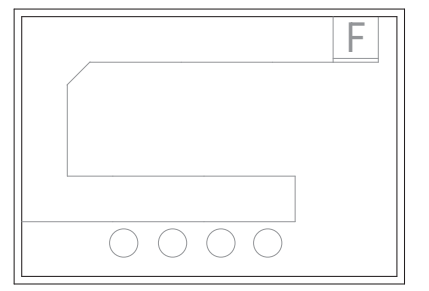

$5.6 \mathrm{~m}^{2}$

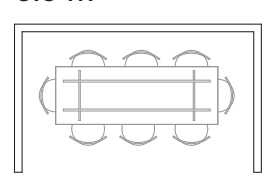

$7.9 \mathrm{~m}^{2}$

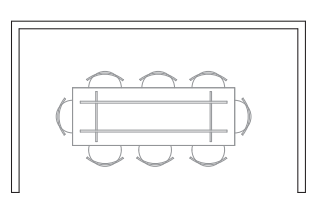

$10 \mathrm{~m}^{2}$

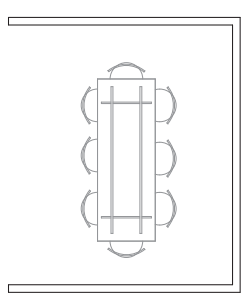

It is envisioned that the living, kitchen, and dining spaces will be open plan spaces and therefore their areas will merge at some points. 


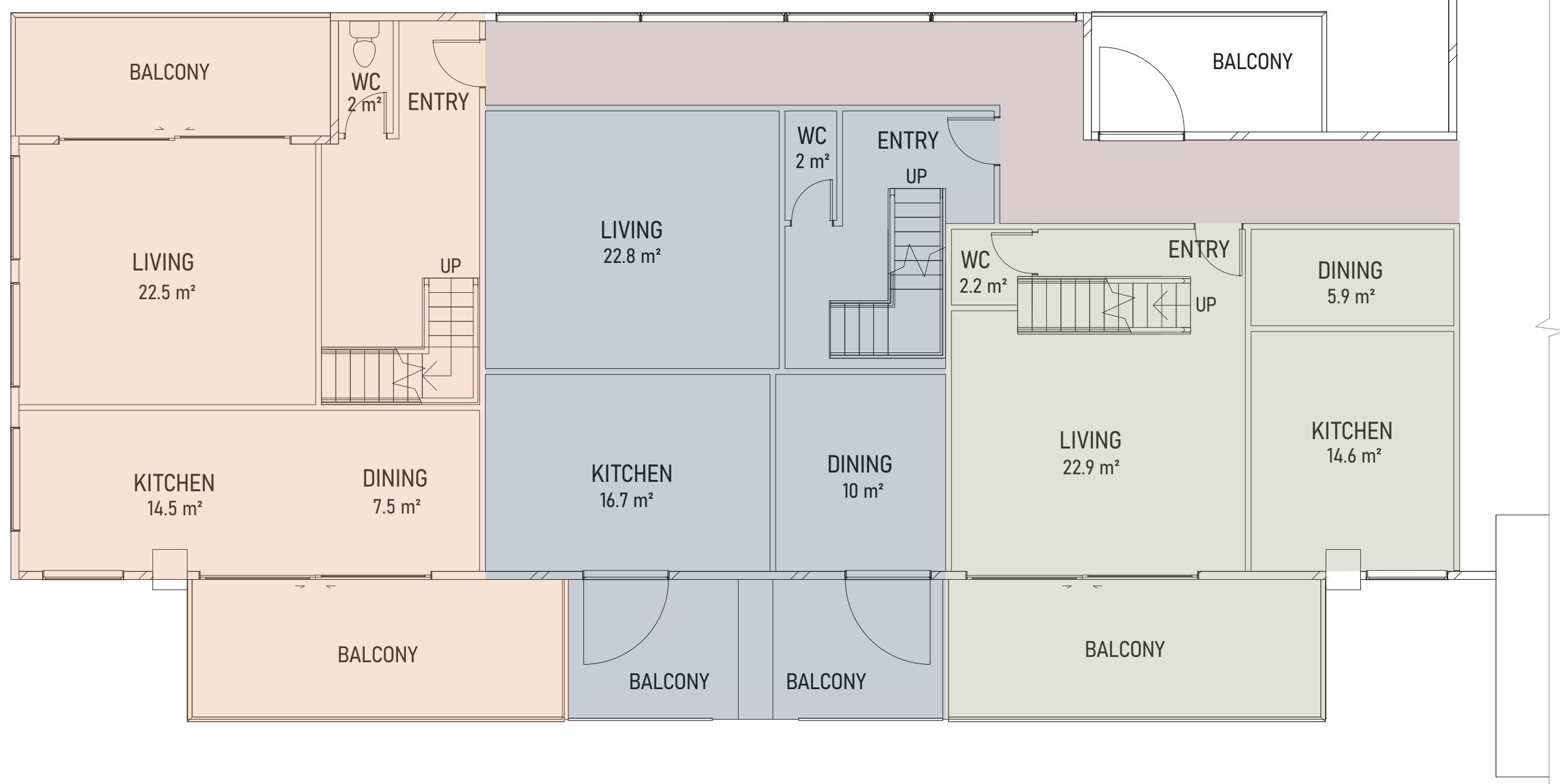

Floor Plan Iteration 1 - Level 1

$1: 100$ 
APARTMENT 1

APARTMENT 2

APARTMENT 3

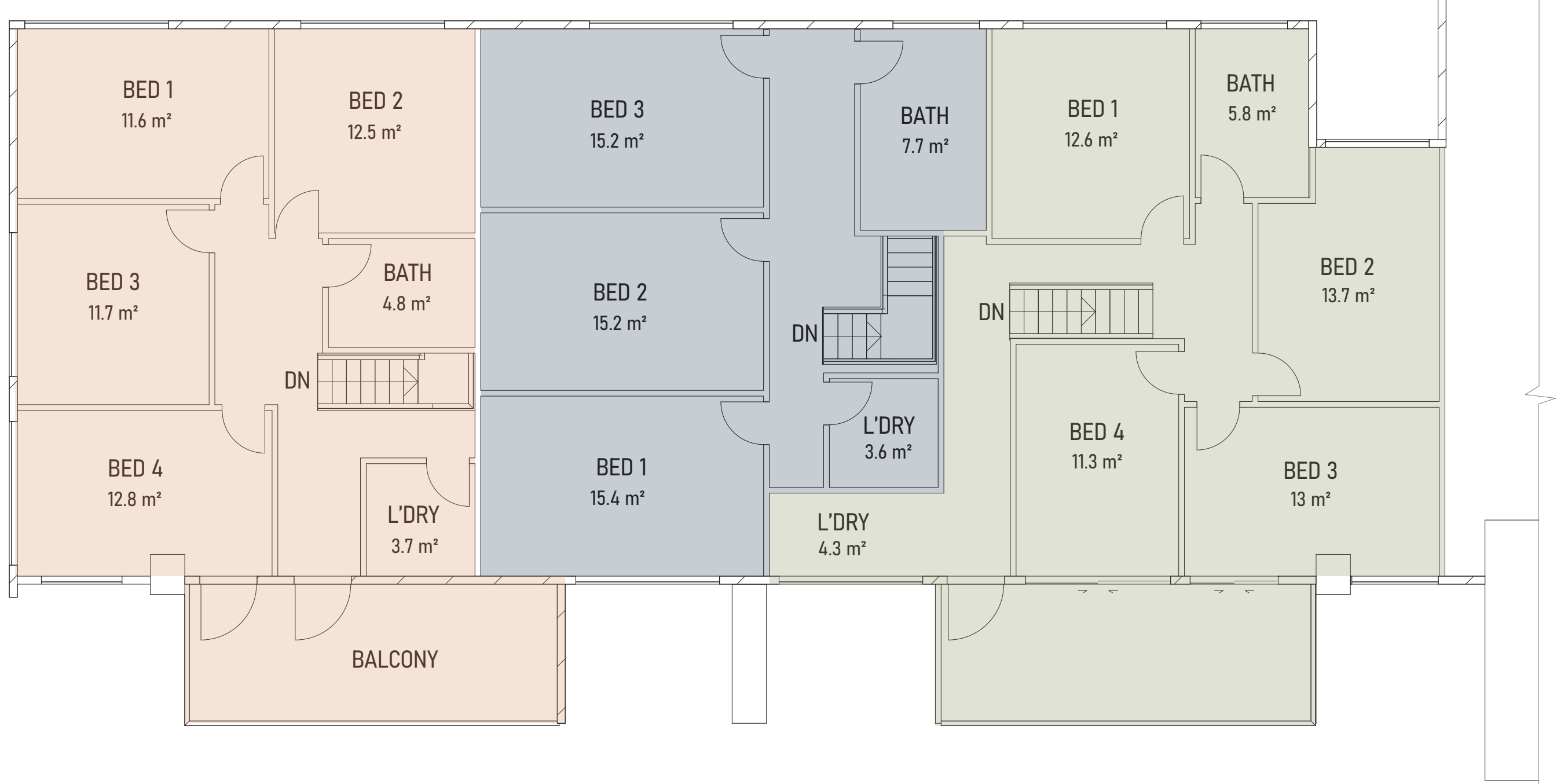

Floor Plan Iteration 1 - Level 2

1: 100 
APARTMENT 1

APARTMENT 2

APARTMENT 3

ACCESS

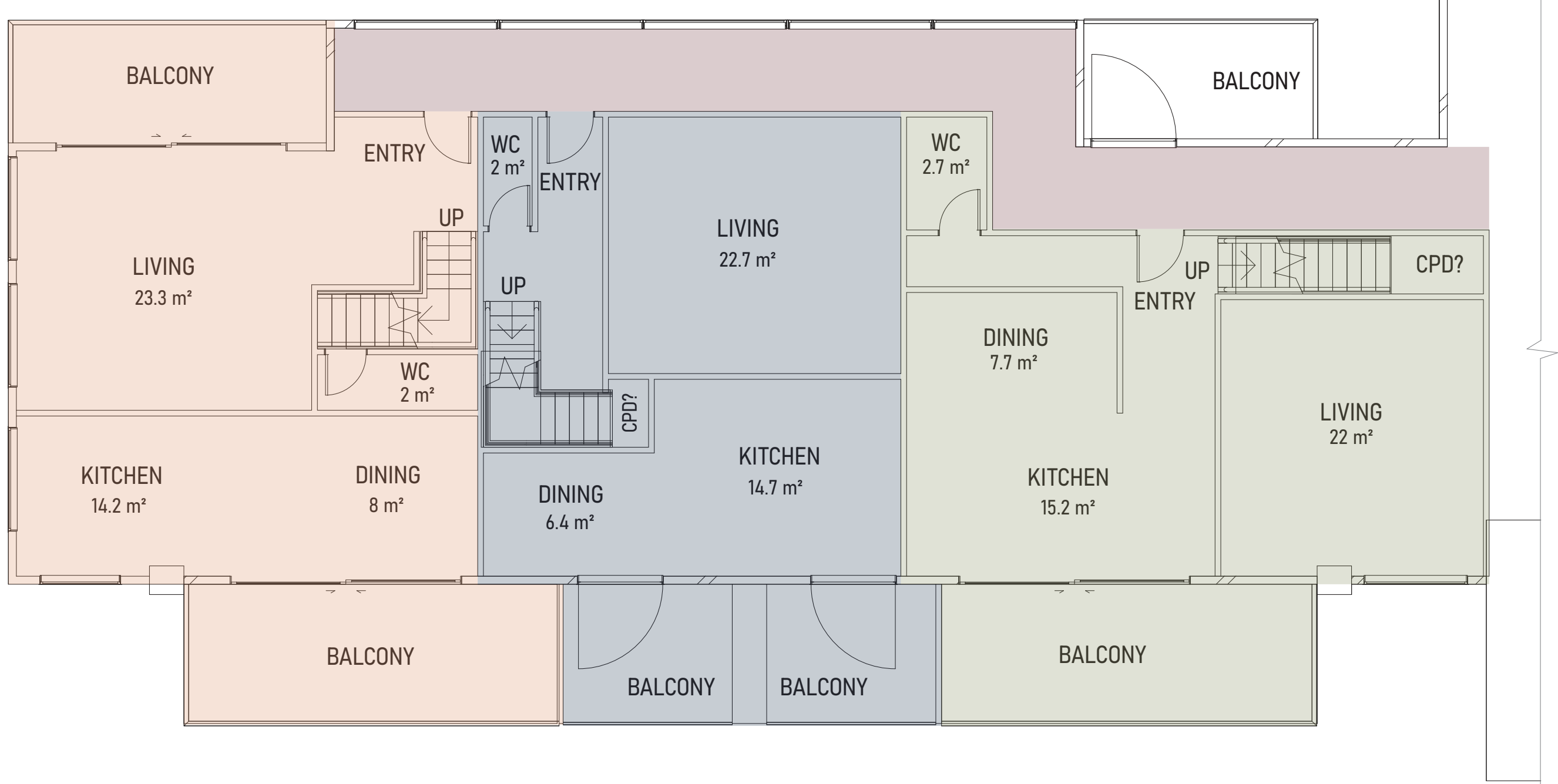

Floor Plan Iteration 2 - Level 1

$1: 100$ 
APARTMENT 1

APARTMENT 2

APARTMENT 3

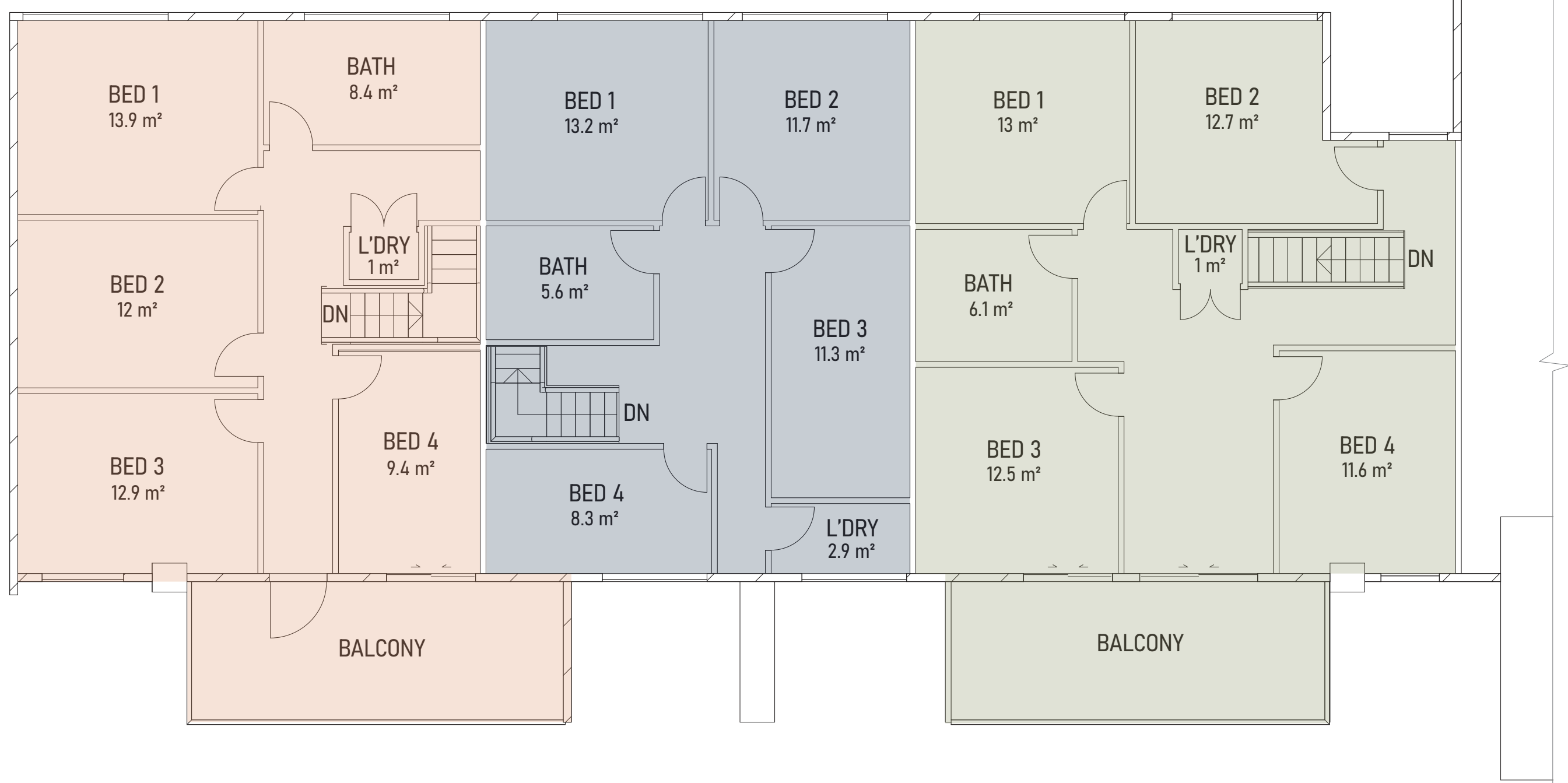

Floor Plan Iteration 2 - Level 2

$1: 100$ 


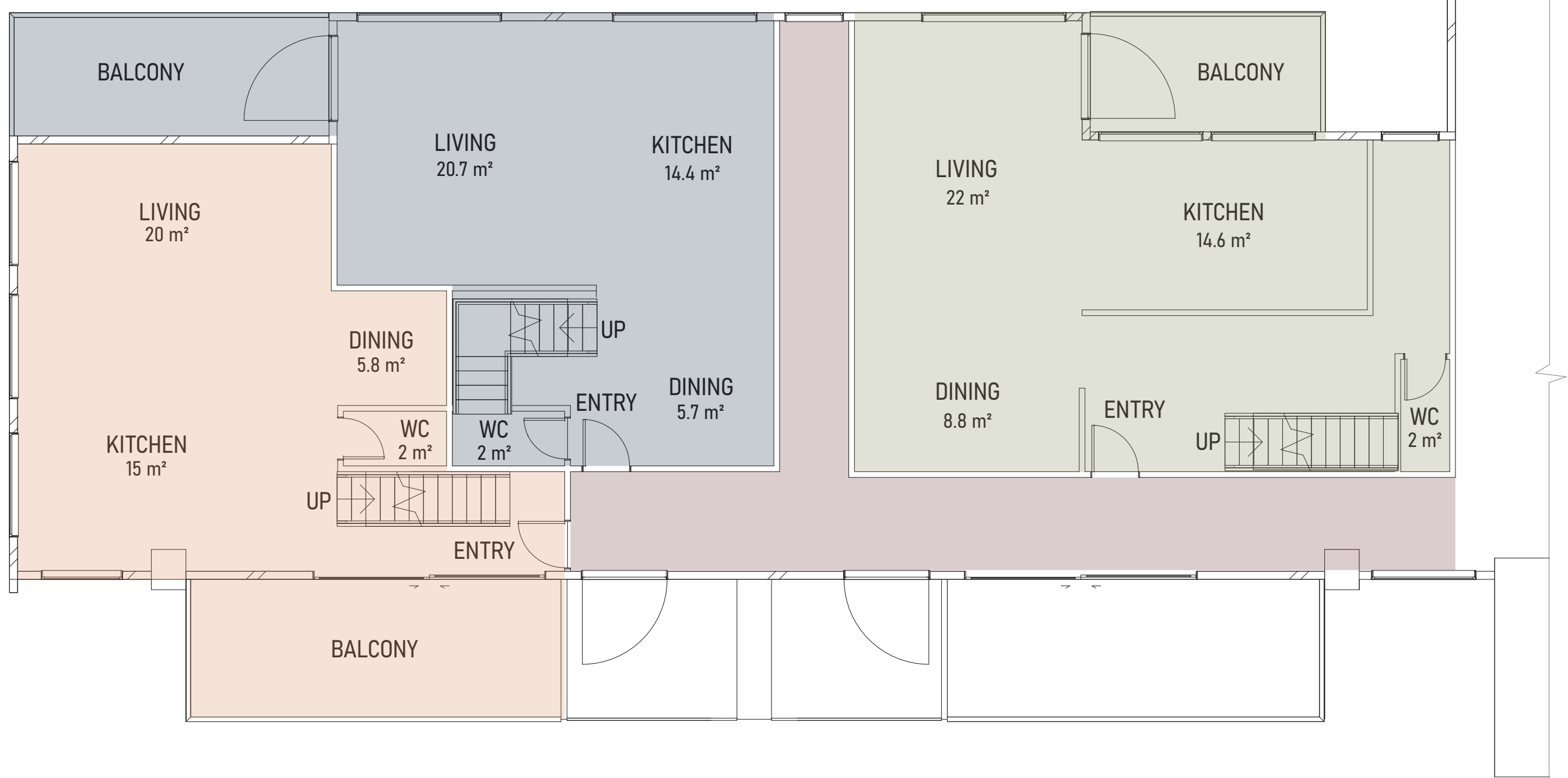

Floor Plan Iteration 3 - Level 1

$1: 100$ 


\section{APARTMENT 1}

APARTMENT 2

APARTMENT 3

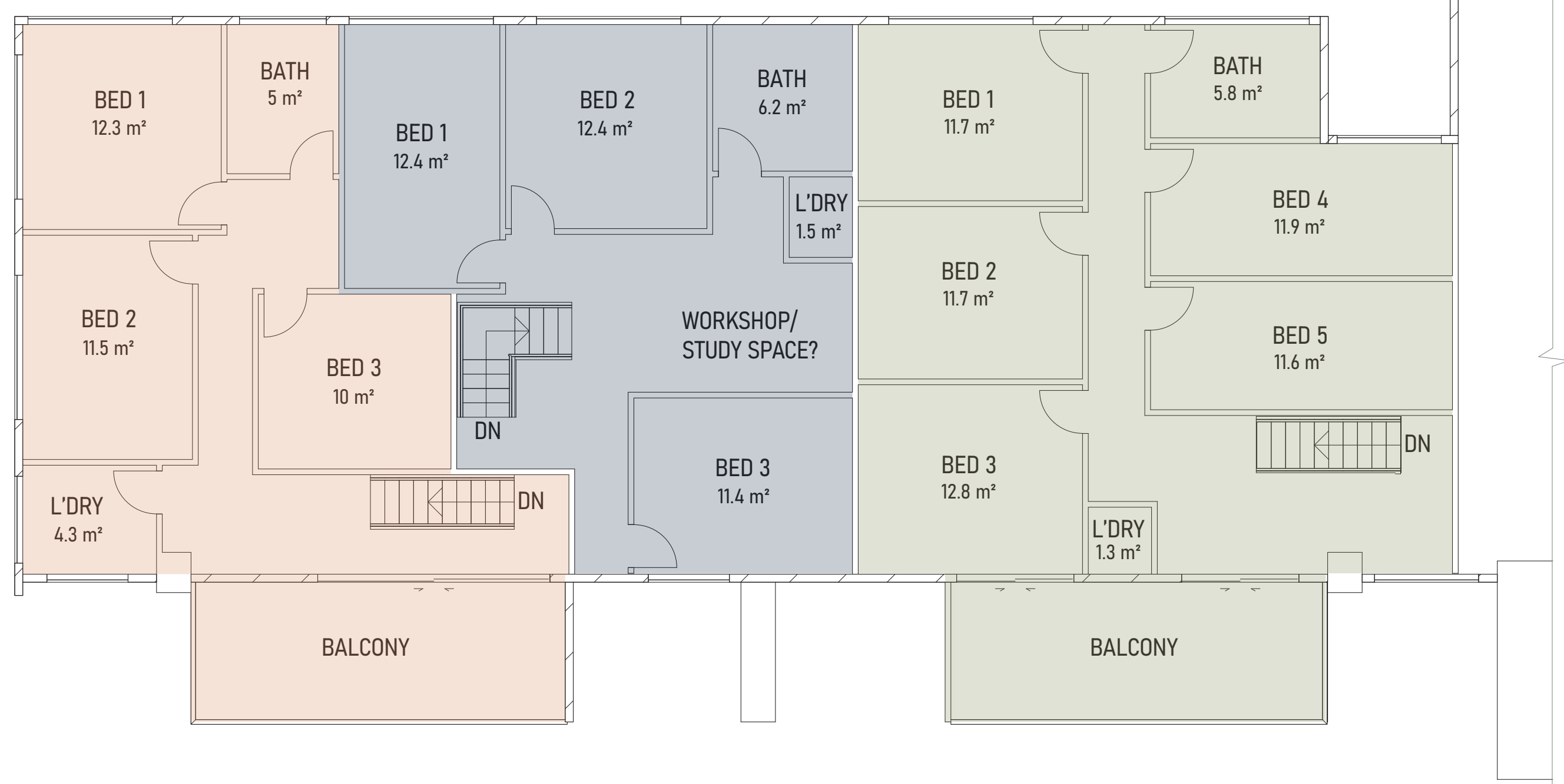

Floor Plan Iteration 3 - Level 2

$1: 100$ 


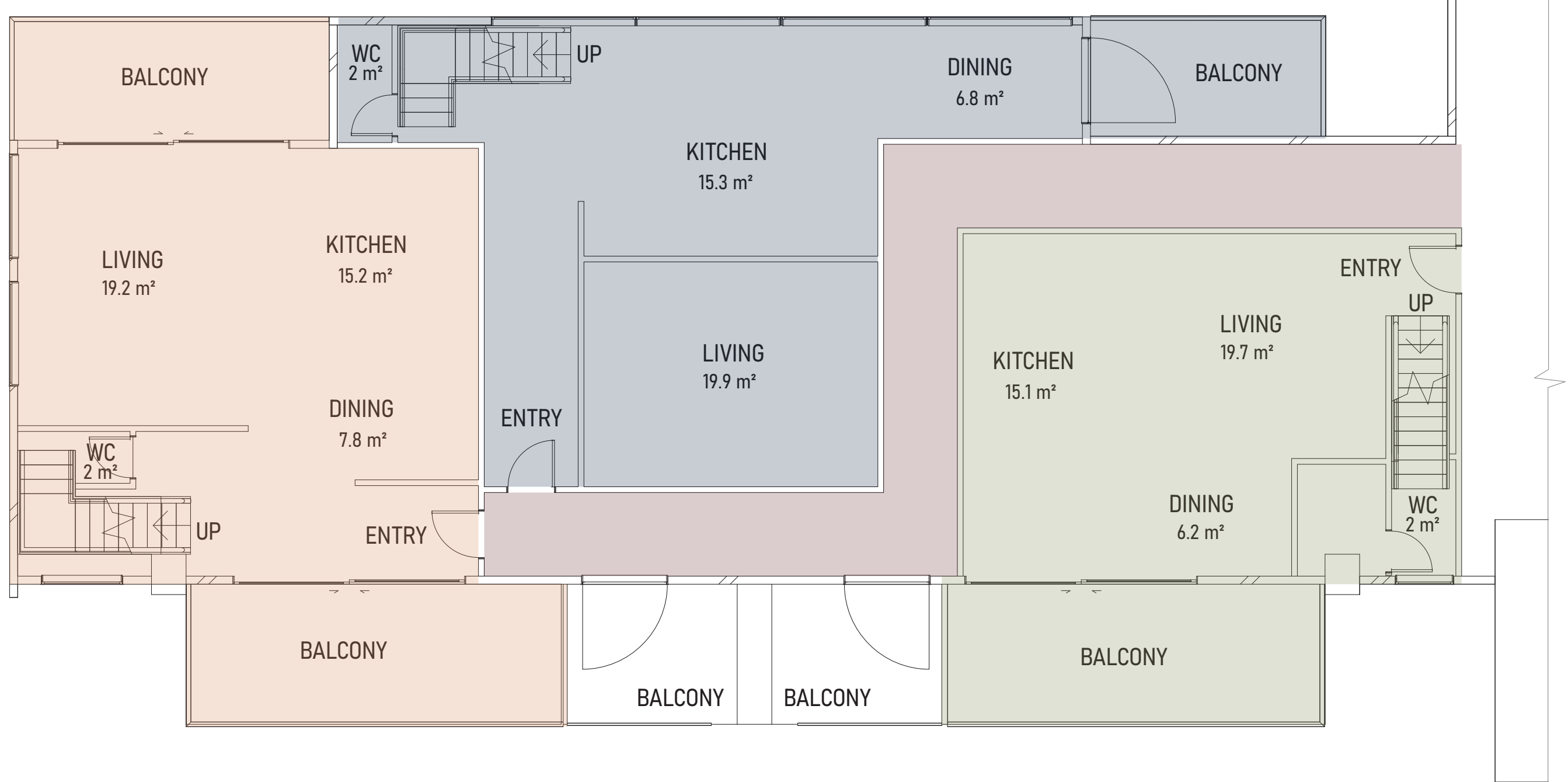

Floor Plan Iteration 4 - Level 1

$1: 100$ 
APARTMENT 2

APARTMENT 3

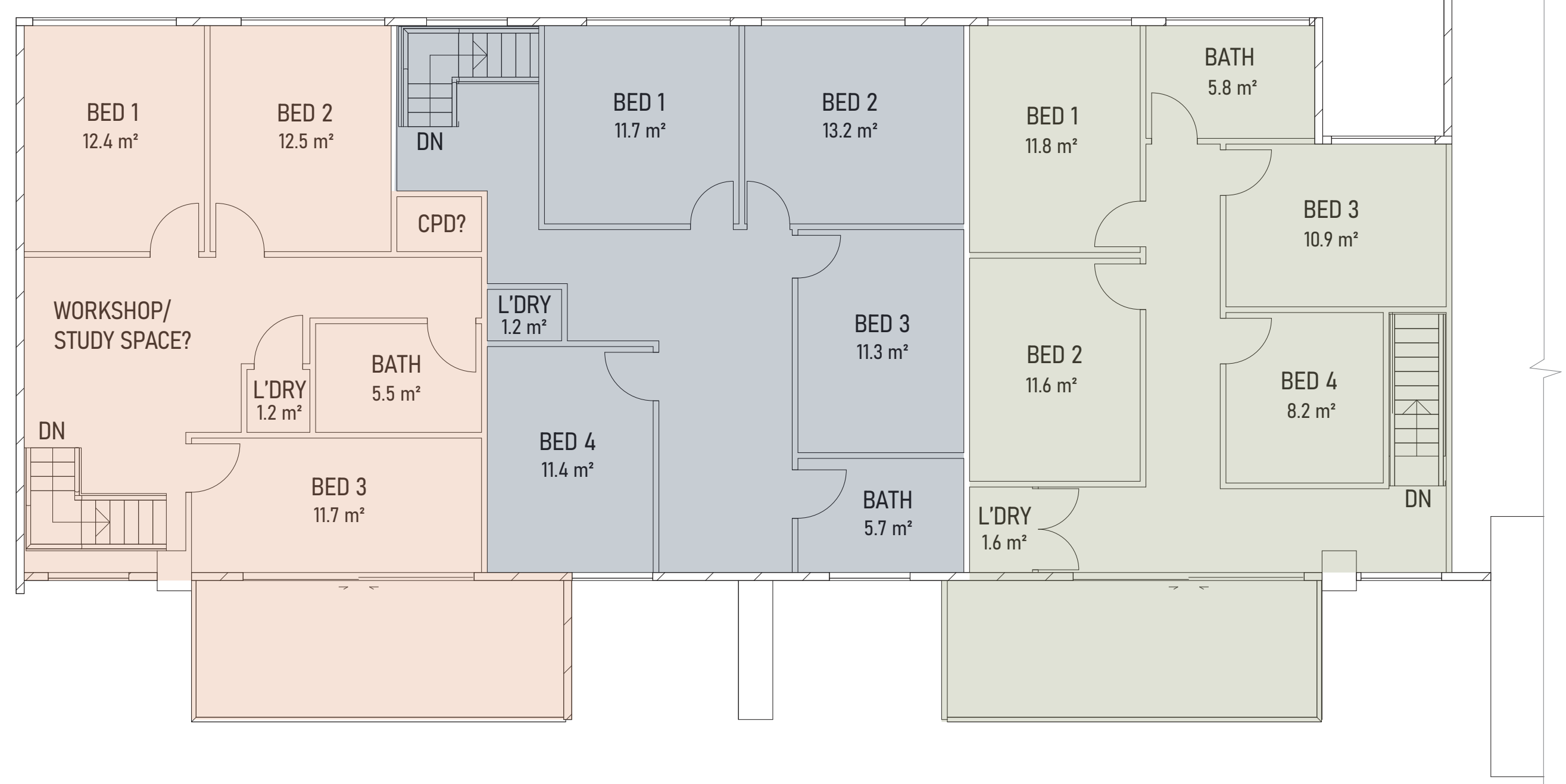

Floor Plan Iteration 4 - Level 2

$1: 100$ 
THE DISTURBANCE - PHASE 1

New walls

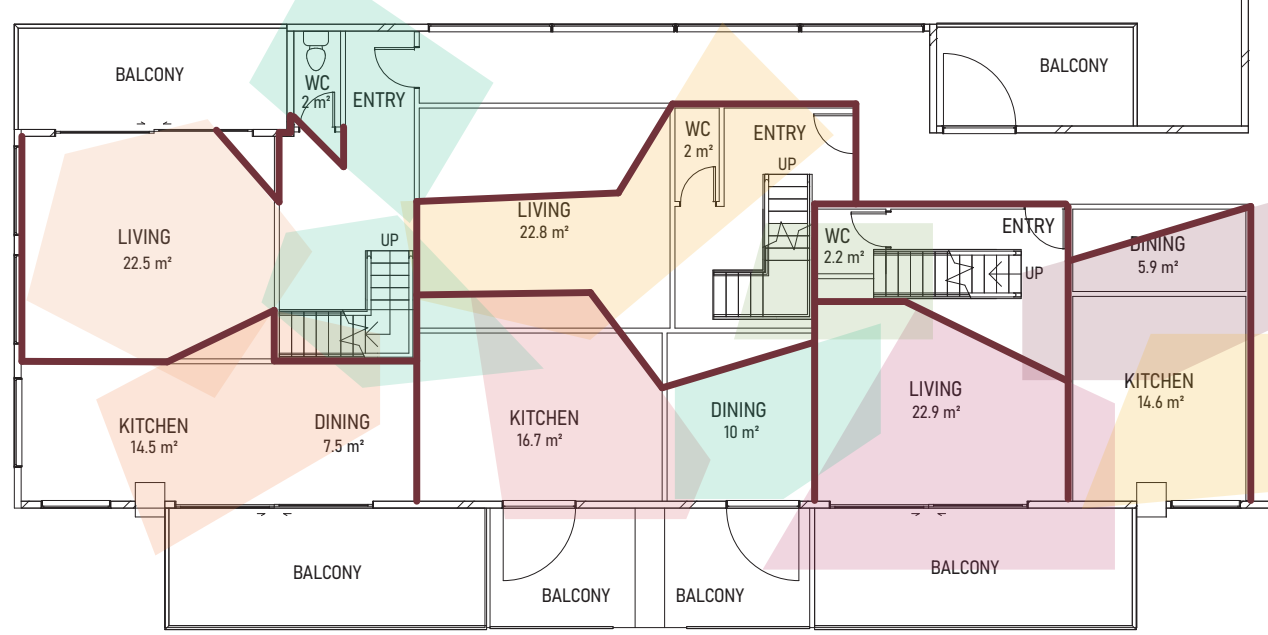

Floor Plan Iteration 1 - Level 1 $1: 200$
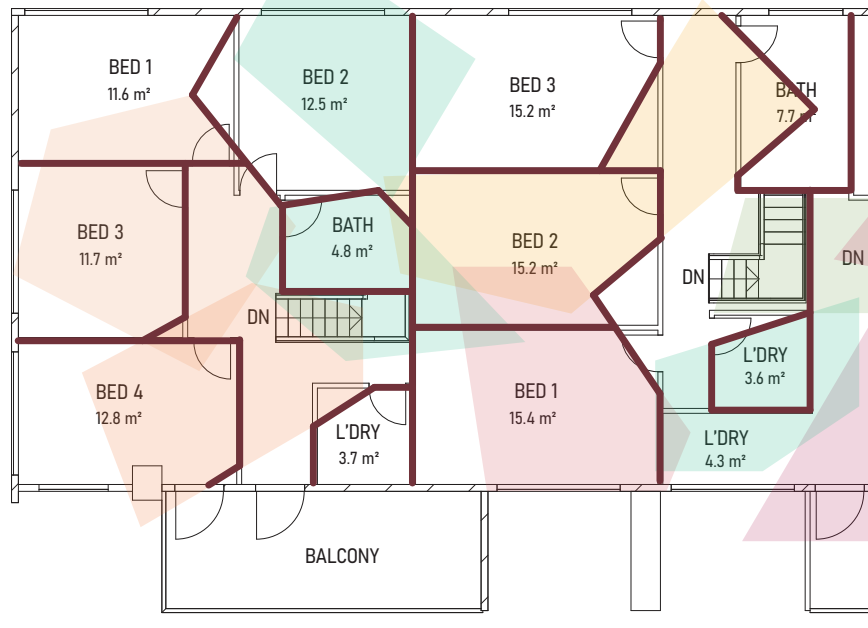

Floor Plan Iteration 1 - Level 2

$1: 200$

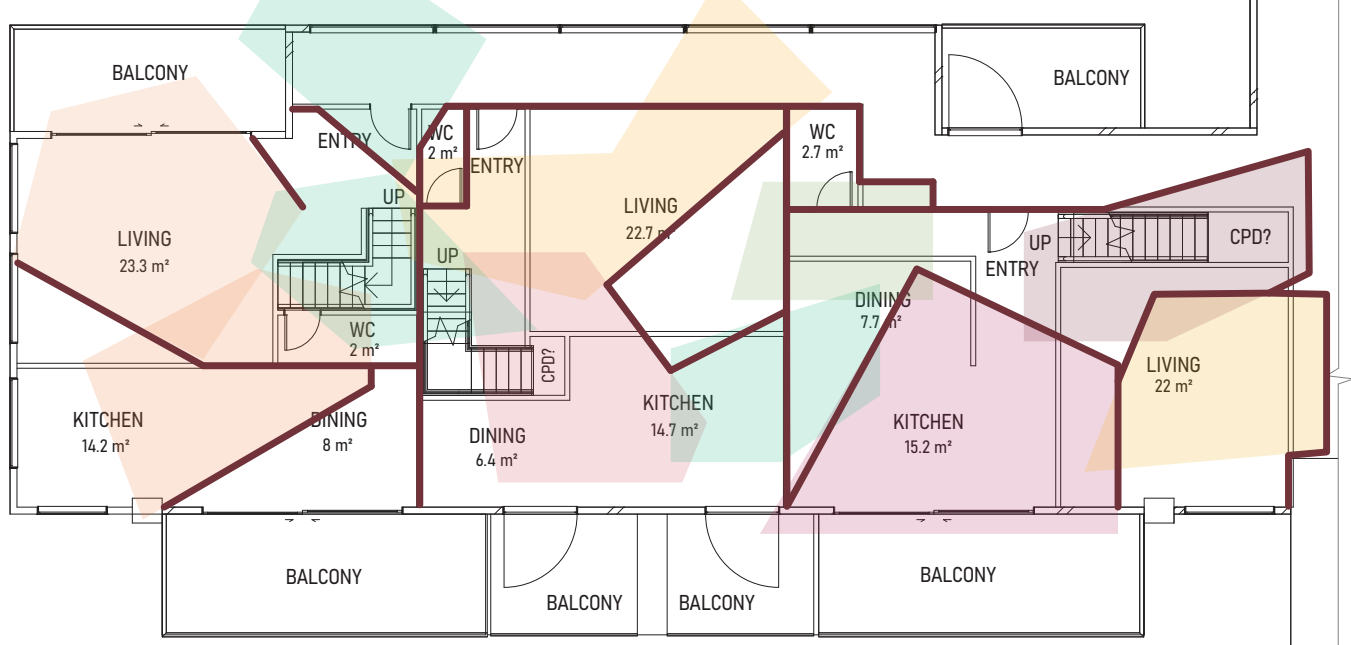

Floor Plan Iteration 2 - Level 1

1: 200

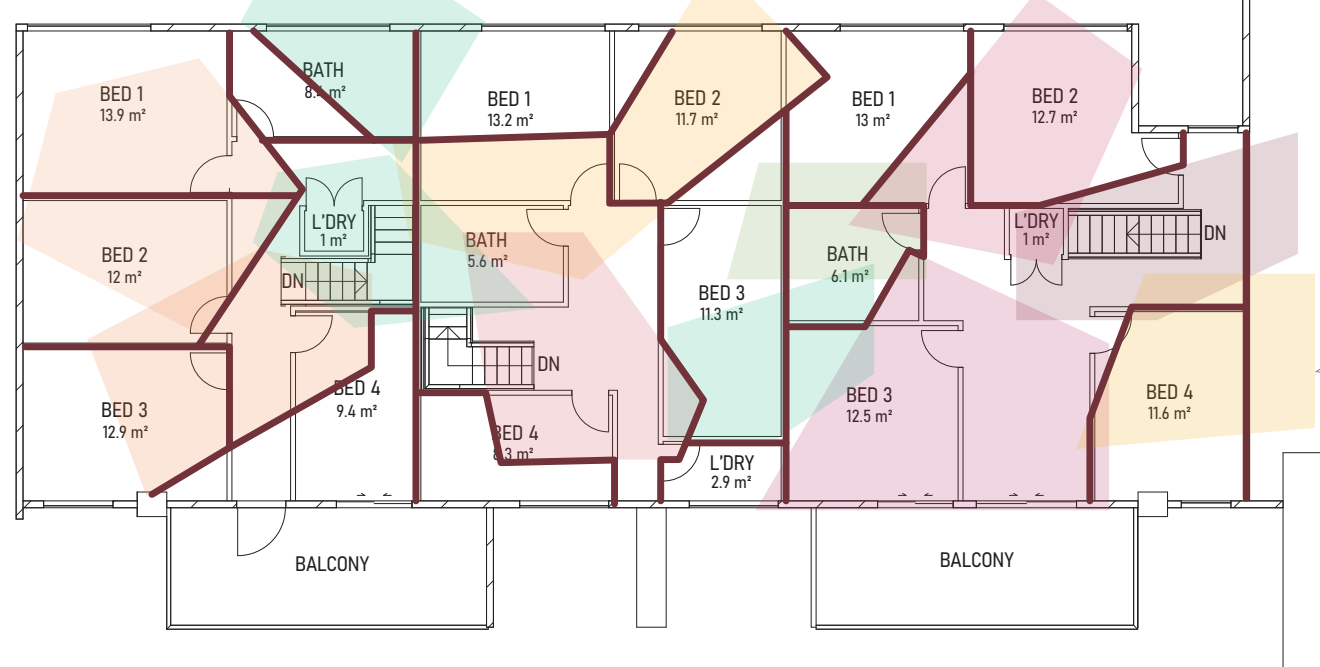

Floor Plan Iteration 2 - Level 2

1:200 


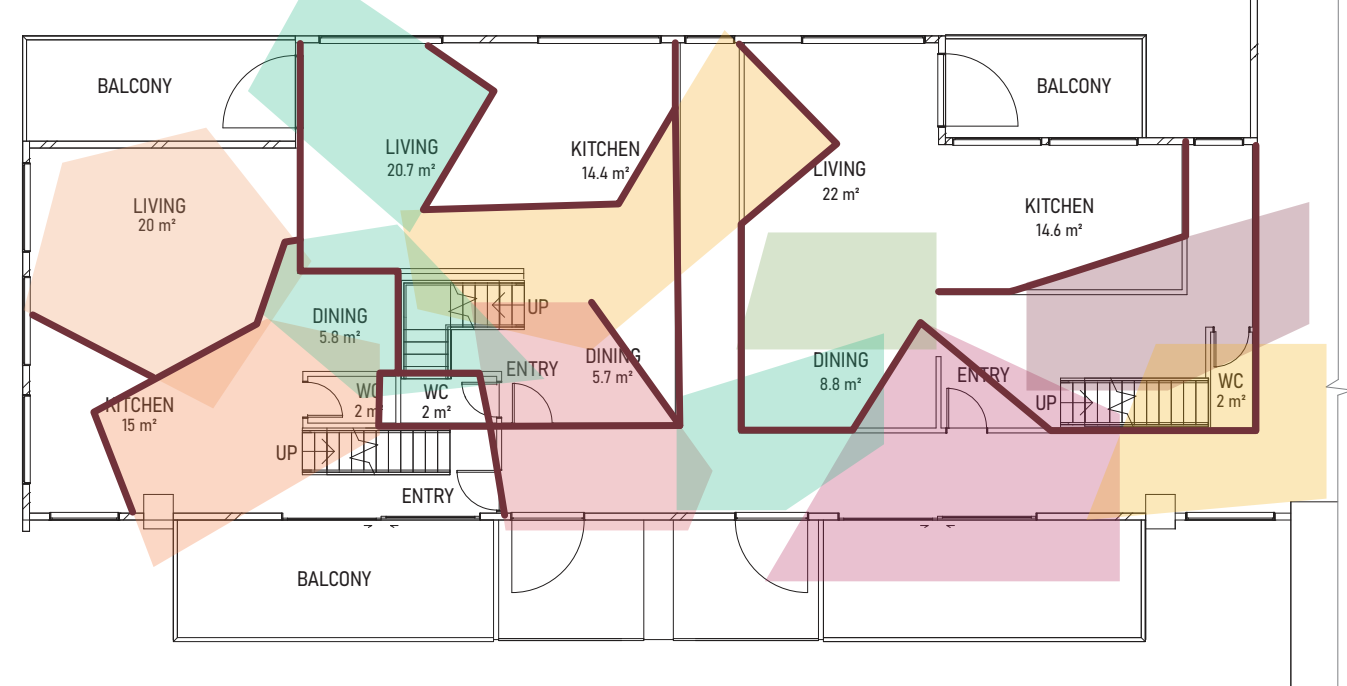

Floor Plan Iteration 3 - Level 1

$1: 200$

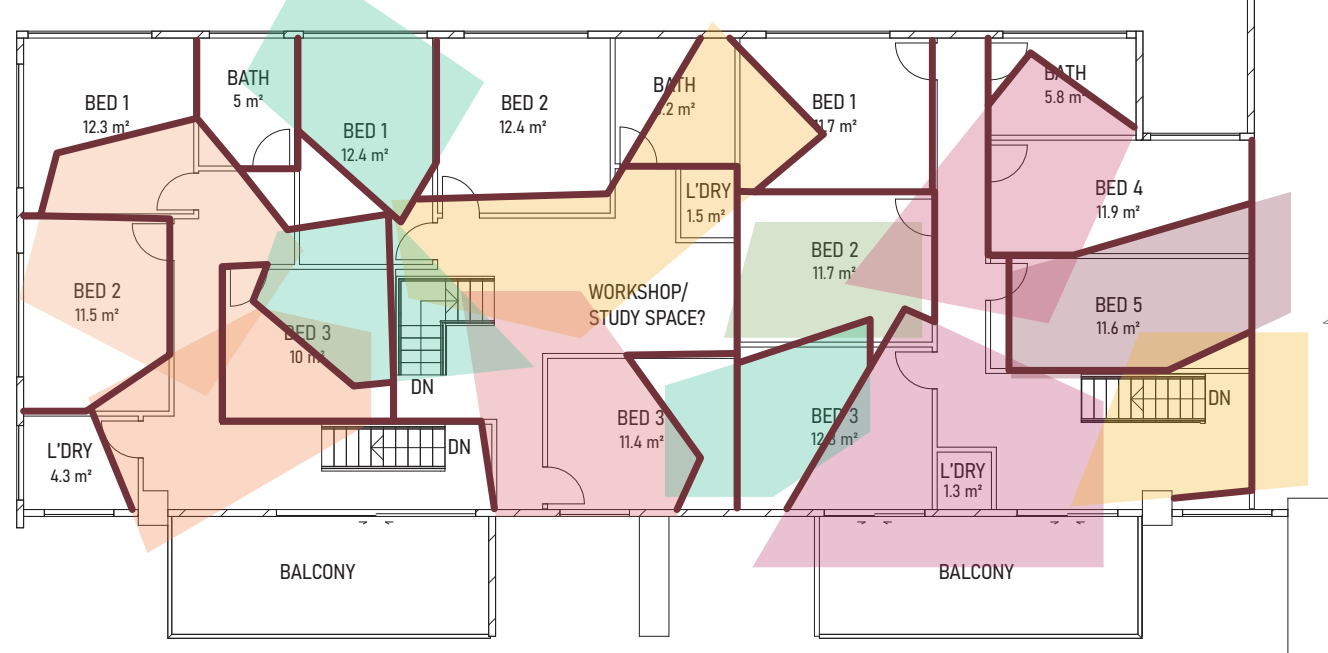

Floor Plan Iteration 3 - Level 2

1:200

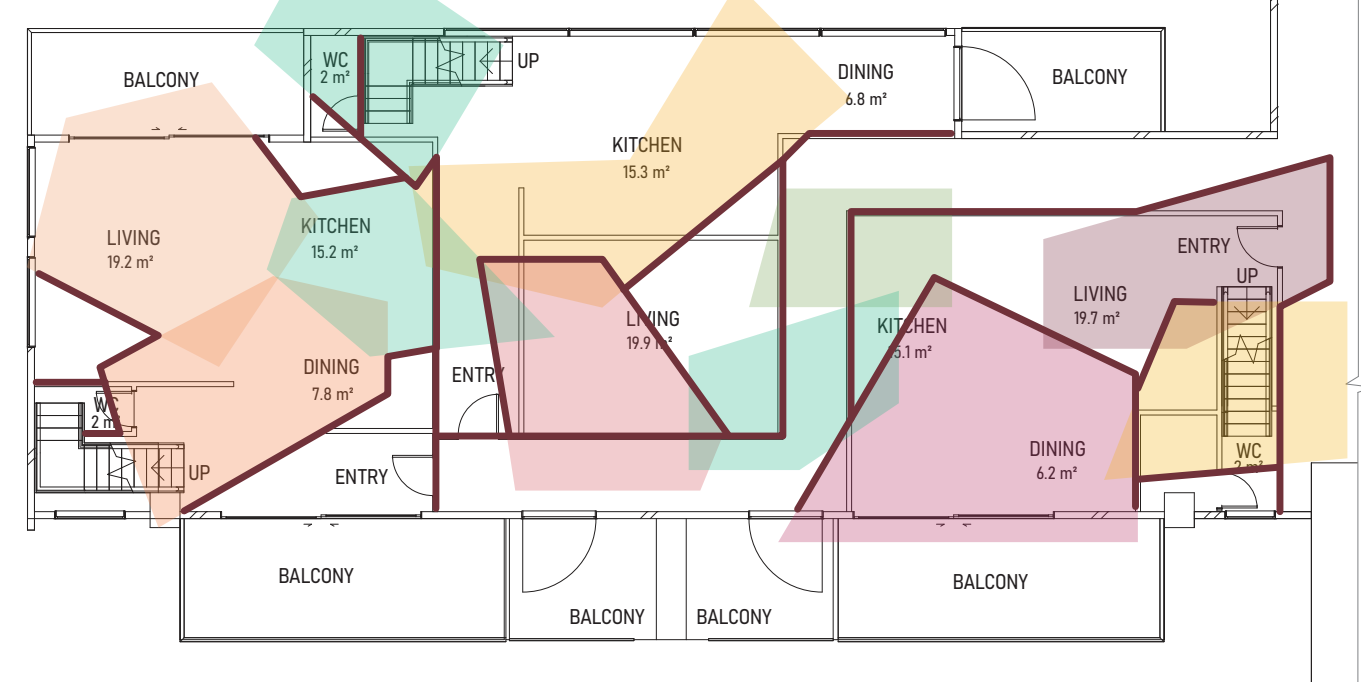

Floor Plan Iteration 4 - Level 1

$1: 200$

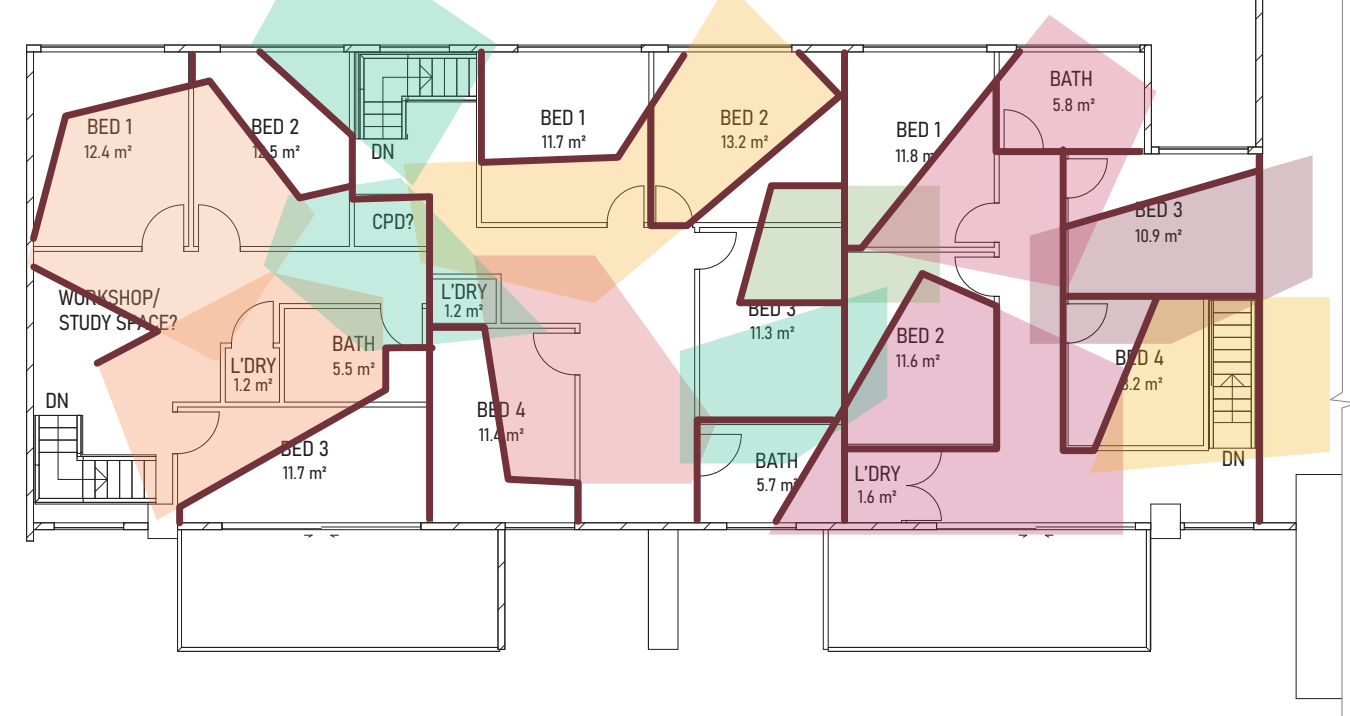

Floor Plan Iteration 4 - Level 2

1: 200 


\section{THE DISTURBANCE - PHASE 2}

New walls

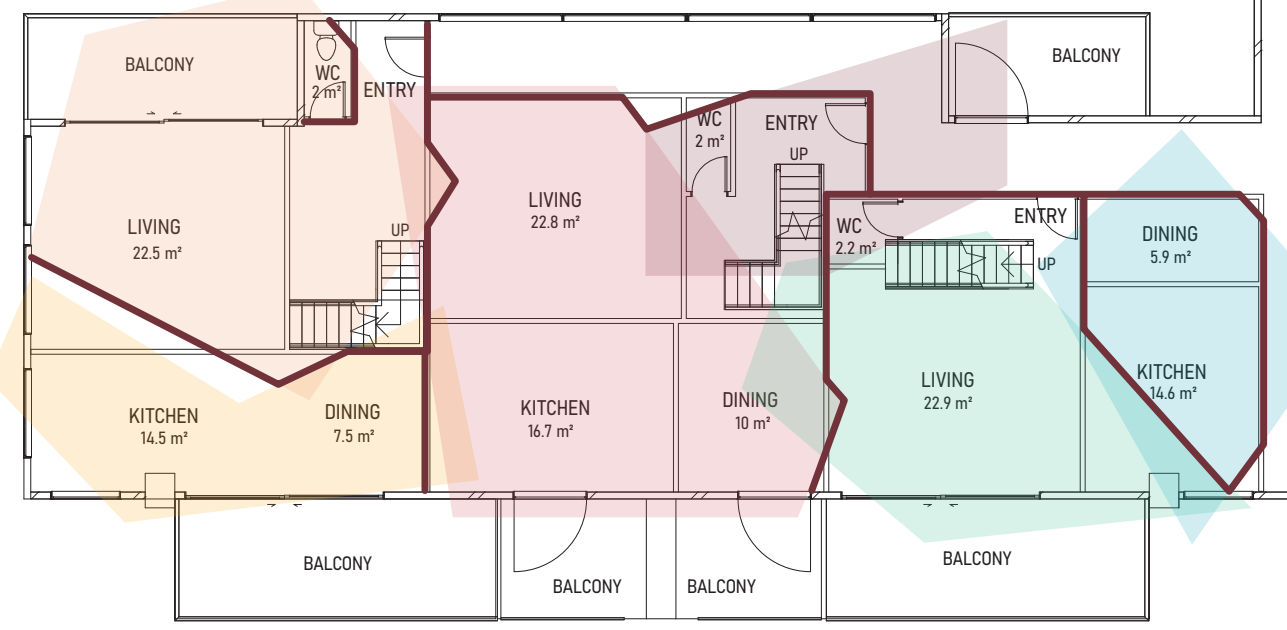

Floor Plan Iteration 1 - Level 1 1:200

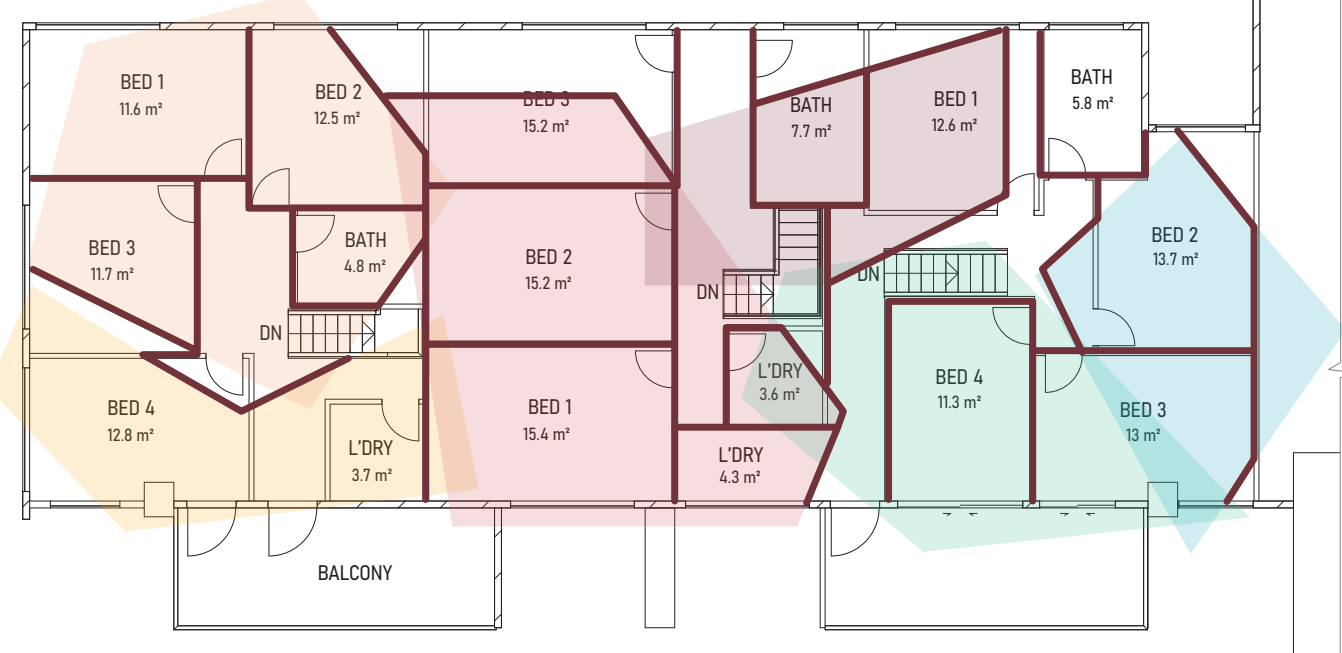

Floor Plan Iteration 1 - Level 2 1:200

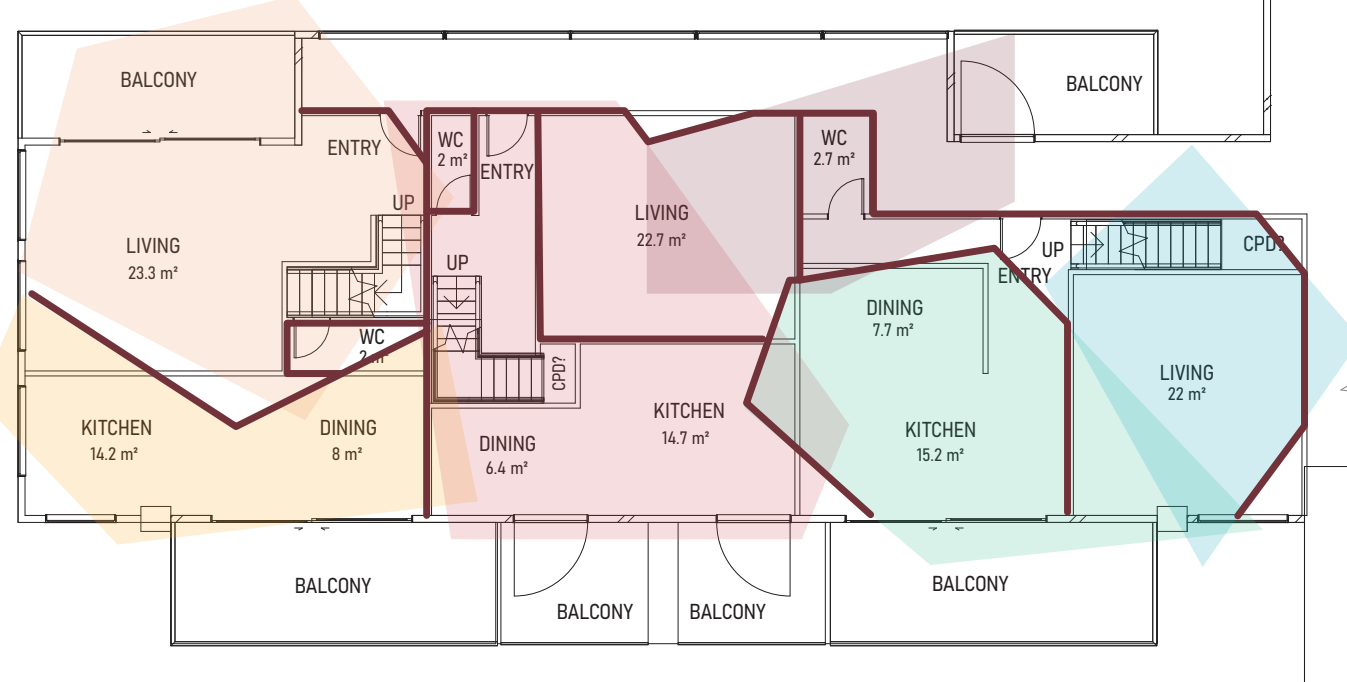

Floor Plan Iteration 2 - Level 1 $1: 200$

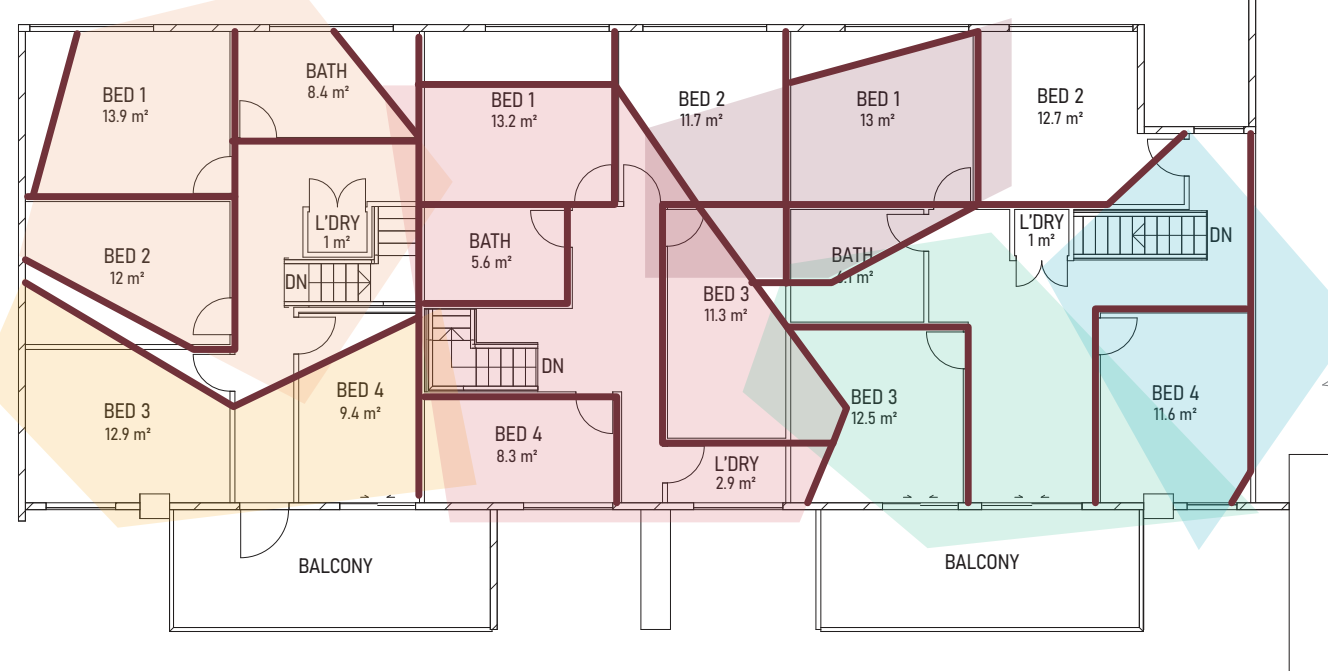

Floor Plan Iteration 2 - Level 2

1: 200 


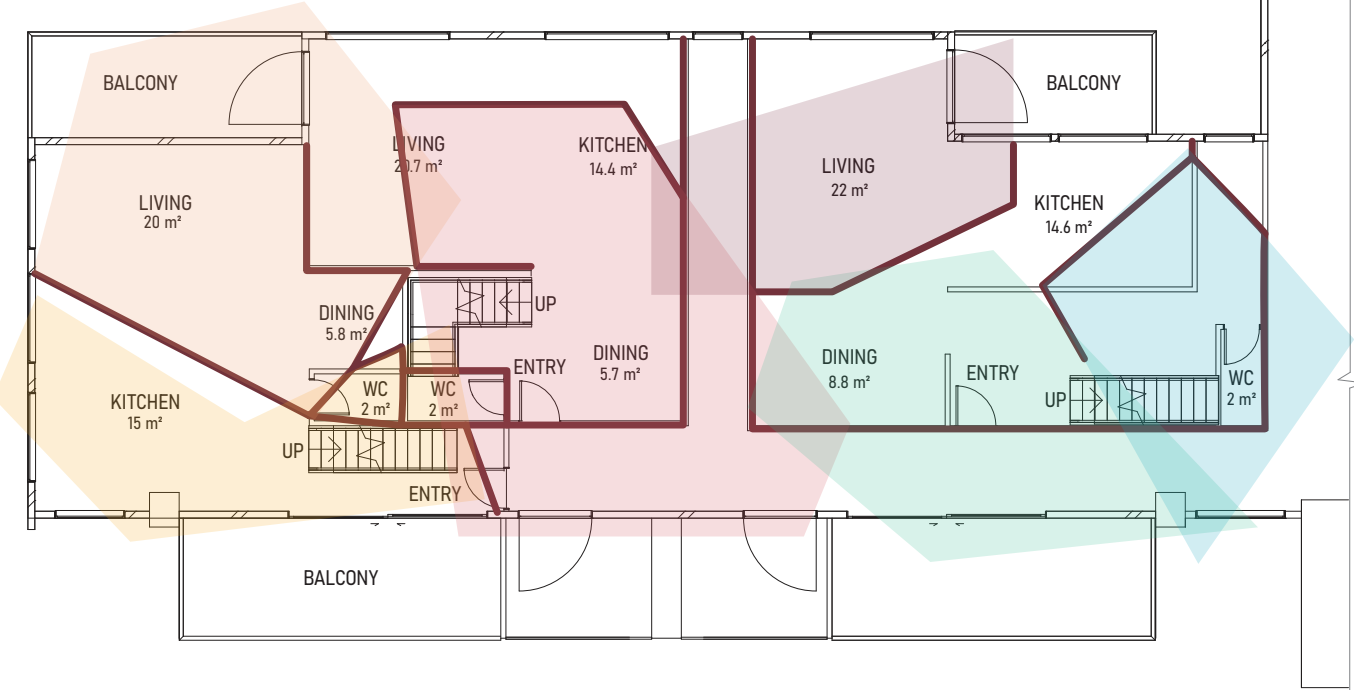

Floor Plan Iteration 3 - Level 1

1: 200

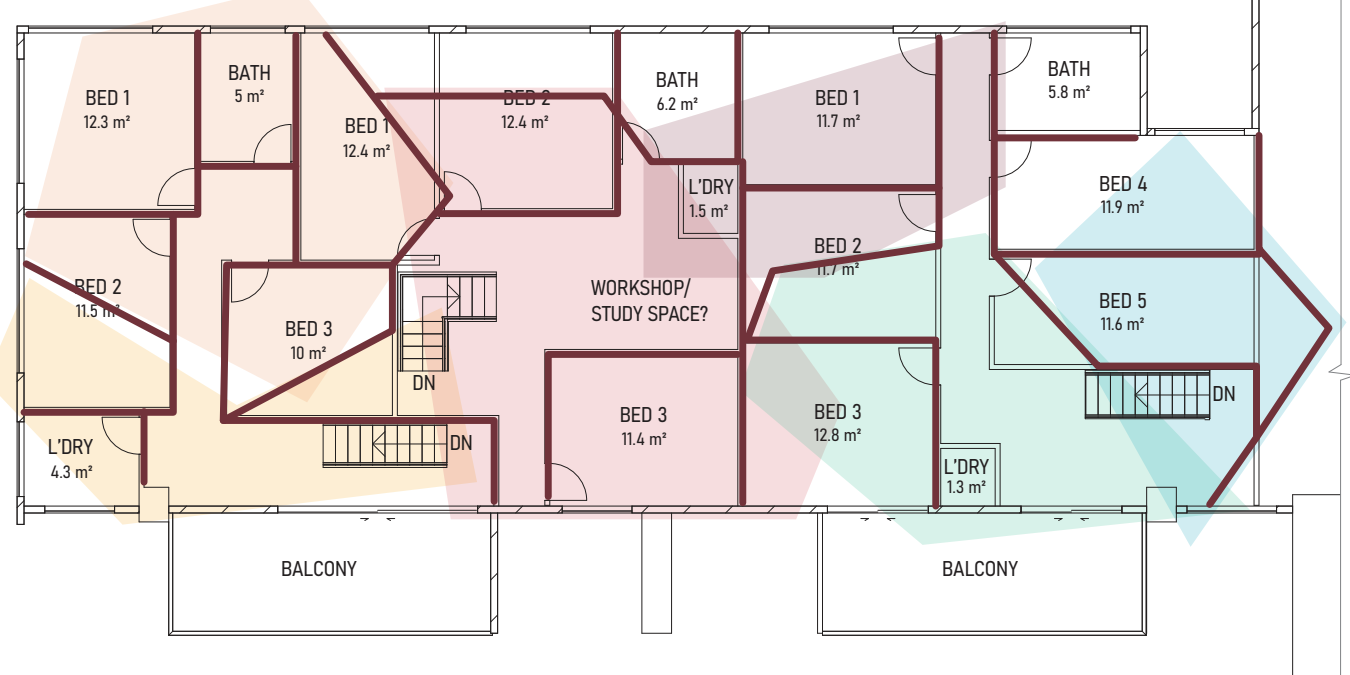

Floor Plan Iteration 3 - Level 2

1: 200

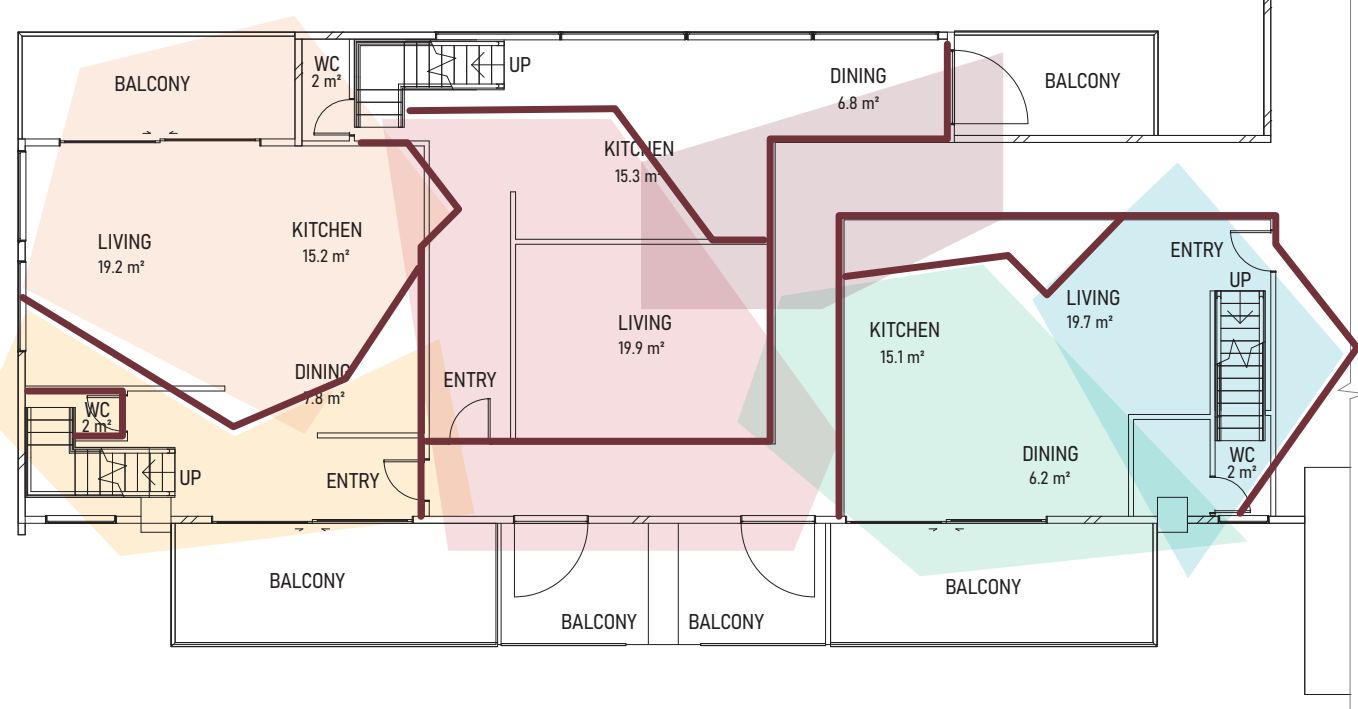

Floor Plan Iteration 4 - Level 1

1: 200

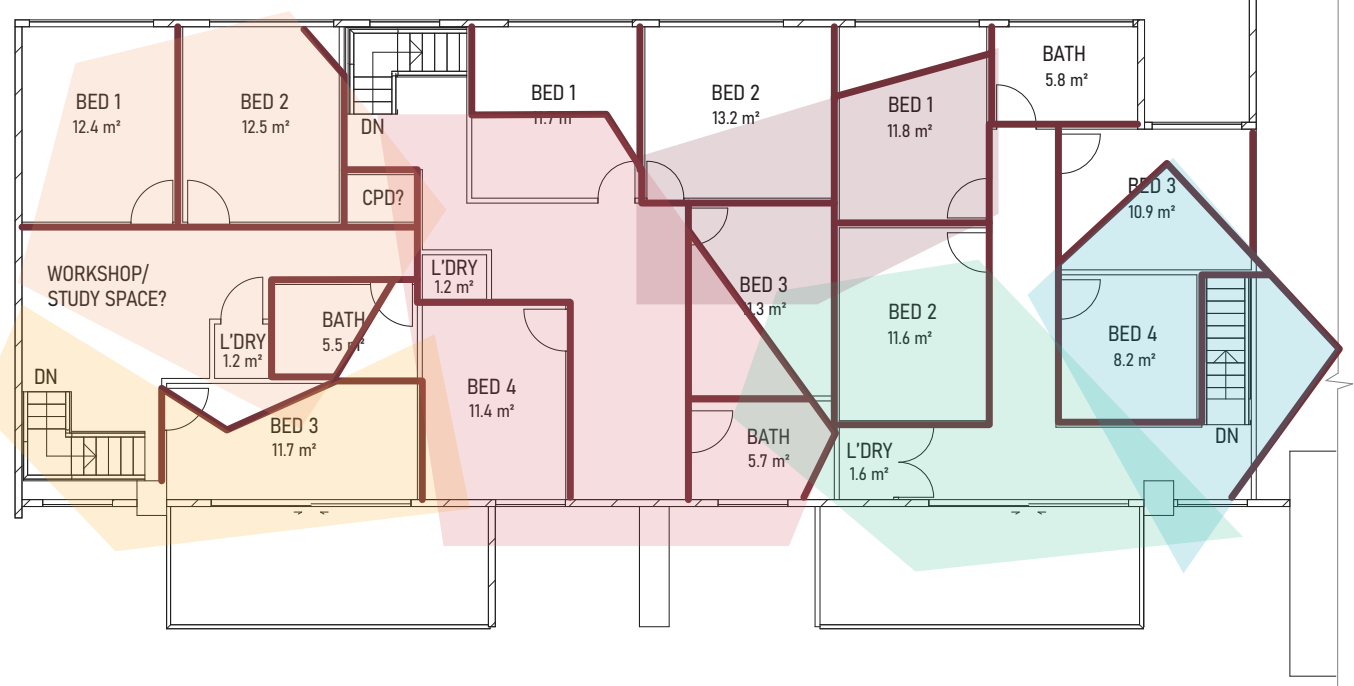

Floor Plan Iteration 4 - Level 2

1: 200 


\section{THE DISTURBANCE - PHASE 3}

New walls

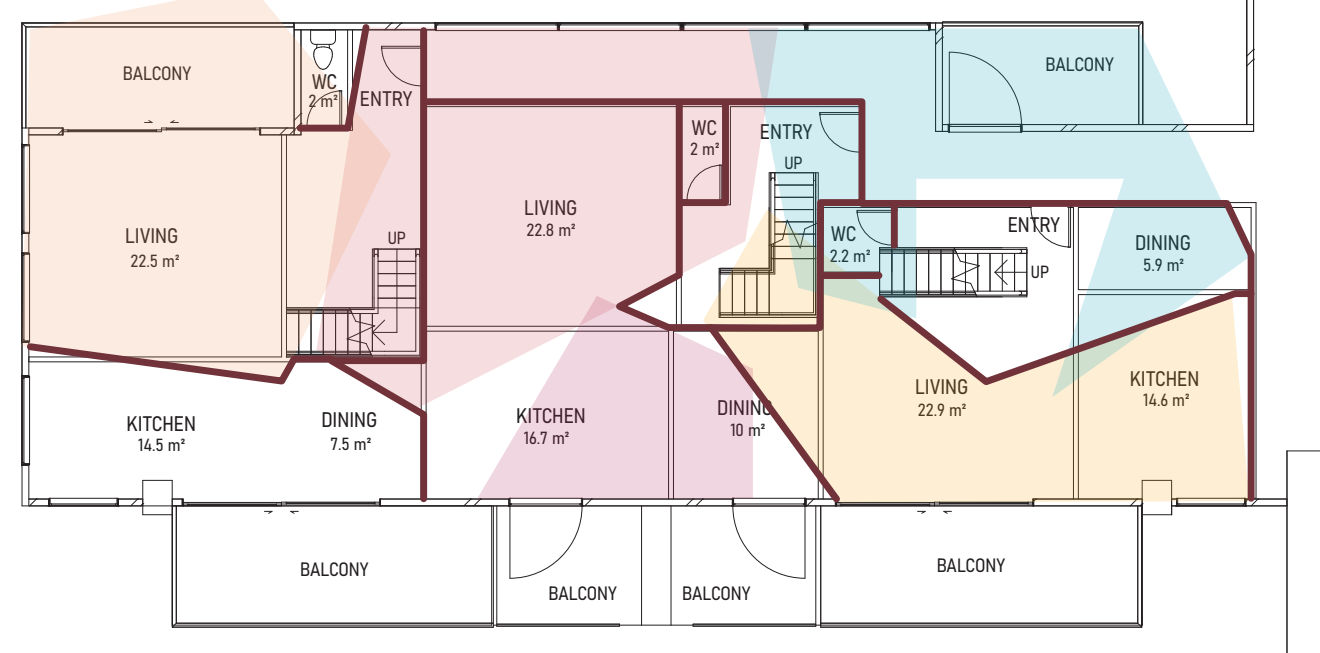

Floor Plan Iteration 1 - Level 1

$1: 200$

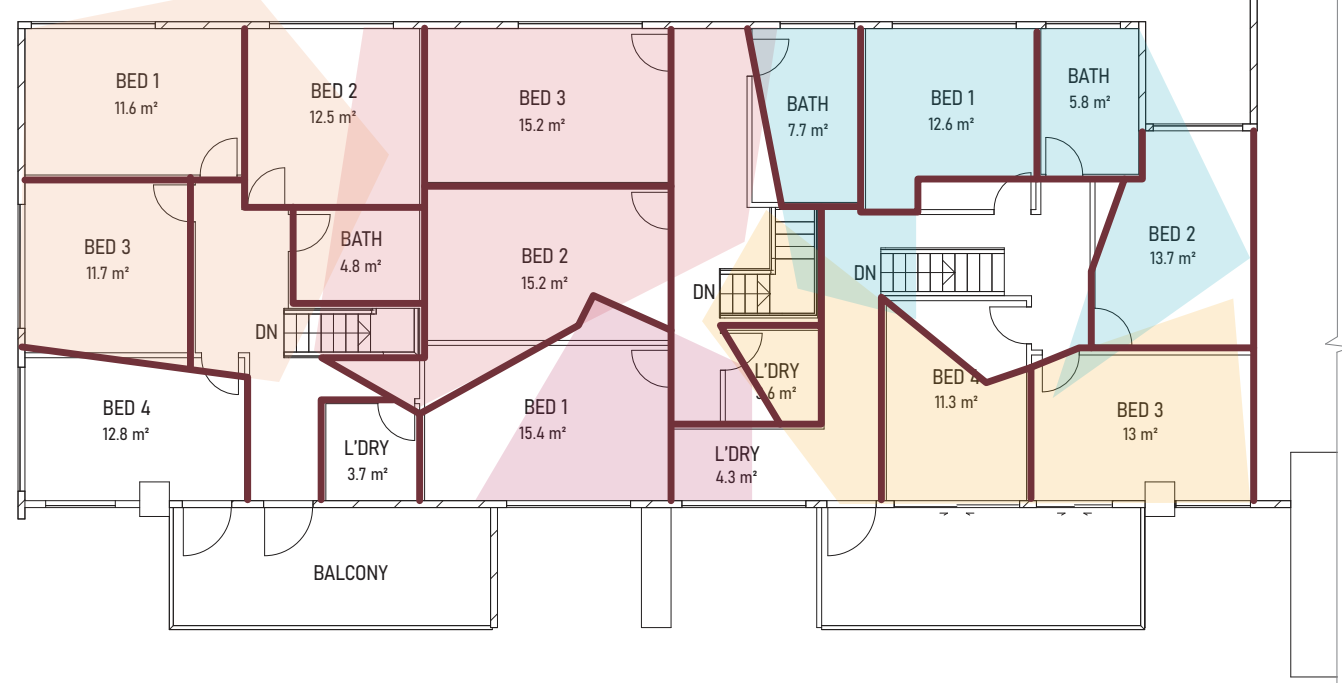

Floor Plan Iteration 1 - Level 2

1:200

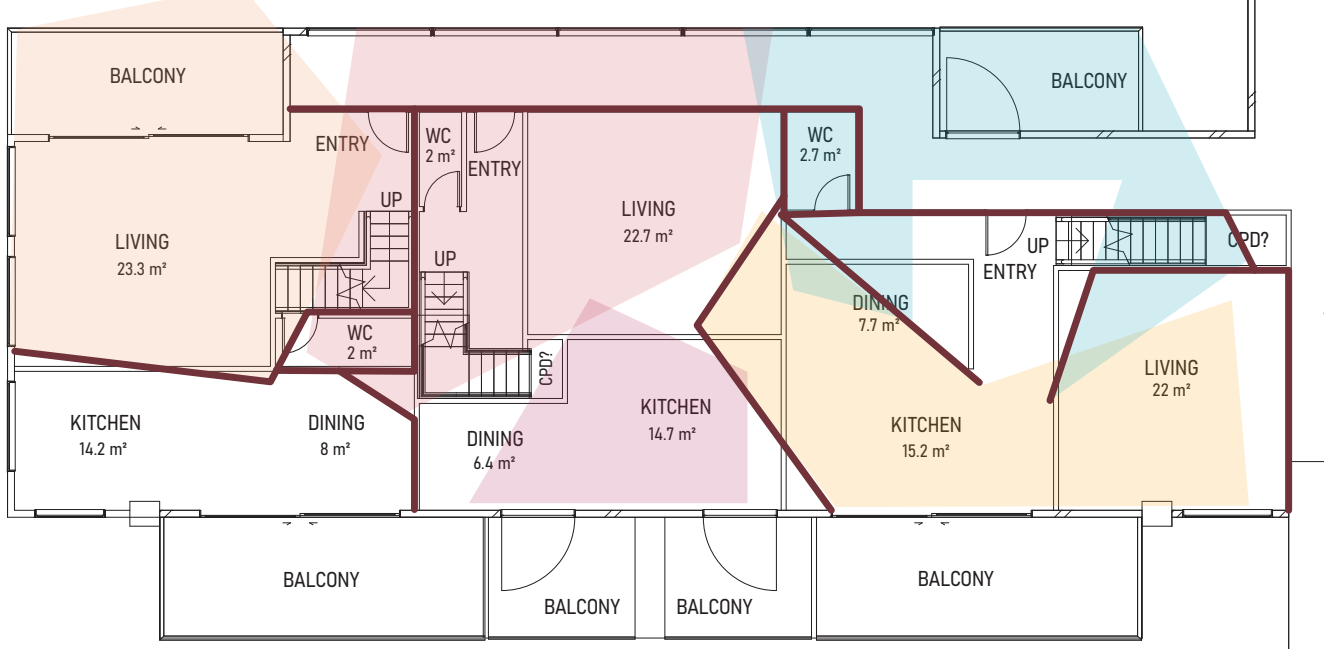

Floor Plan Iteration 2 - Level 1

$1: 200$

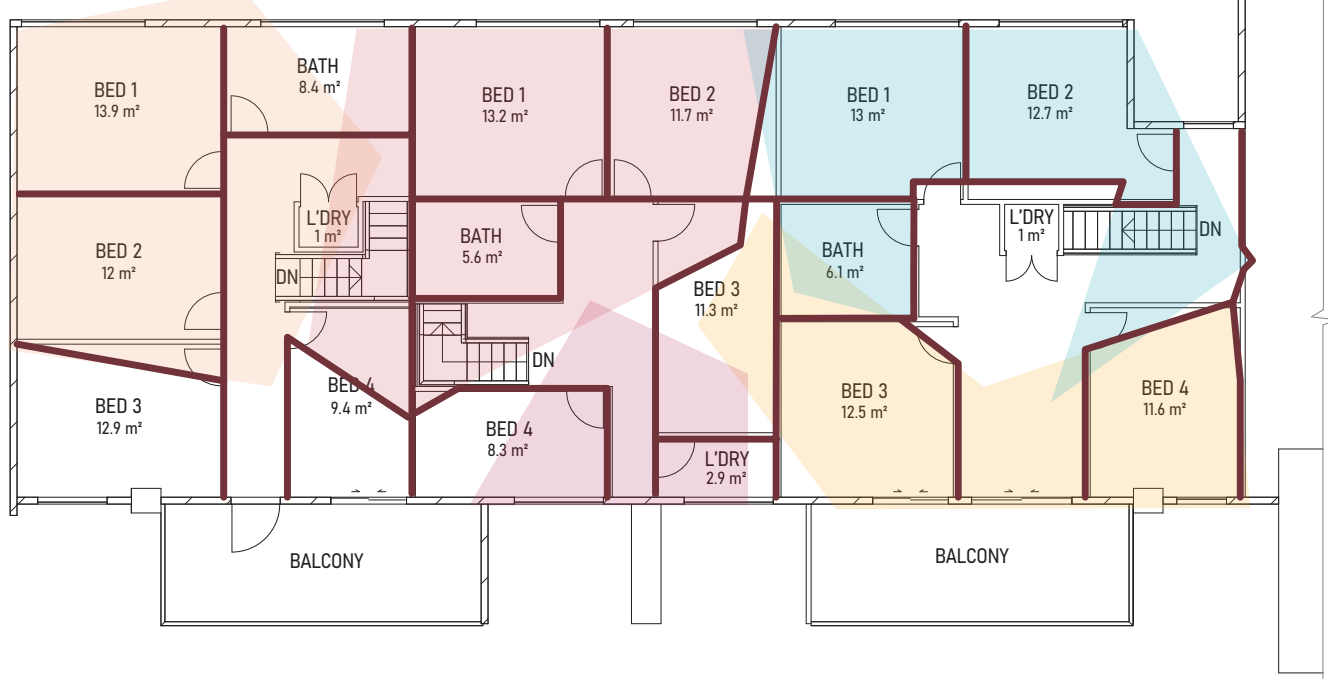

Floor Plan Iteration 2 - Level 2

1:200 


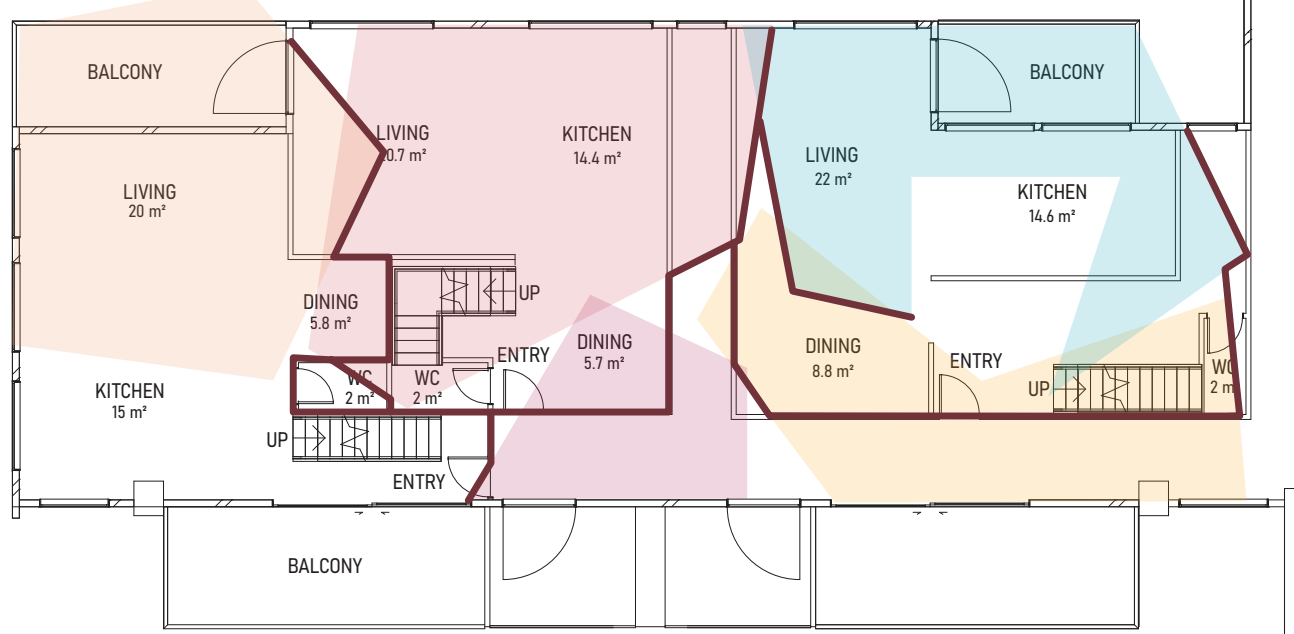

Floor Plan Iteration 3 - Level 1

$1: 200$

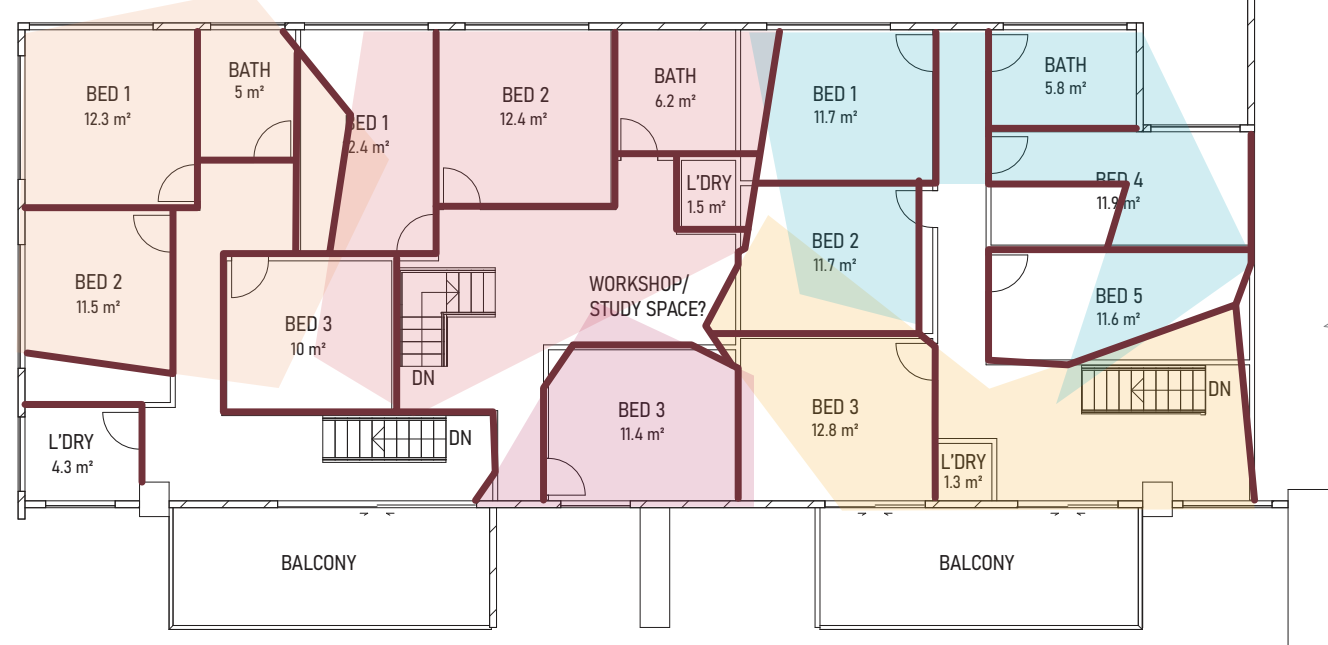

Floor Plan Iteration 3 - Level 2

1:200

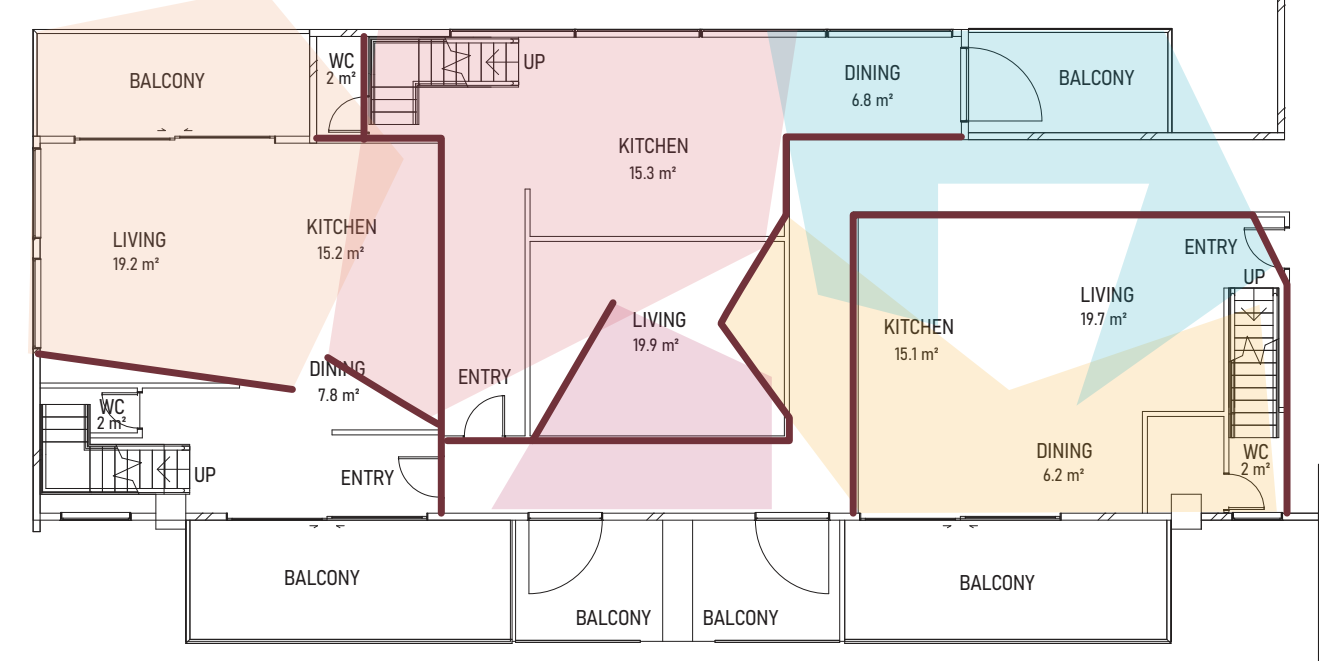

Floor Plan Iteration 4 - Level 1

1:200

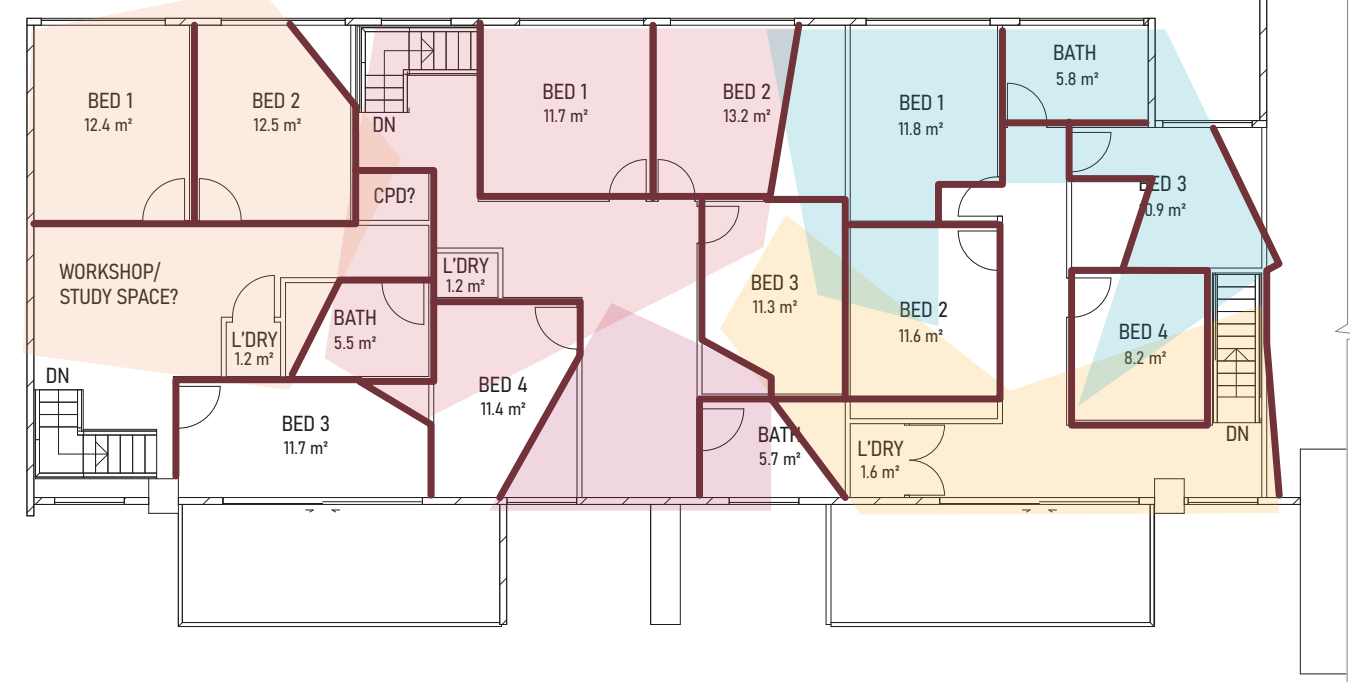

Floor Plan Iteration 4 - Level 2

1: 200 


\section{THE DISTURBANCE - PHASE 4}

_ New walls

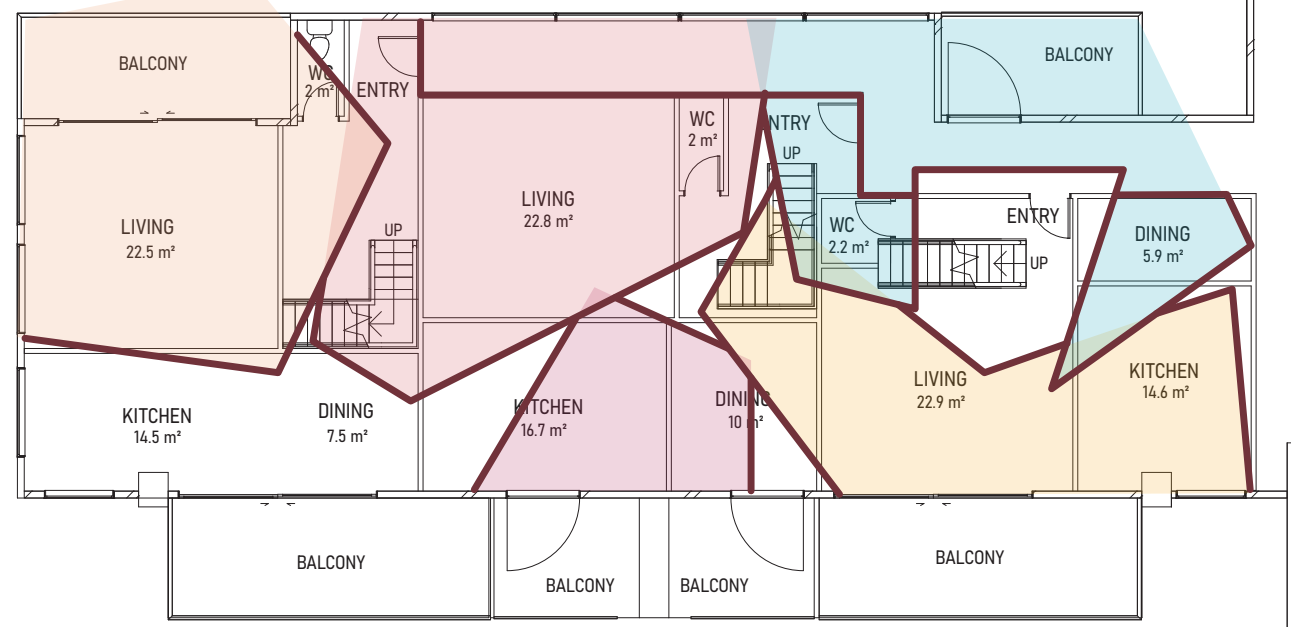

Floor Plan Iteration 1 - Level 1

$1: 200$

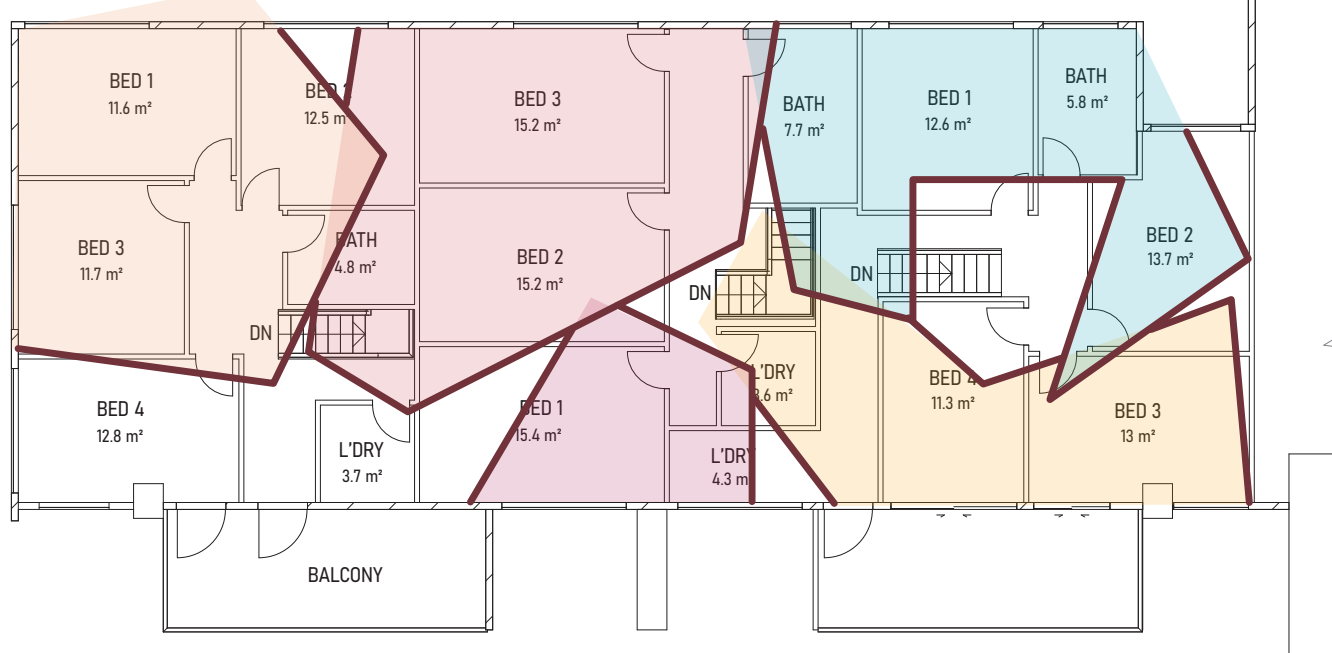

Floor Plan Iteration 1 - Level 2

1:200

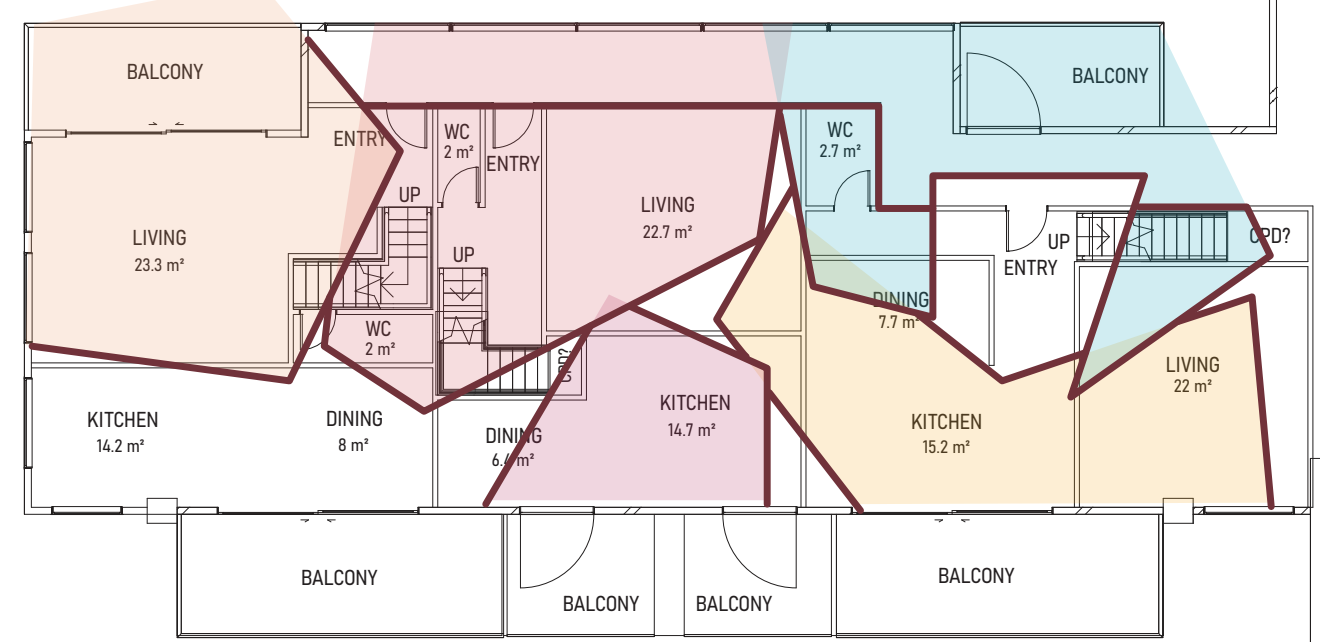

Floor Plan Iteration 2 - Level 1

1: 200

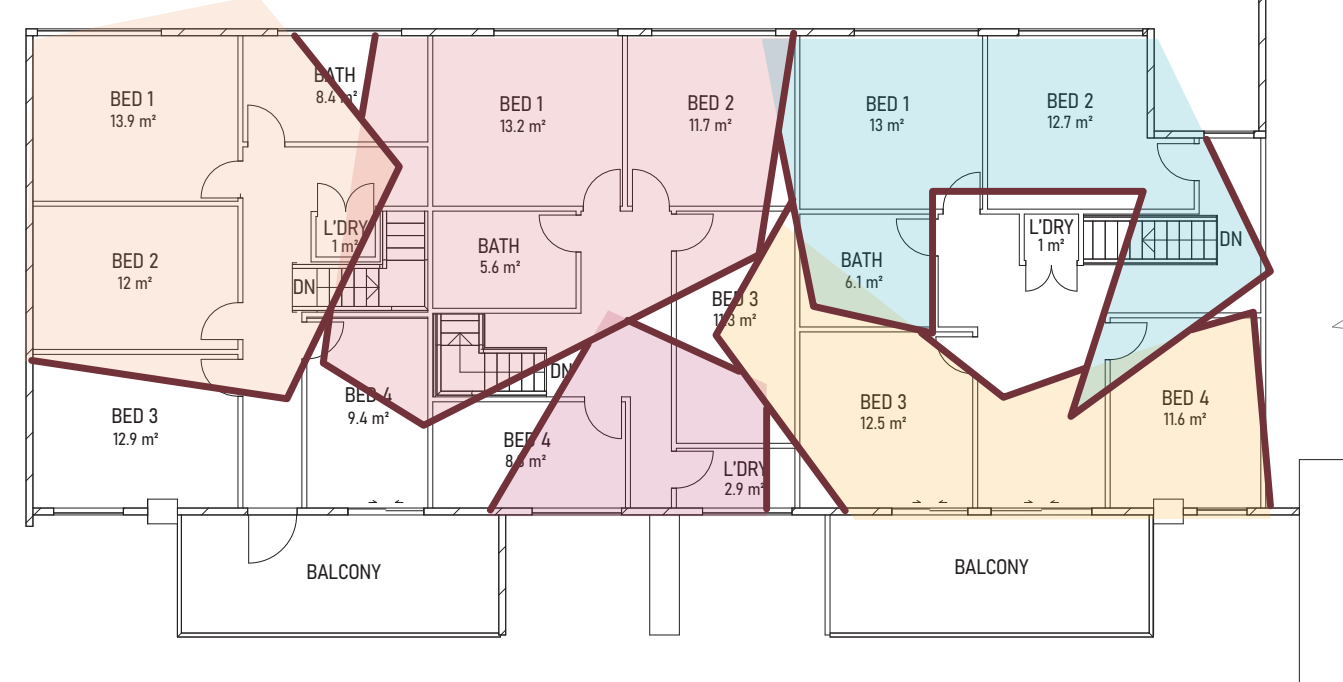

Floor Plan Iteration 2 - Level 2

$1: 200$ 


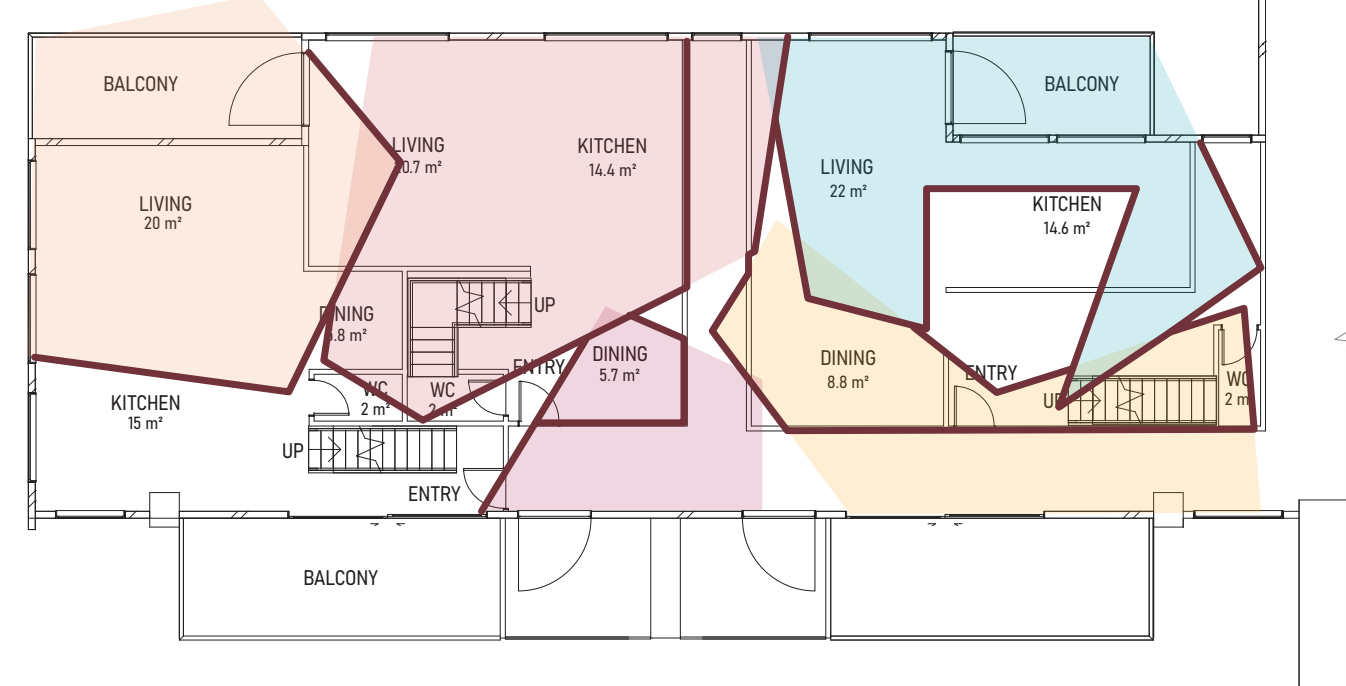

Floor Plan Iteration 3 - Level 1

1:200

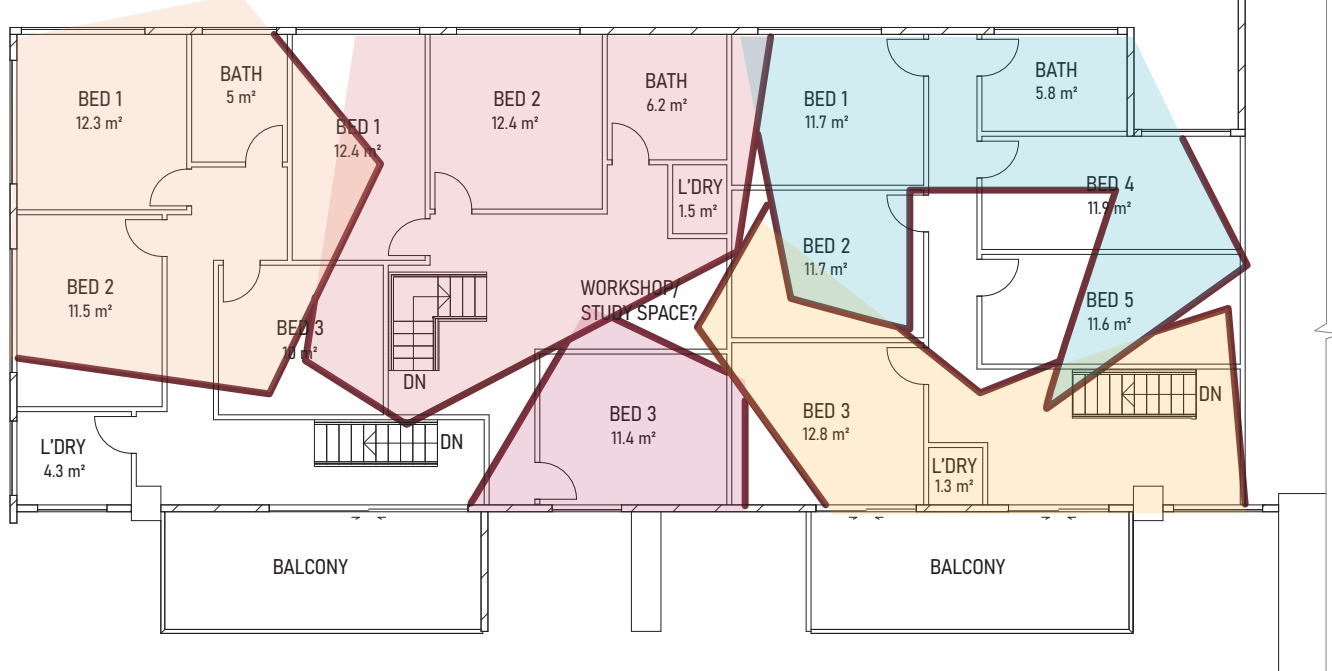

Floor Plan Iteration 3 - Level 2

1: 200

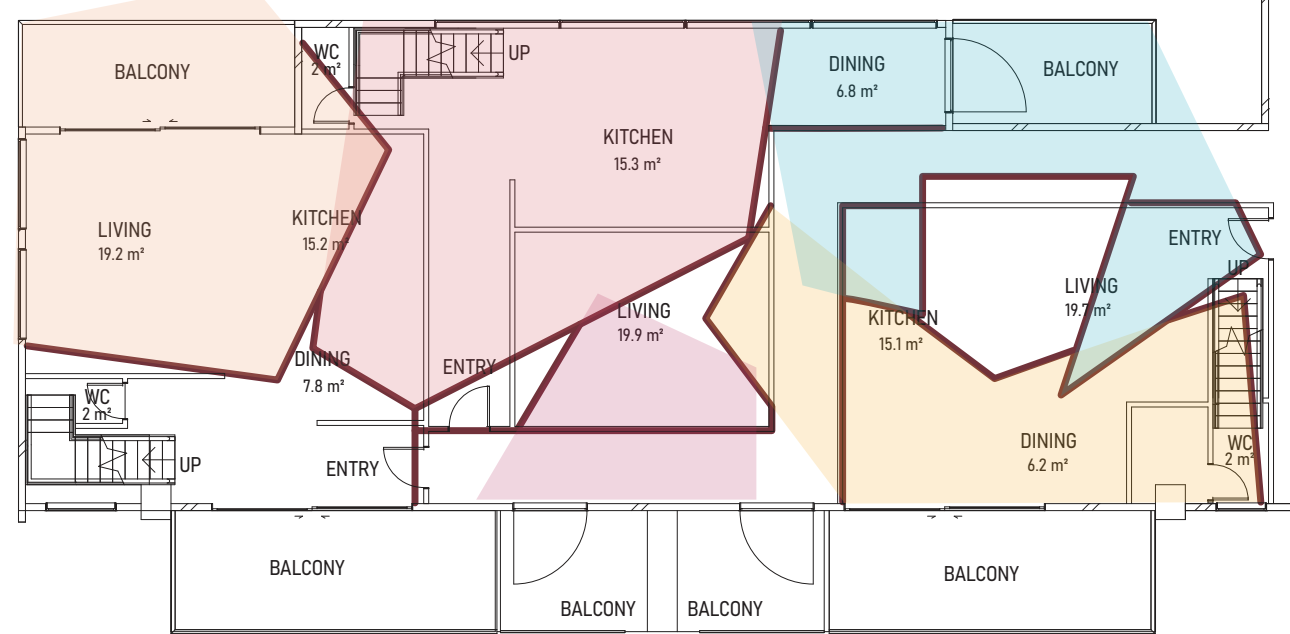

Floor Plan Iteration 4 - Level 1

1: 200

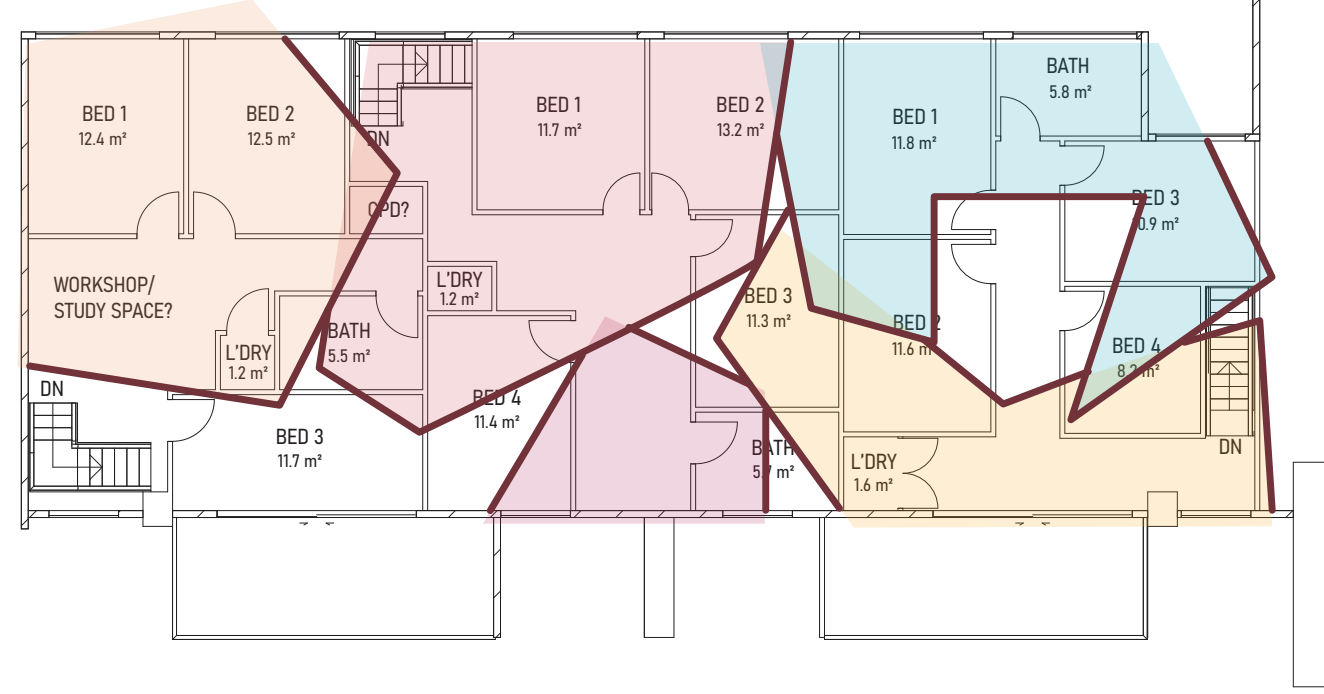

Floor Plan Iteration 4 - Level 2

1:200 


\section{PHASE 4}

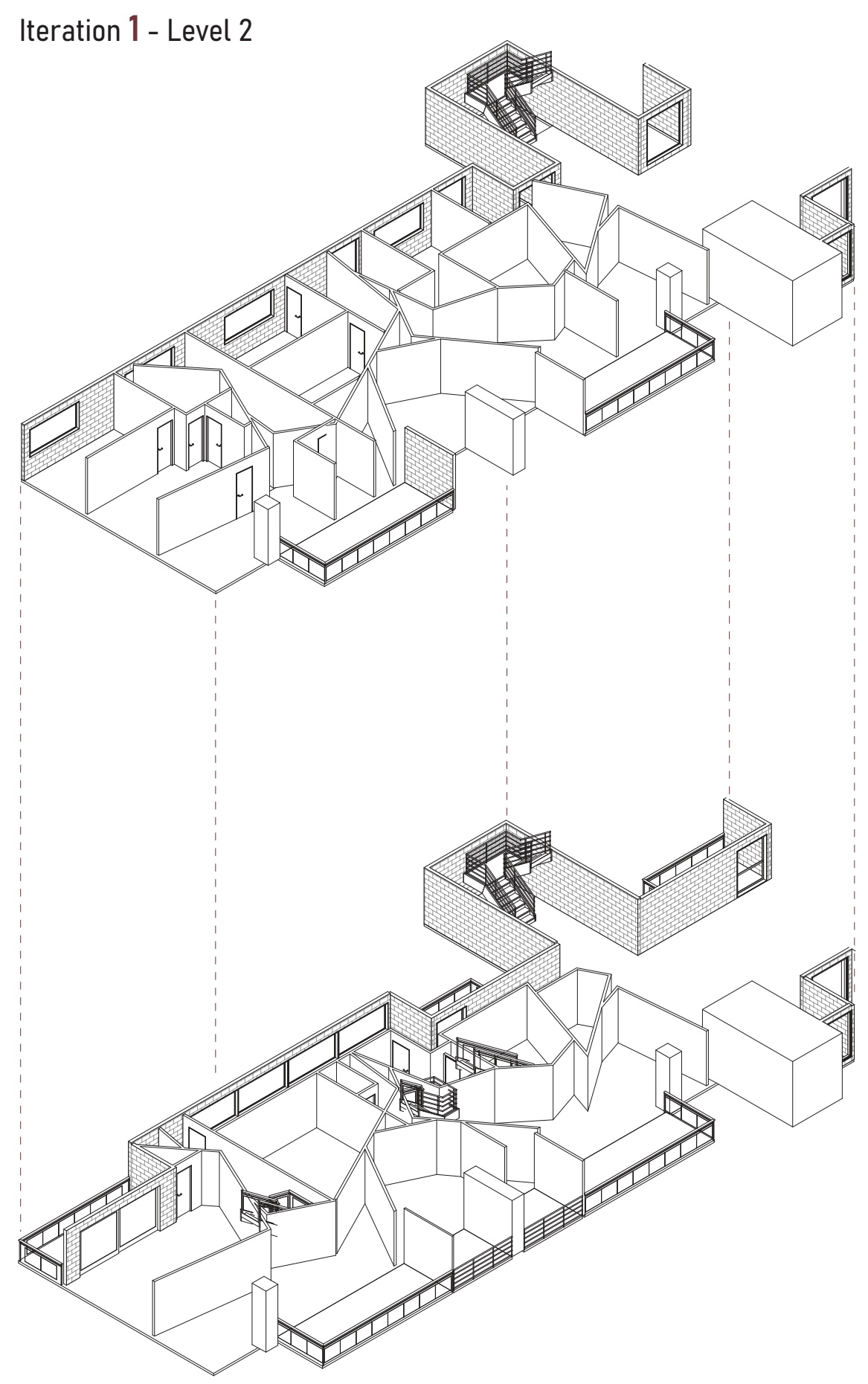

Iteration 1 - Level 1

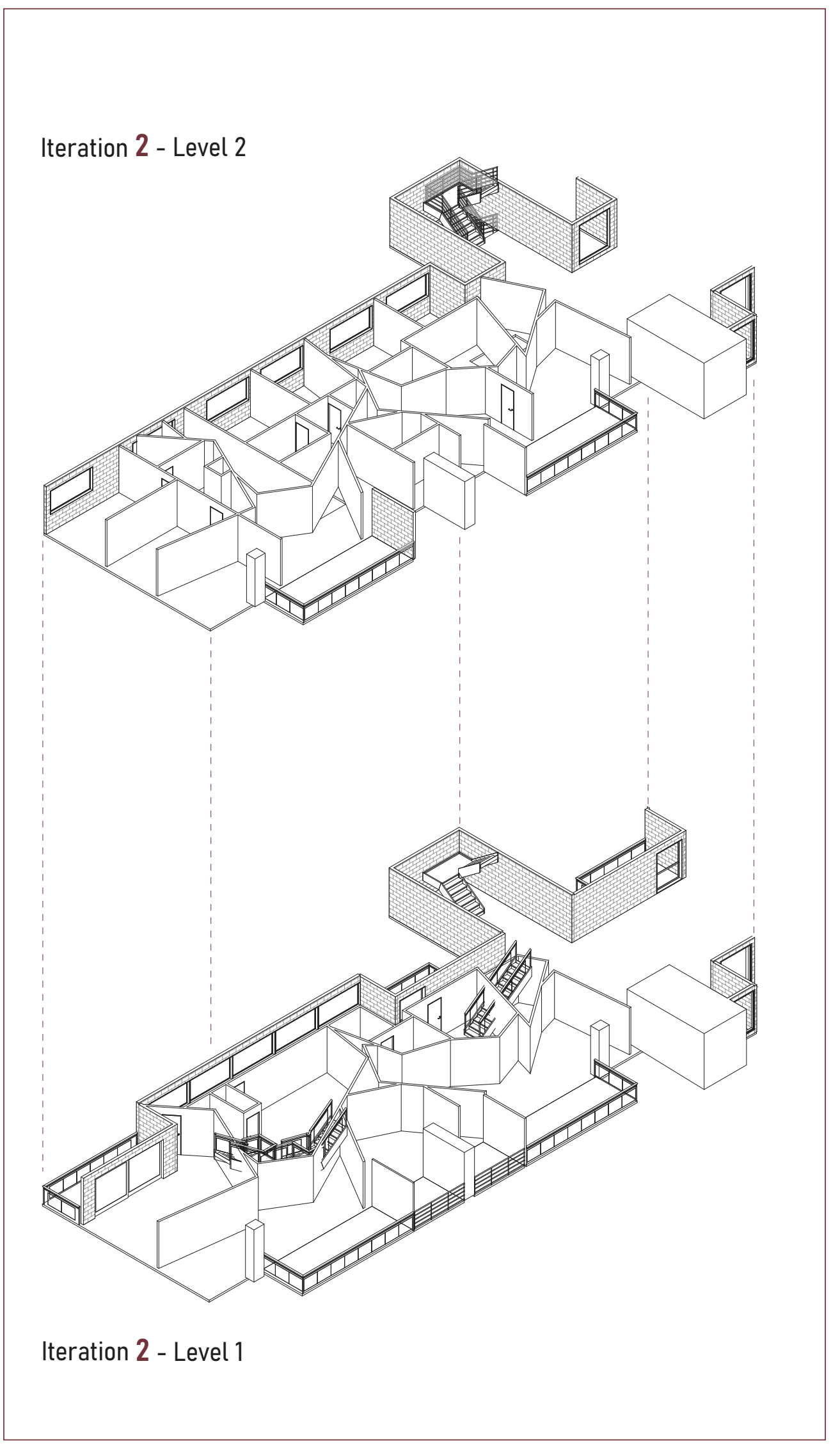




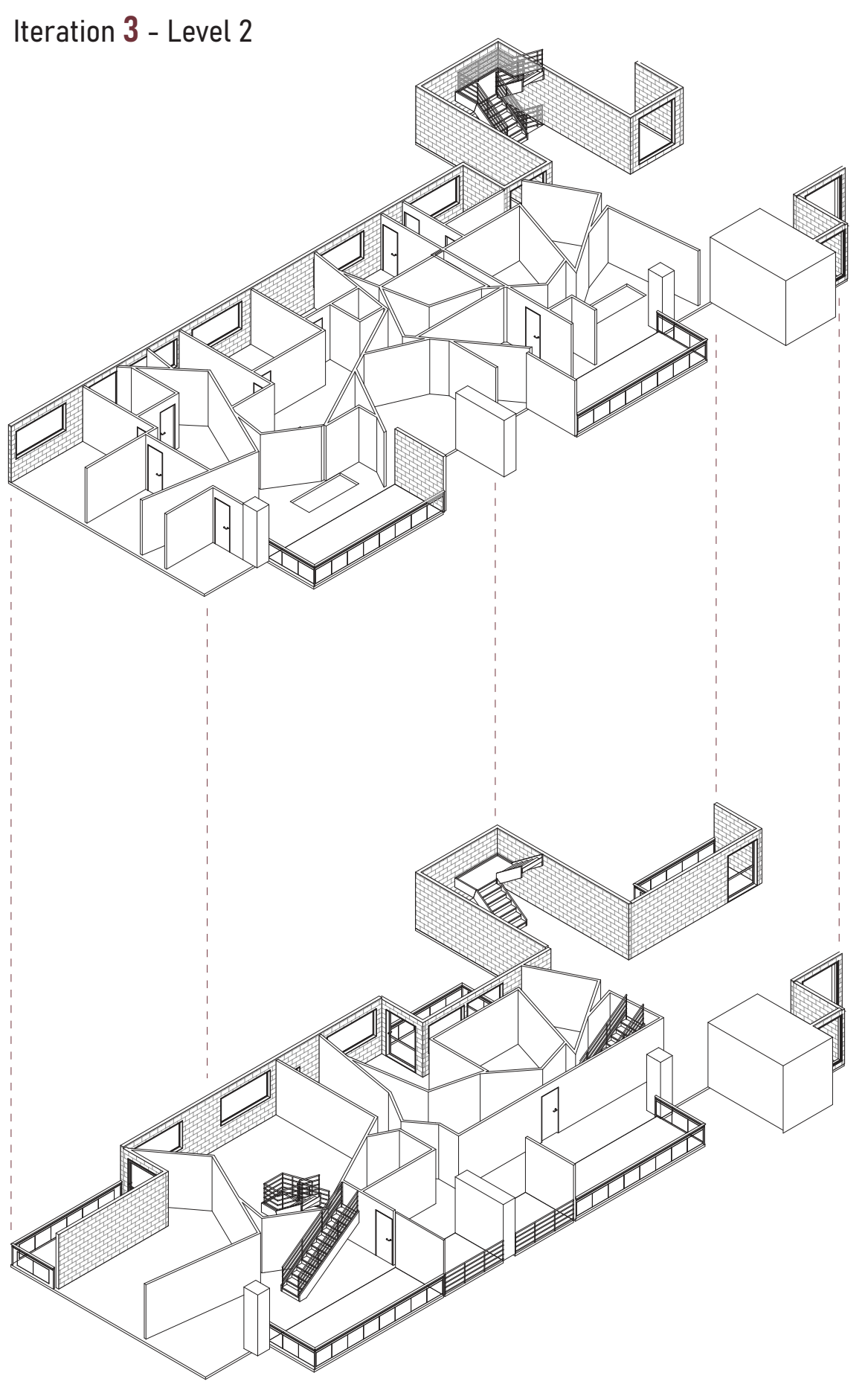

Iteration 3 - Level 1

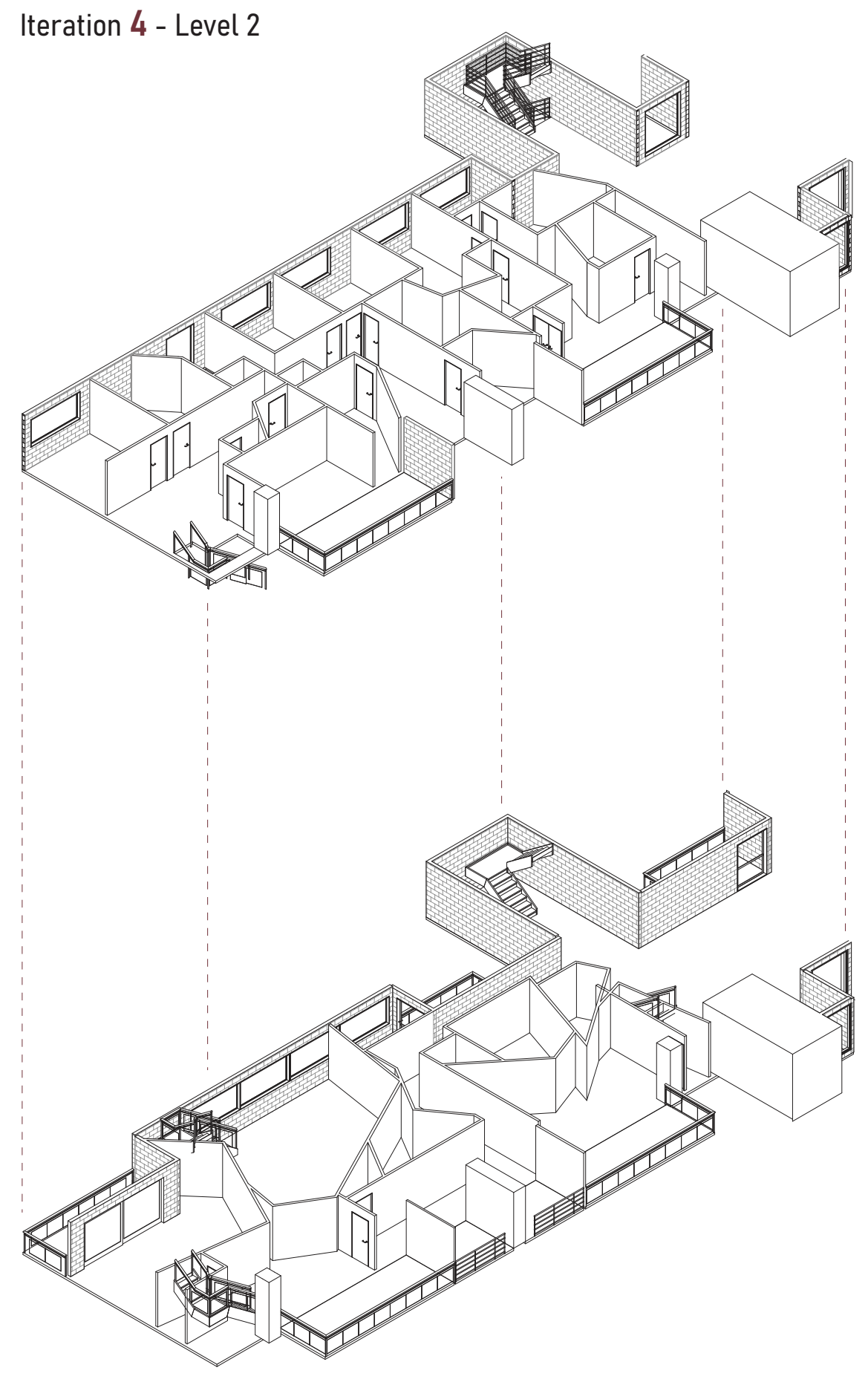

Iteration 4 - Level 1 


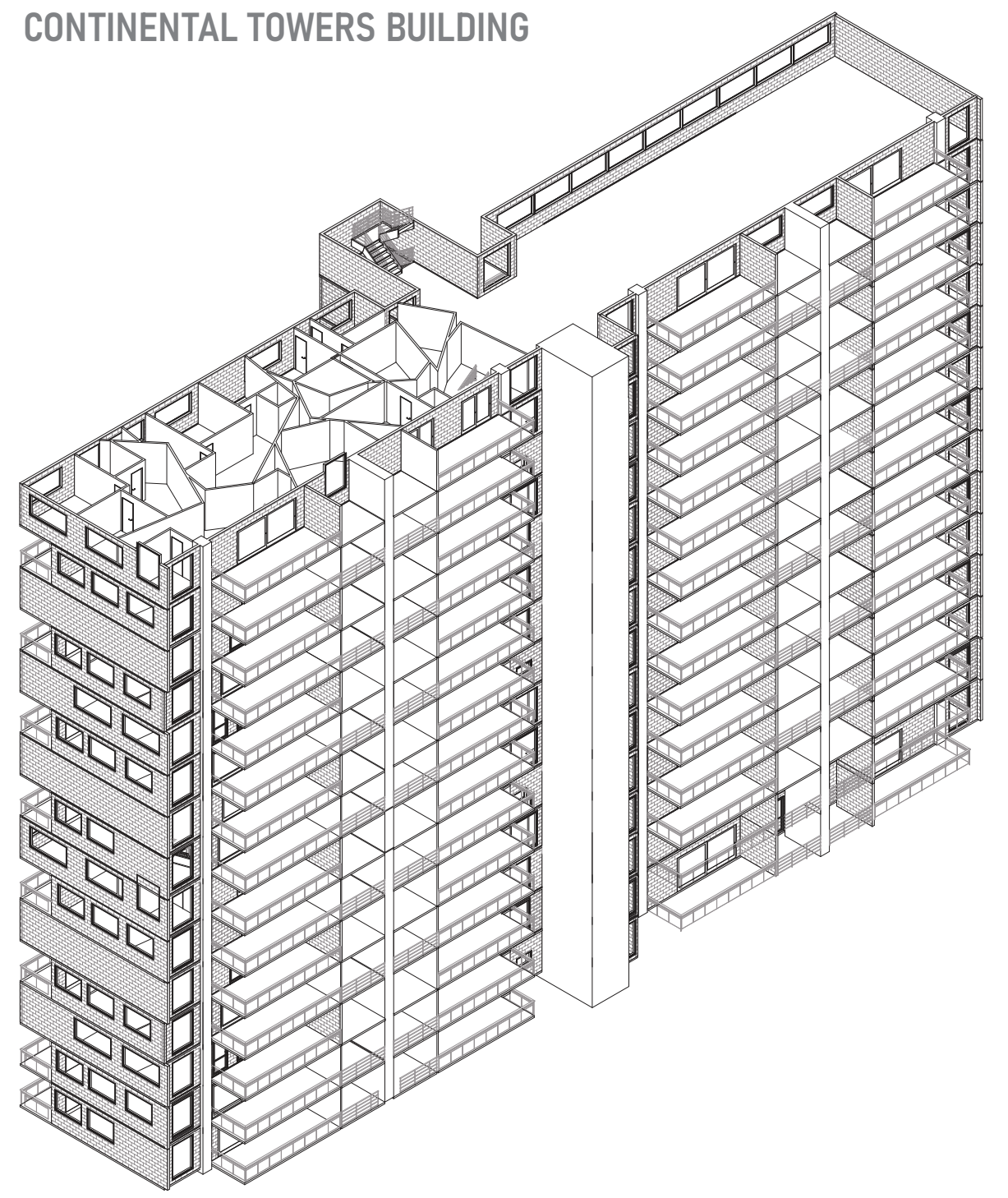

15 FLOORS

ALL DOUBLE LEVEL APARTMENTS

GROUND FLOOR TO BE COMMUNITY CENTER

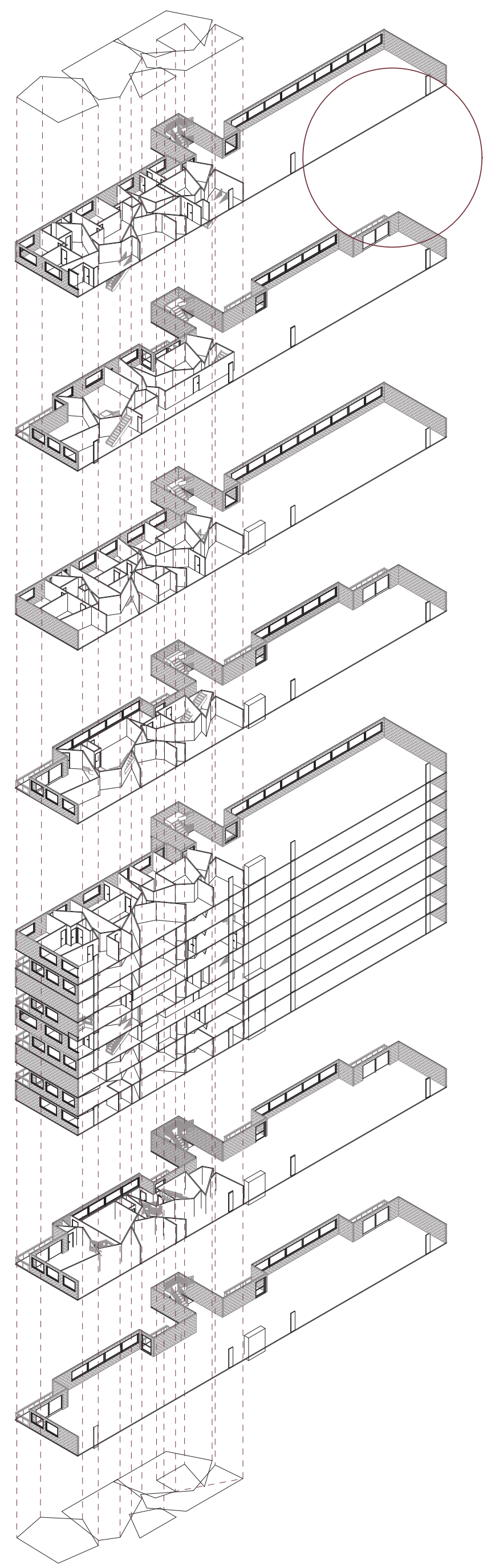




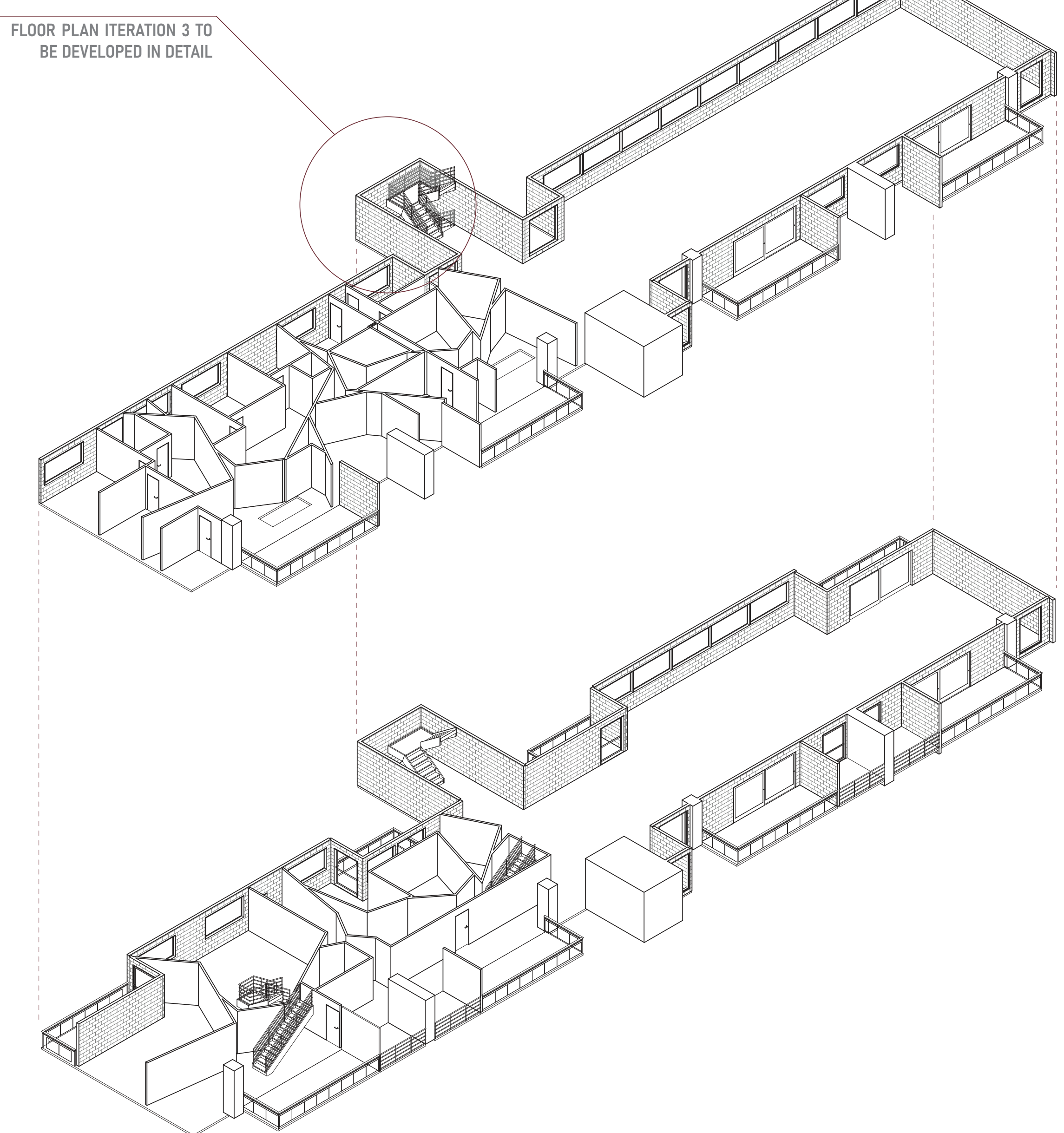




\section{Conclusions + Reflections}

\section{Conclusiones + reflexiones}

This programme was inspired by not only the apparent need this community has for better housing and living but also their clear want to move away from their current situation and have better lives.

The design for this housing needed to not only give them better living conditions but it also needed to become an expression of them and be inspired by their art. This becomes linked to the ideas of place identity explored earlier whilst being linked to the research on adaptive re-use, high density housing and the way in which families are structured in Colombia.

The key aims for this design were to create housing which felt like it truly belongs to La Comuna 13. Using the re-coding of their art to create shapes which influenced the layout of the walls within their homes. To create a space which not only continues to enable their artistic expression but also encourages it through the continuing use of the wall as a canvas. Being at a conceptual stage, the design is reaching these aims thus far. The interior architecture is starting to be influenced by the community's art and becoming expression of both art and community from an interior architecture practice.
This is the concept which was chosen to fully develop as the final design for this design led-research. In the development stage it will be important to further test the changing orientation of walls and how this will affect the interior. It will also be significant to delve into the design of details such as door handles and balustrades as well as the design of custom furniture. All of these details will use similar processes of utilising the shapes extracted from the art and applying these into the design. And lastly, seeing as the community aspect with La Comuna 13 is so important, it will be crucial to design spaces which allow for community gatherings. A community centre which includes space for dance but also workshops and teaching will be created within the first floor of the building. And some smaller communal spaces will also be designed on every second floor of the building which will allow for the creation of smaller communities amongst neighbours within the larger community of the building. 


\section{"La juventud de La Comuna 13 quiere otra cosa. La gran ma- joria quiere cambia su realidad y futuro." - Kbala}

"The youth of La Comuna 13 want something else. The great majority want to change their reality and future." 


\section{CHAPTER 6: THE DEVELOPMENT}

Capítulo 6: El desarrollo 
Fin<smiles>C=PC=PC</smiles> 
1. HOME

CASA 


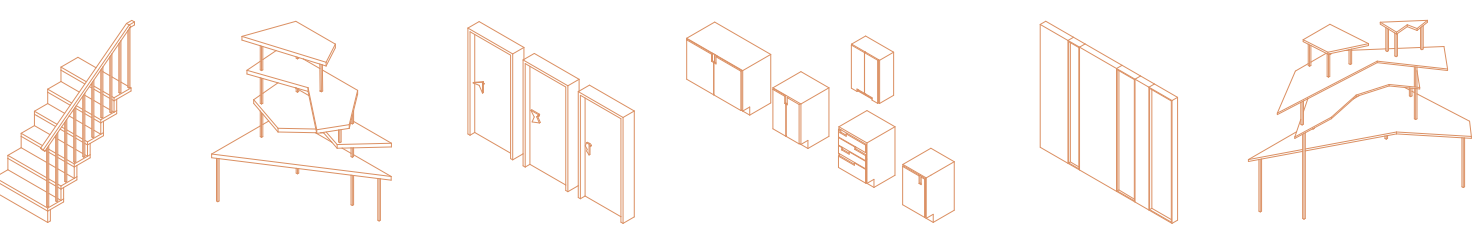




\section{THE FLOOR PLAN DEVELOPMENT}

\section{STAGE 1}

This is the beginning of the refinement of the floor plans for the apartments in Iteration 3.

At this stage, no walls have been removed or changed. This is simply the results of Phase 4 of The Disturbance process.

LEVEL 1

$1: 200$

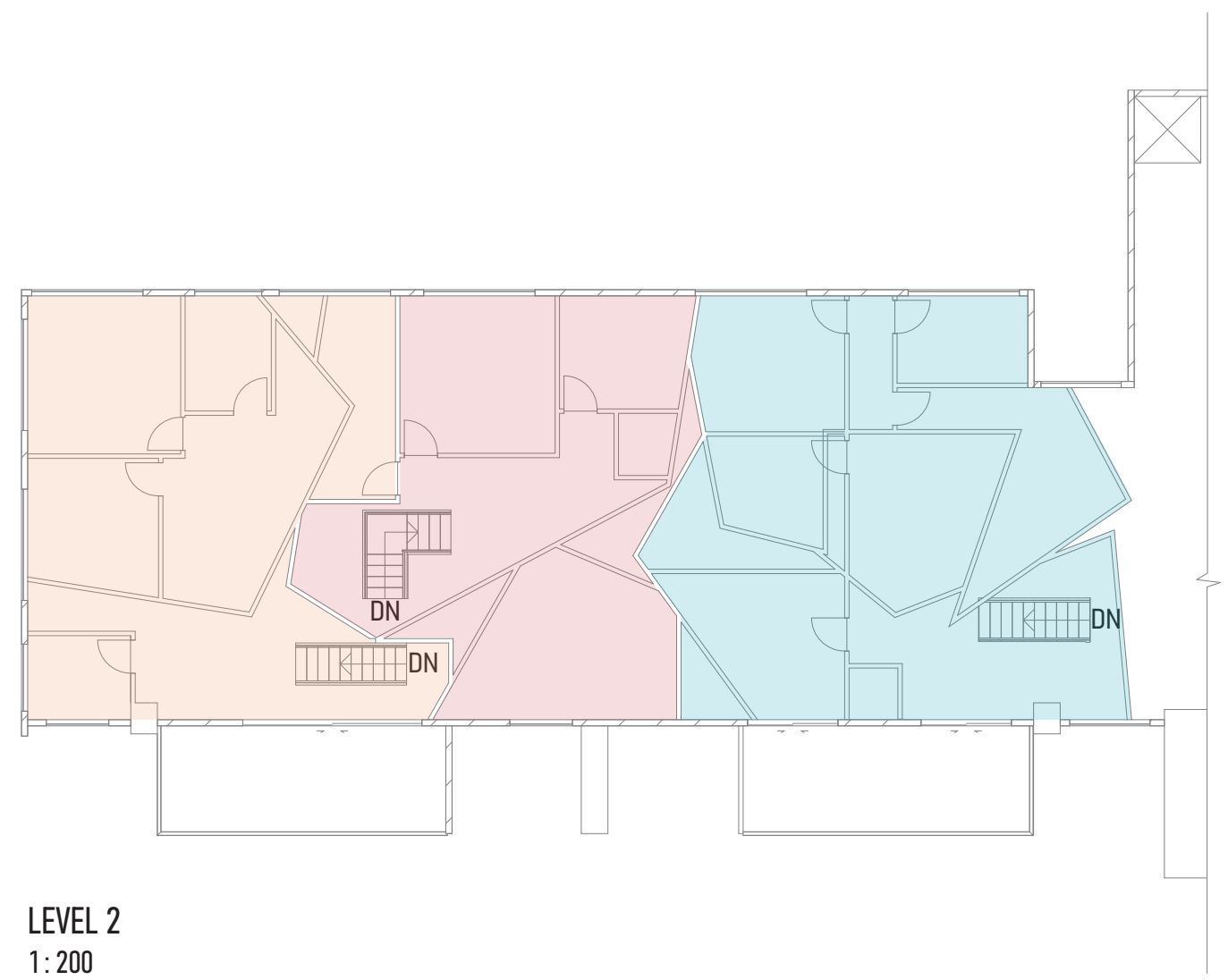




\section{STAGE 2}

This is where the changing of the layout begins. Starting to consider where to remove walls and relocate walls to achieve more functional spaces whilst not losing the angular aesthetic.

One of the first things which becomes a mandatory change to achieve this is the shape of the stairs as well as their location.

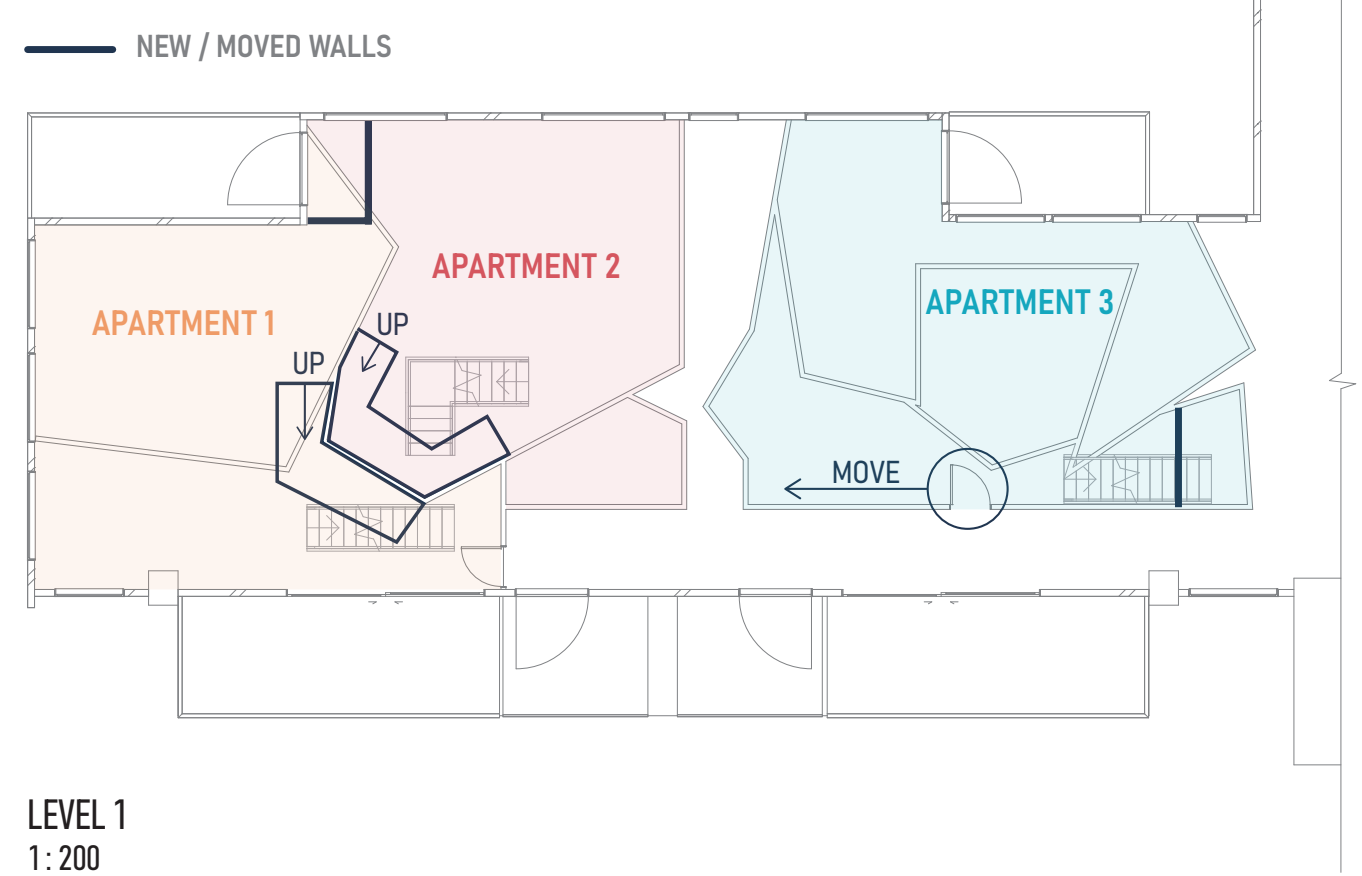

LEVEL 1
$1: 200$

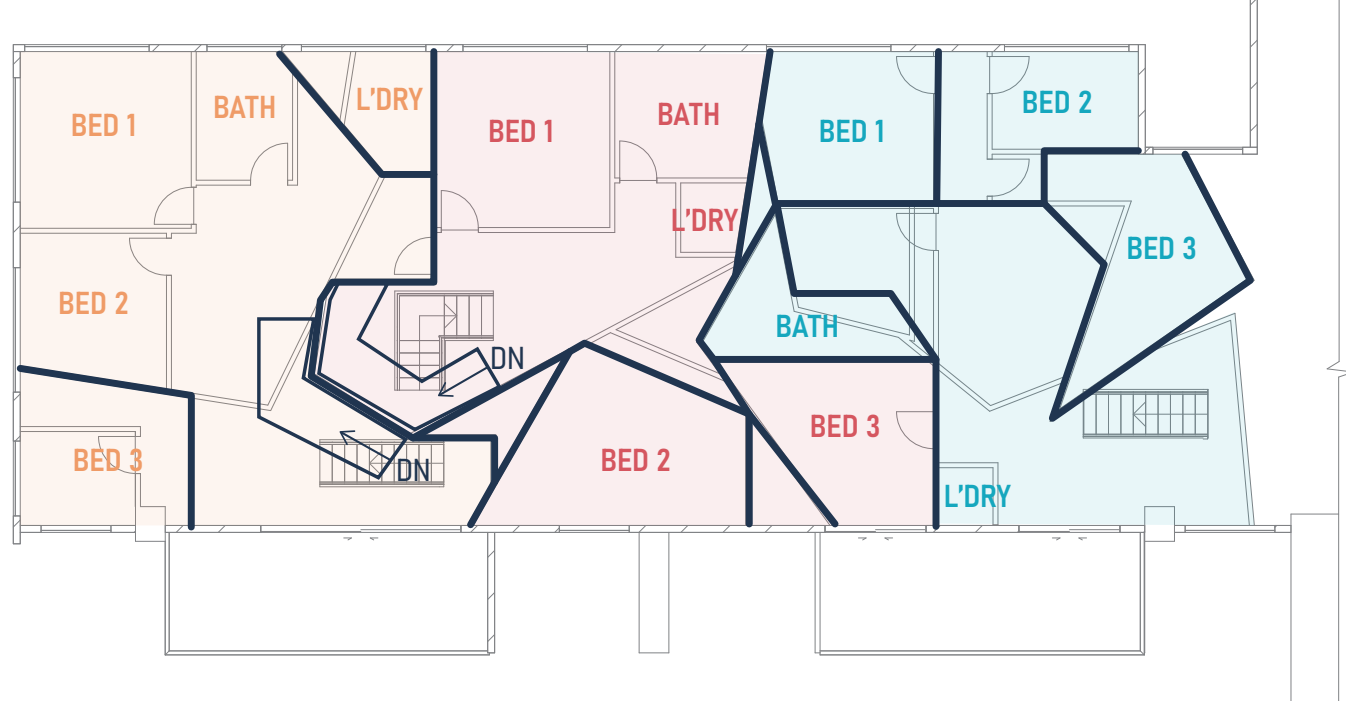

LEVEL 2

1:200 


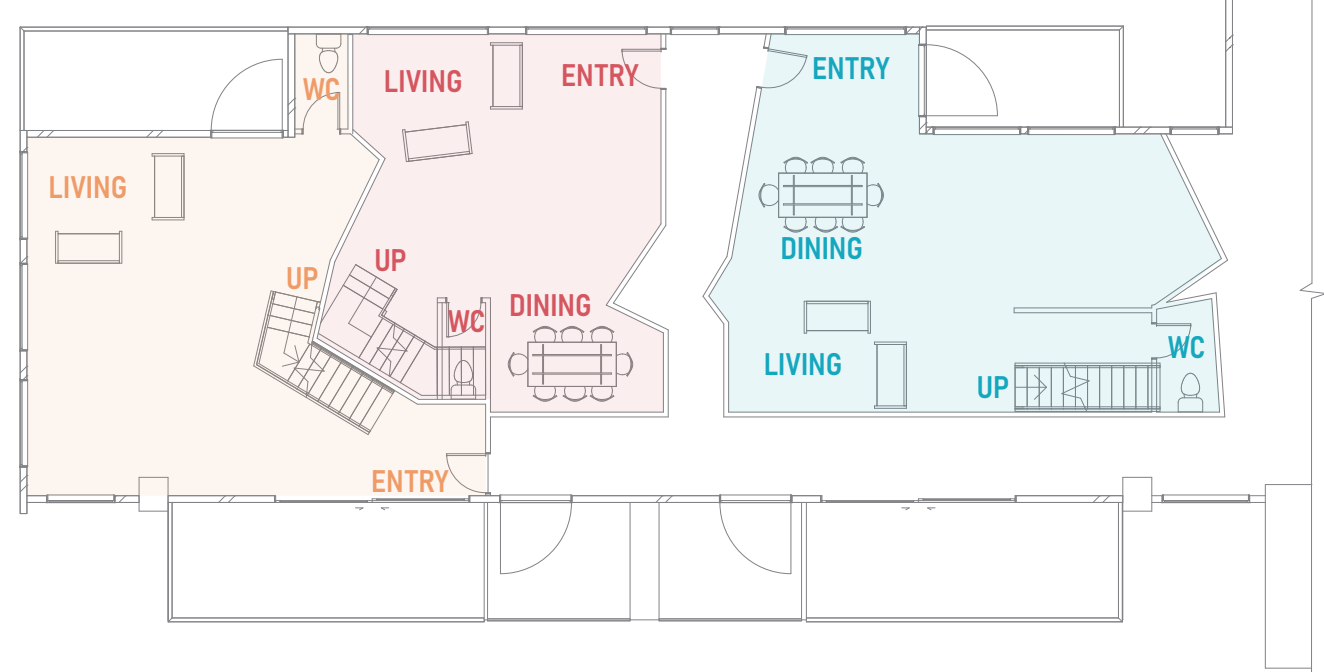

\section{STAGE 3}

At this point, the wall changes developed in Stage 2 are digitally modelled to test their effectiveness and functionality.

Then another level of detail is implemented such as adding furniture and fixtures to gain a better understanding of the scale of the spaces.

The location of doors and storage starts being explored here but there is still need for further consideration.

This stage is mainly focused on the upstairs layout. The following stage focus on finalising this layout as well as fully developing the downstairs layout.

LEVEL 1

1:200

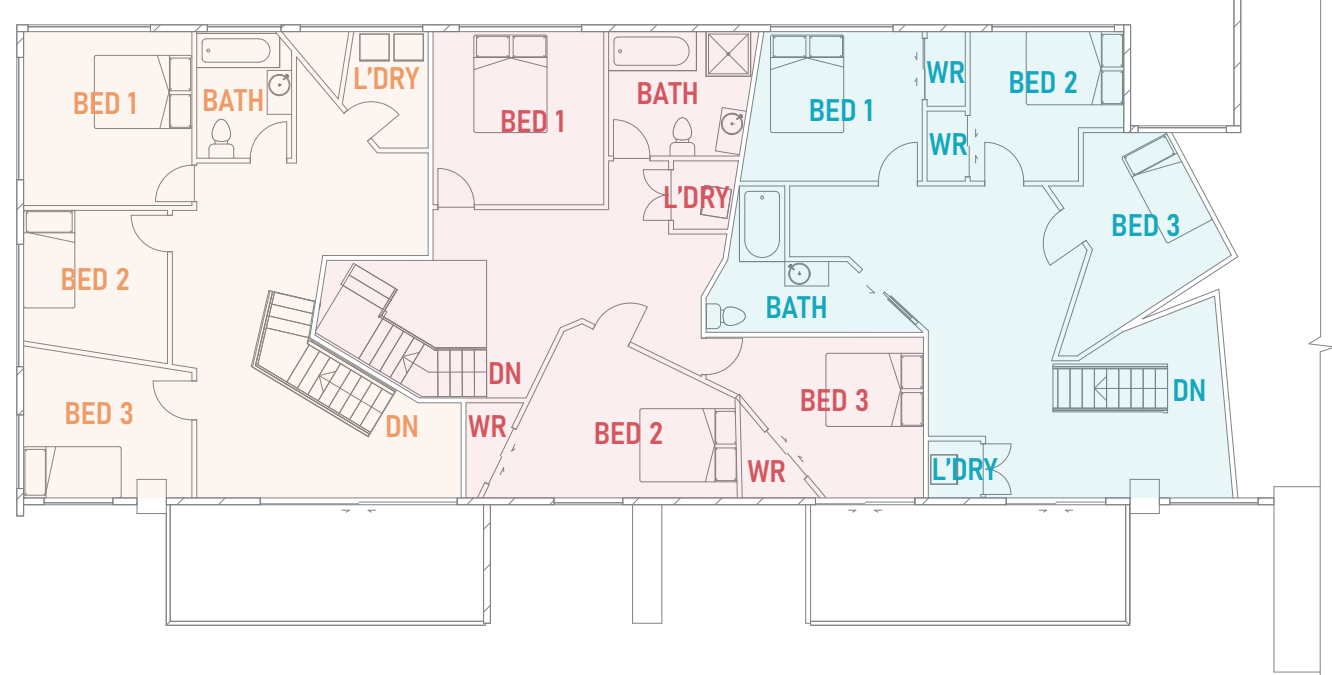

LEVEL 2

$1: 200$ 


\section{STAGE 4}

At this point the focus is shifted to downstairs and the location as well as the size of the kitchen, living and dining spaces is finalised.

It is important to have a toilet on this lower level as well and so the location of these had to be decided.

The points of entry into the apartments is also further developed with some of them moving to entirely new locations.

The location of storage within every bedroom is finalised and some opportunities for further storage are found throughout.

There are various instances where the design of custom furniture and/or storage need to be implemented.

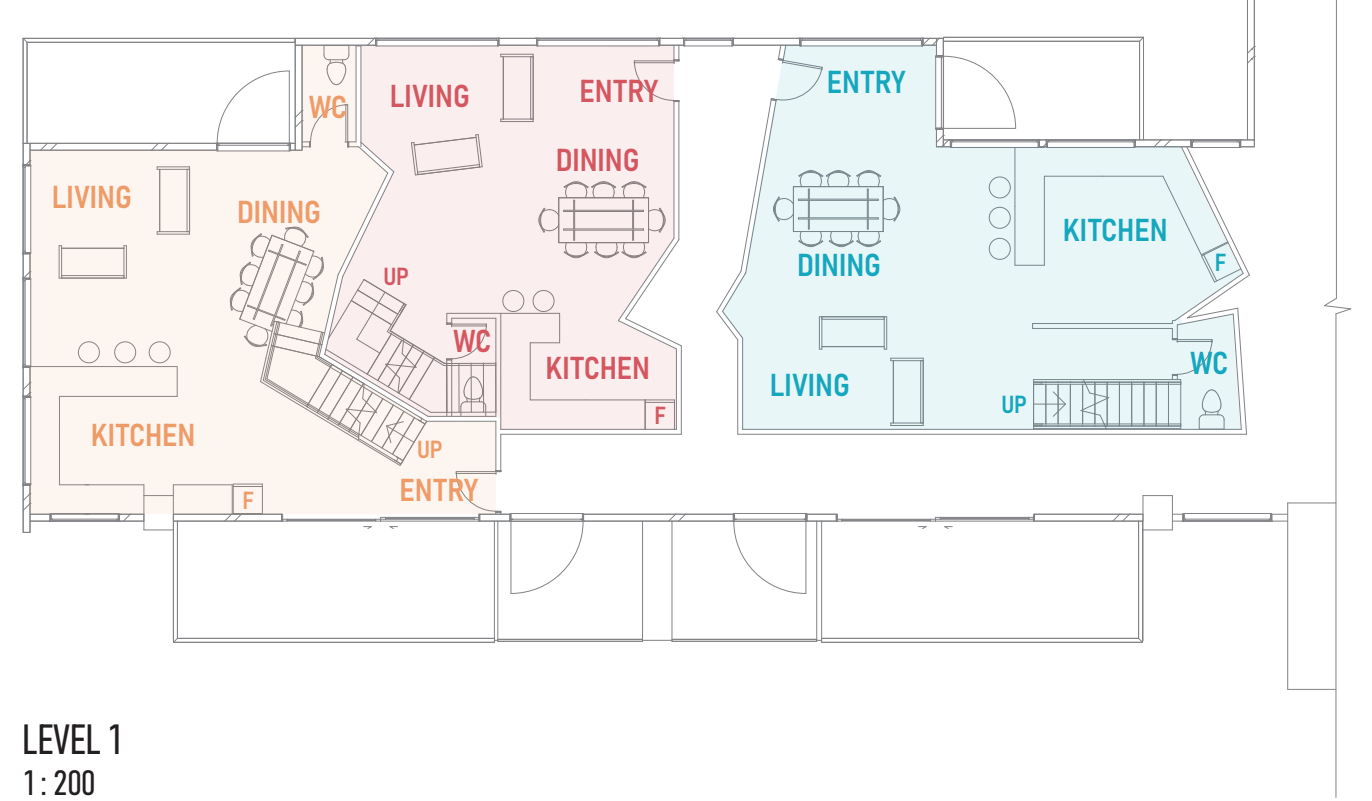

1:200

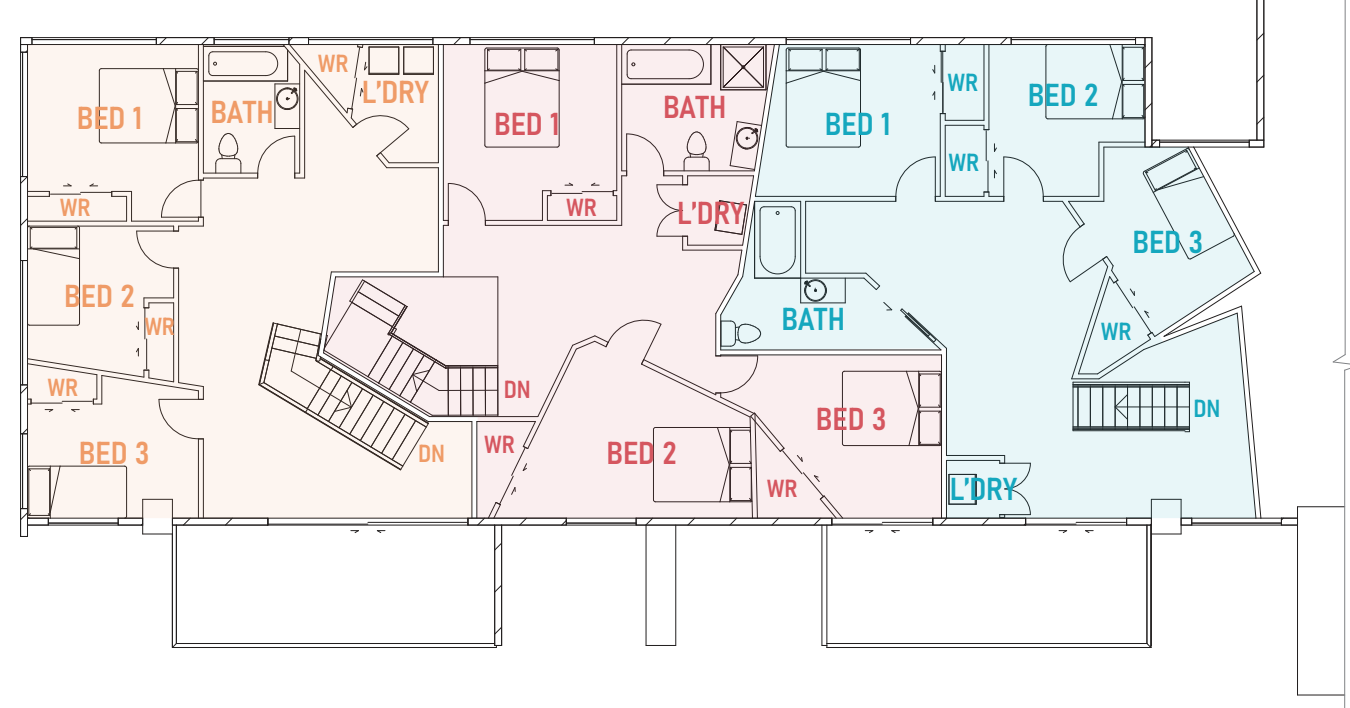

LEVEL 2

$1: 200$ 

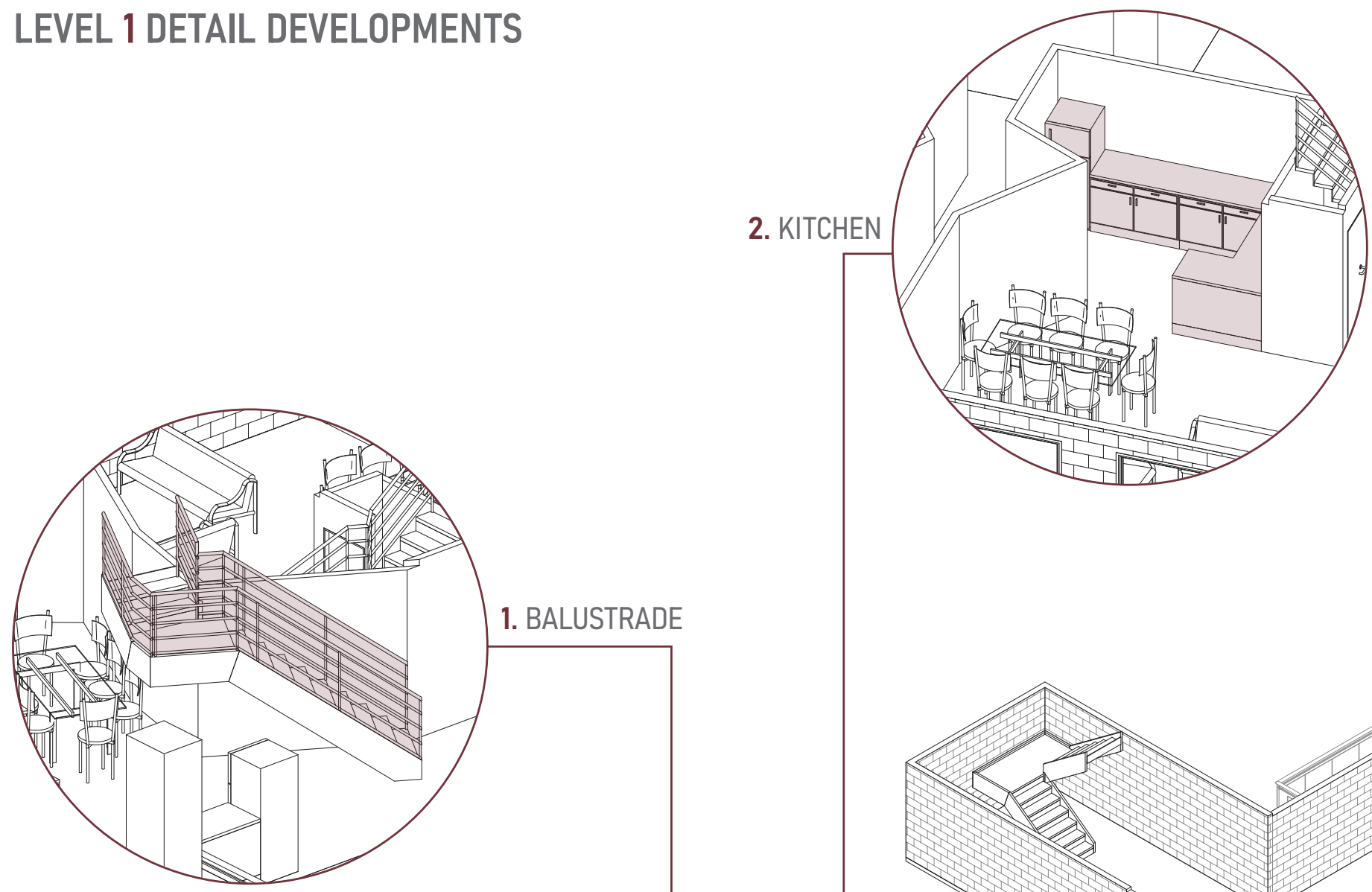

3. PARTITION SCREEN
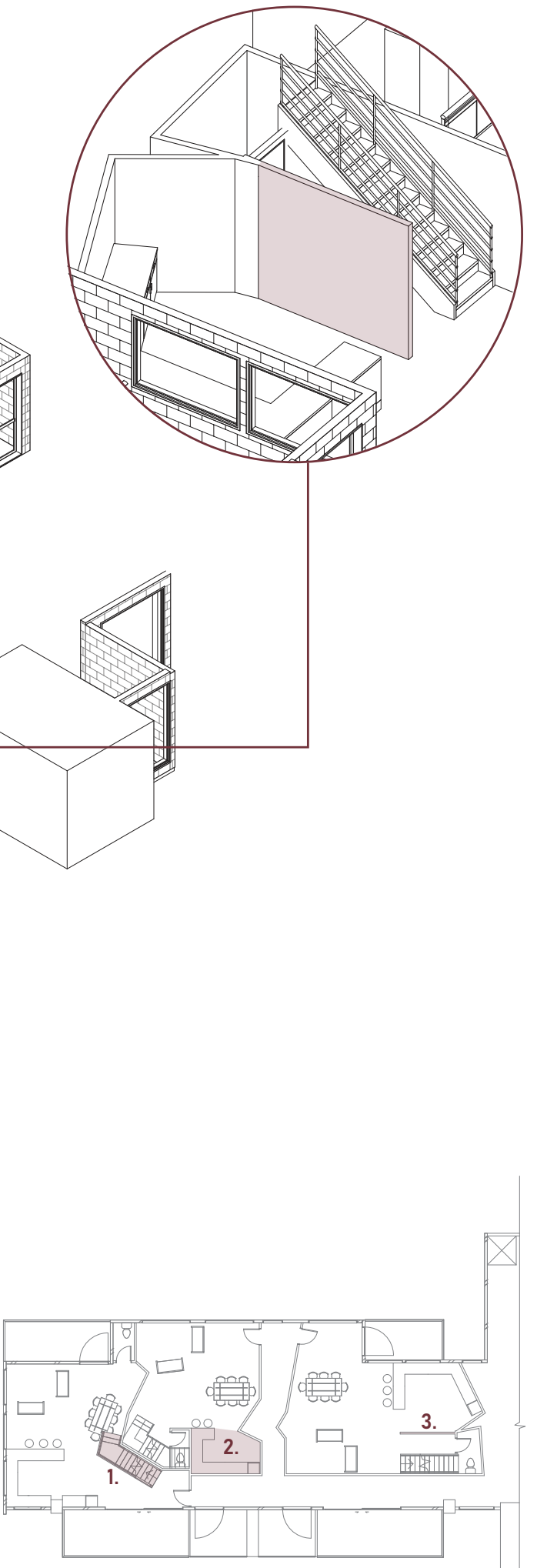

LEVEL 1 


\section{LEVEL 2 DETAIL DEVELOPMENTS}
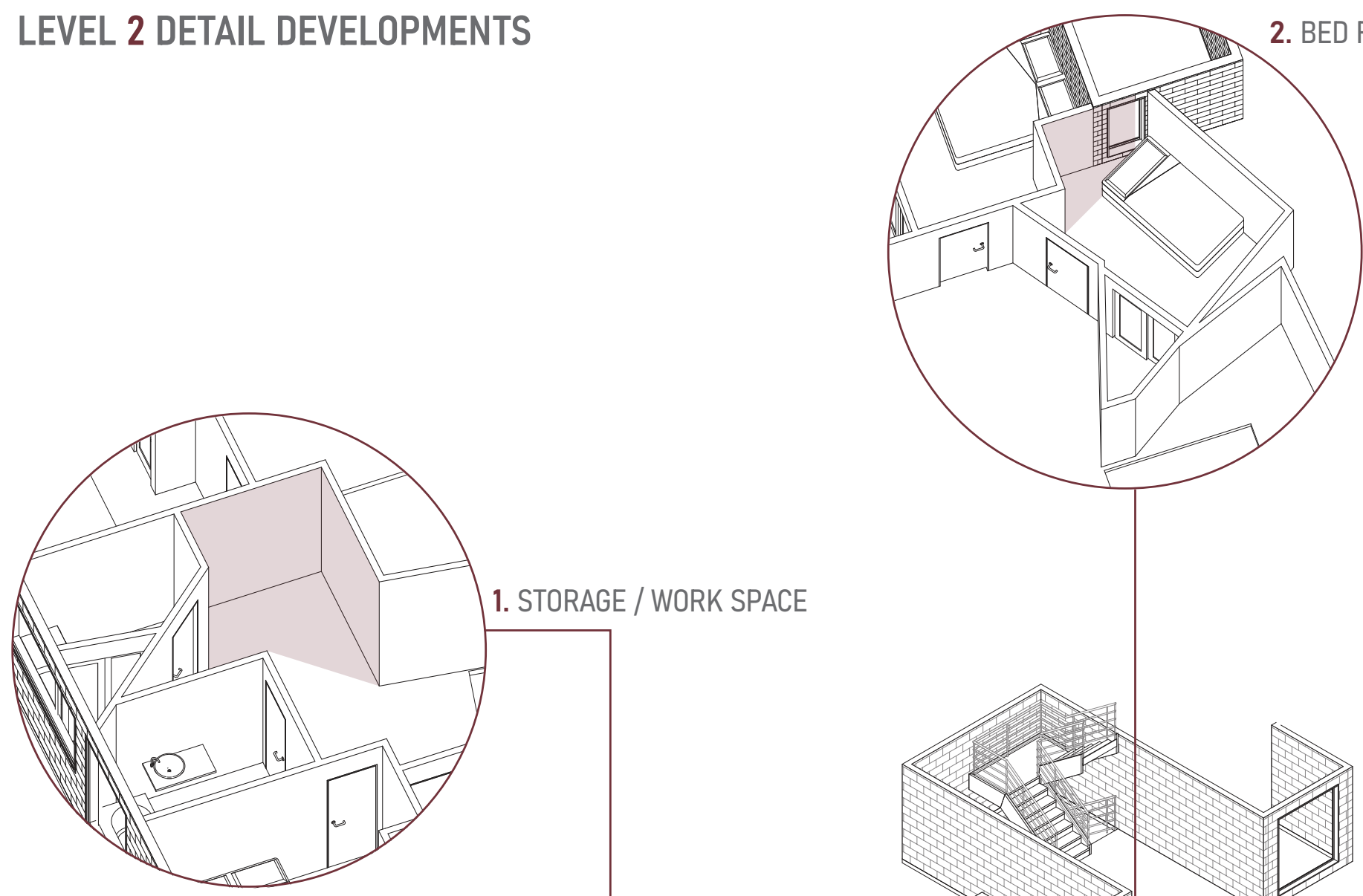

3. DOOR HANDLES + FRAMES

1. STORAGE / WORK SPACE
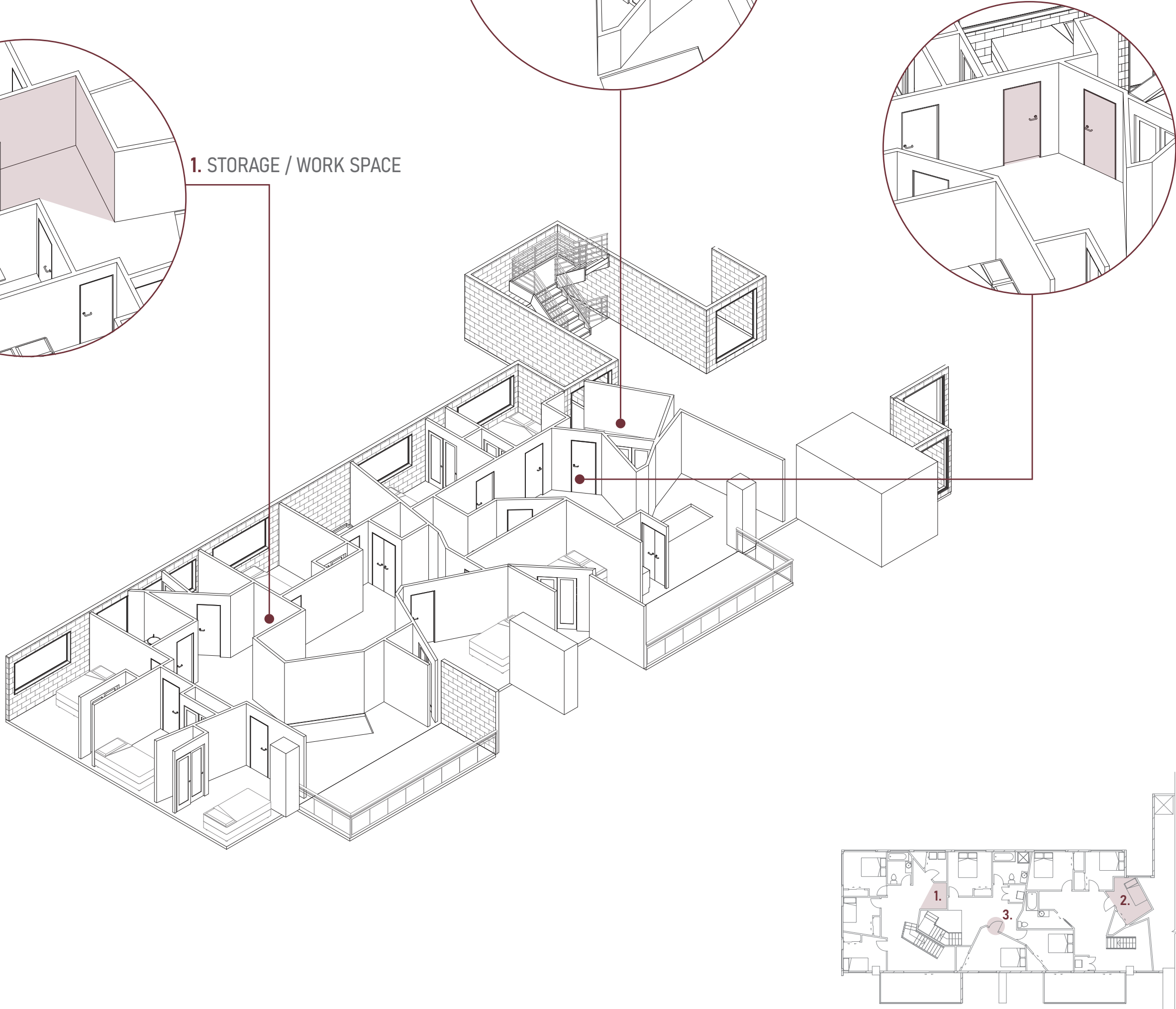


\section{Detailing Precedents}

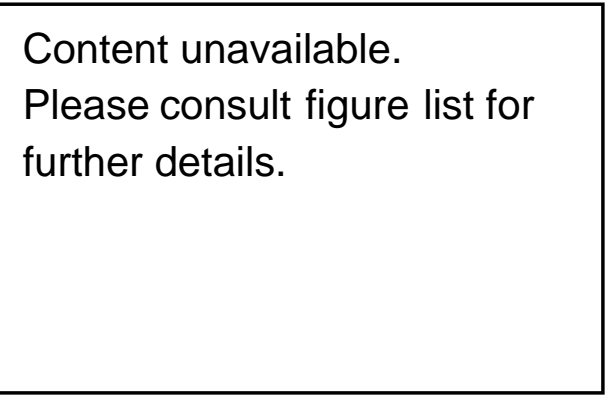

Figure 52

Content unavailable.

Please consult figure list for further details.

Figure 53

\section{Content unavailable.}

Please consult figure list for further details.
House in Hiro,

Suppose Design Office

Content unavailable.

Please consult figure list for further details.

\section{Figure 55}

Content unavailable.

Please consult figure list for further details.

Figure 56

Content unavailable.
Content unavailable.

Please consult figure list for further details.

Figure 58

Content unavailable.

Please consult figure list for further details.

Figure 59

Content unavailable.

Please consult figure list for further details.

Figure 60

\section{El Pueblito Paisa,}

Medellin, Colombia
Please consult figure list for further details.

\section{Figure 60}

Andradas Apartment, OCRE Arquitectura 
AA8 Small House,

Branco-DelRio Arquitectos

Long Hous Apartment,

Talita Nogueira Arquitetura

Content unavailable.

Please consult figure list for further details.

Figure 61

Content unavailable.

Please consult figure list for further details.

Figure 62

Content unavailable.

Please consult figure list for further details. further details.
Content unavailable.

Please consult figure list for further details.

Figure 64

Content unavailable.

Please consult figure list for

Figure 65

Content unavailable.

Please consult figure list for further details.

Figure 66
Content unavailable.

Please consult figure list for further details.

Figure 67

Content unavailable.

Please consult figure list for further details.

Figure 68

Content unavailable.

Please consult figure list for further details.

Figure 69

Nakasone House,

Escobedo Soliz 


\section{Detailing Precedent Analysis}
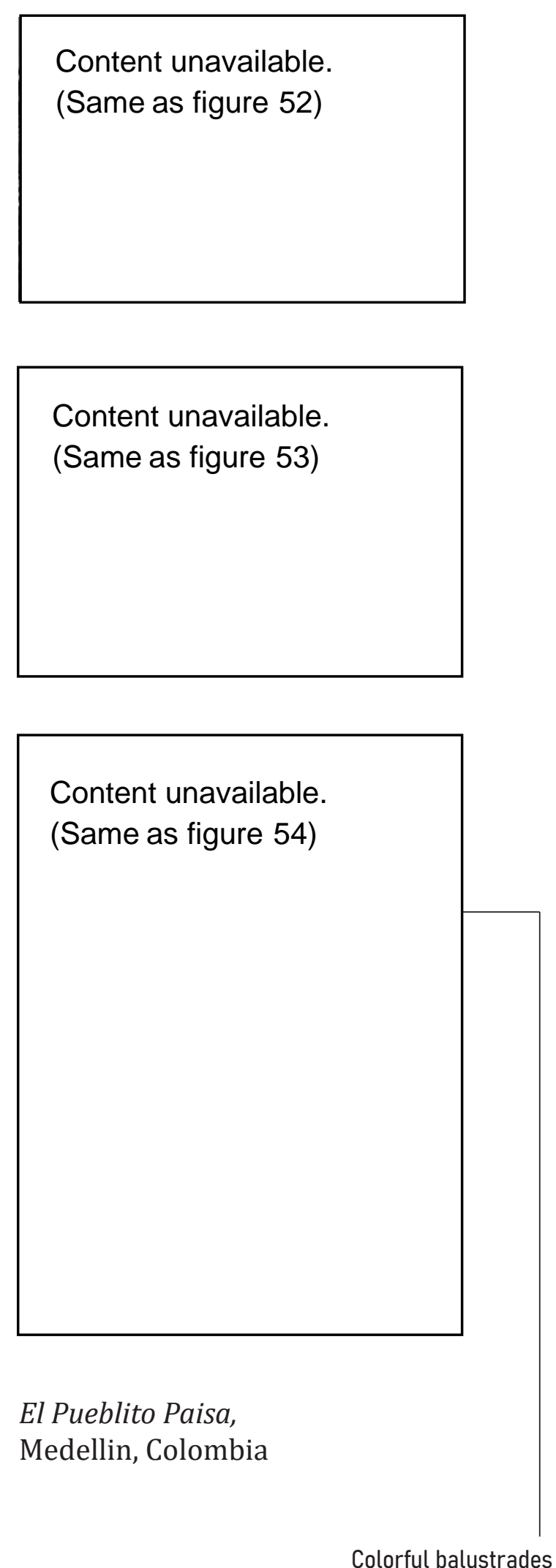

\section{House in Hiro,}

Suppose Design Office

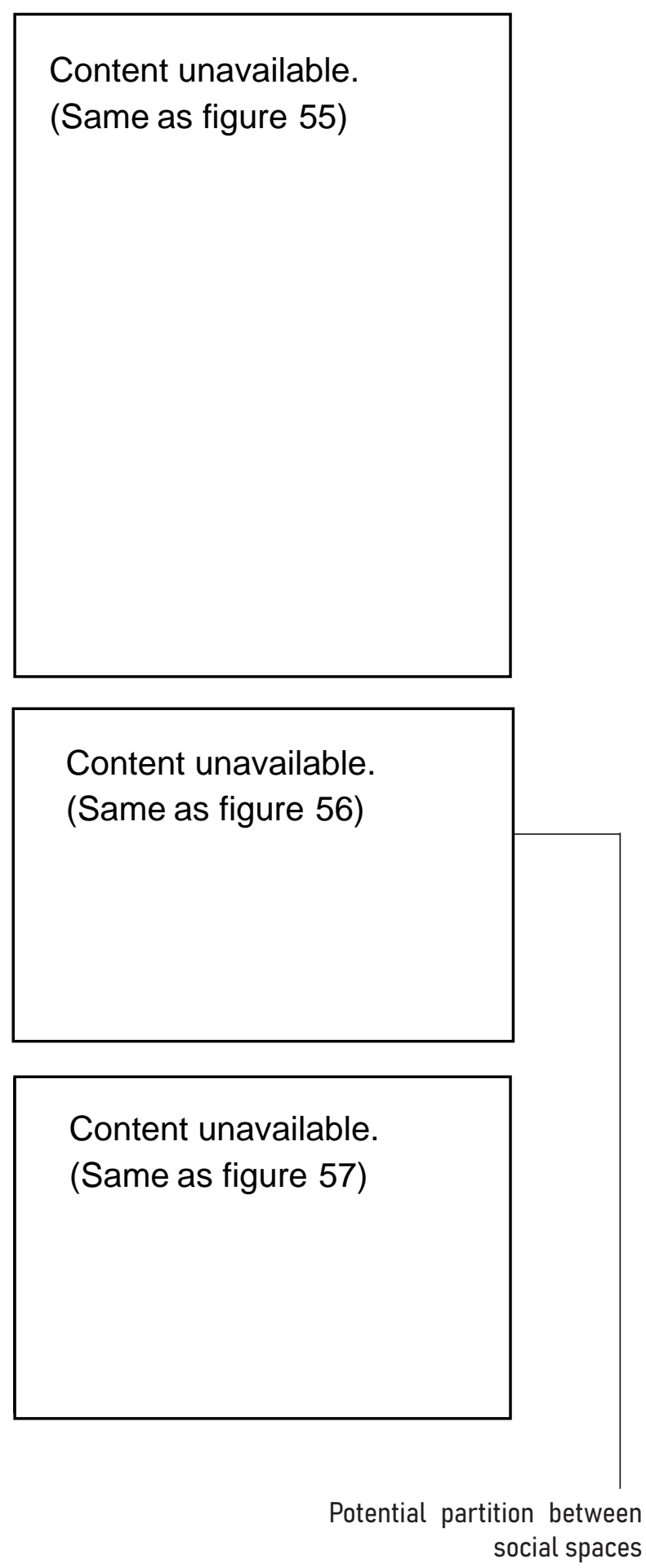

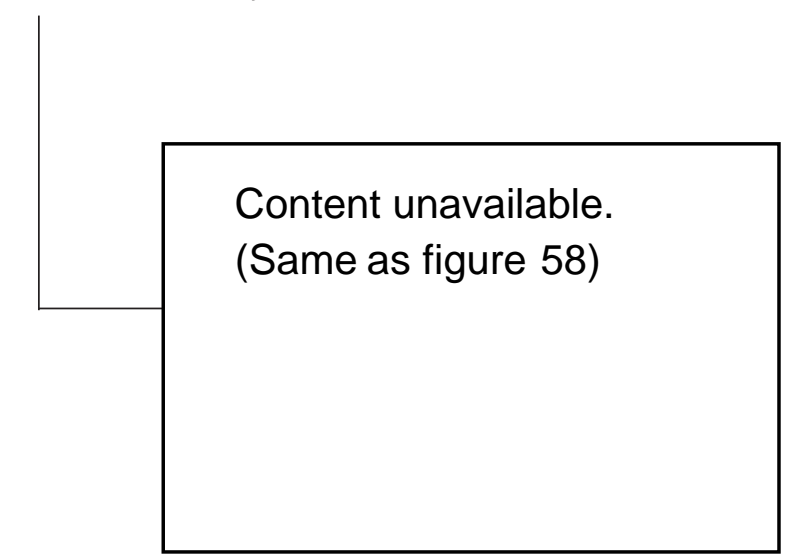

Content unavailable.

(Same as figure 59)

Content unavailable.

(Same as figure 60)

Andradas Apartment, OCRE Arquitectura 
Long Hous Apartment,

Talita Nogueira Arquitetura

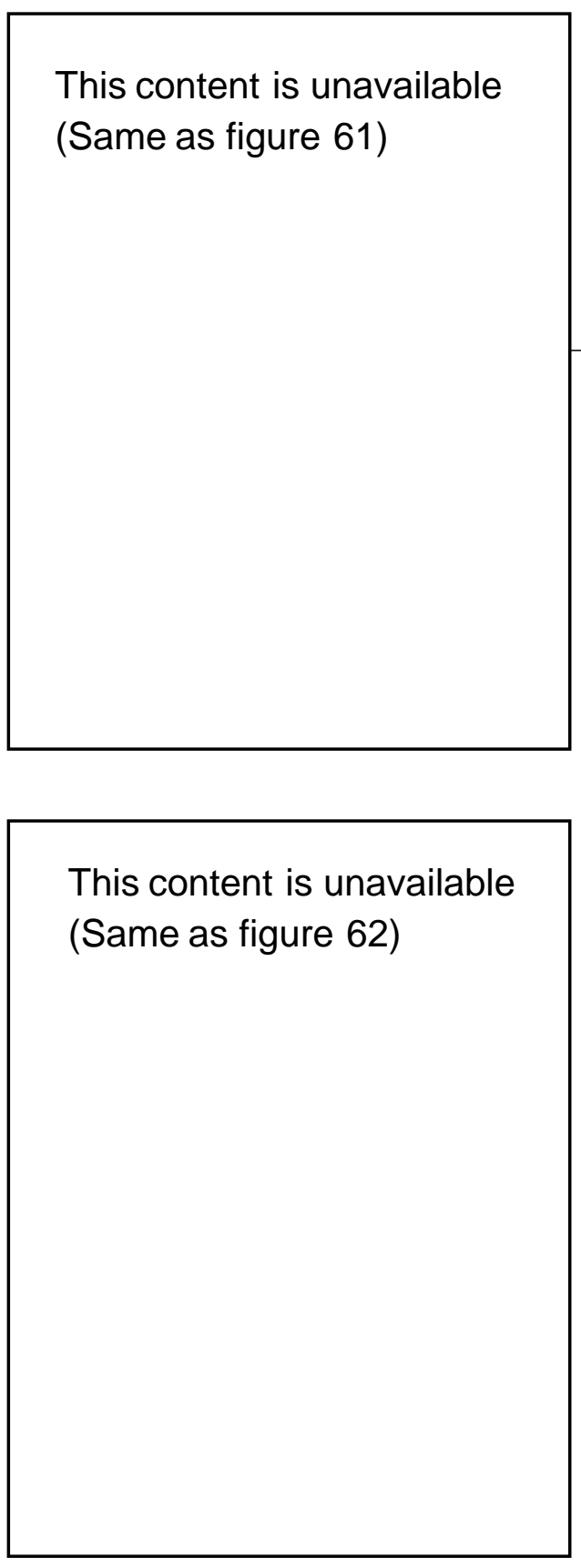

This content is unavailable (Same as figure 63)
AA8 Small House,

Branco-DelRio Arquitectos

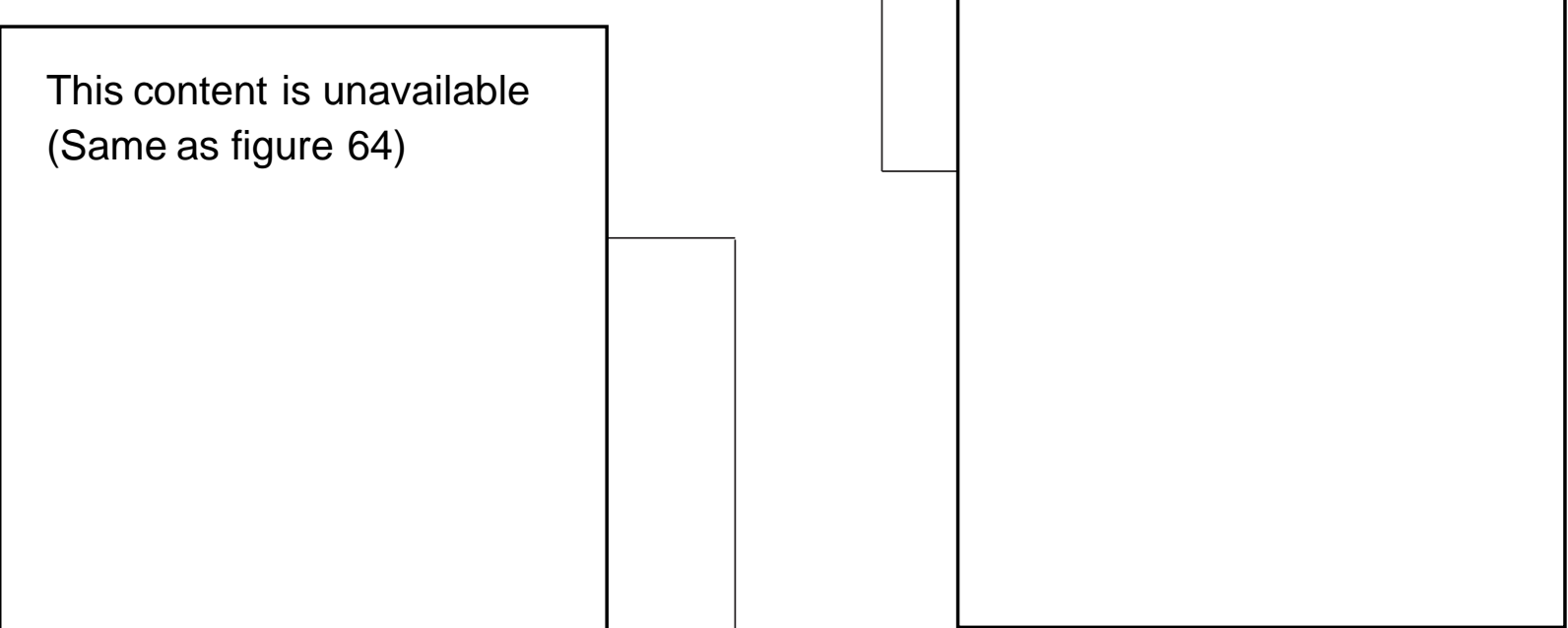

This content is unavailable (Same as figure 65)

This content is unavailable (Same as figure 66)
This content is unavailable

(Same as figure 67)

This content is unavailable (Same as figure 68)
This content is unavailable (Same as figure 69)

Nakasone House,

Escobedo Soliz 
Having looked at these precedents, the process of designing custom furniture and details begins.

It starts with analogue sketching to extract ideas quickly and efficiently. There are six details which are chosen to develop so the conceptualisation is focused around these.

Once this is done, the digital process starts. Each element is modelled digitally and some construction drawings extracted in order to both explore and explain how each element works.

From here the elements are put in place, a final floor plan developed and some final imagery produced to communicate how these objects operate within the apartment space. 
STORAGE /WORK

SPACE

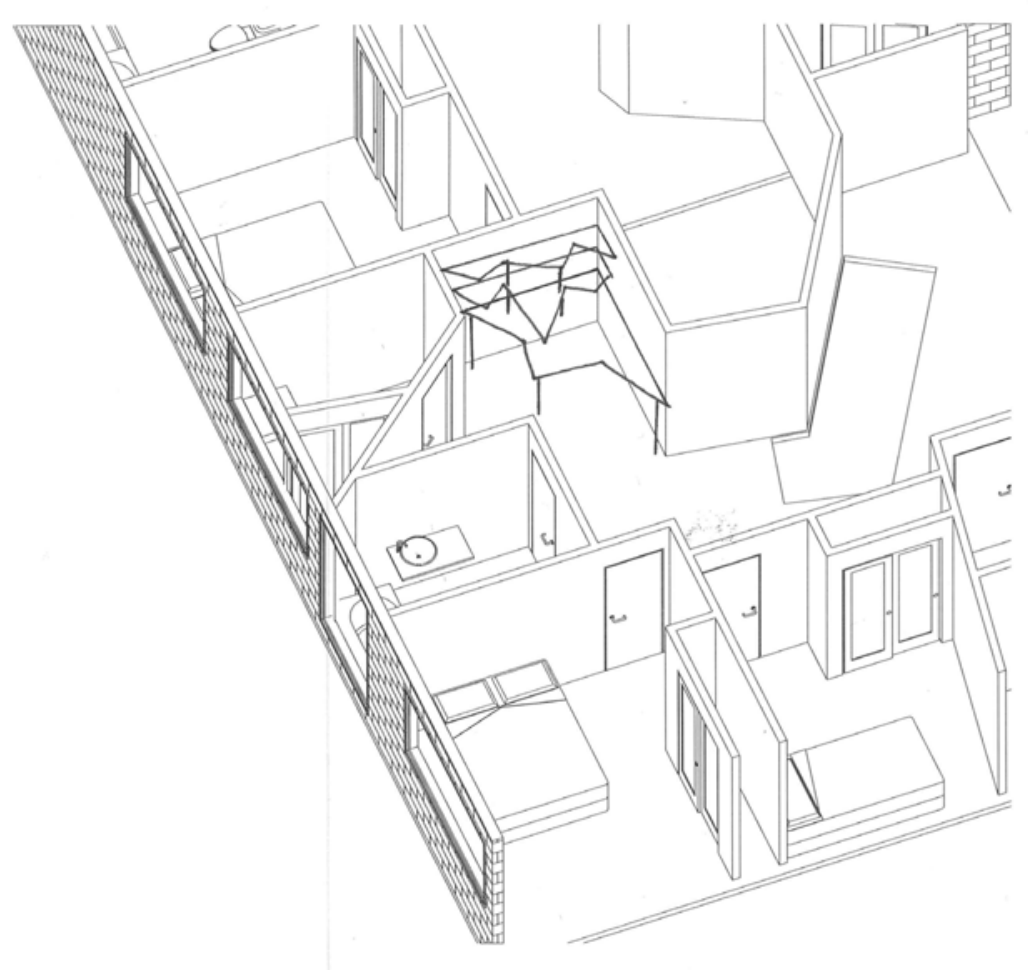

TOP VIEW
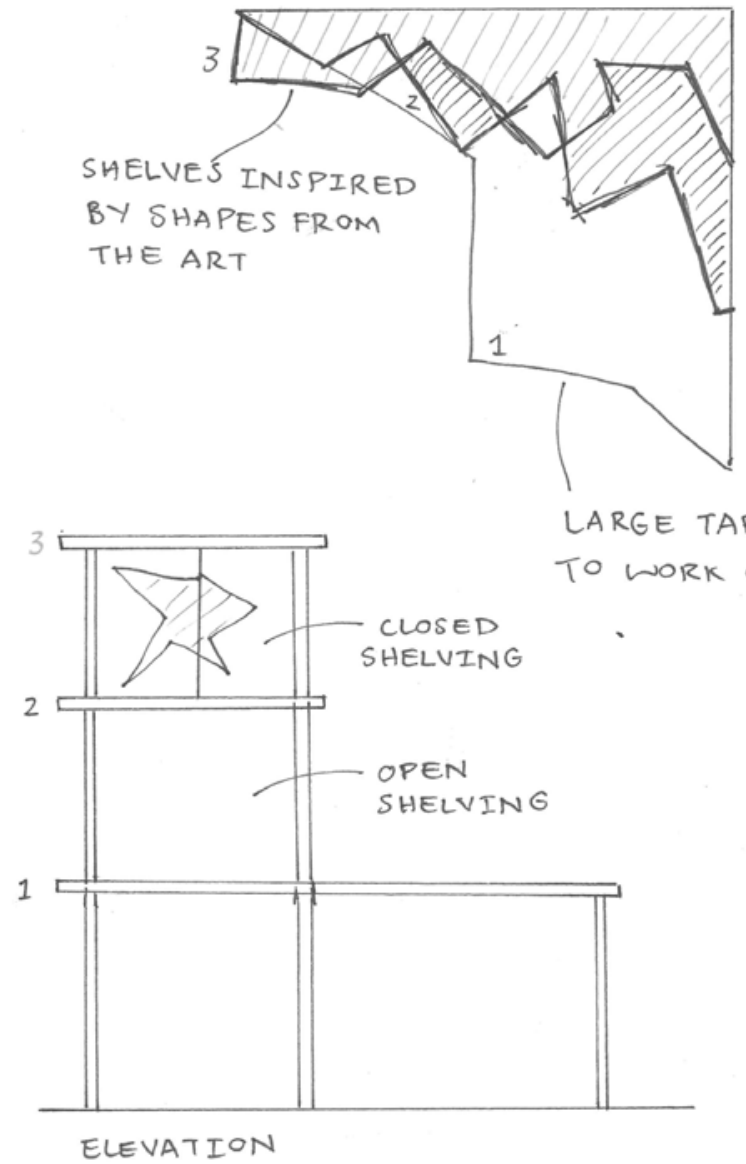

KITCHEN

ALL KITCHENS TC FOLLOW THE SAME GENERAL IDEA. EACH SLIGHTLY DIfFERENT SO ADJUST TO SUIT.
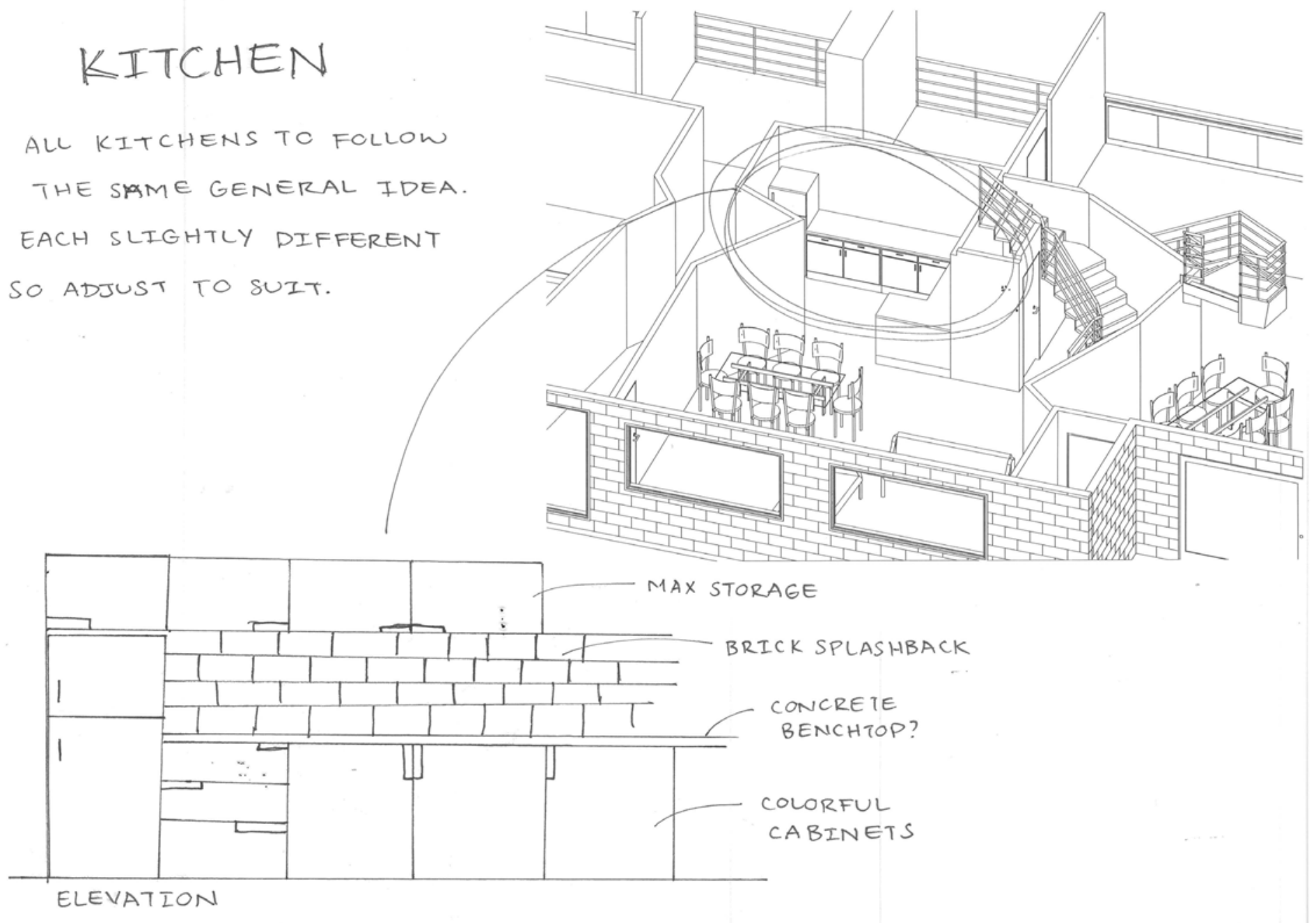

175 


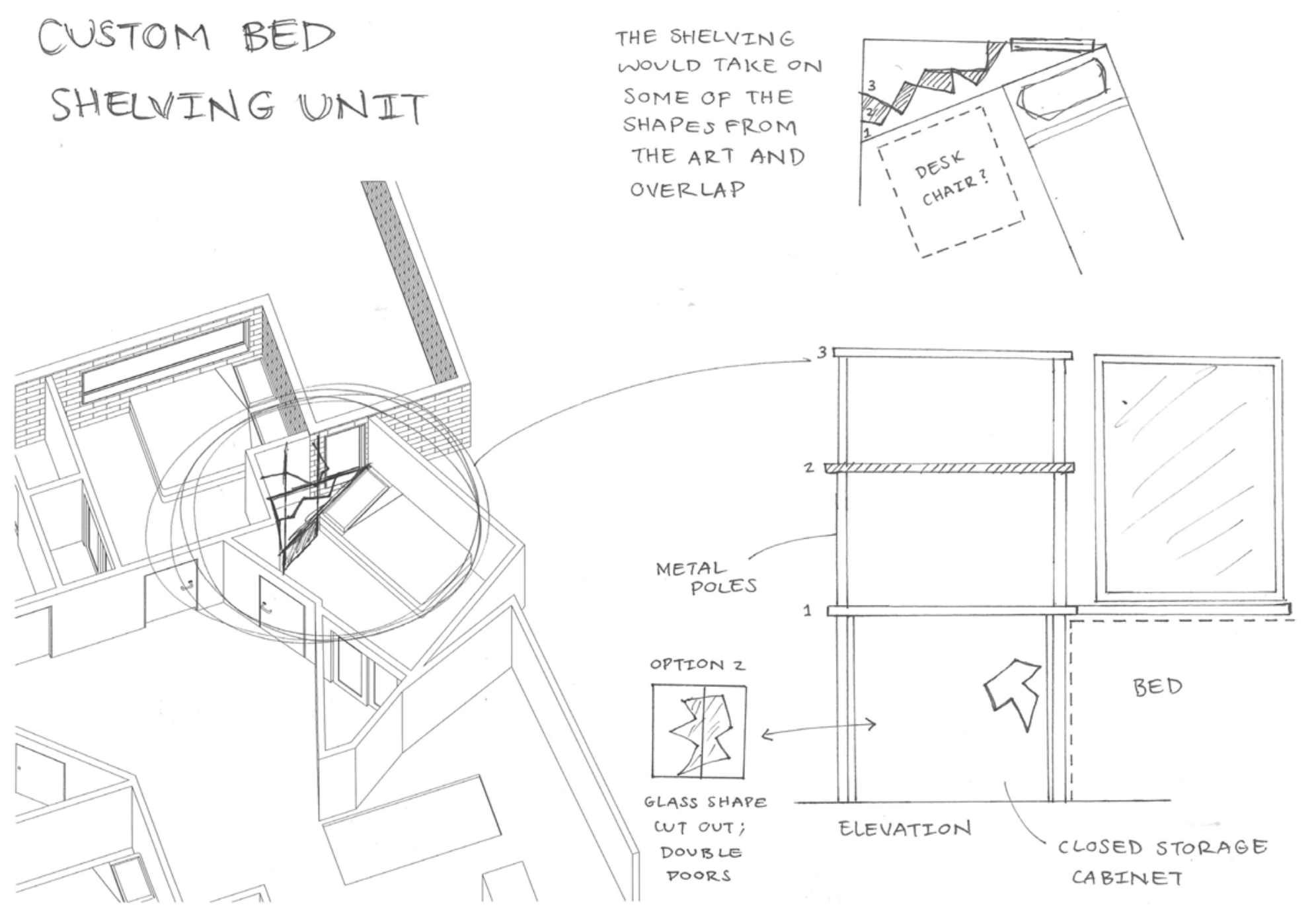

DOOR HANDLE

DOOR HANDLE WOULD BE OFFSET BY METAL BARS

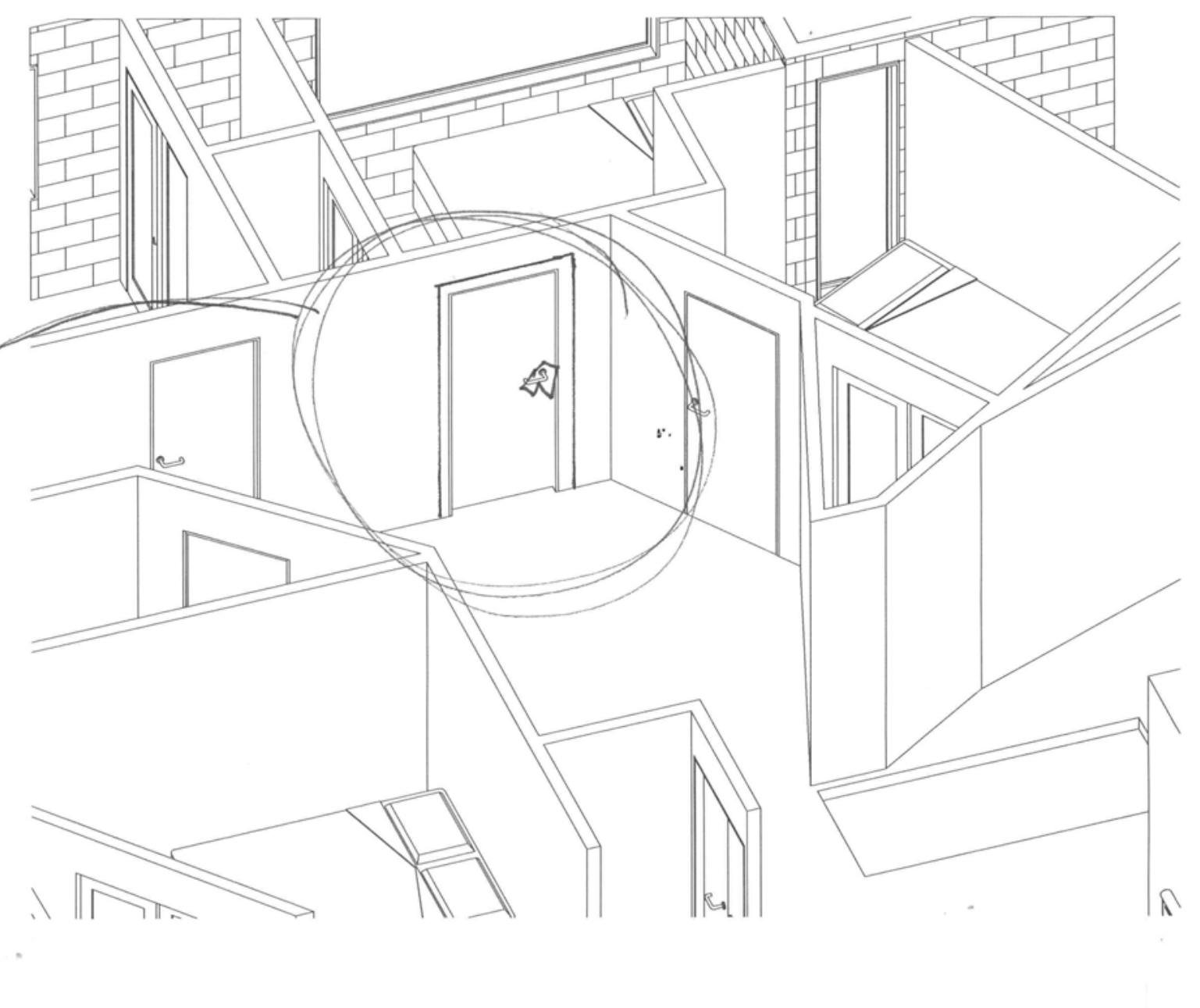




\section{CUSTOM BED SHELVING UNIT}
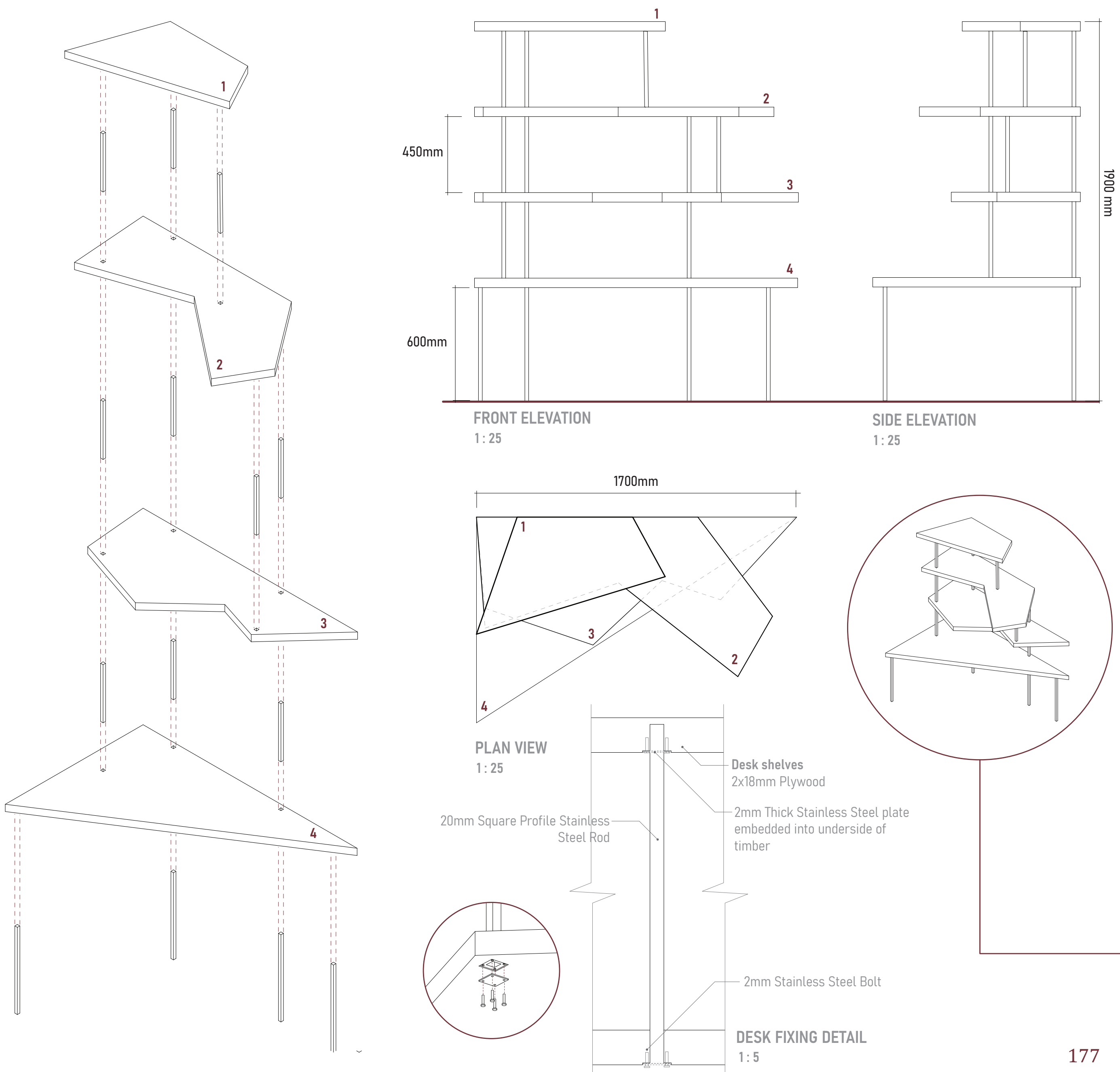
APARTMENT 3 - LEVEL 2

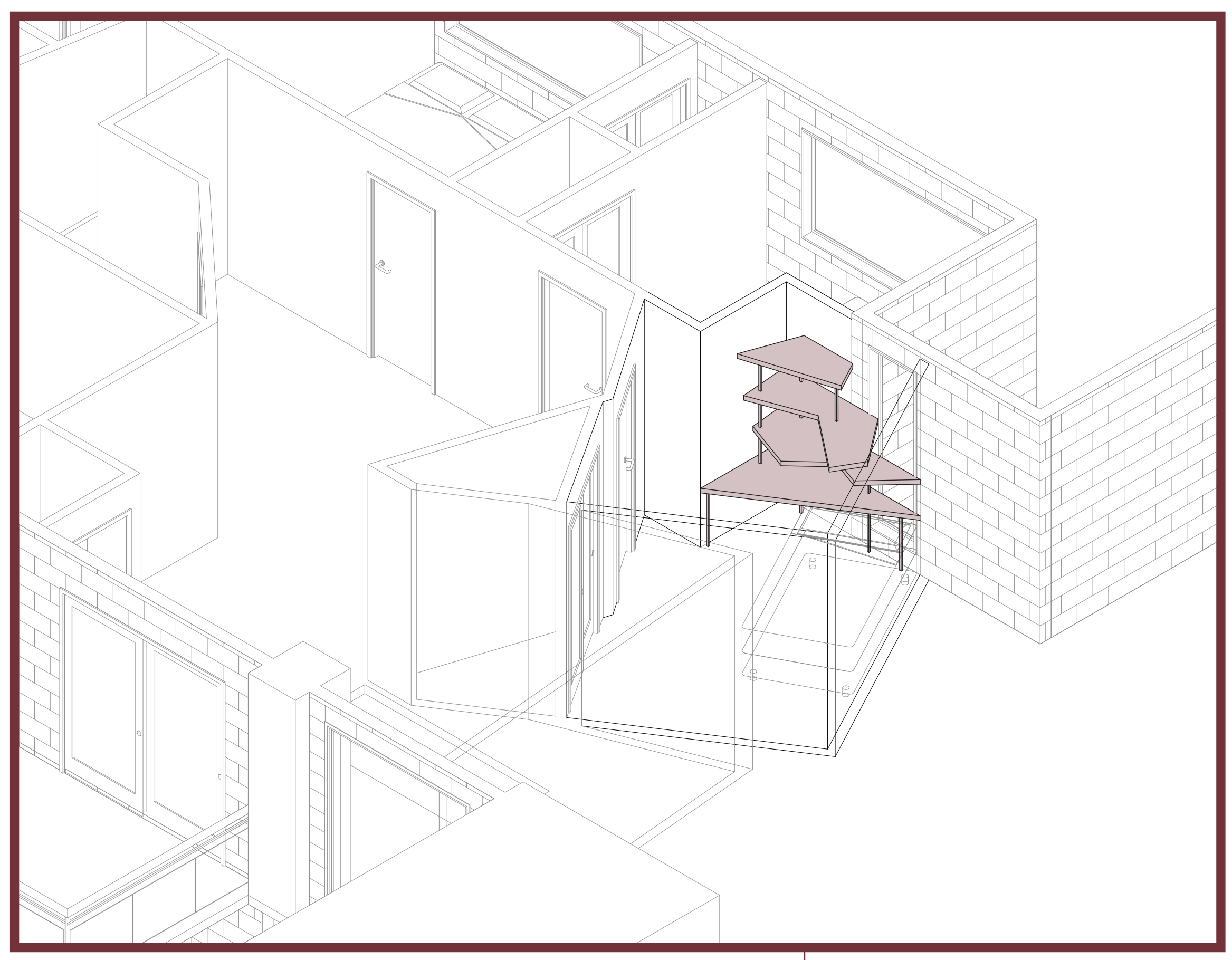




\section{CUSTOM WORKSPACE DESK + SHELVING}
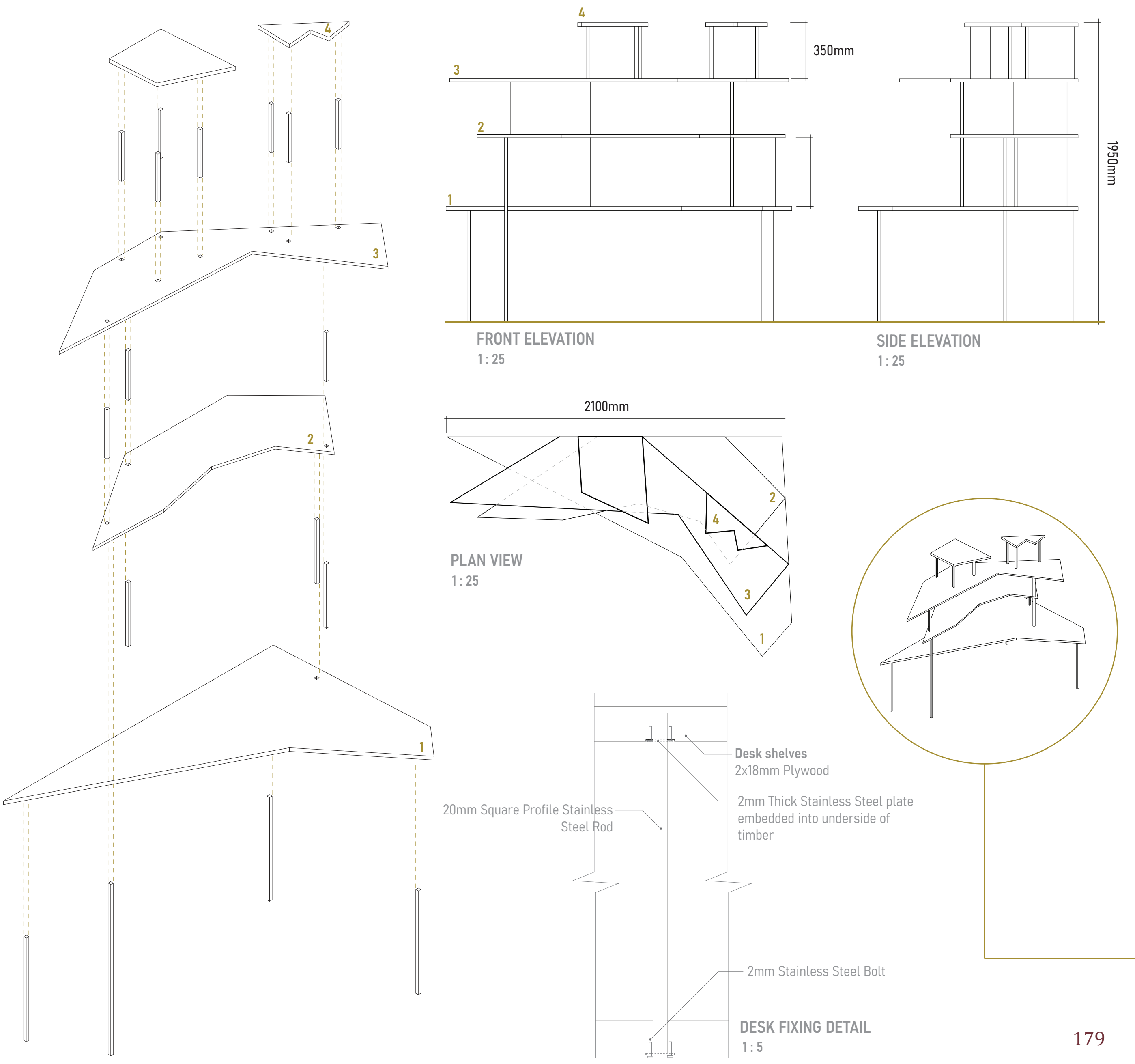


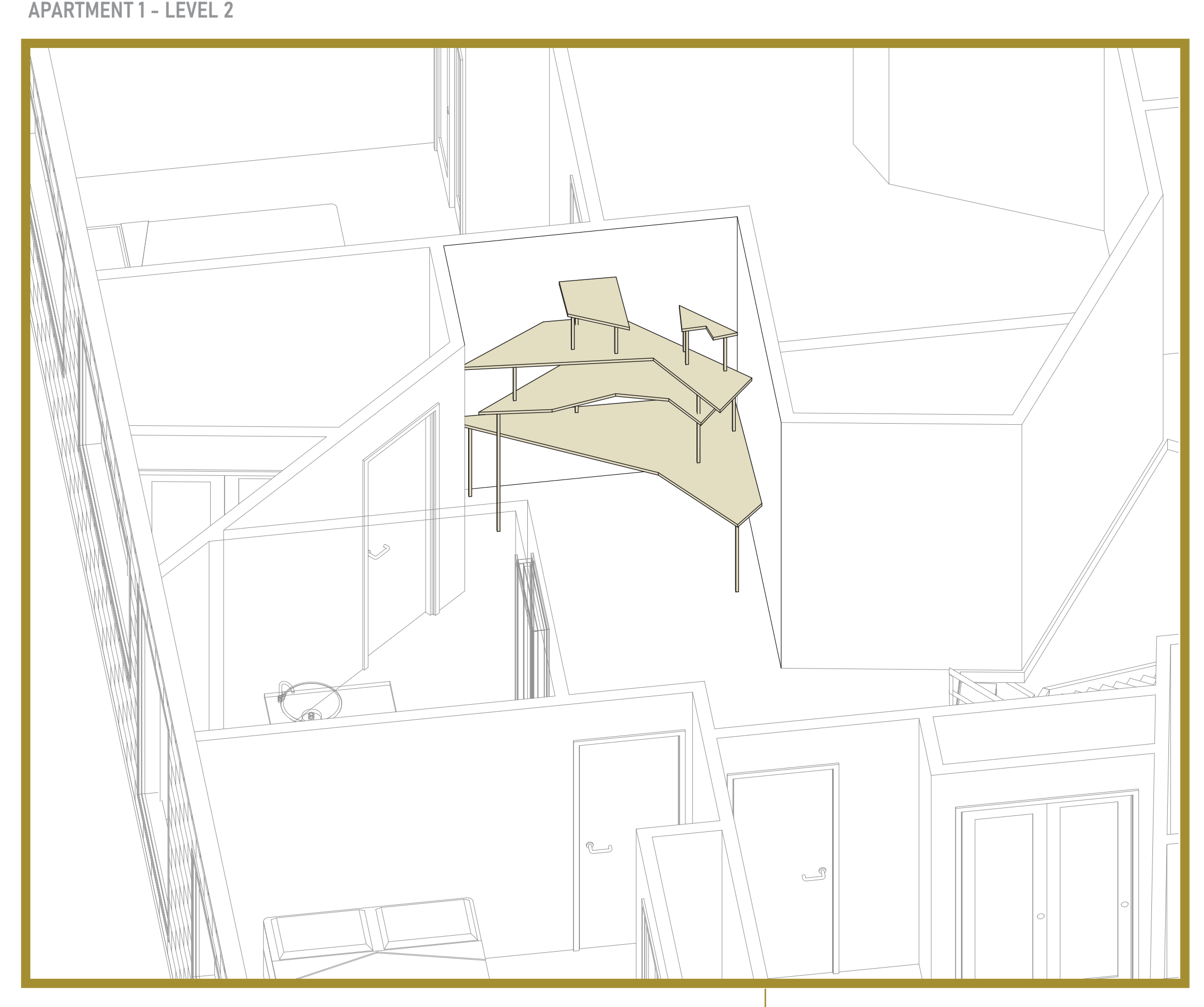




\section{CUSTOM DOOR FRAME + HANDLE}

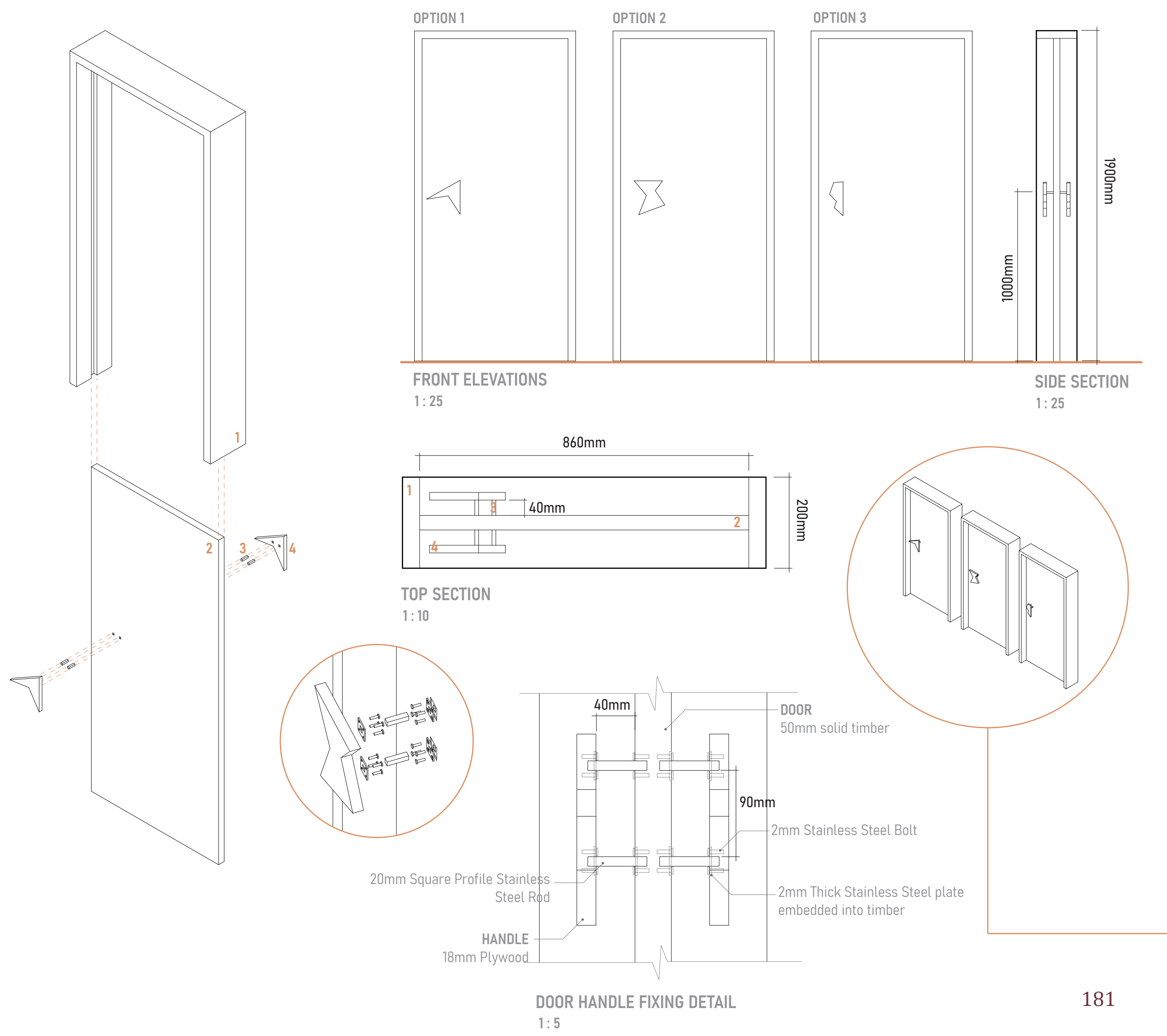




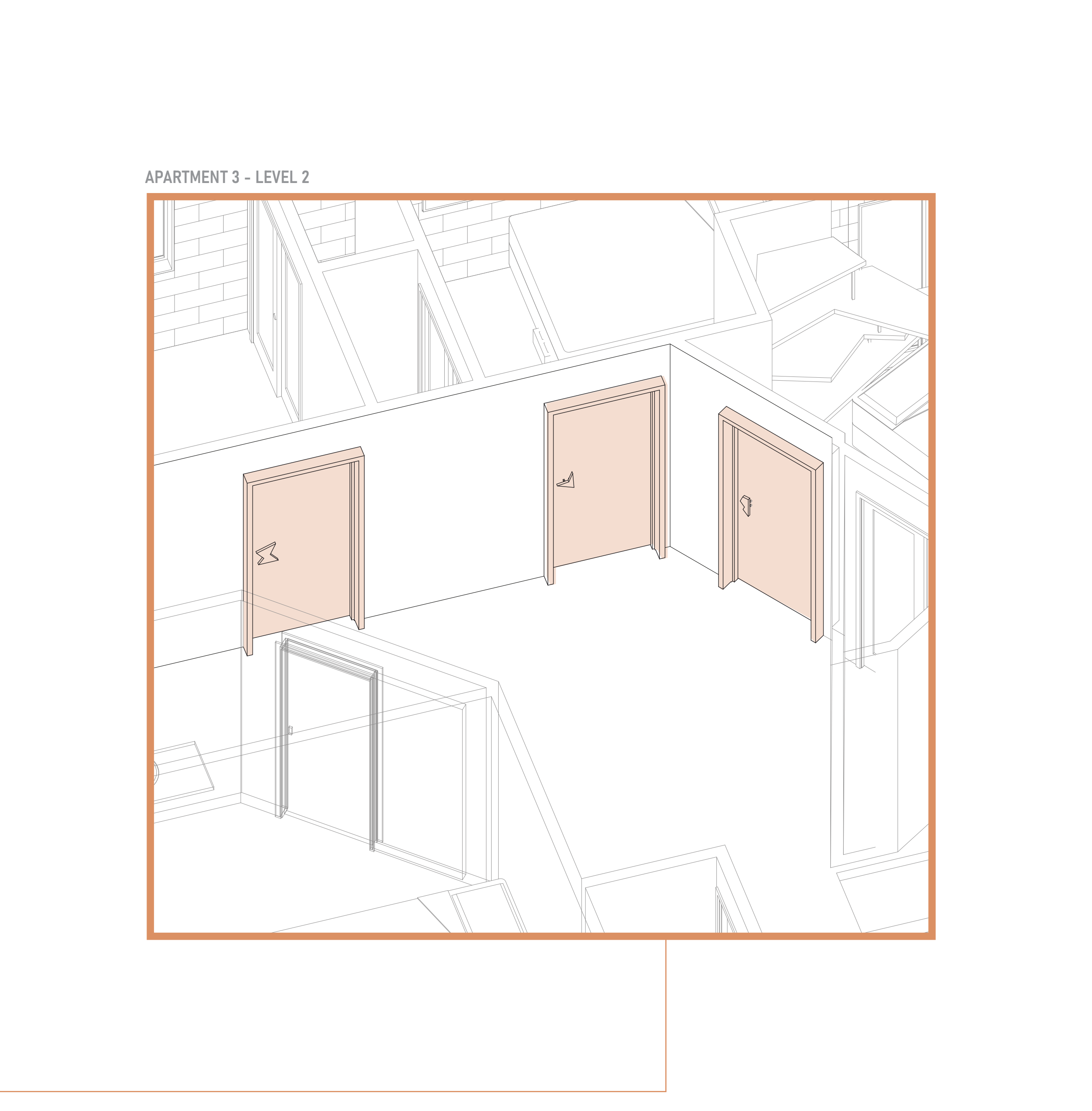$$
\text { A }
$$ 


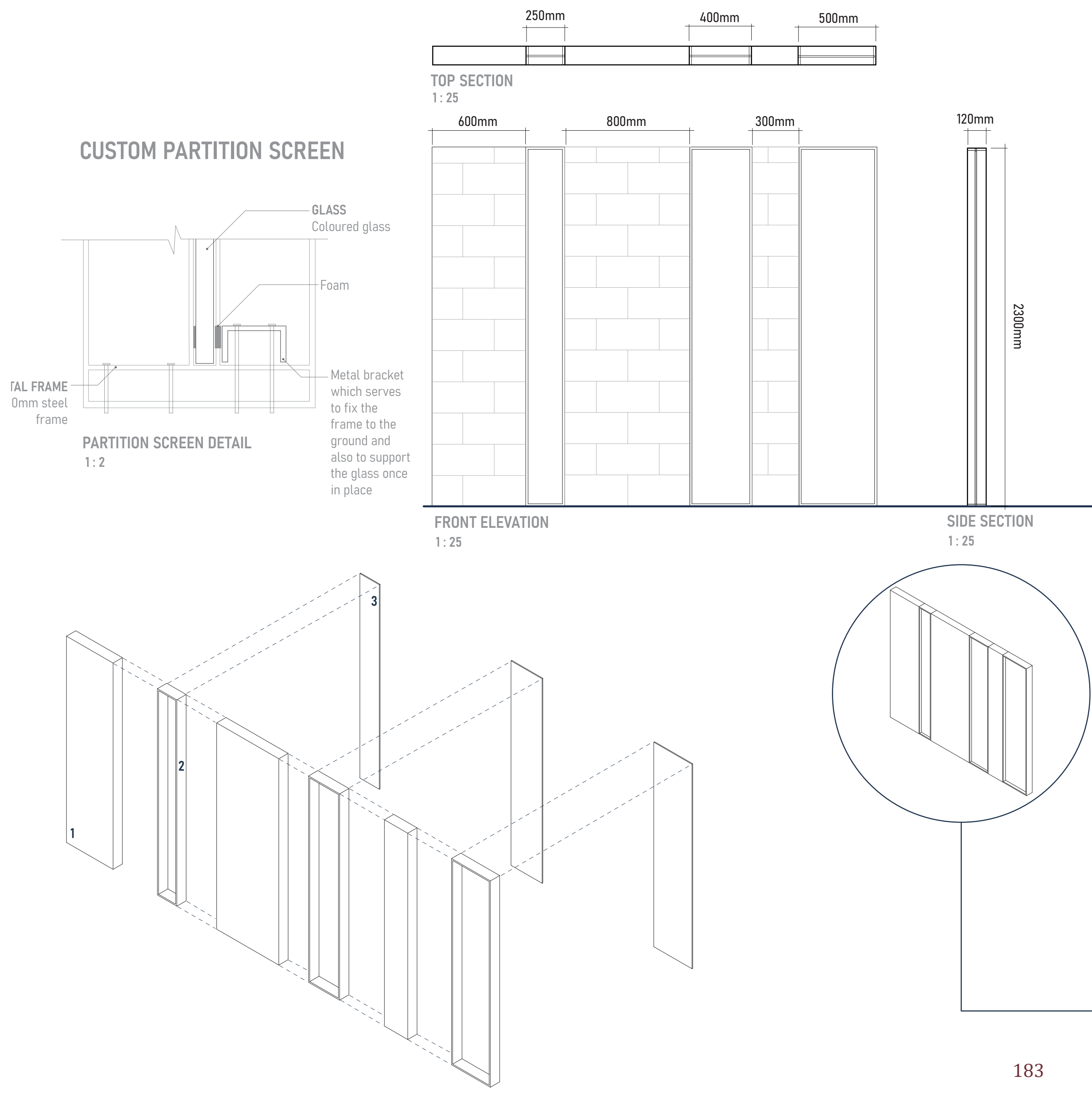




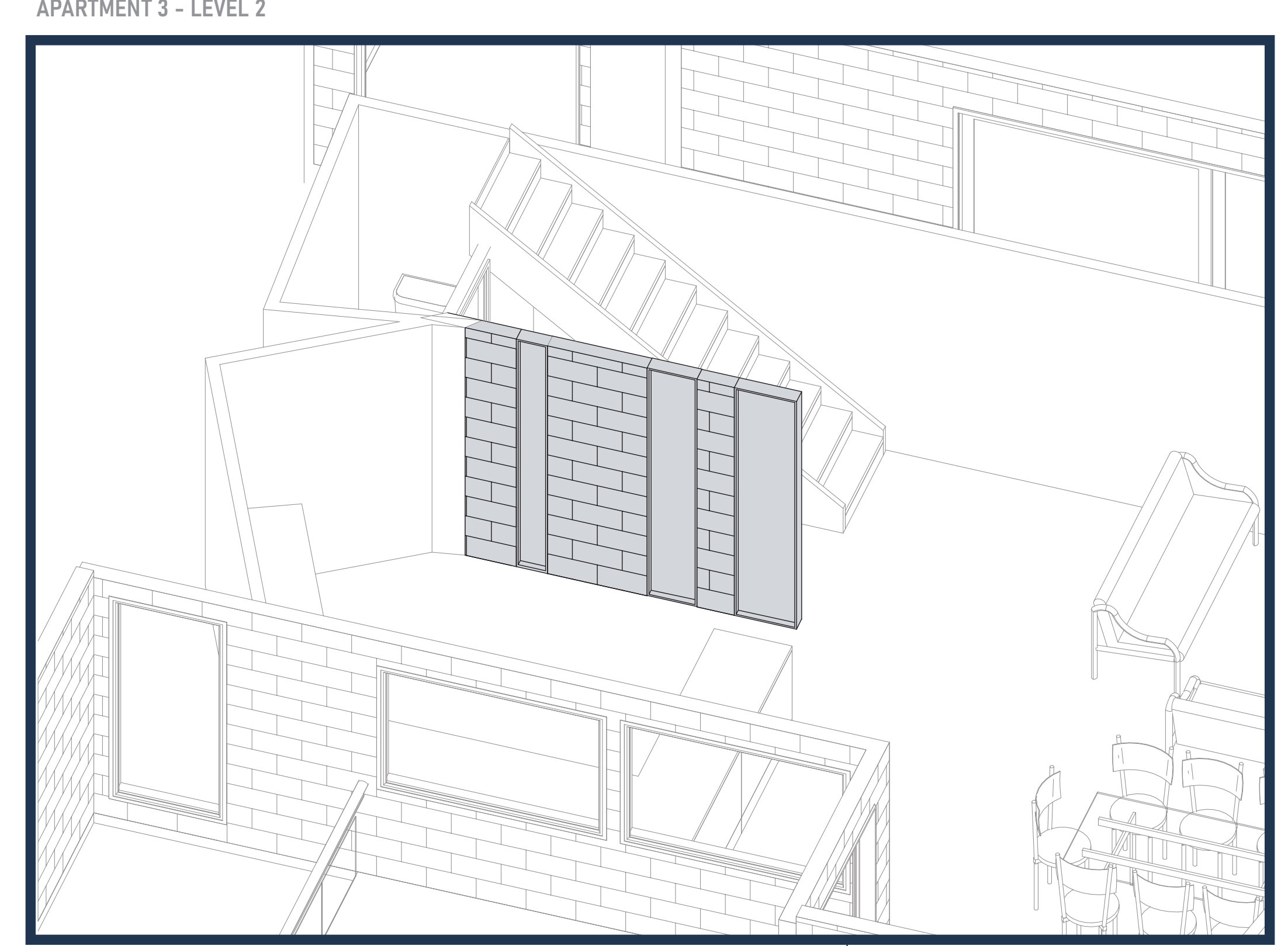

184 

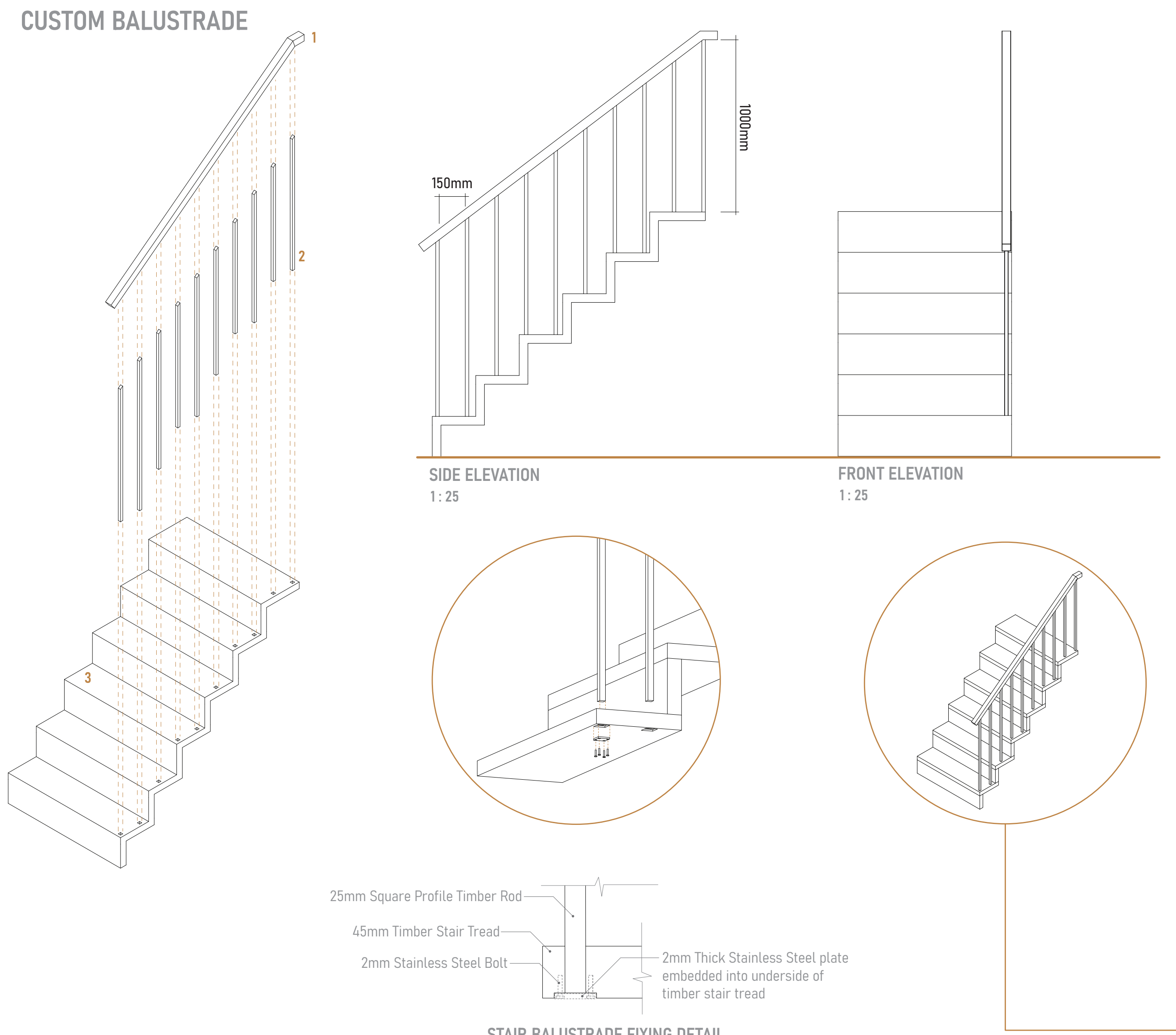

STAIR BALUSTRADE FIXING DETAIL 


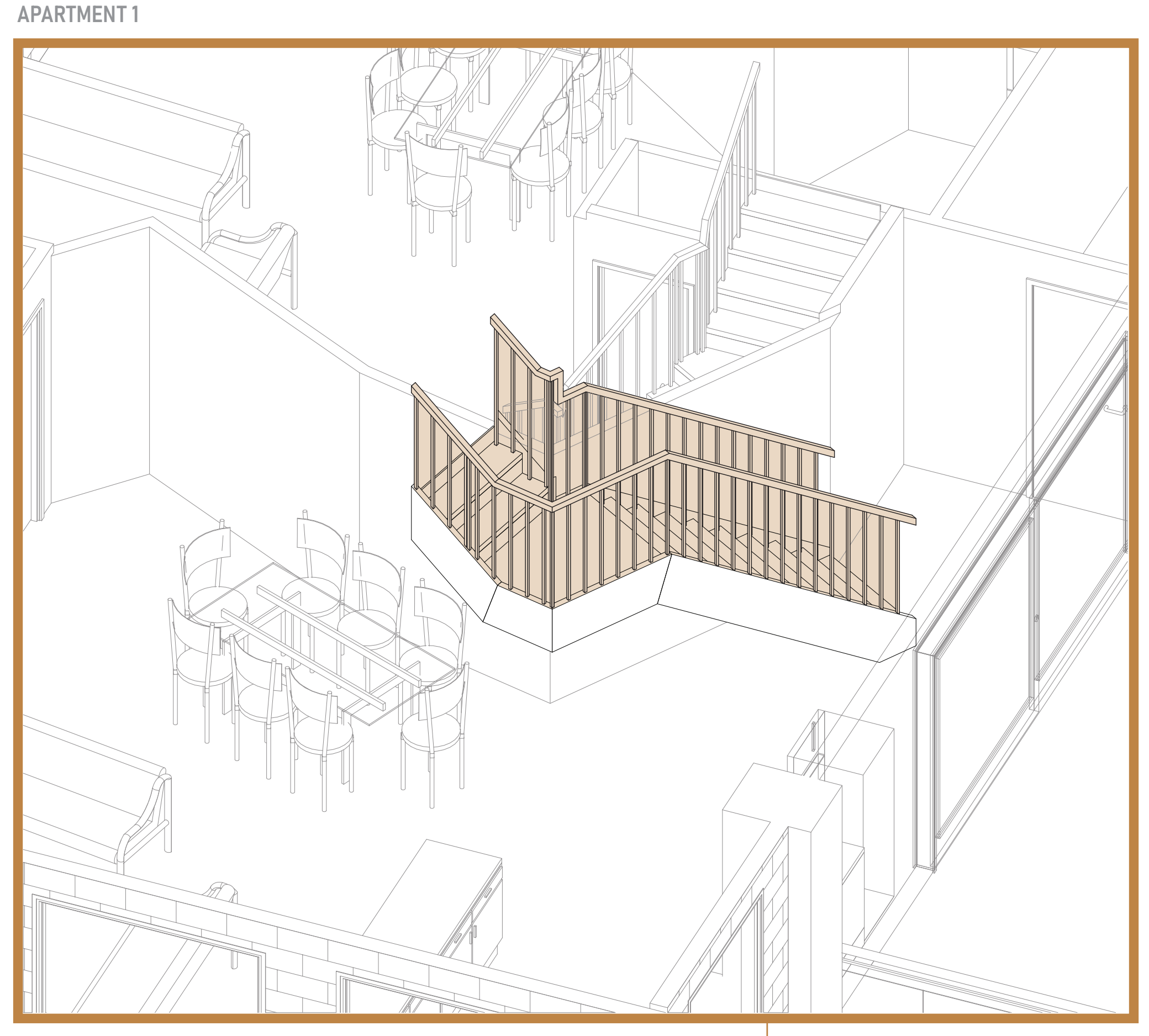

186 
CUSTOM KITCHEN CABINETRY

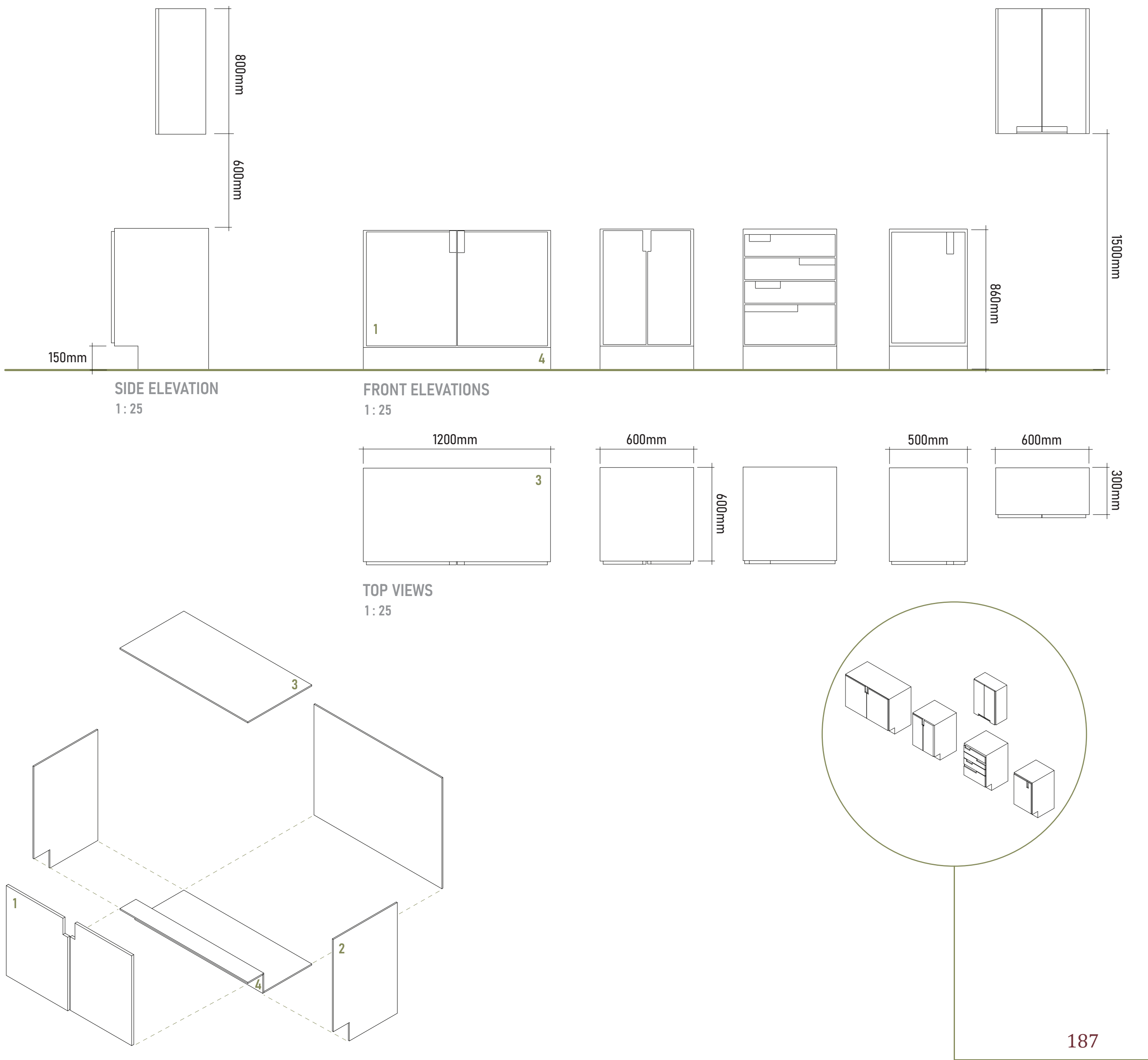




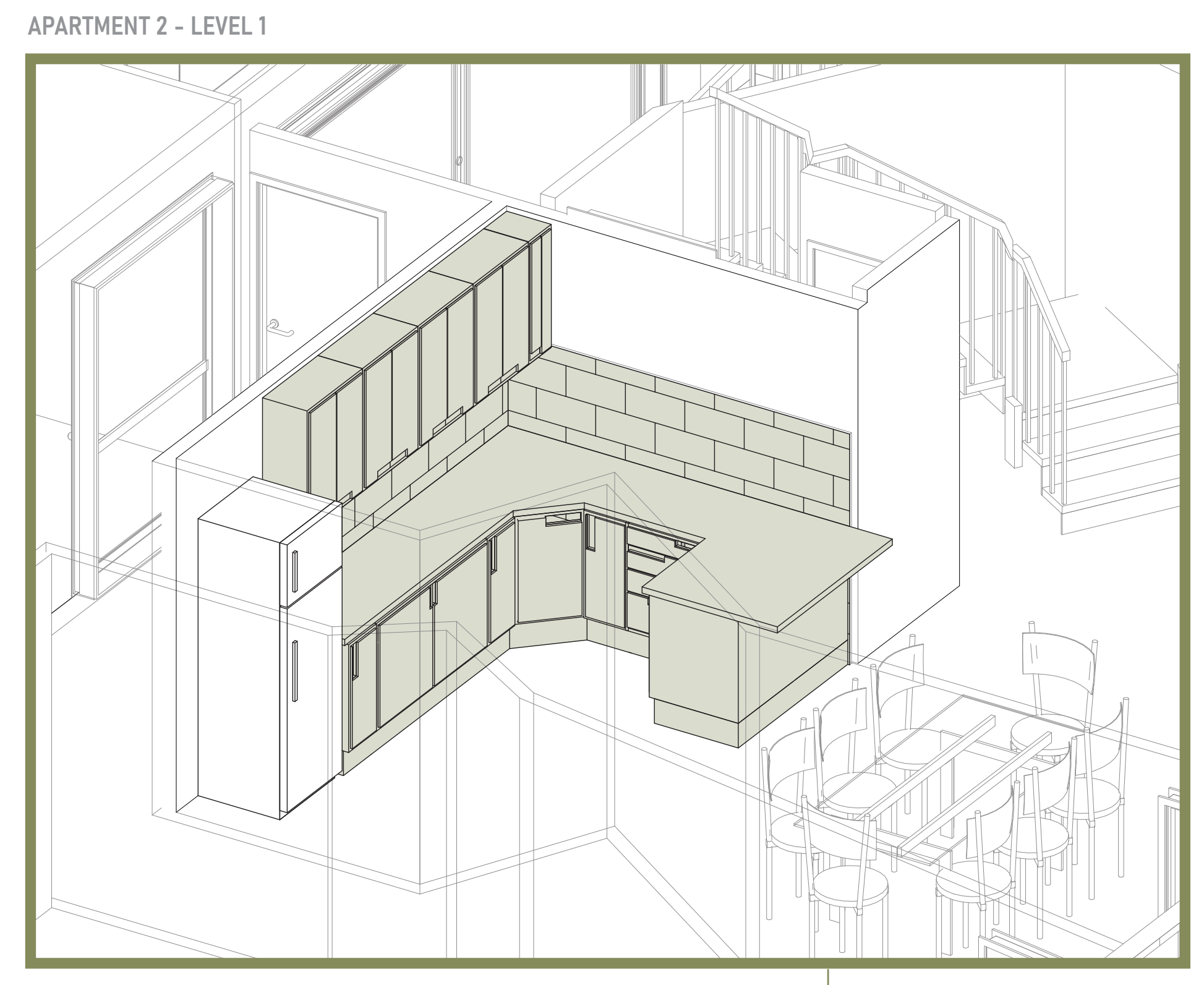

188 


\section{FINAL FLOOR PLAN}

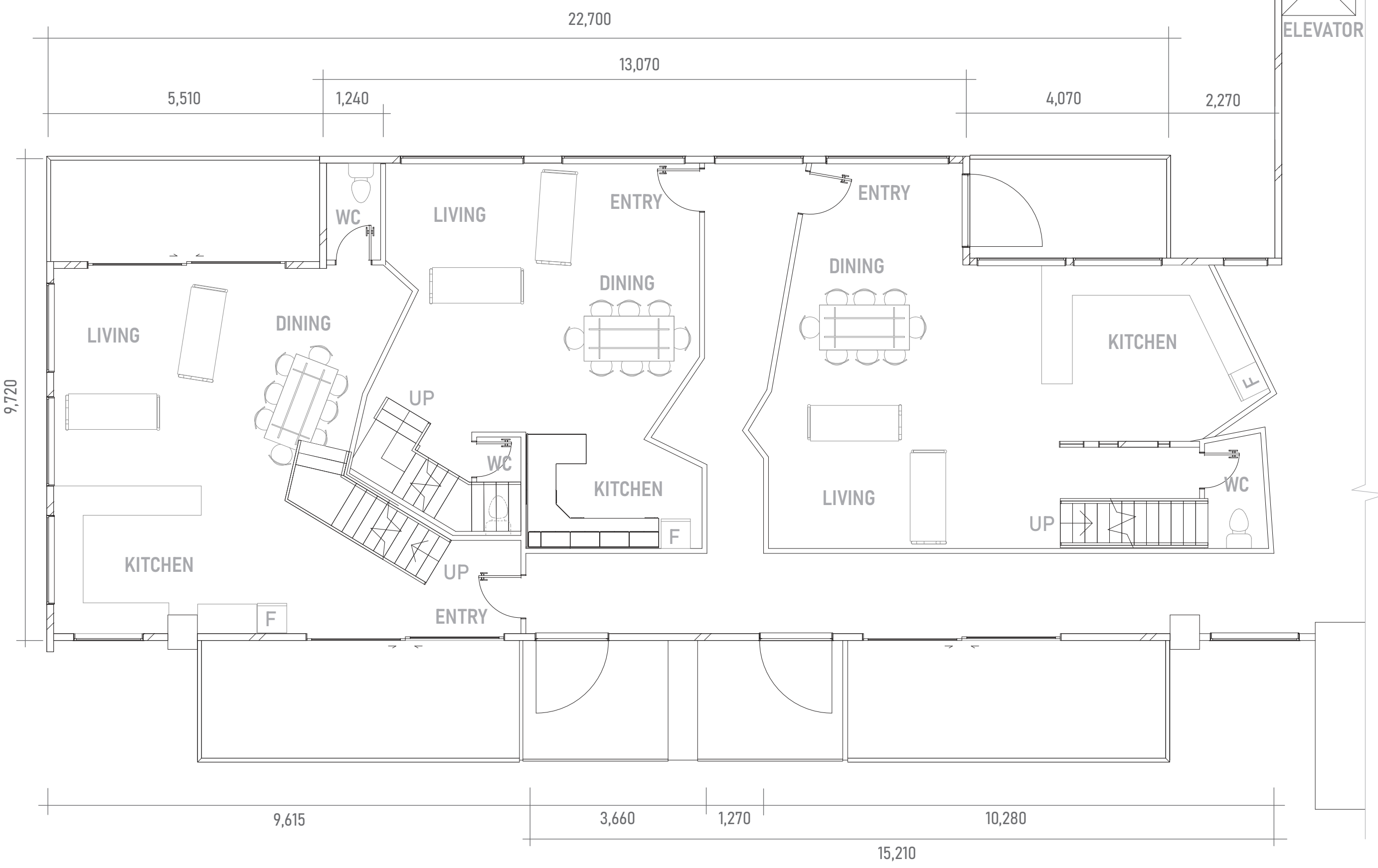

APARTMENTS - LEVEL 1

$1: 100$ 


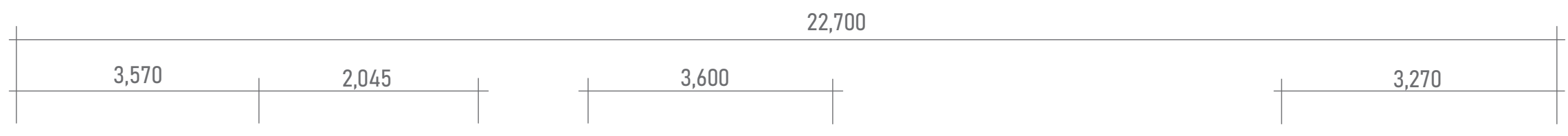

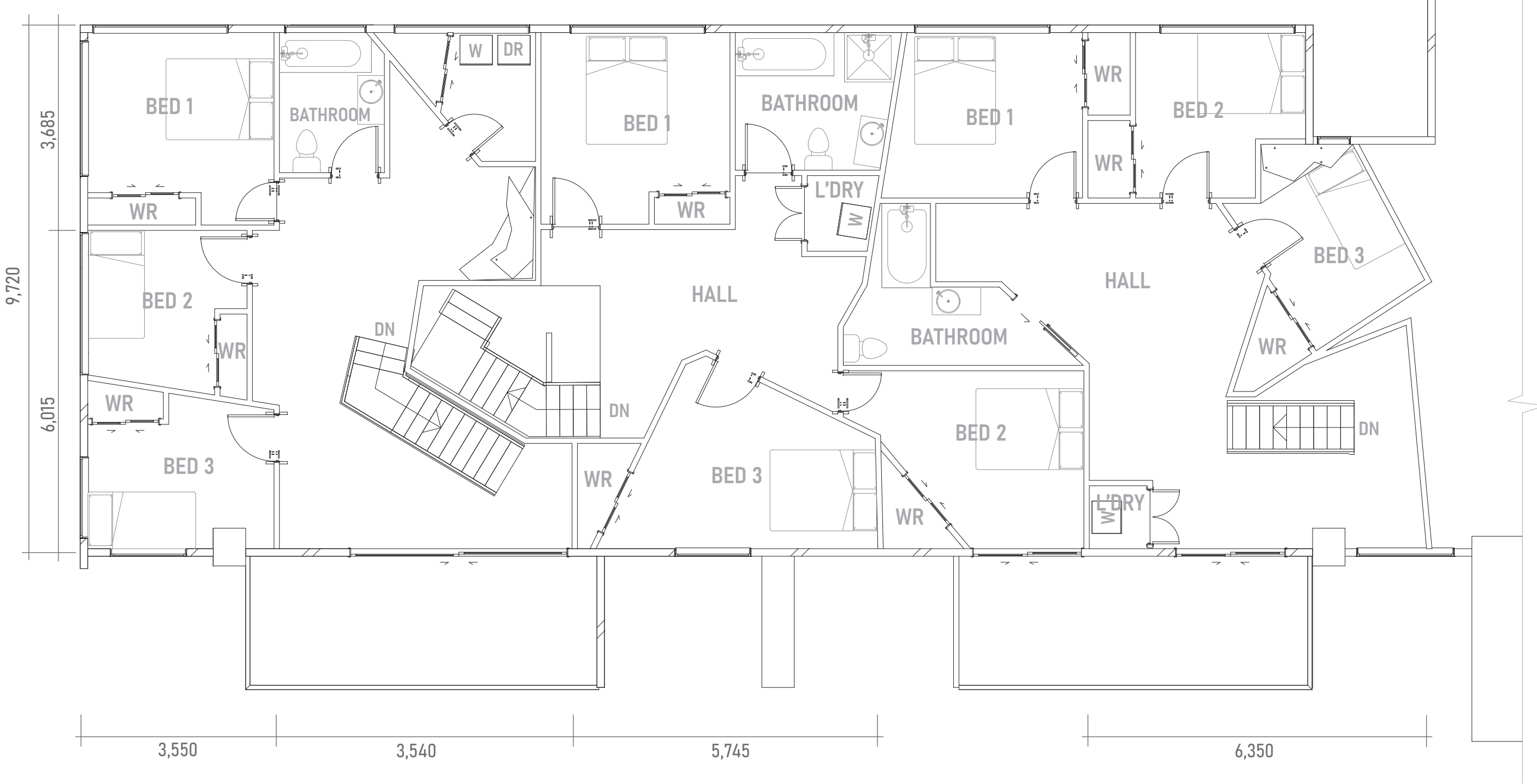

\section{APARTMENTS - LEVEL 2}

$1: 100$ 


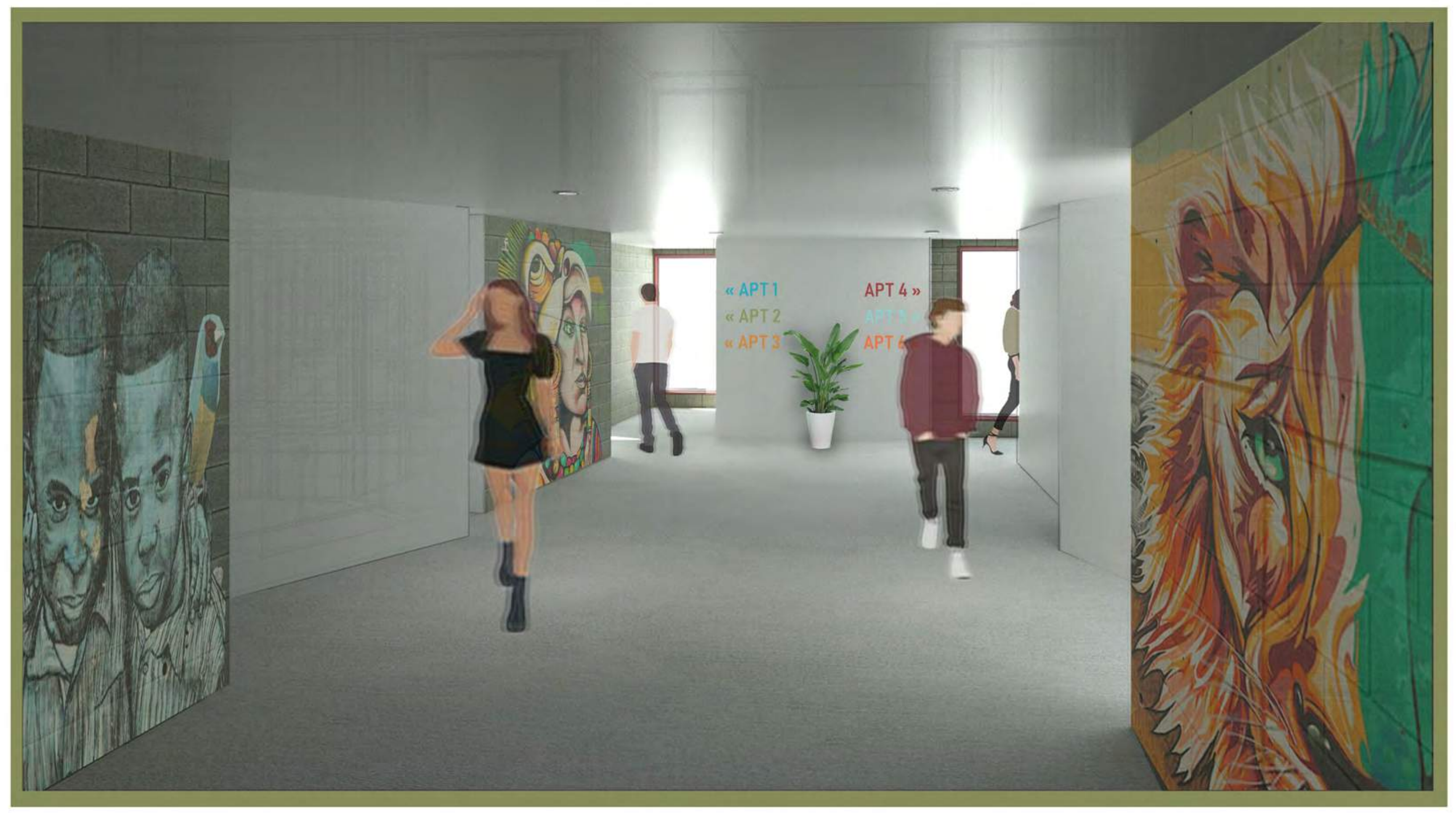

MAIN EXTERNAL HALLWAY 


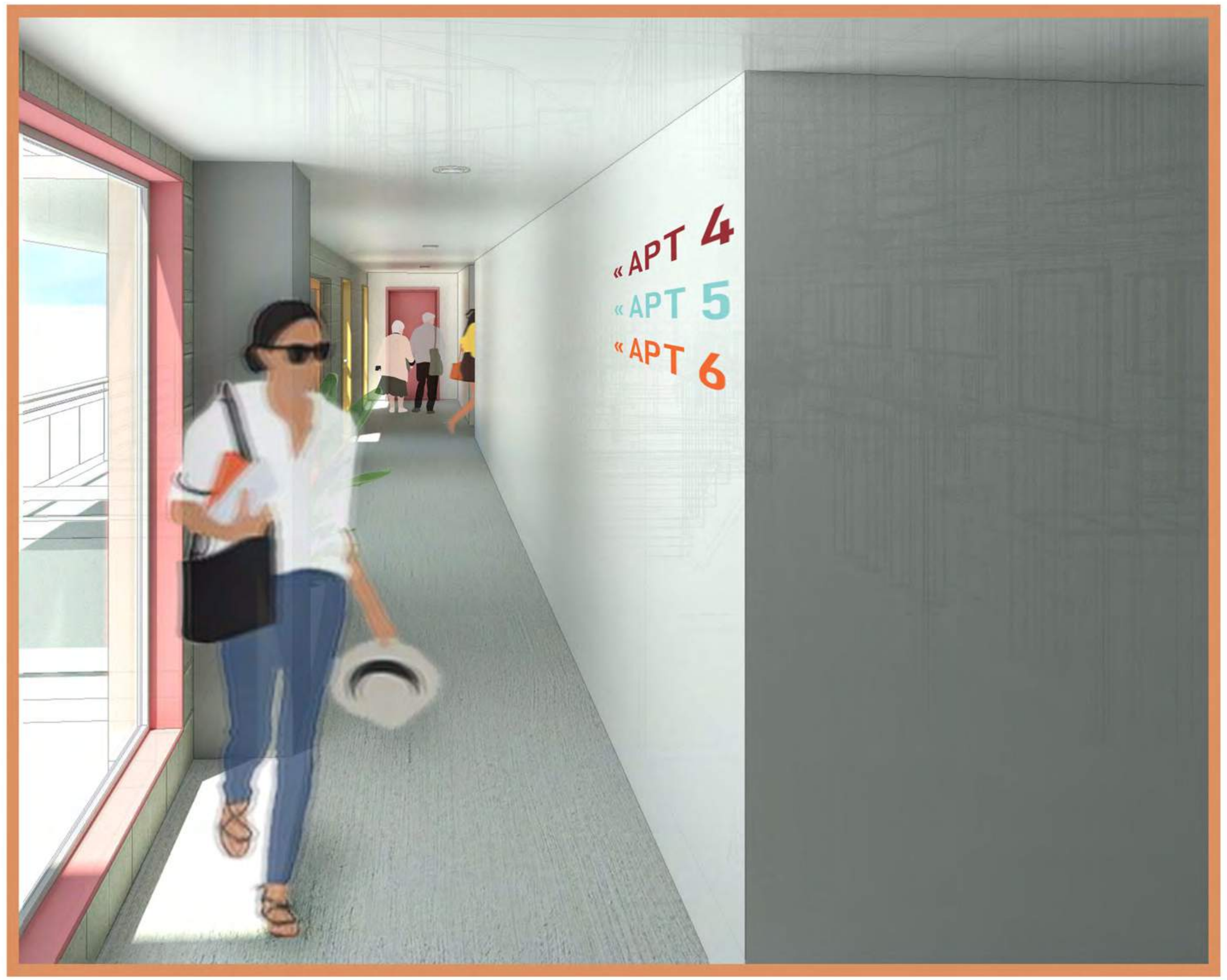

MAIN EXTERNAL HALLWAY 

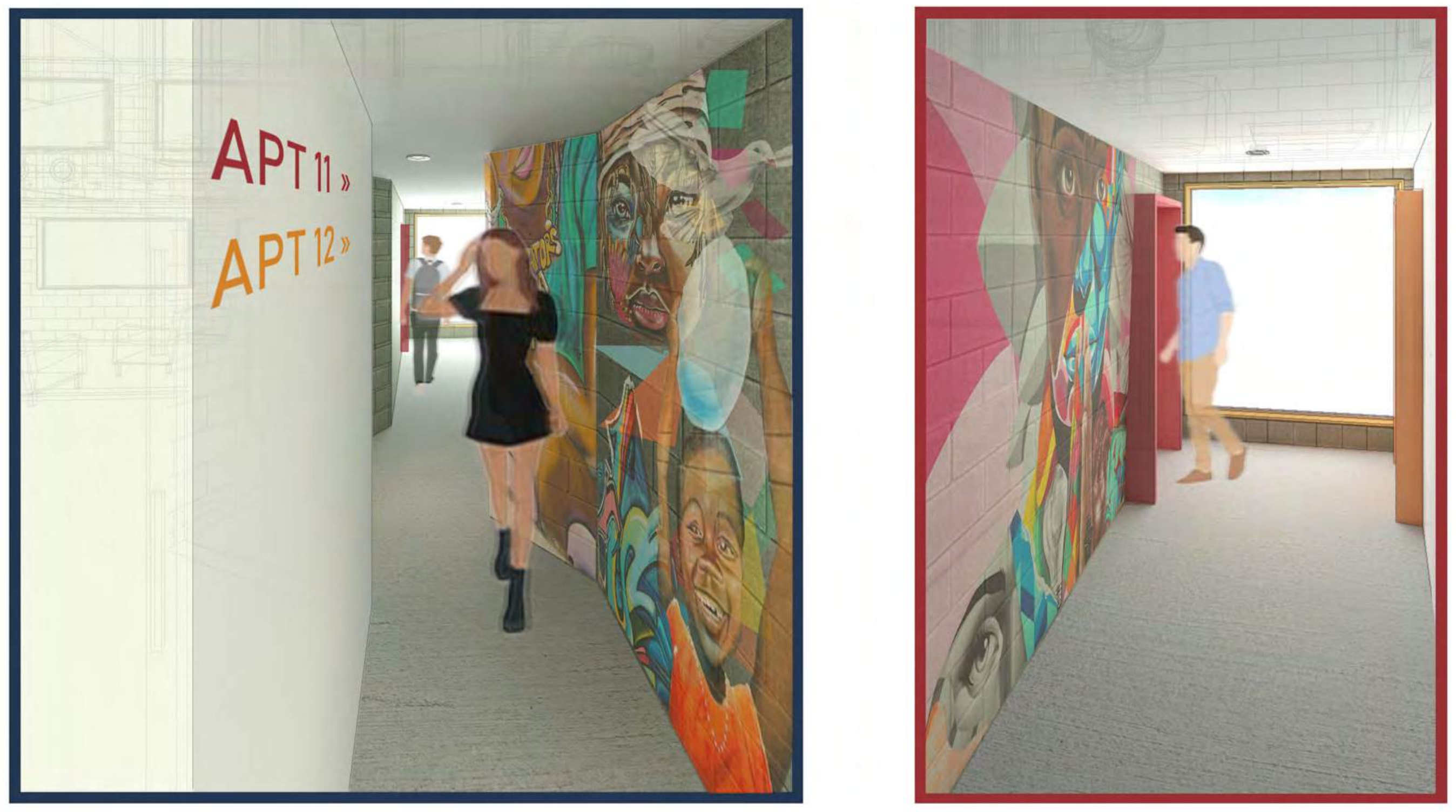

EXTERNAL HALLWAY 


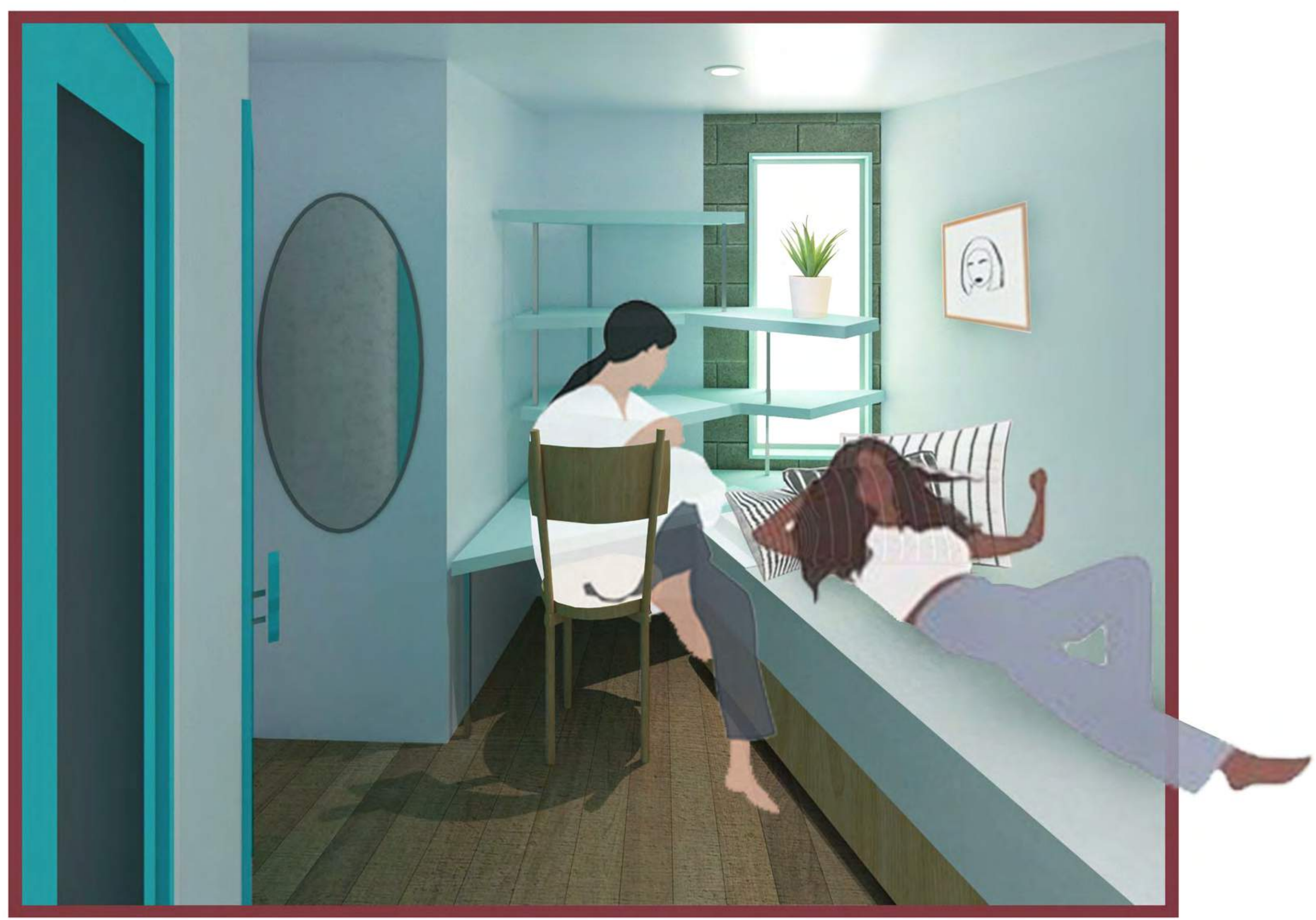

BEDROOM WITH CUSTOM DESK 


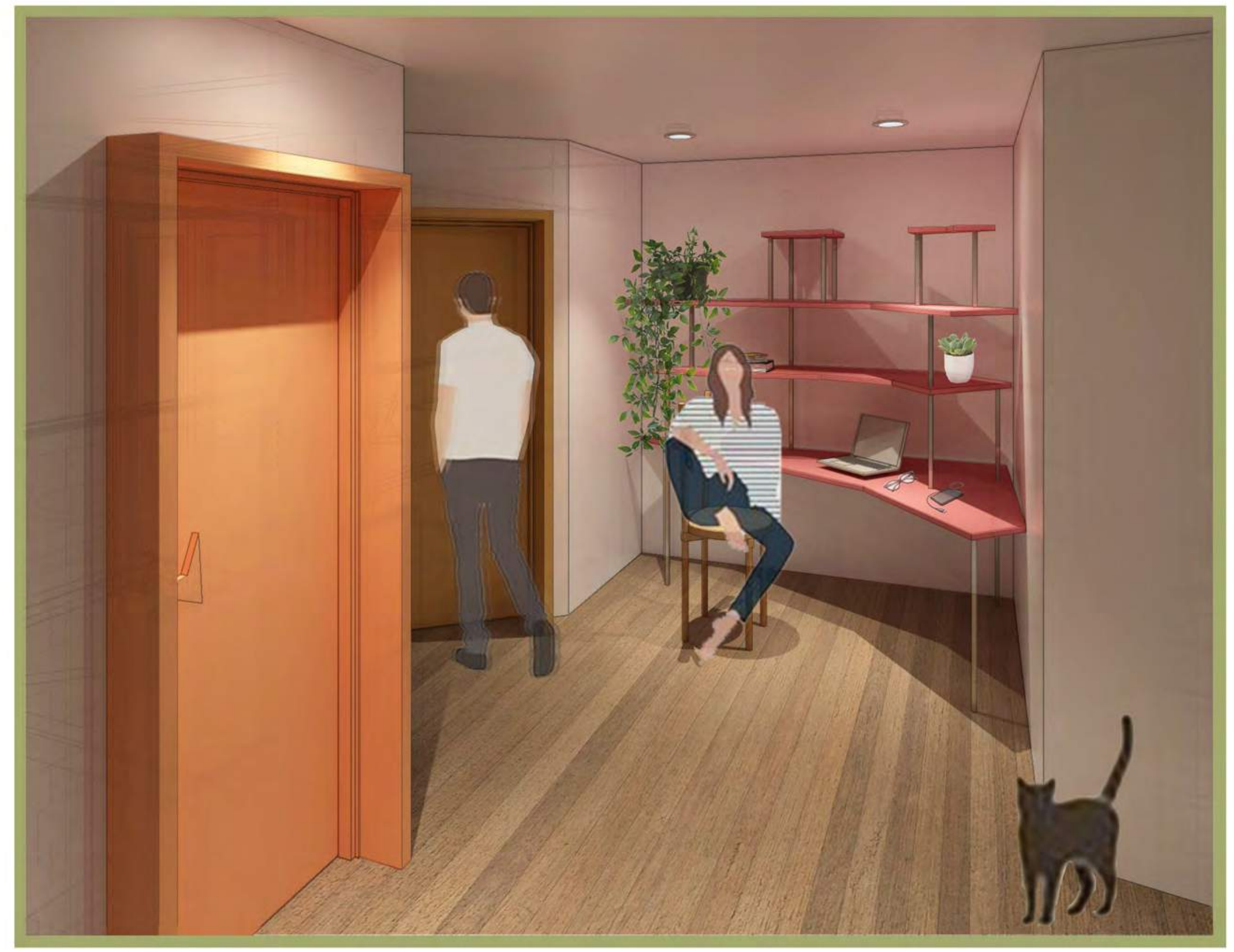

WORKSPACE 


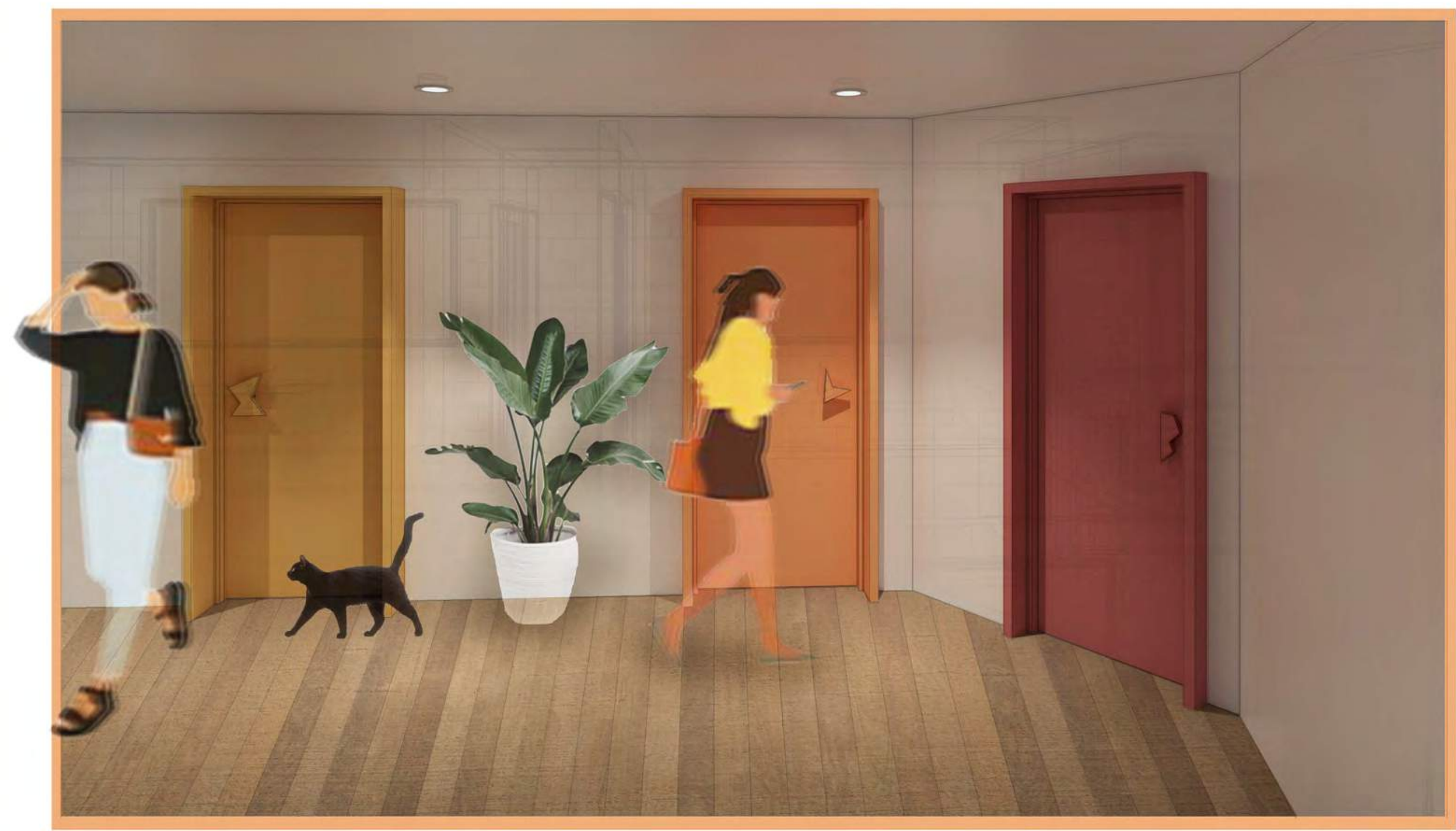

INTERNAL HALLWAY WITH CUSTOM DOORS 


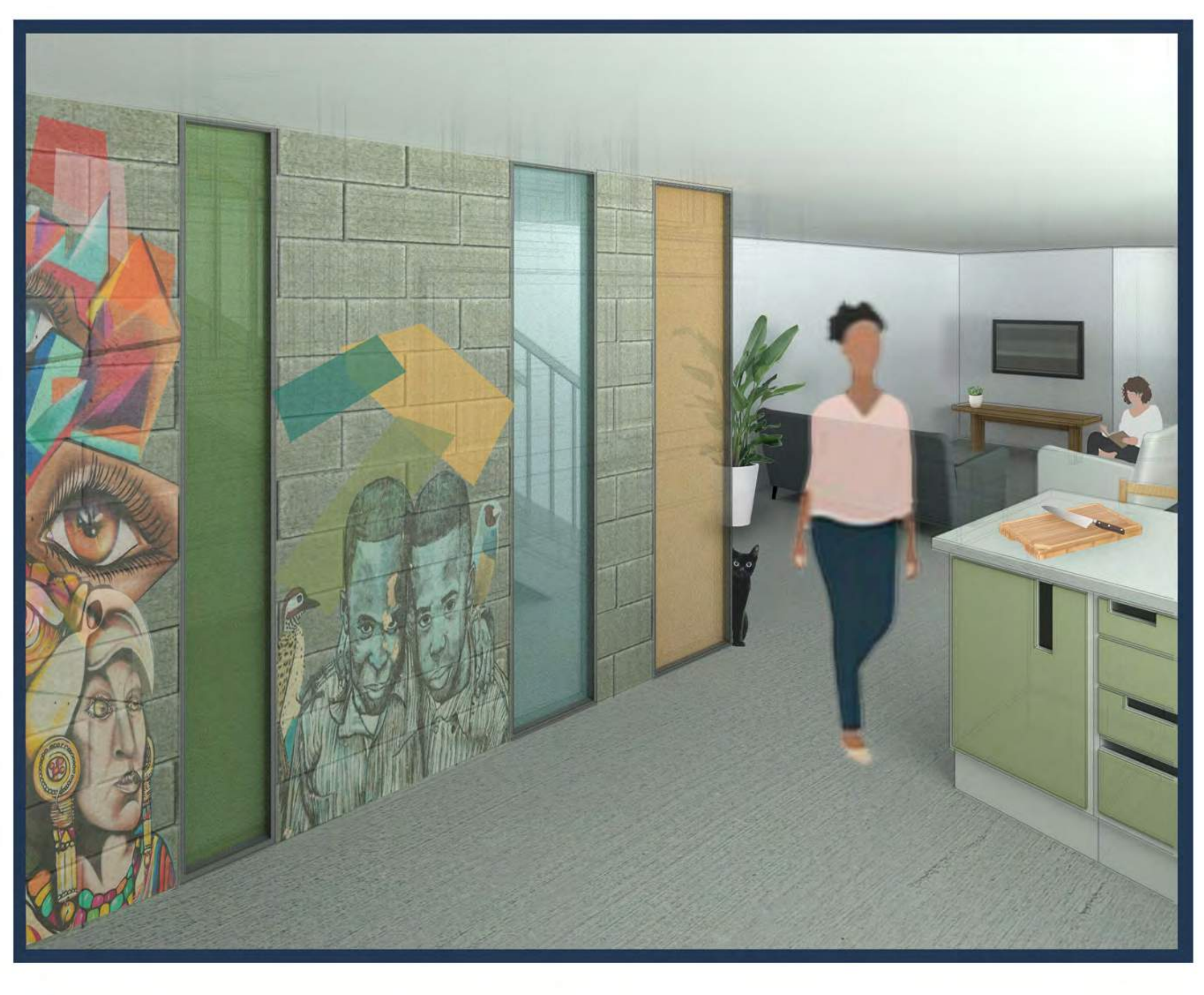




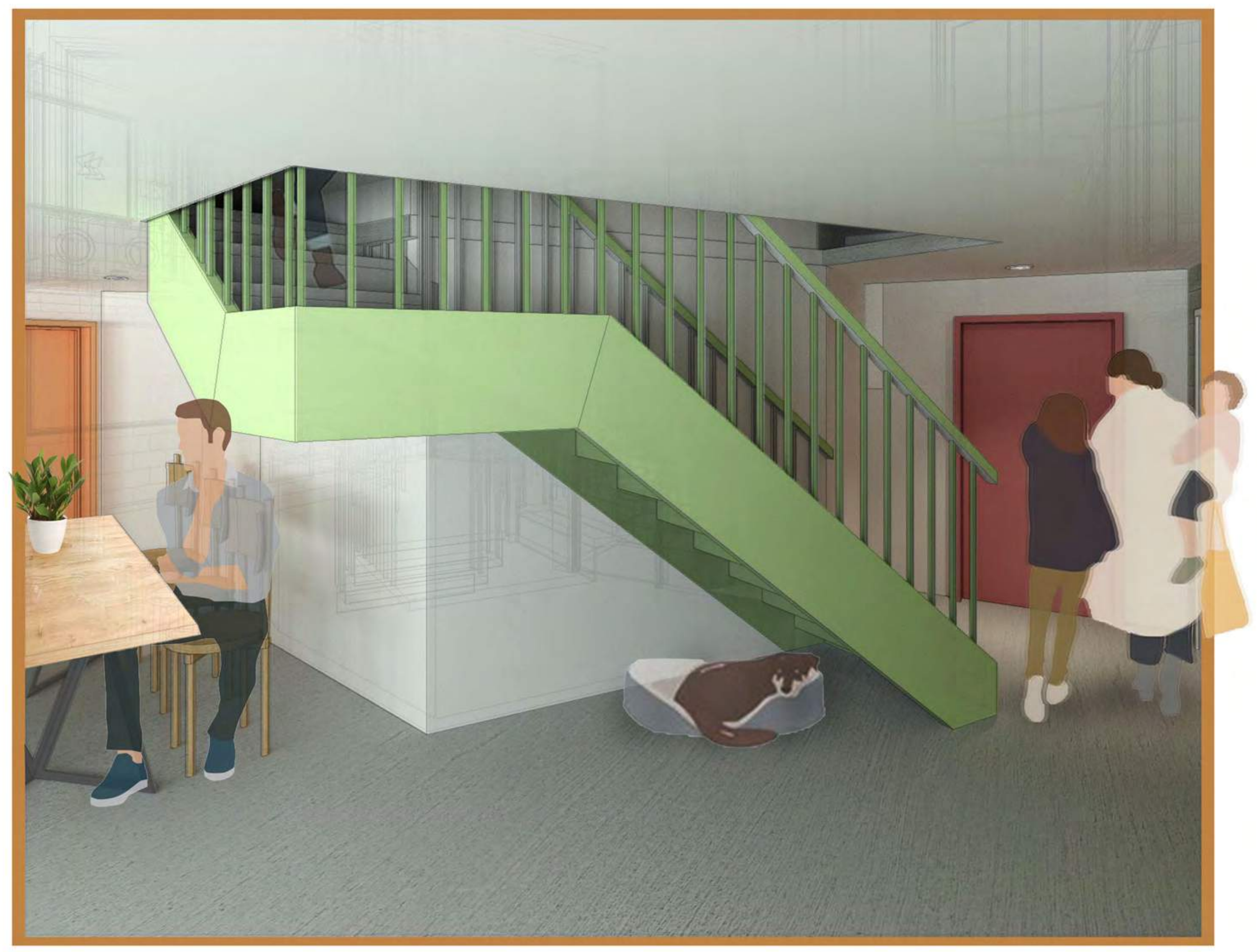

BALUSTRADE 


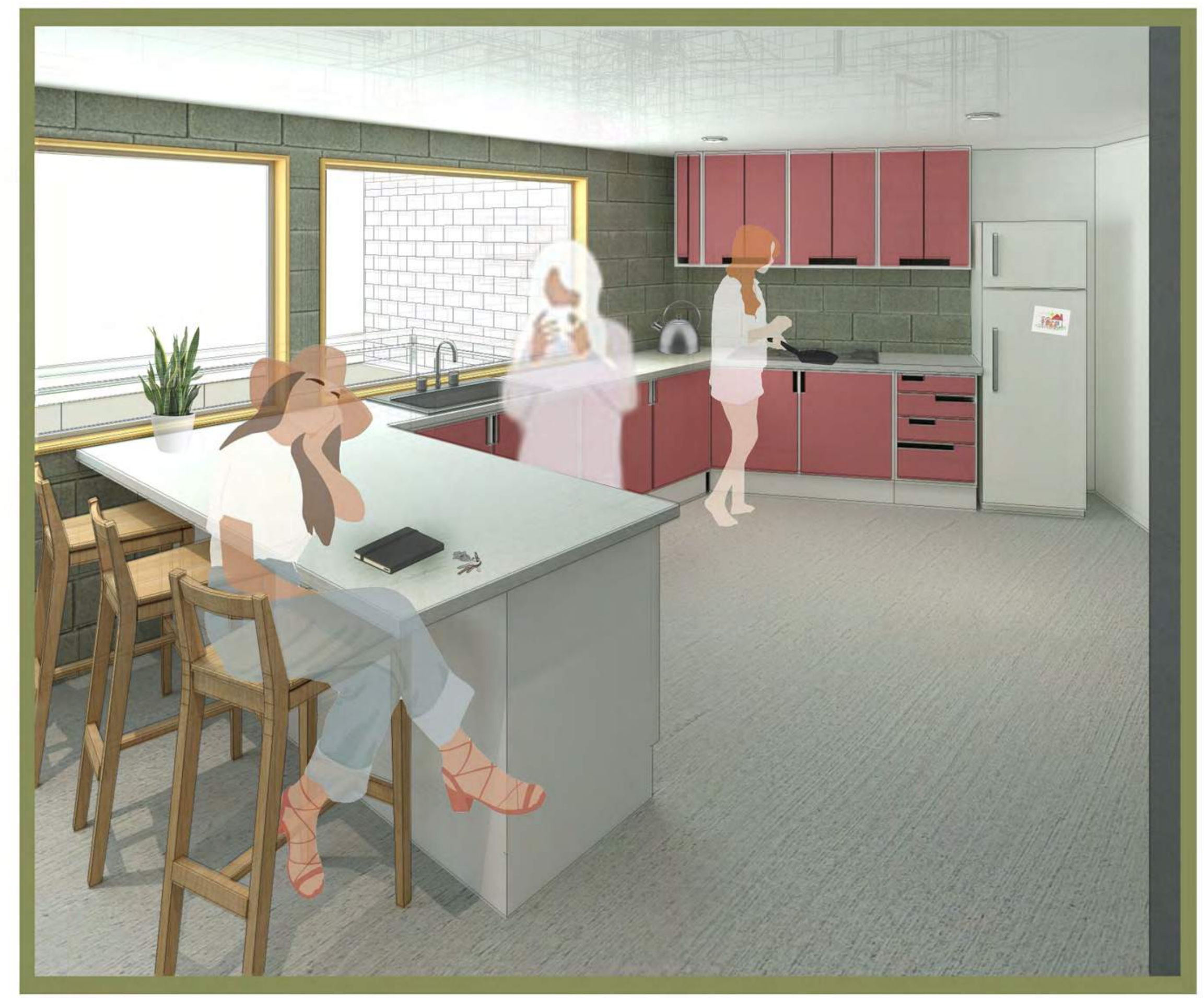

KITCHEN 


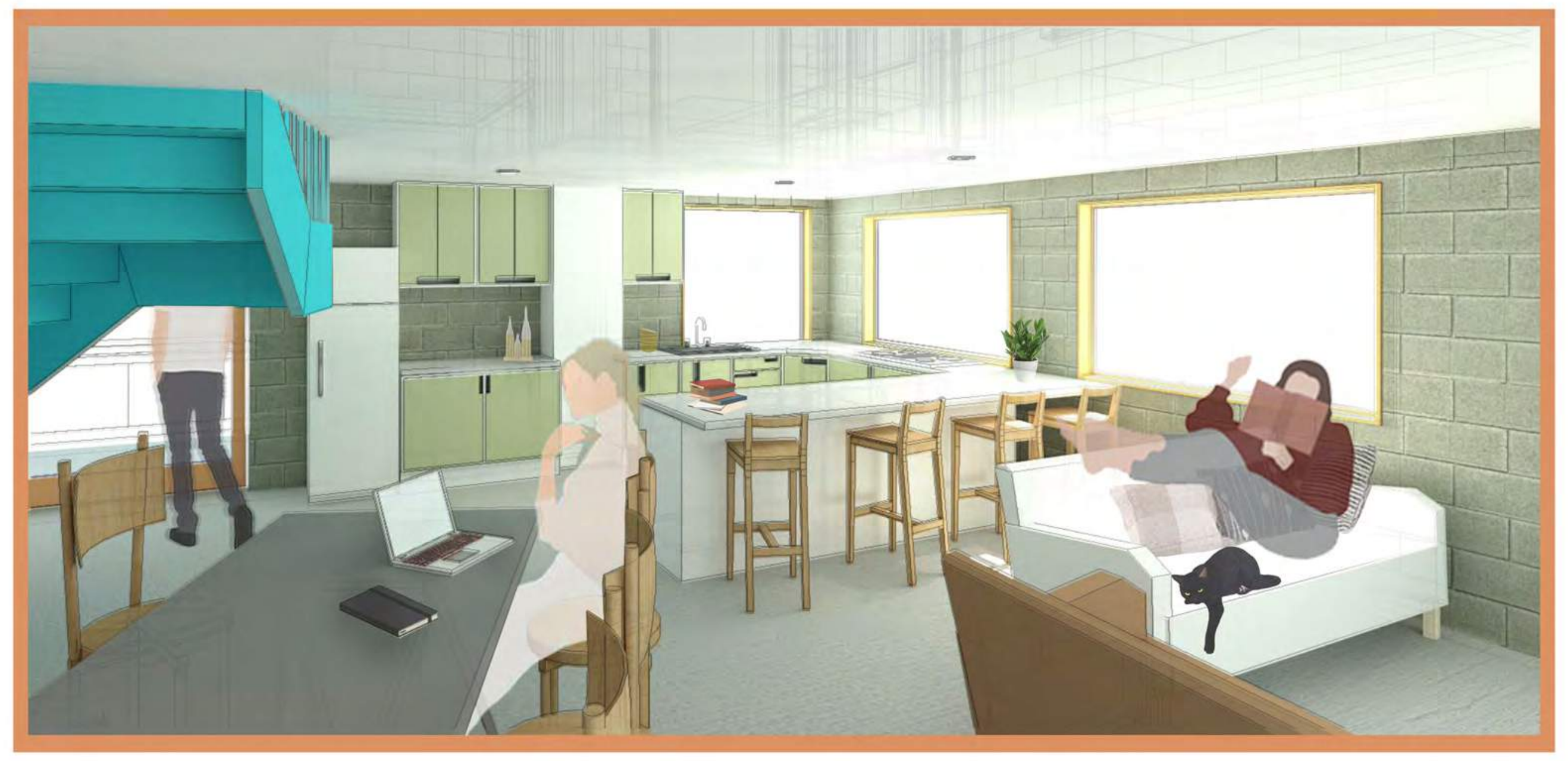

APARTMENT ONE 


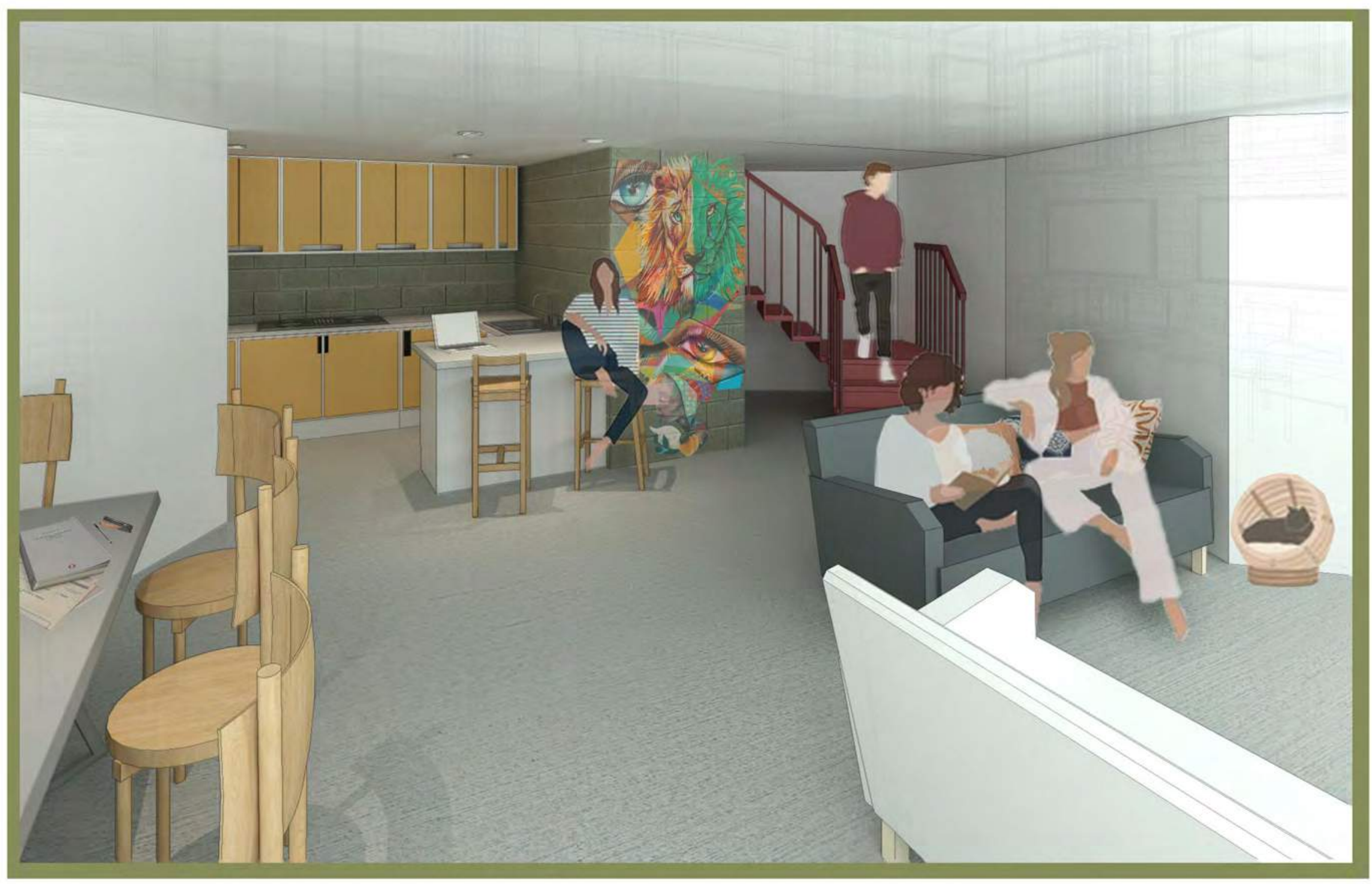

APARTMENT TWO 


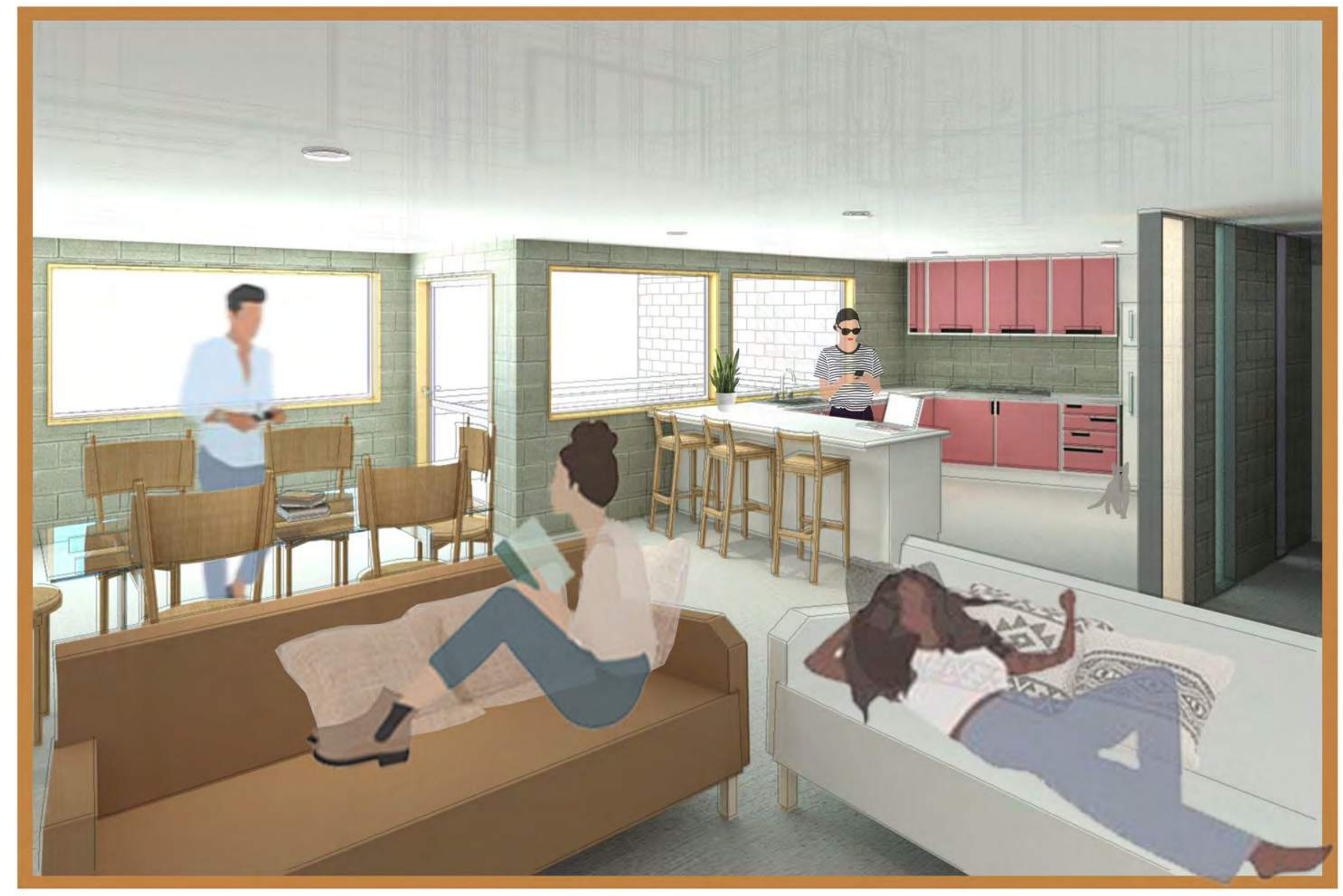

APARTMENT THREE 
2. COMMUNITY CENTRE

CENTRO COMUNITARIO 


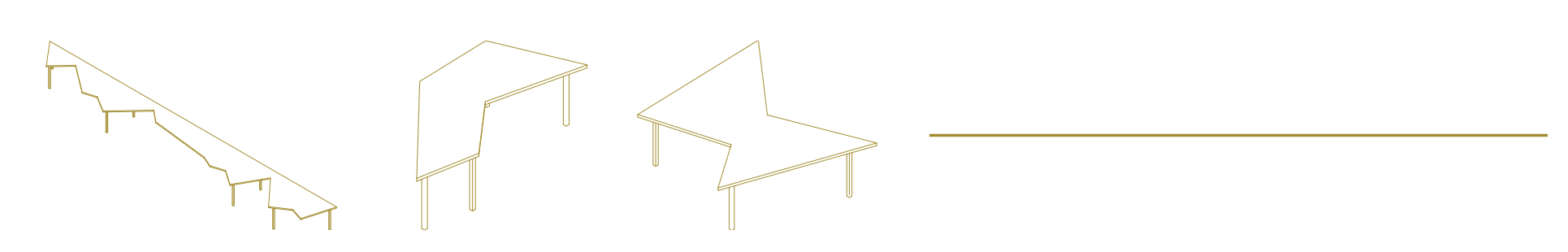


Throughout this research, it becomes evident that collaboration and coexistence are significant factors in this community's identity. Therefore, it is important for the design of their new living space to include an area for the community as a whole.

This is where the design of a community centre starts. In the early stages, it is established that this space has some very specific needs to cater to this community such as a space for dance and a space for workshops and learning.

The aim is to give the community a space which felt truly theirs but also to give the youth of the community a space to be creative and hang out. A space which makes what they do feel important but also gives them a space in which they can continue to grow and develop their art as well as their strong sense of community. 
"Cuando hay que hacer y hay que juntarse, yo creo que La Comuna 13 abe hacerlo." - Koala

"When you have to do something and you have to get together, I believe that La Comuna 13 knows how to do it." 
DANCE STUDIO

To follow the same process as pavillion design with angled walls
NEEDS:

- Dancing space

- Seating area

- Toilets

- Circulation

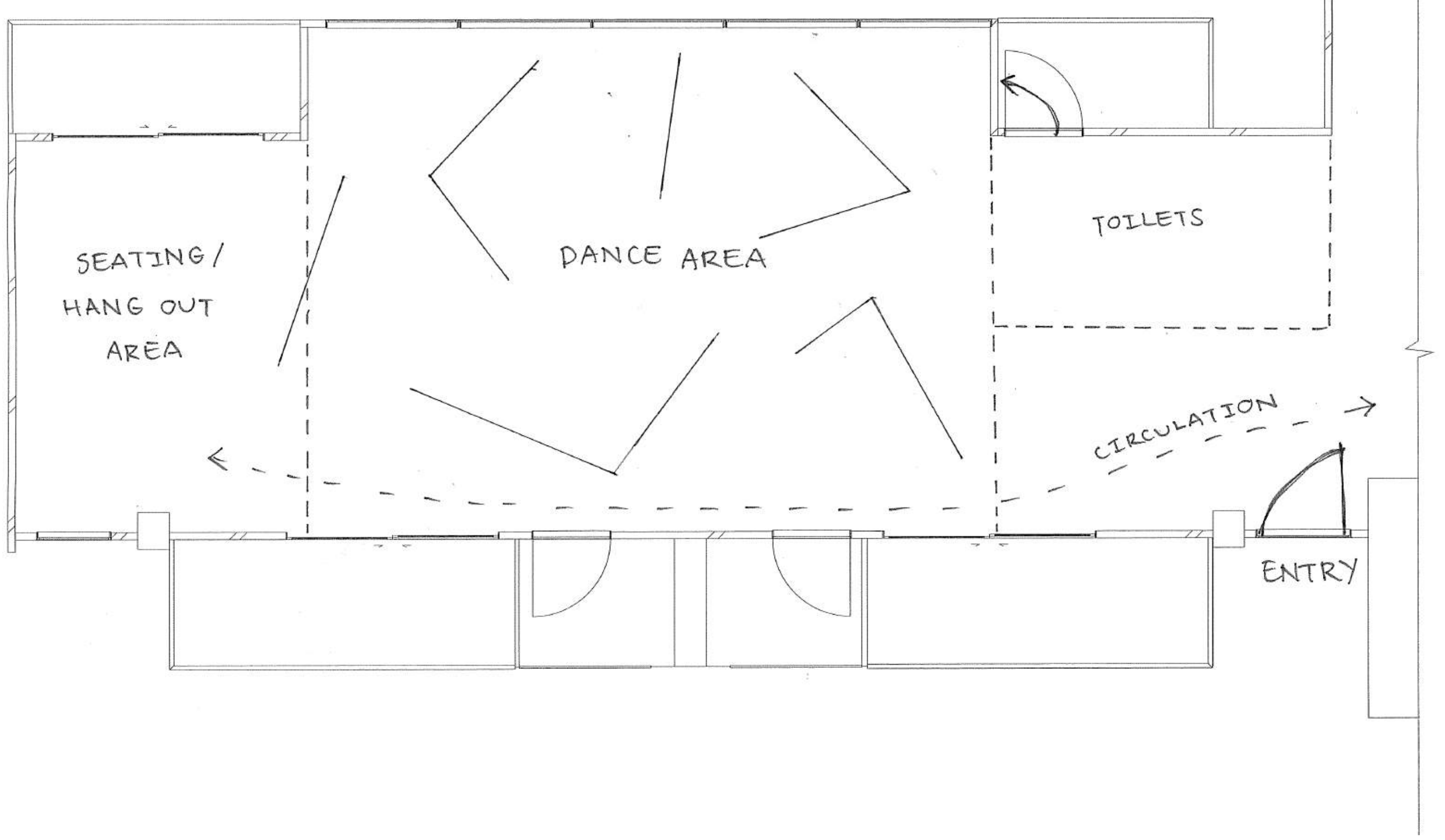

207 


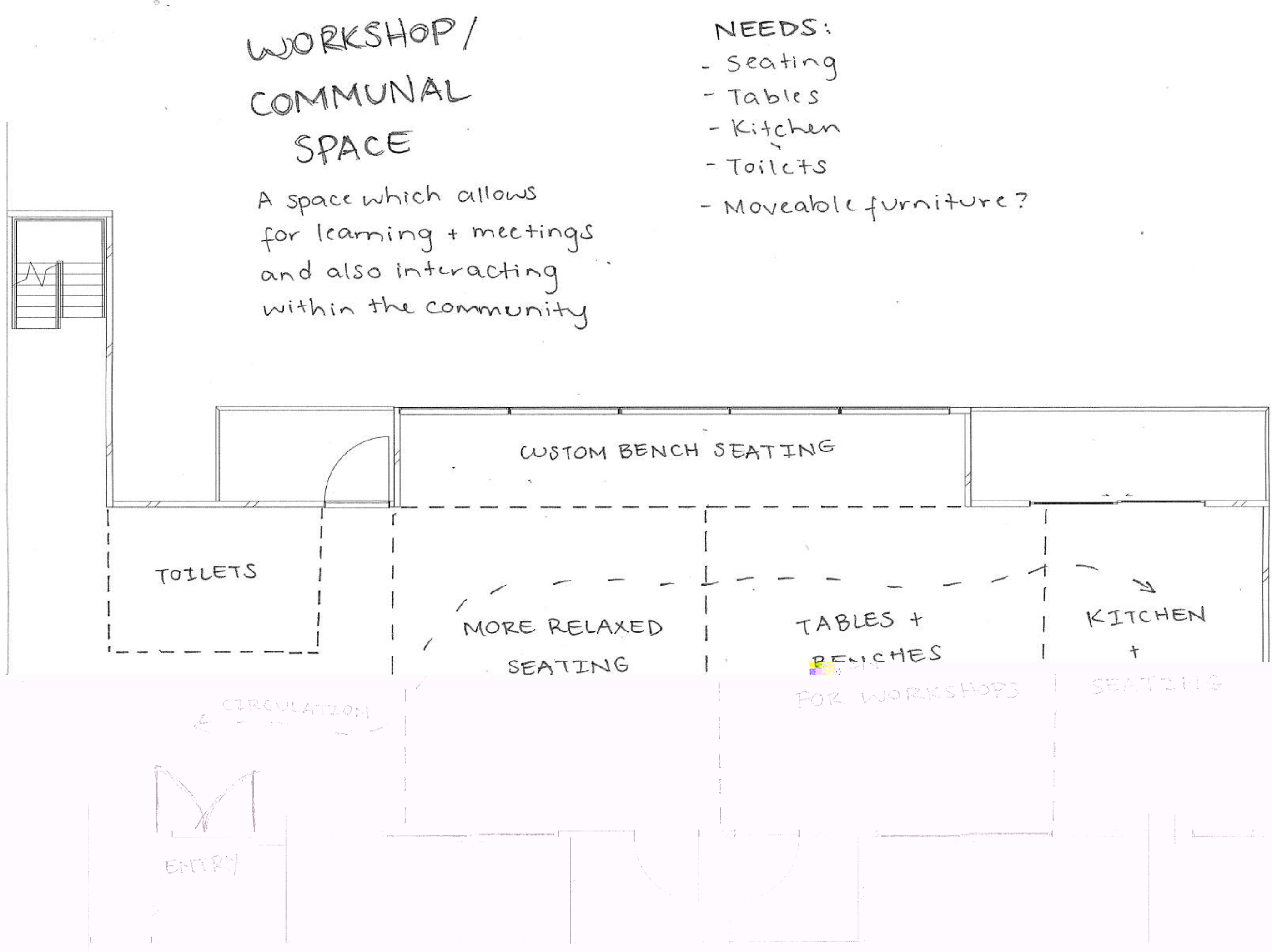

208 
THE DANCE AREA

PHASE 1: THE SHAPES

$13 m$

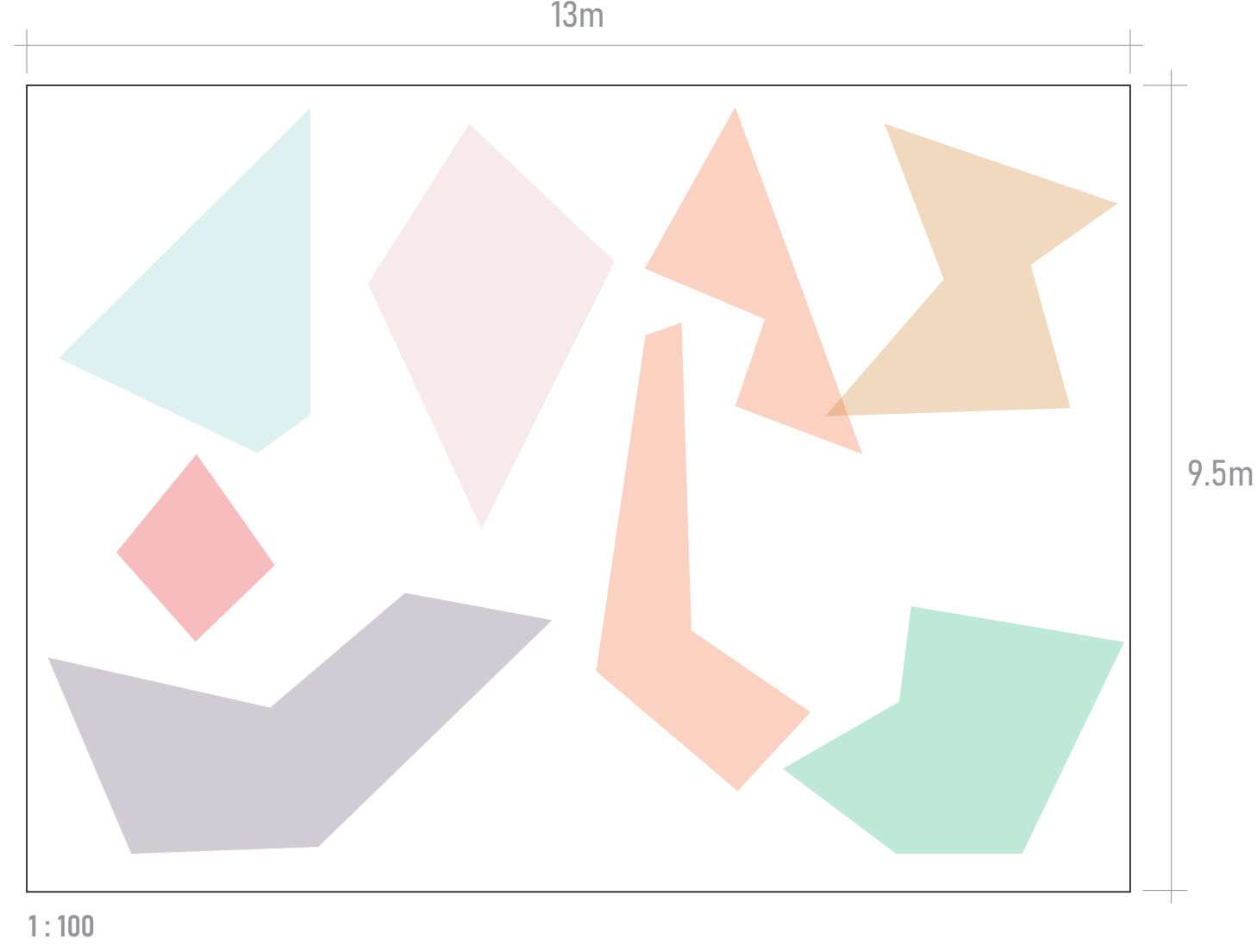

PHASE 3: THE WALLS

THE WALLS

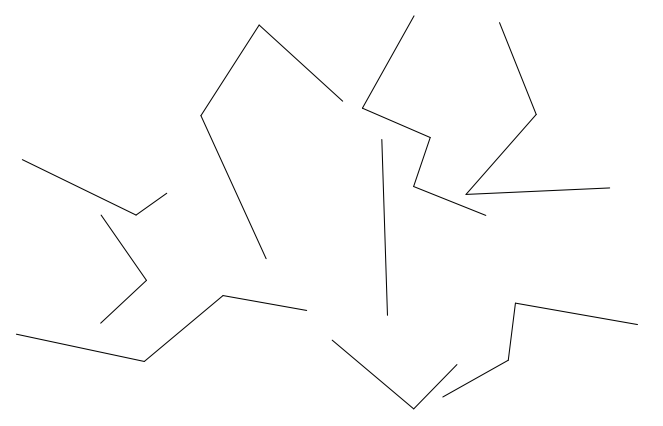

THE FLOOR PLAN

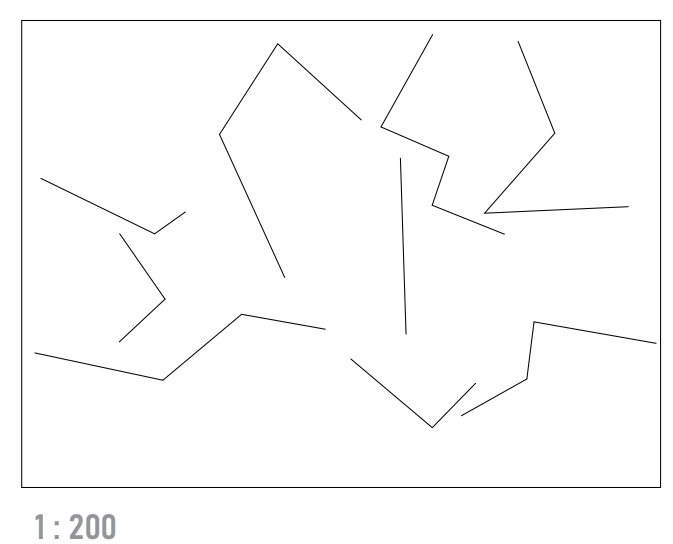

PHASE 2: THE EXTRUSION
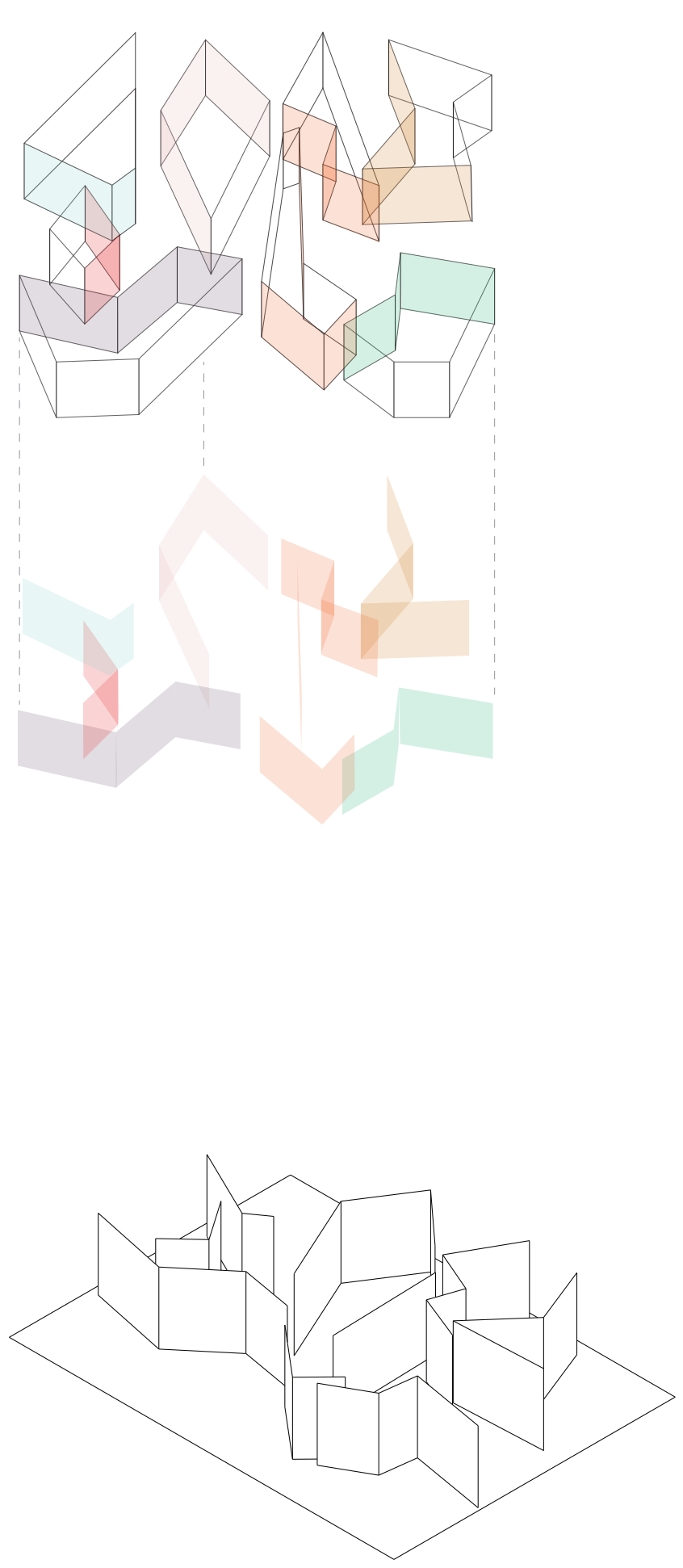


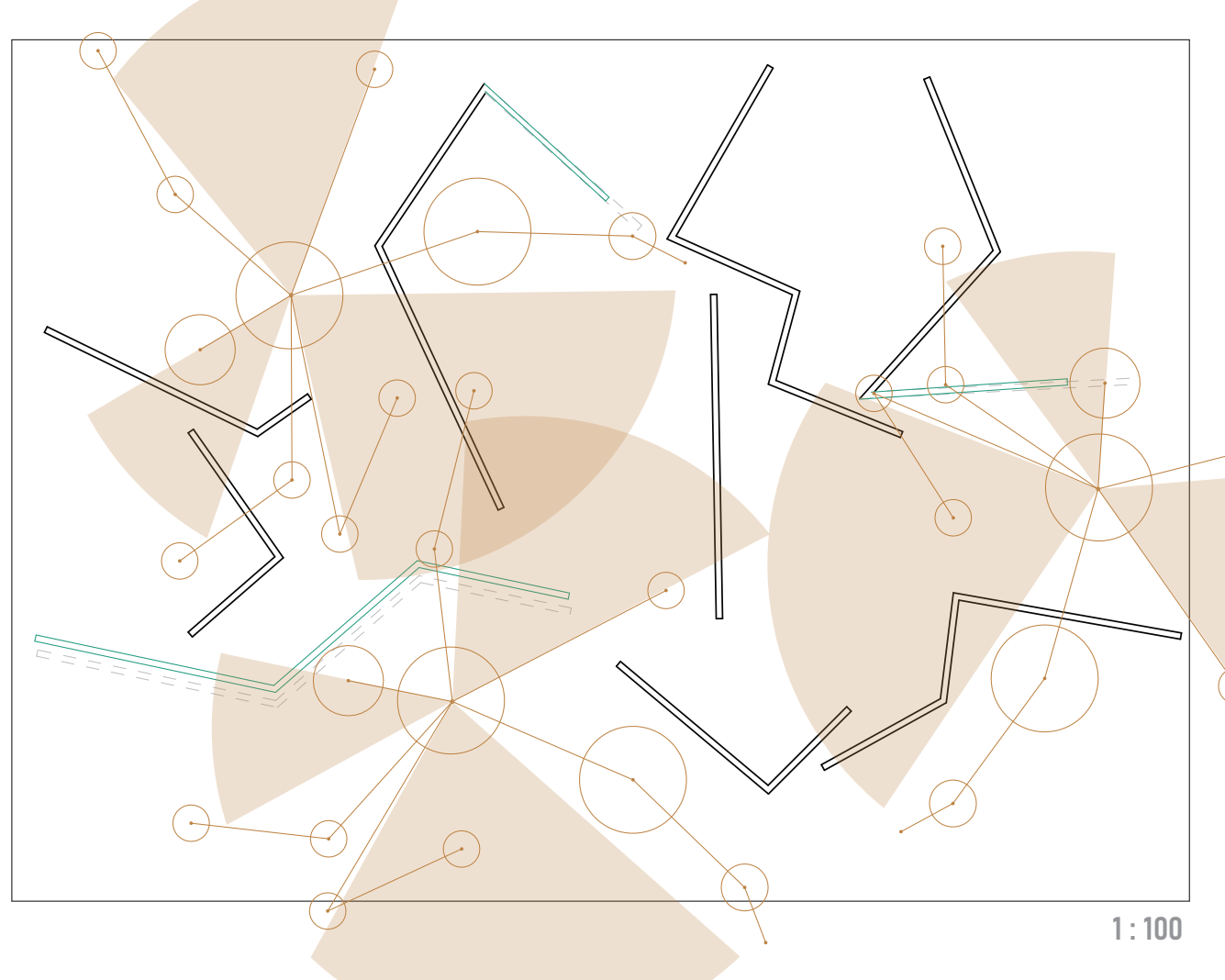

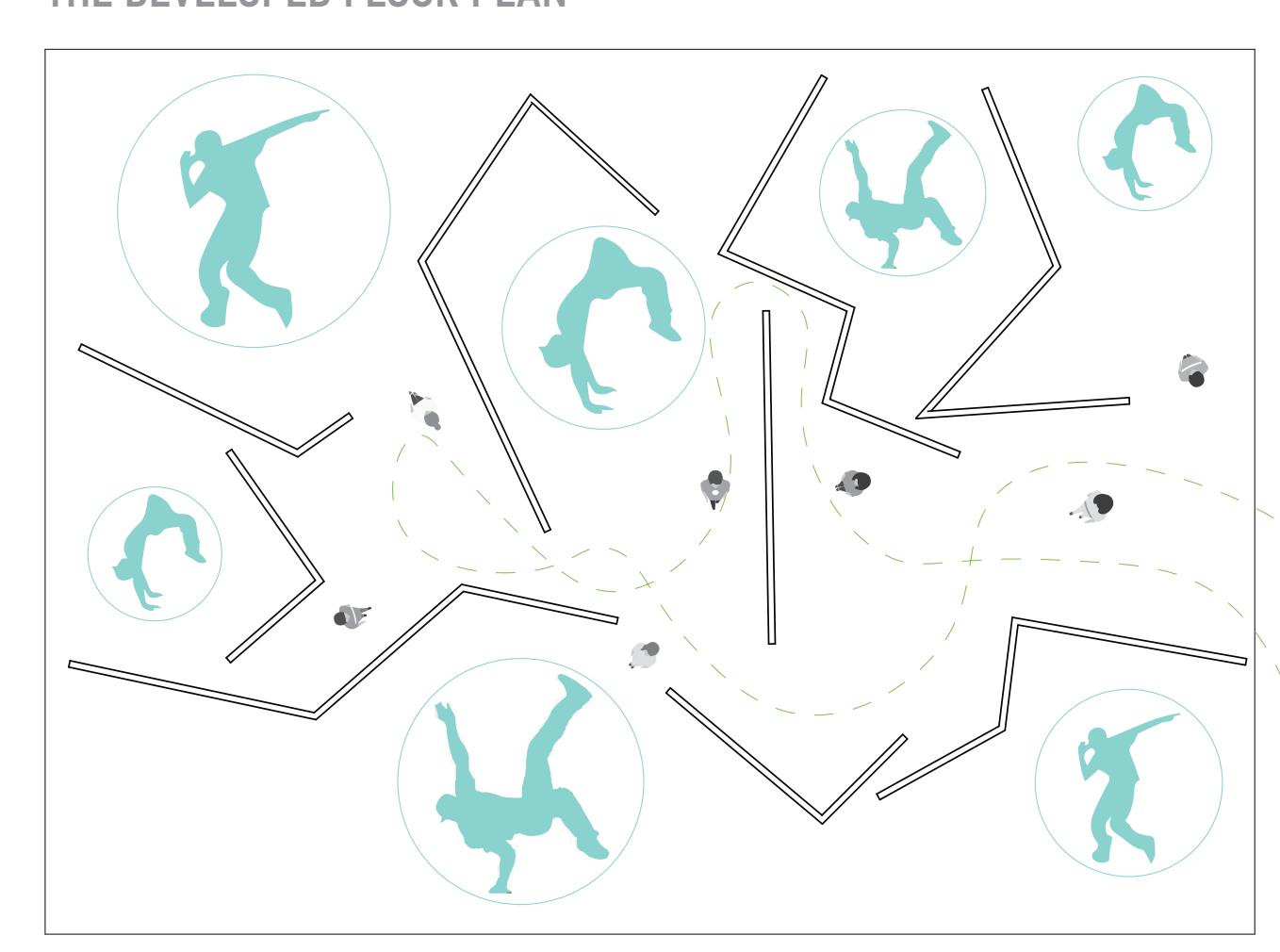




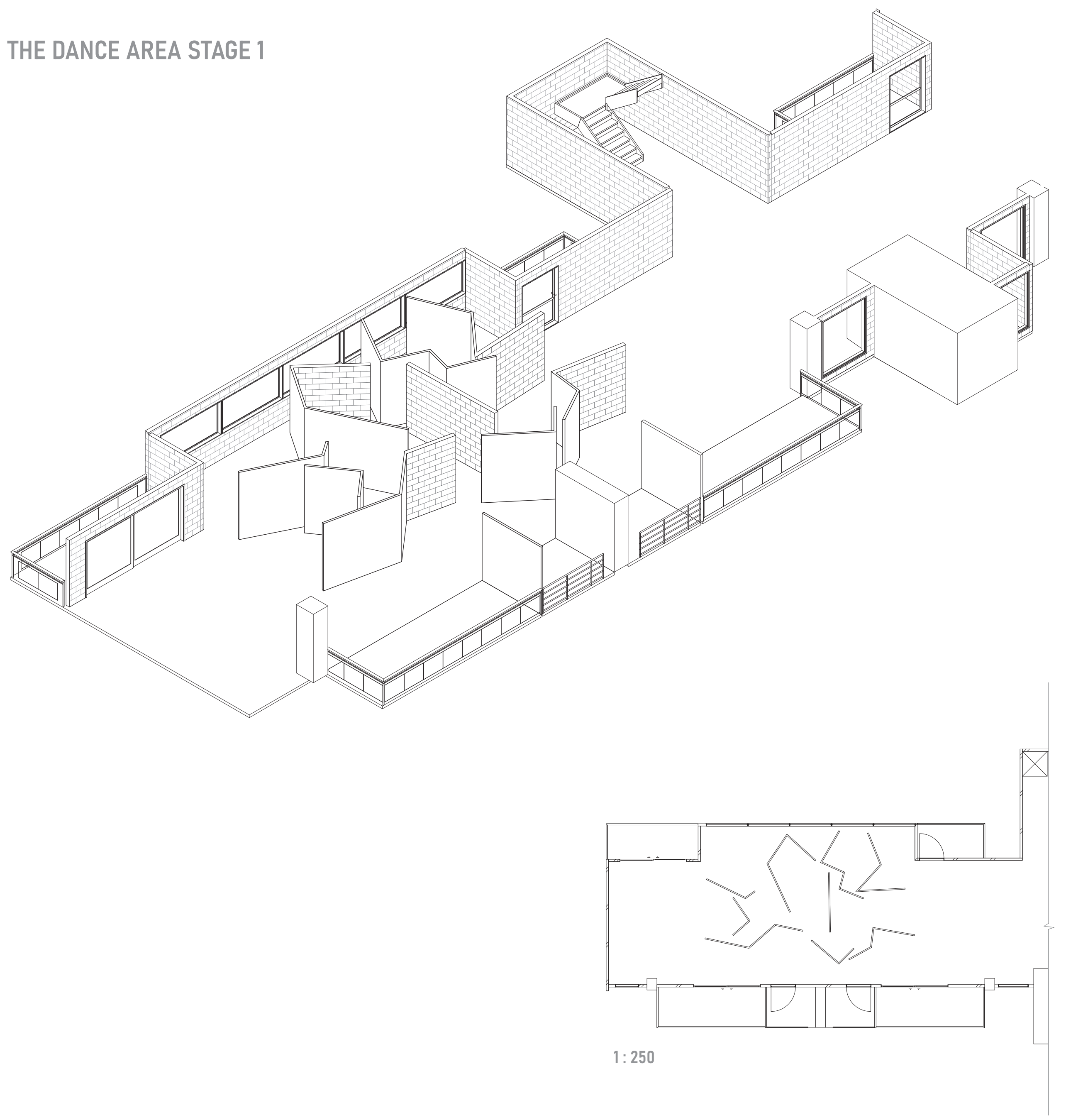



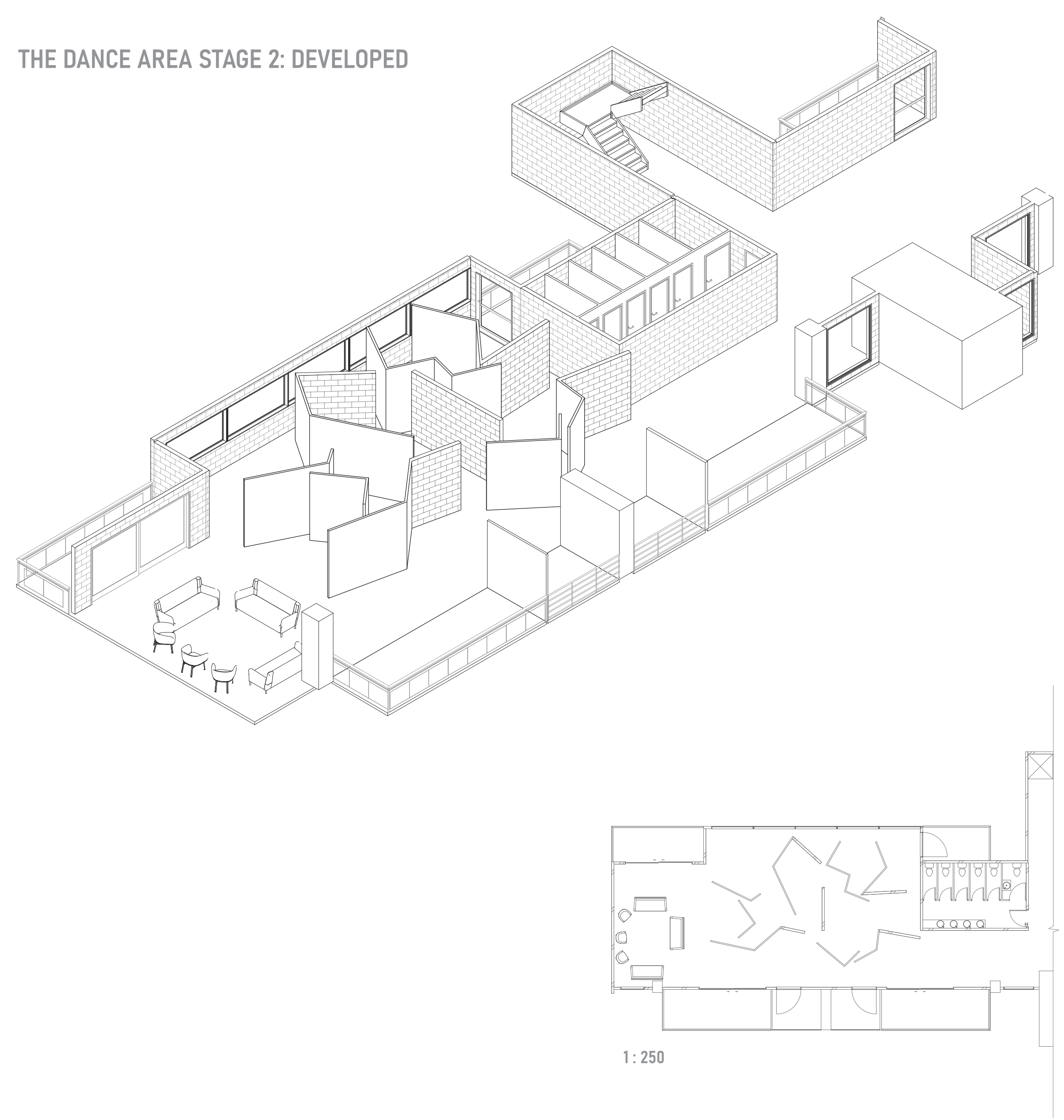


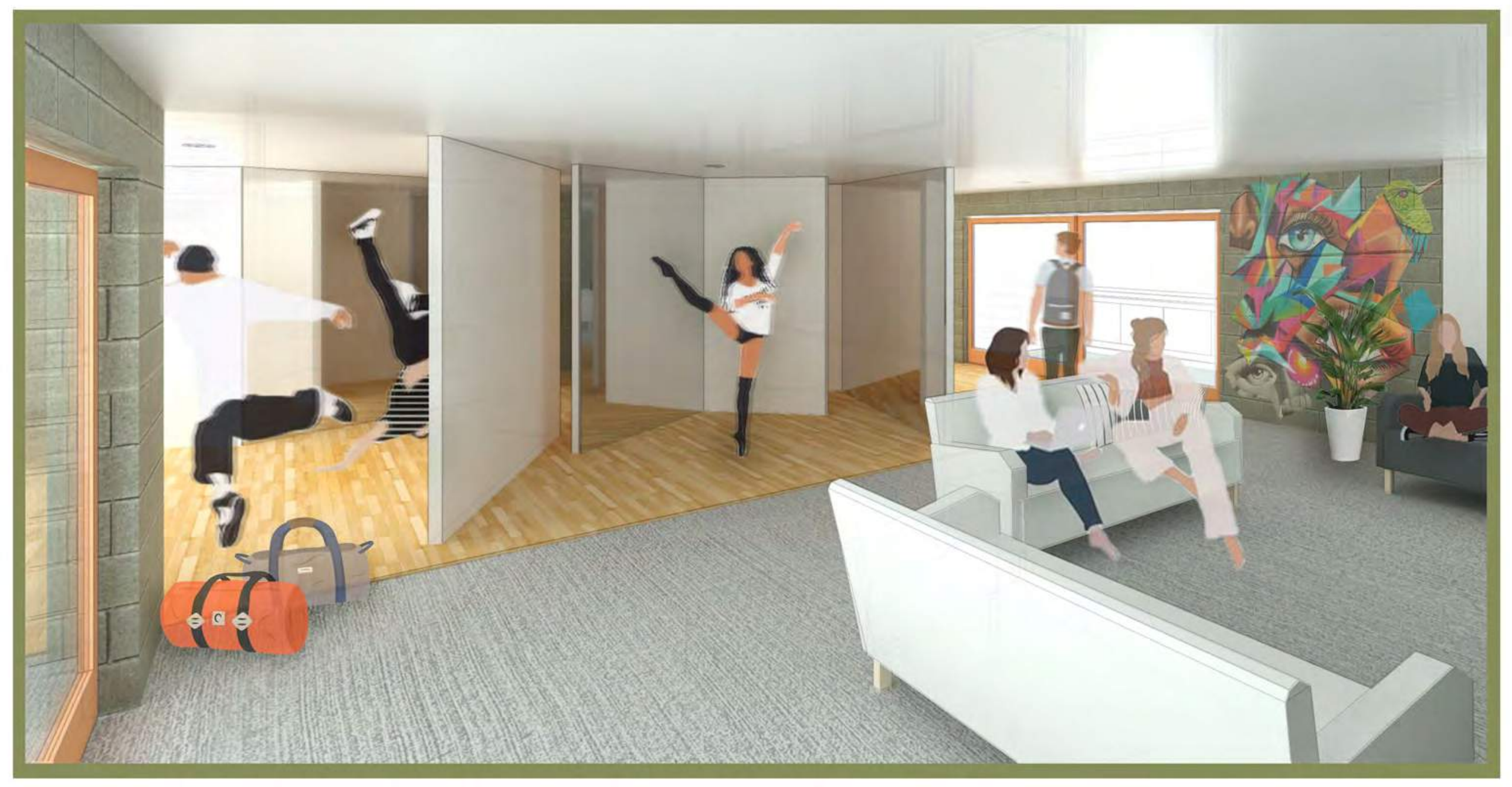

DANCE AREA + SEATING AREA 


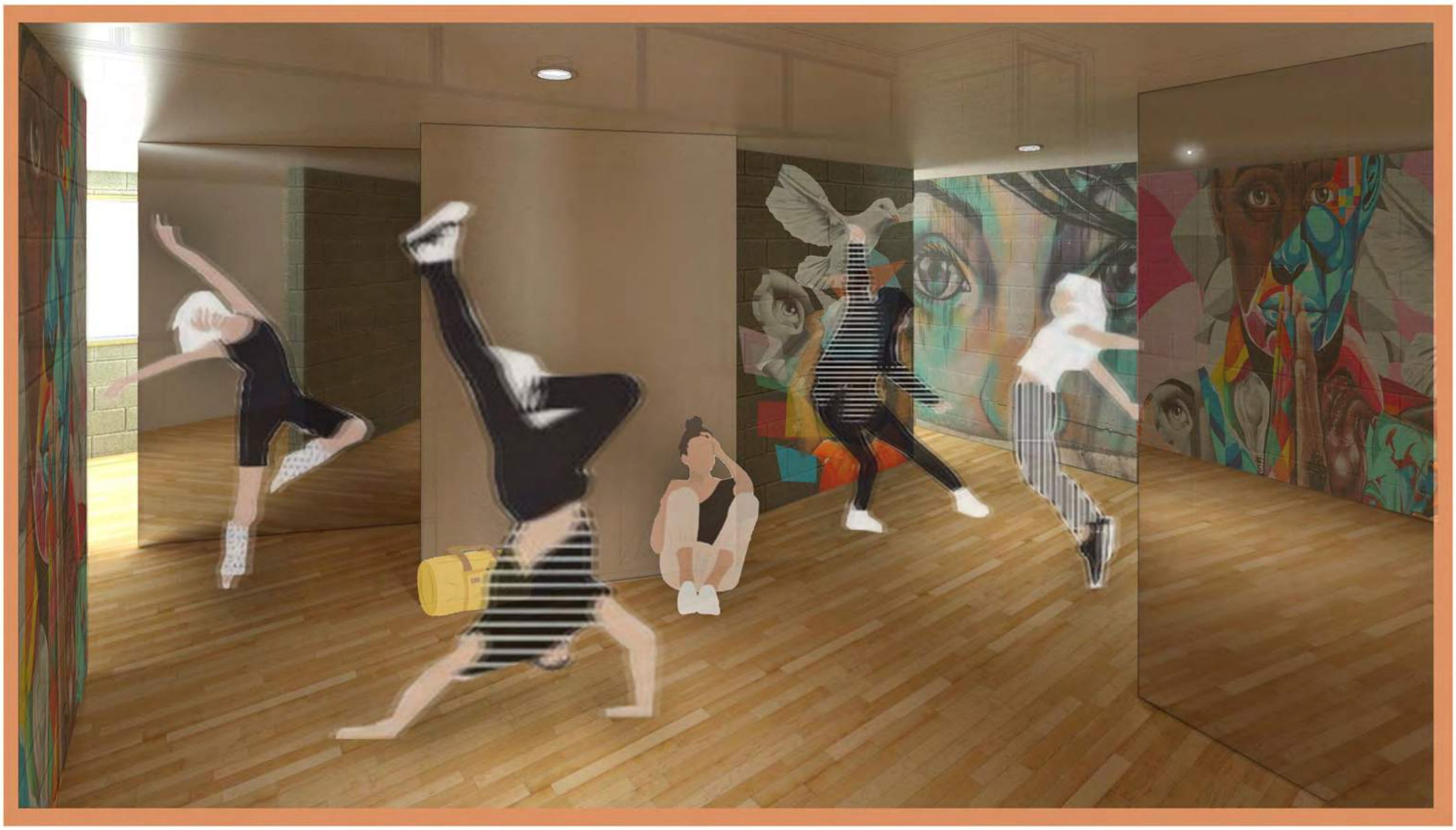

DANCE SPACE 
THE WORKSHOP / COMMUNAL SPACE
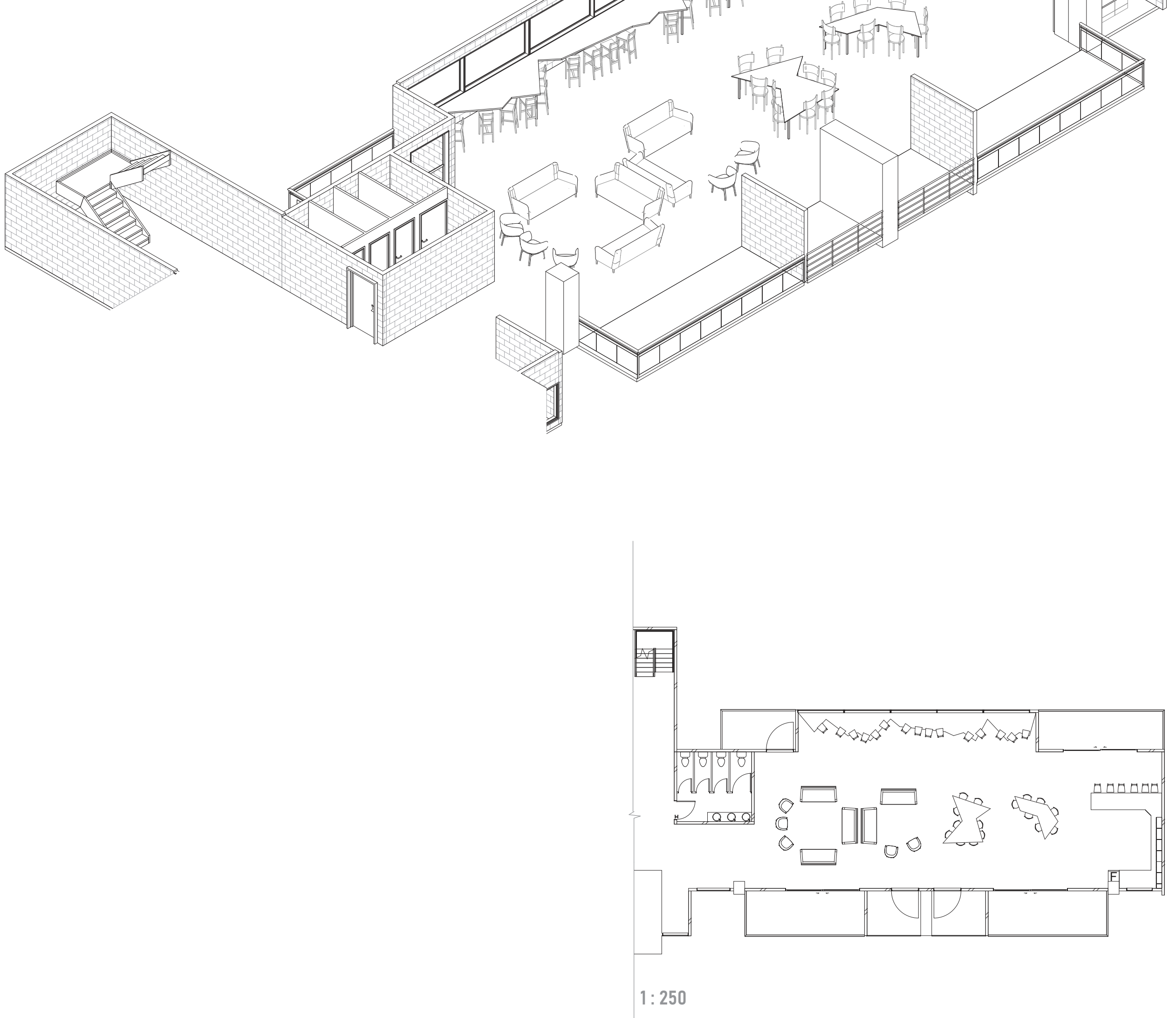


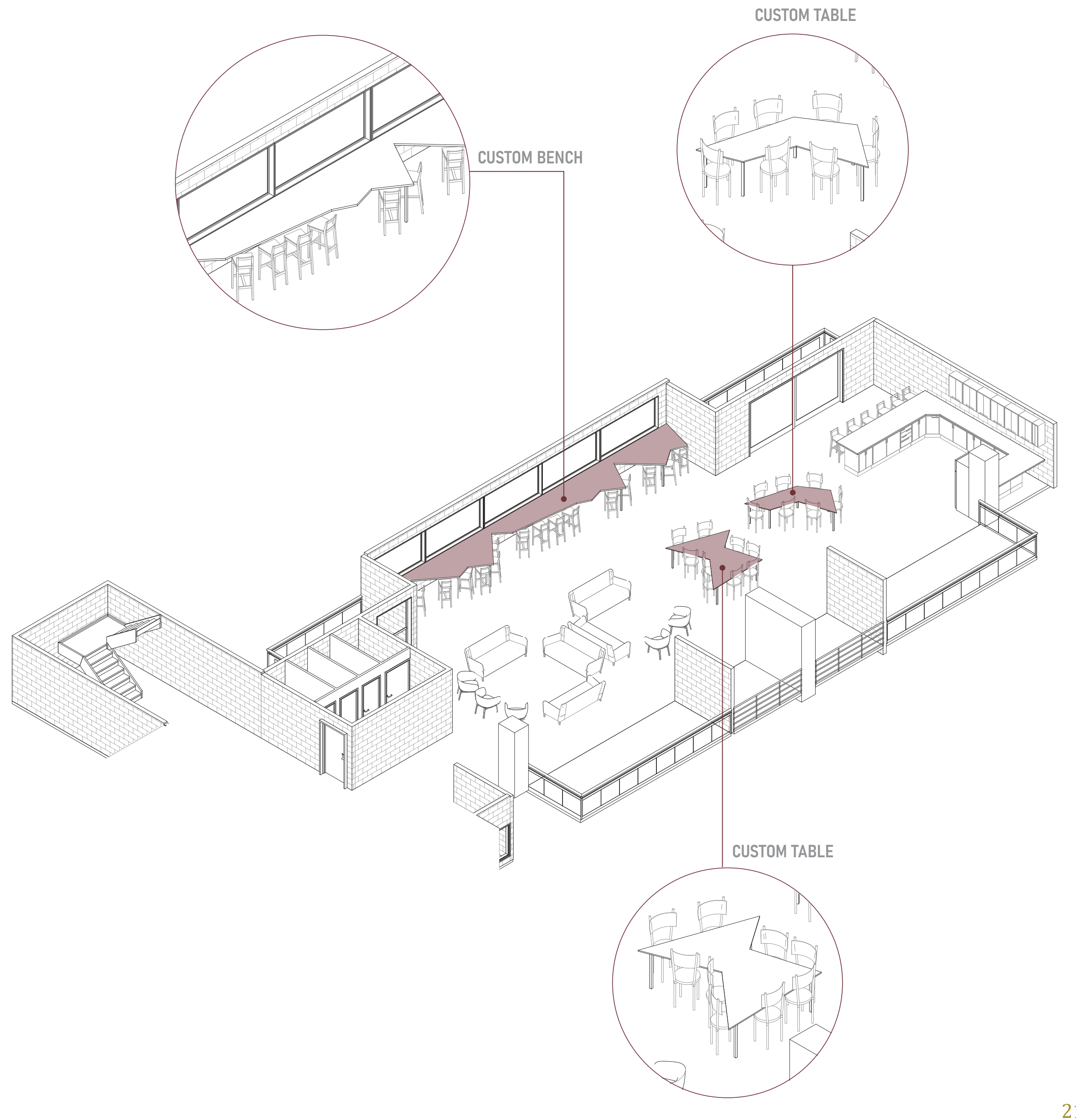

216 
SHAPE BEGINNING

\section{CUSTOM WINDOW BENCH}
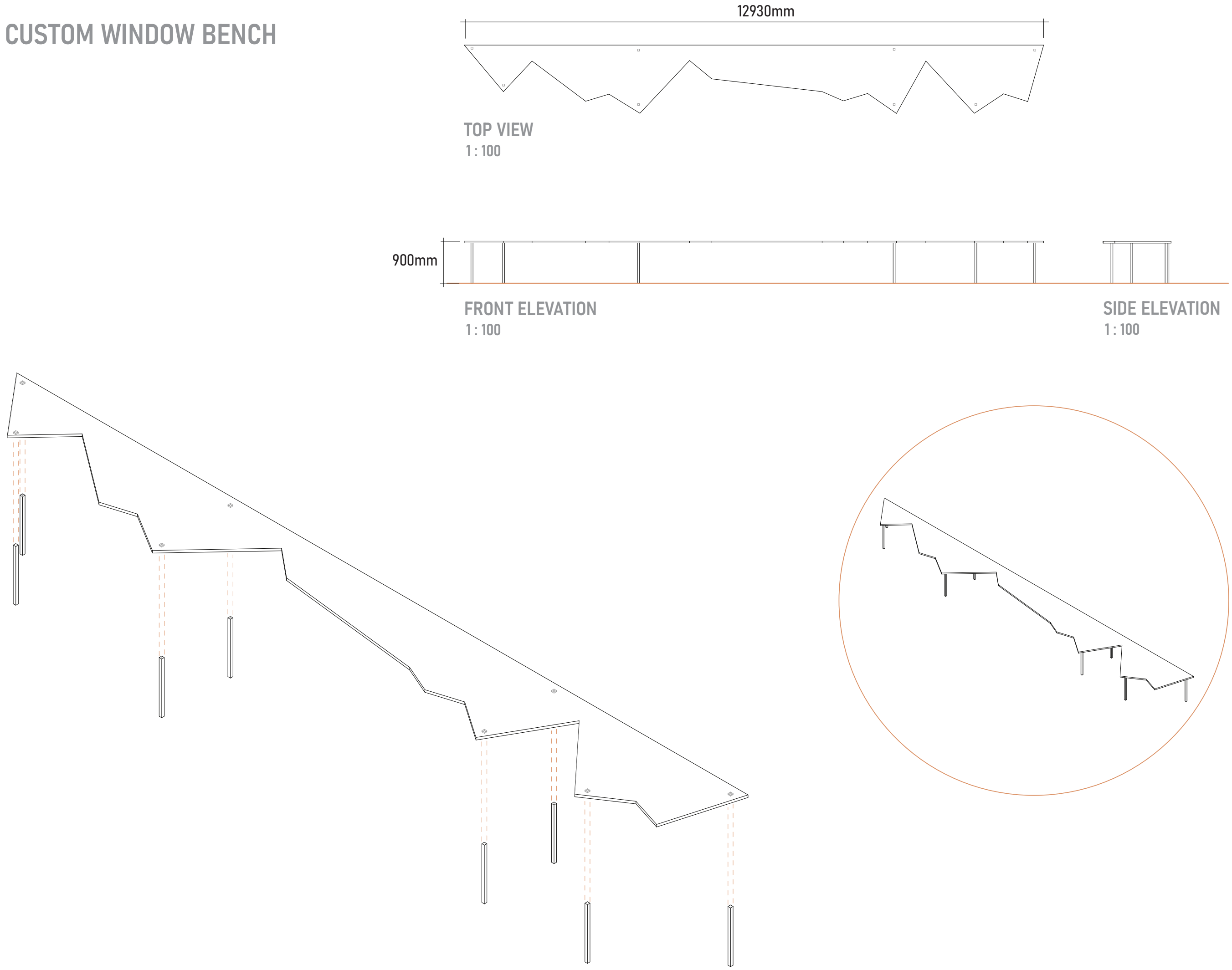
CUSTOM TABLES
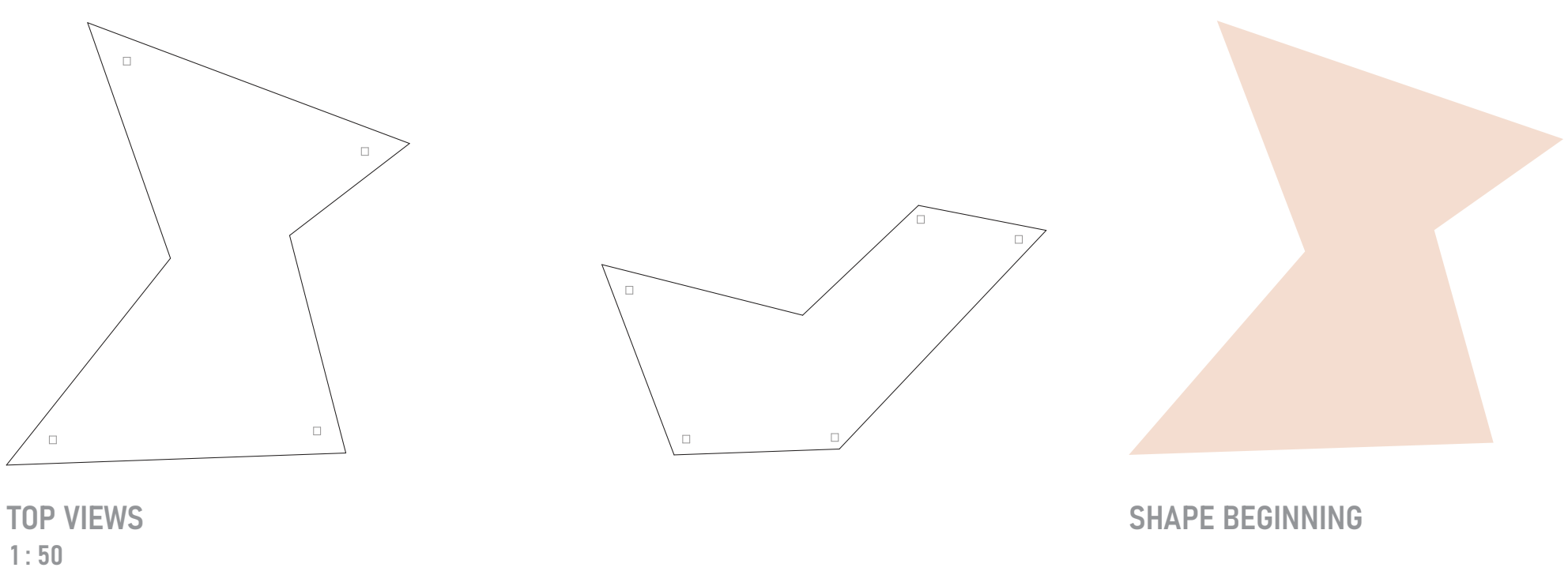

SHAPE BEGINNING

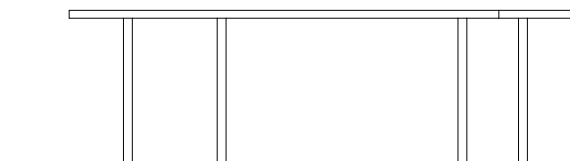

FRONT ELEVATIONS

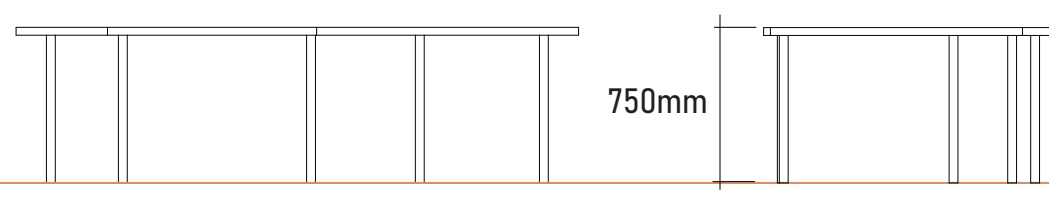

SIDE ELEVATIONS

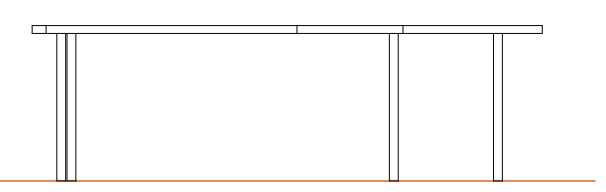

1: 50
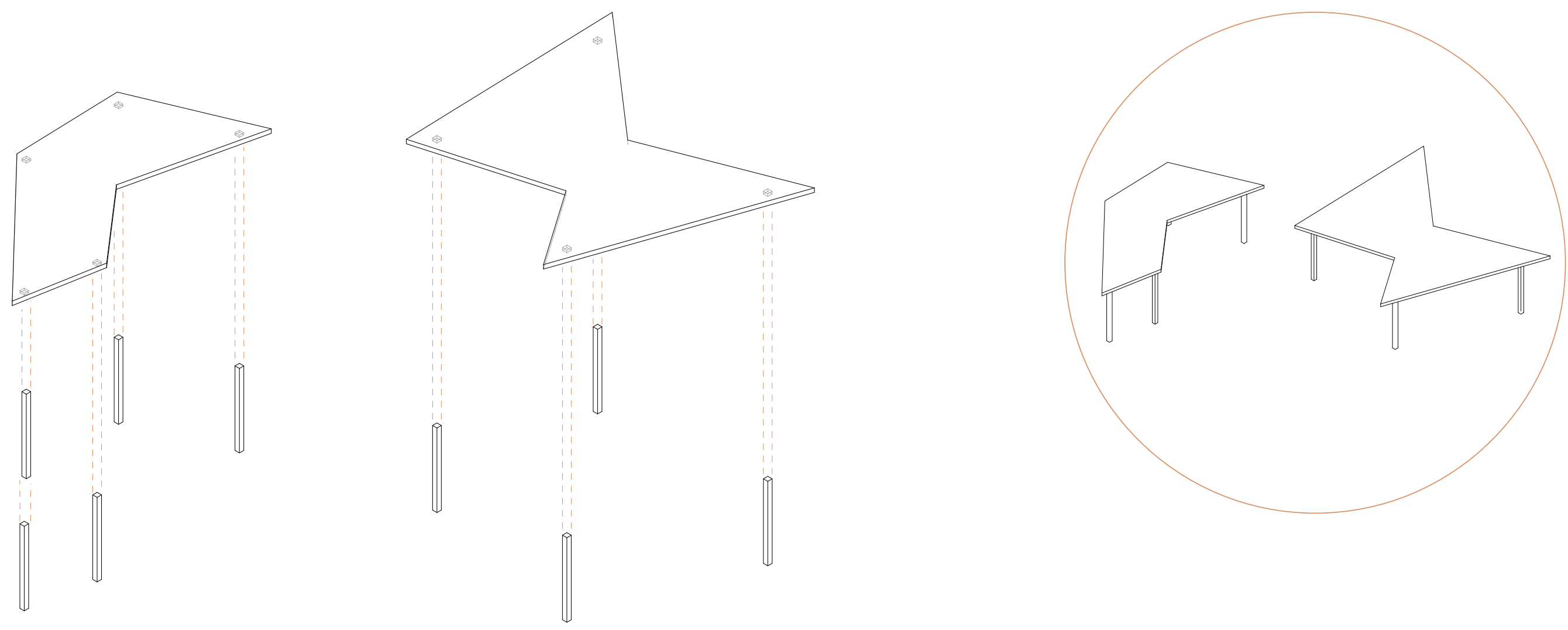

218 


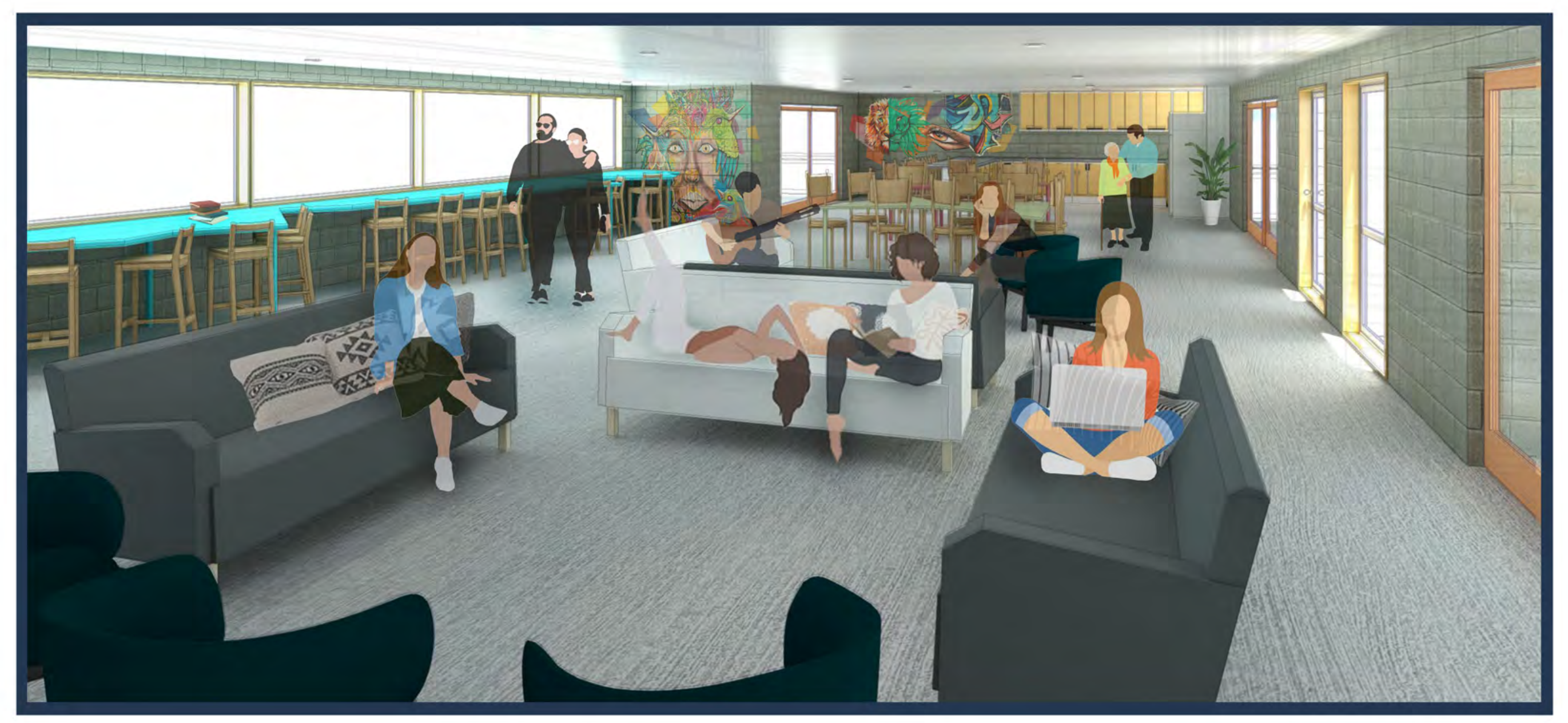

COMMUNITY + WORKSHOP SPACE 


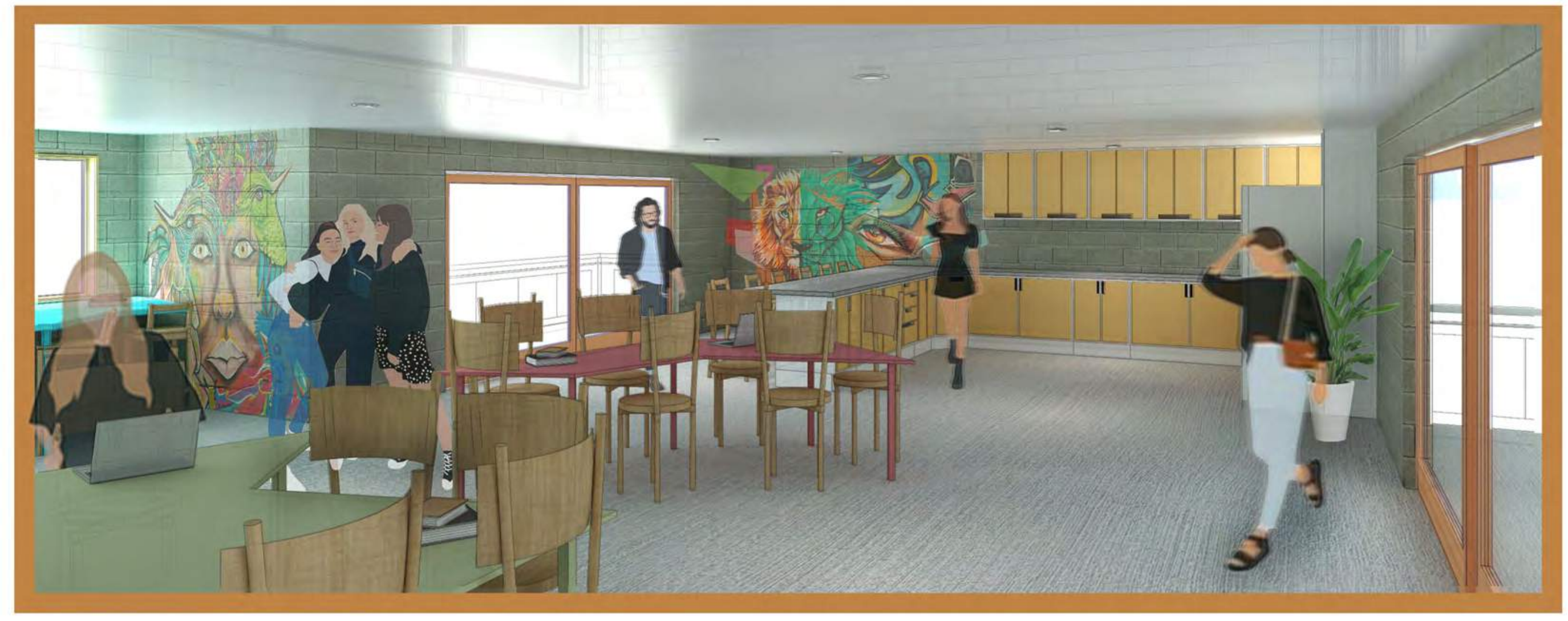

COMMUNITY + WORKSHOP SPACE 
FINAL FLOOR PLAN

5,545

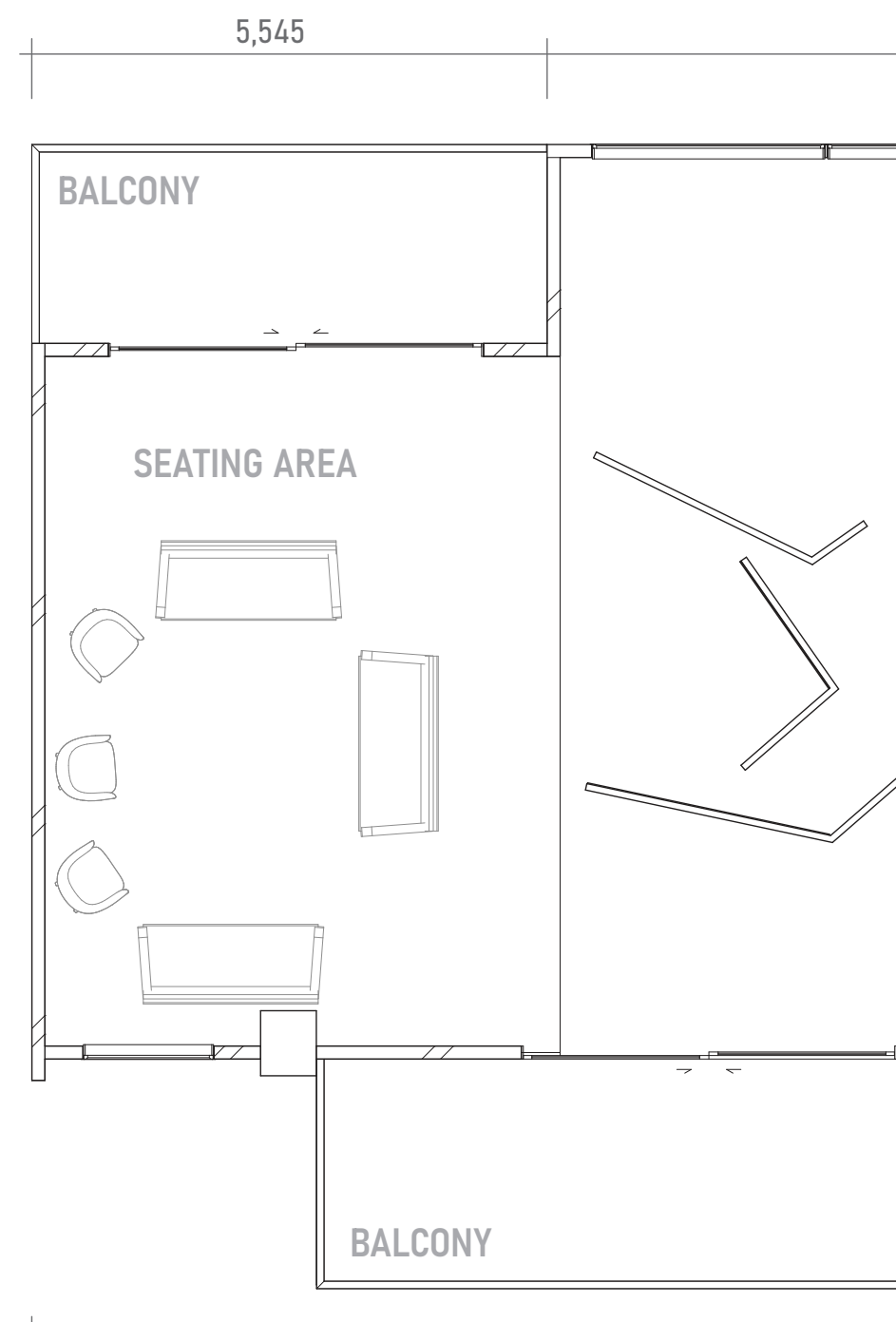

13,035

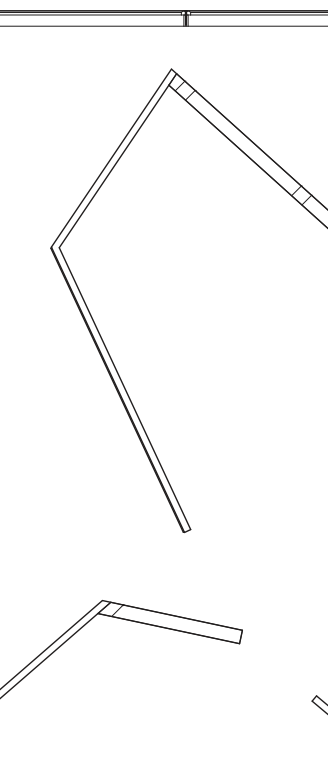

6,410

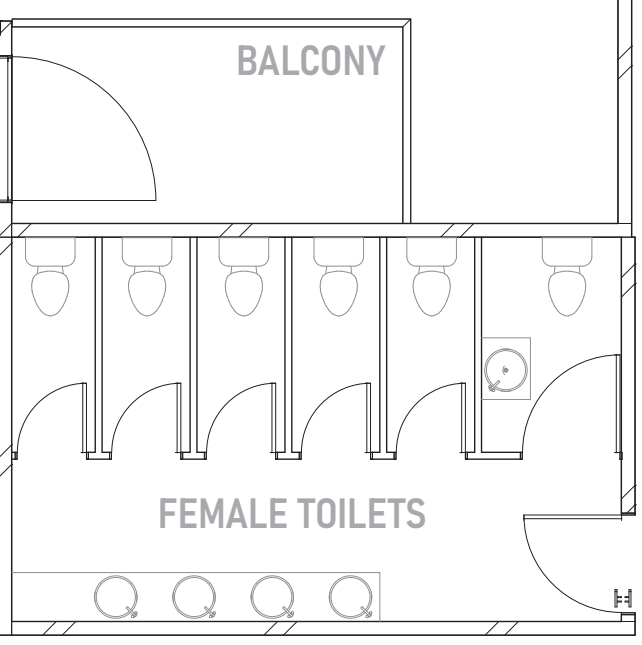

DANCE AREA
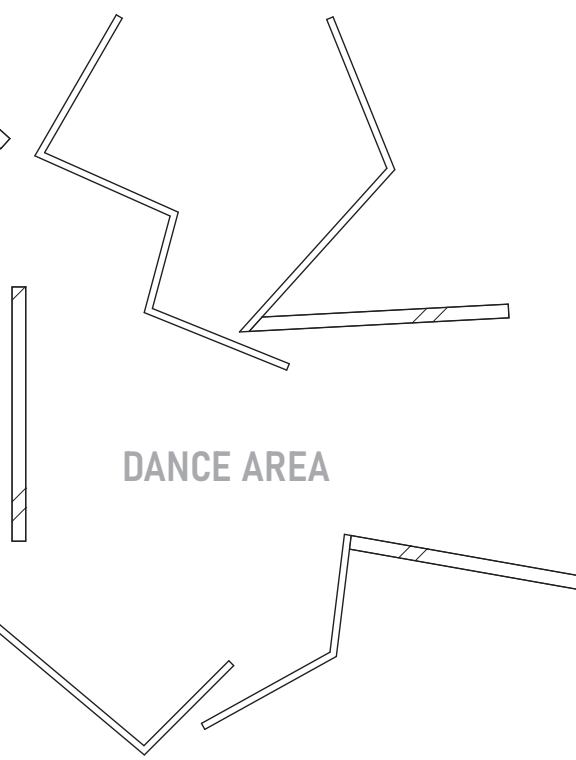
$=$

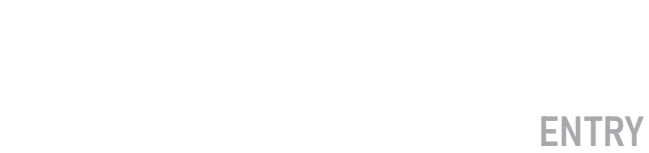

24,990

\section{DANCE STUDIO SIDE}

$1: 100$

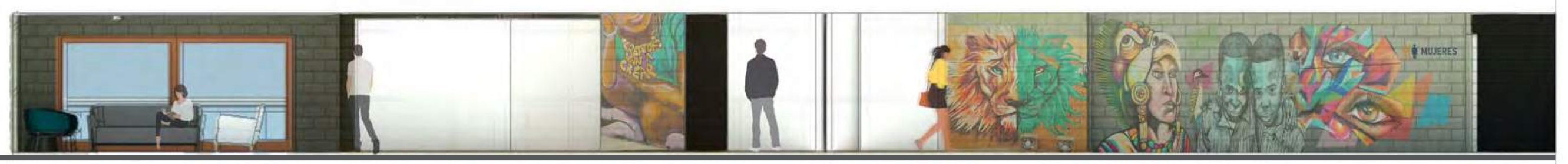

SECTION

$1: 100$ 


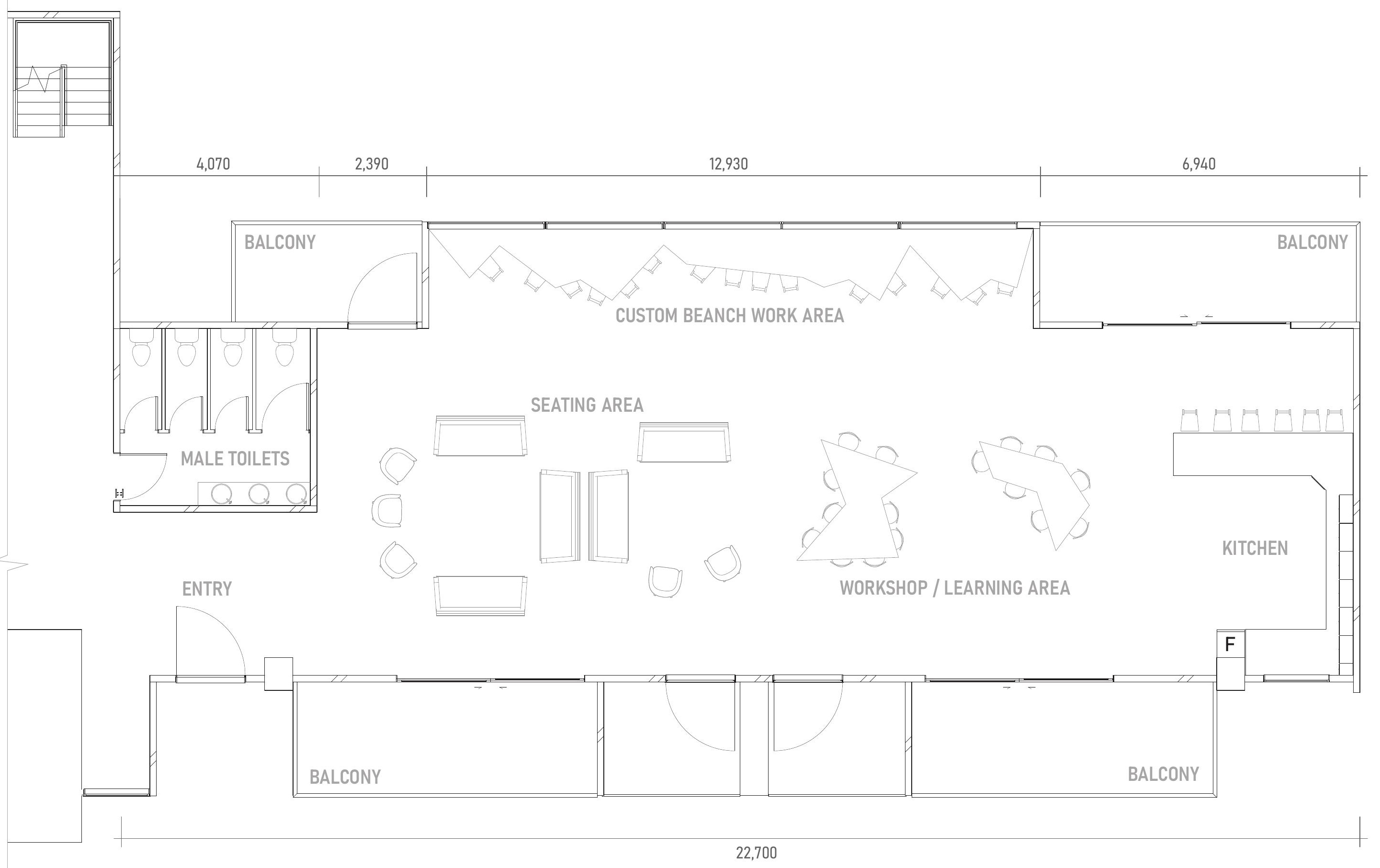

COMMUNITY WORKSHOP + SHARED SPACE

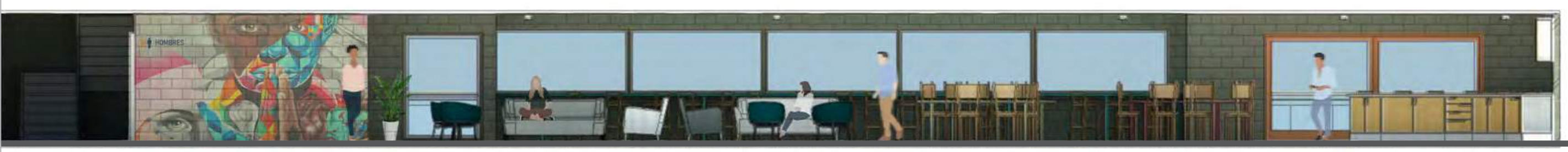


3. INTER-FLOOR COMMUNAL SPACE

ESPACIO COMÚN ENTRE PISOS 


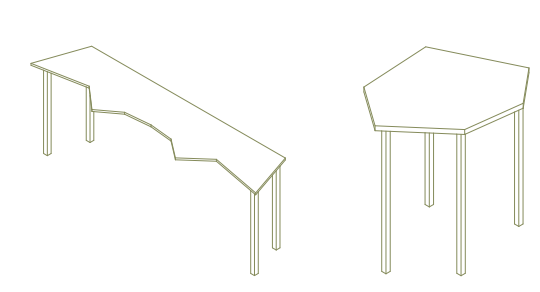


It is important to not only make the most of all space available throughout the building, but also to provide this community with as much space as possible to socialise and communicate.

This is why a communal space is created on every second level. This offers the opportunity for the formation of smaller communities within La Comuna 13 as the people from the six apartments on each floor can use this space to interact and spend time together. 
APARTMENTI

COMMUNAL

SPACE
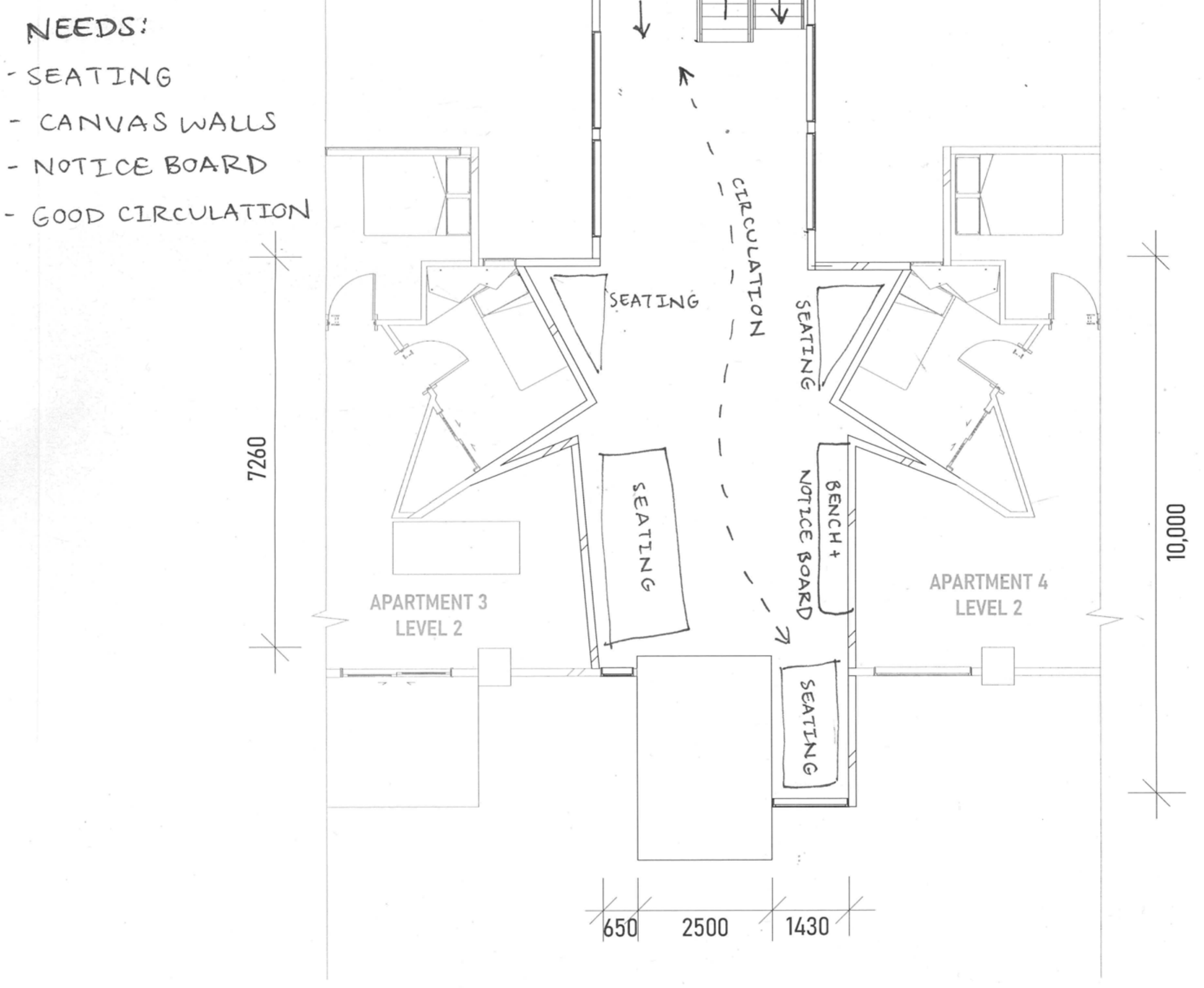

APARTMENT COMMUNAL SPACE PLAN

1:100 


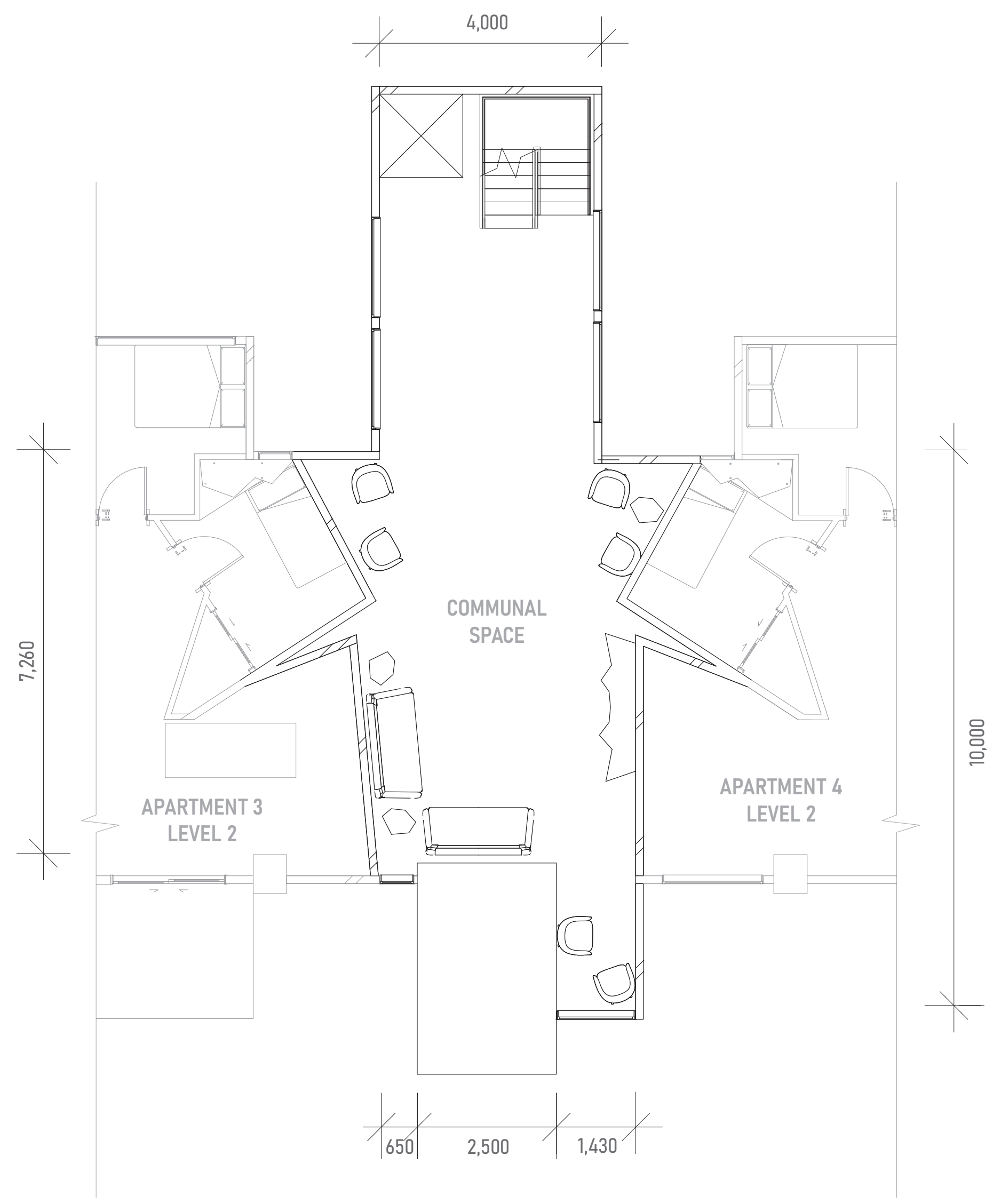

APARTMENT COMMUNAL SPACE PLAN

$1: 100$ 



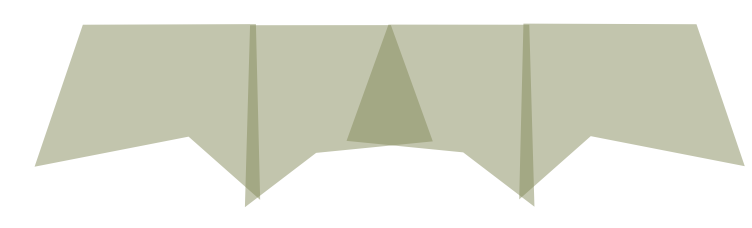

THE BEGINNING

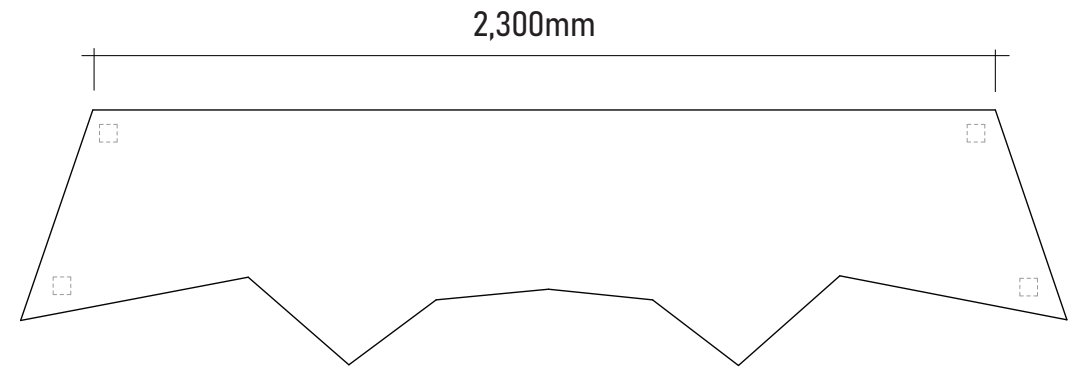

TOP VIEW

$1: 25$

$2,670 \mathrm{~mm}$

\section{CUSTOM BENCH / TABLE}
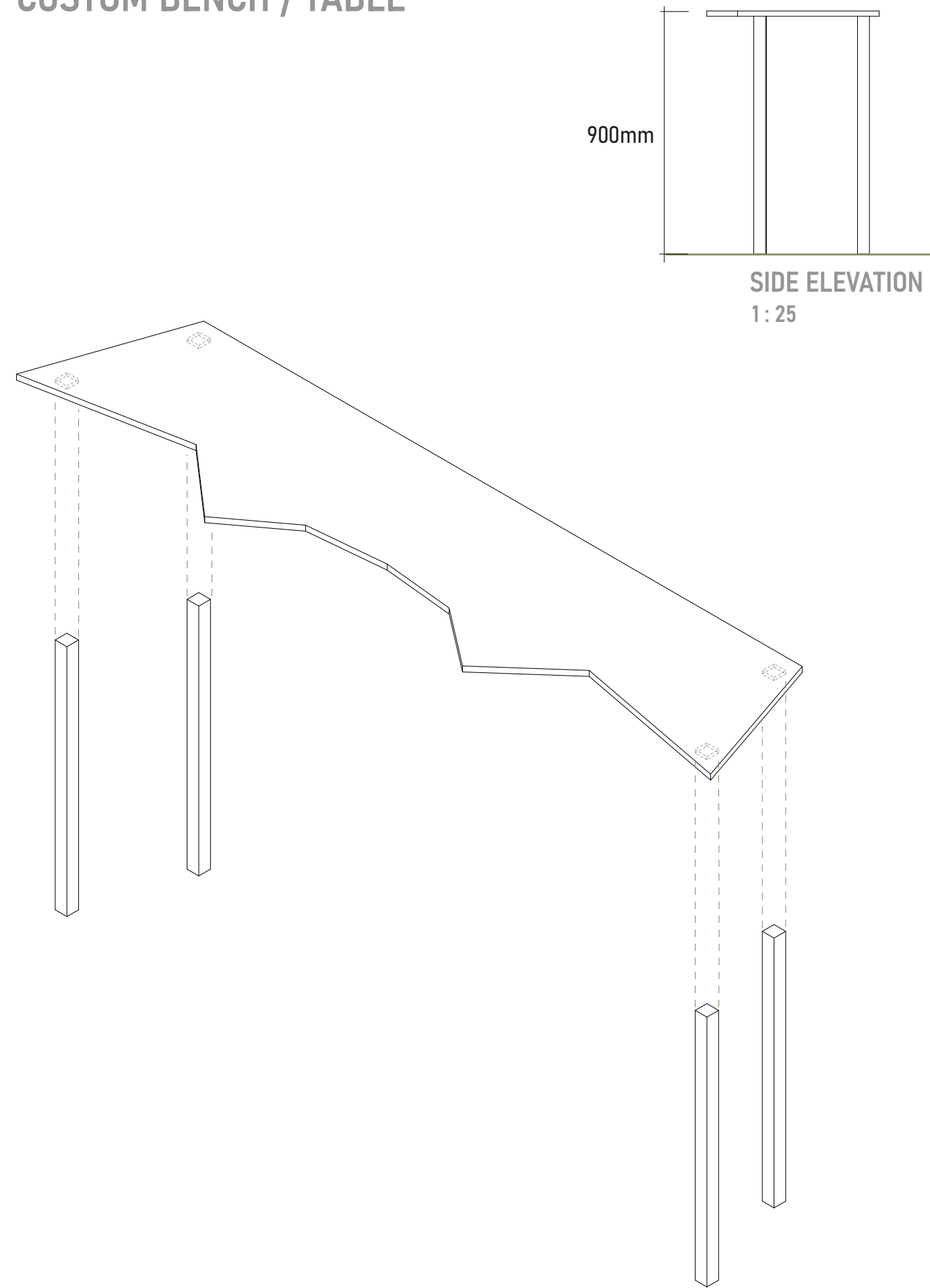

FRONT ELEVATION $1: 25$

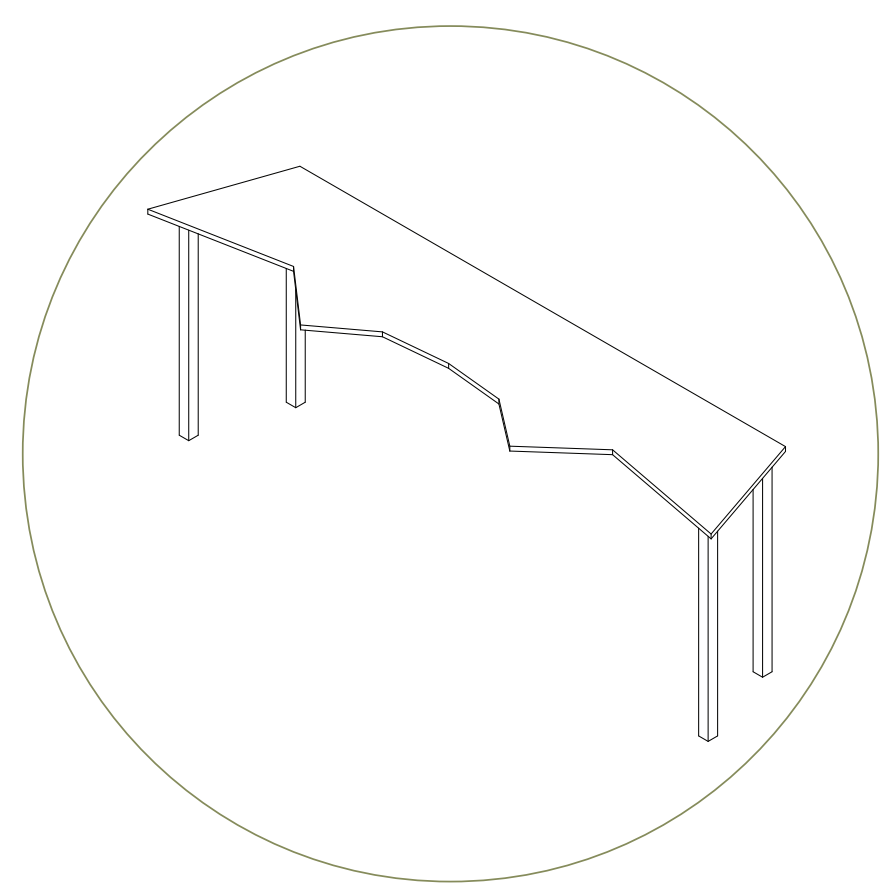




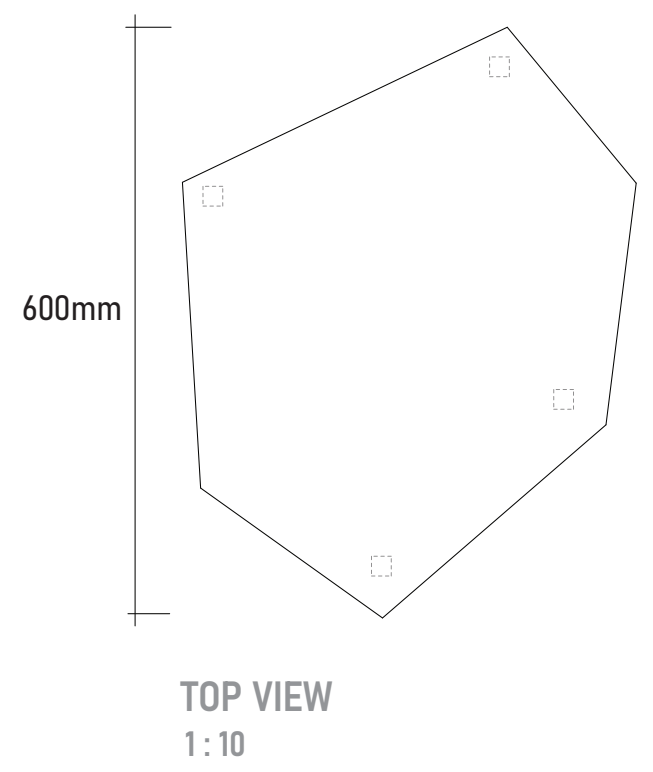

CUSTOM COFFEE TABLE

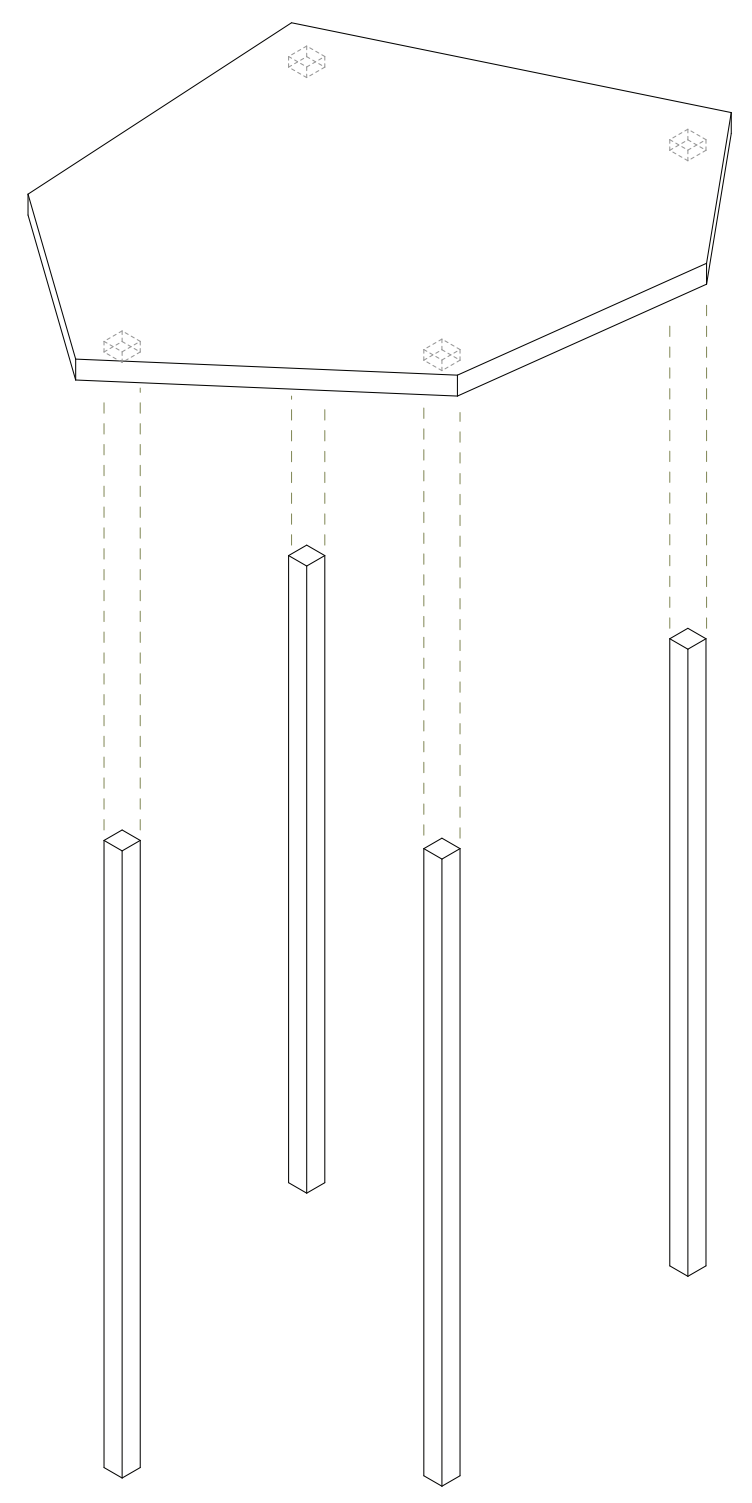

THE BEGINNING
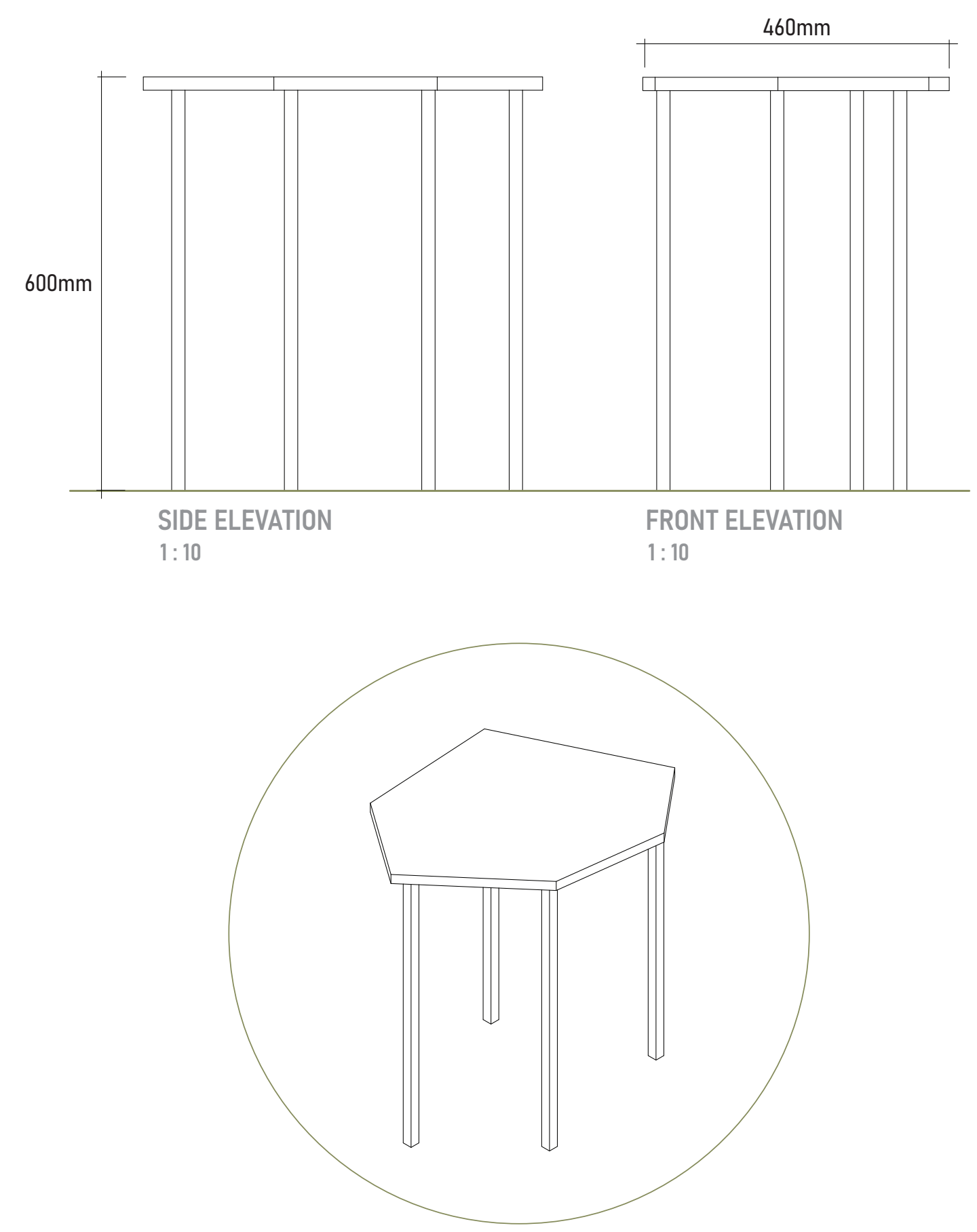

230 


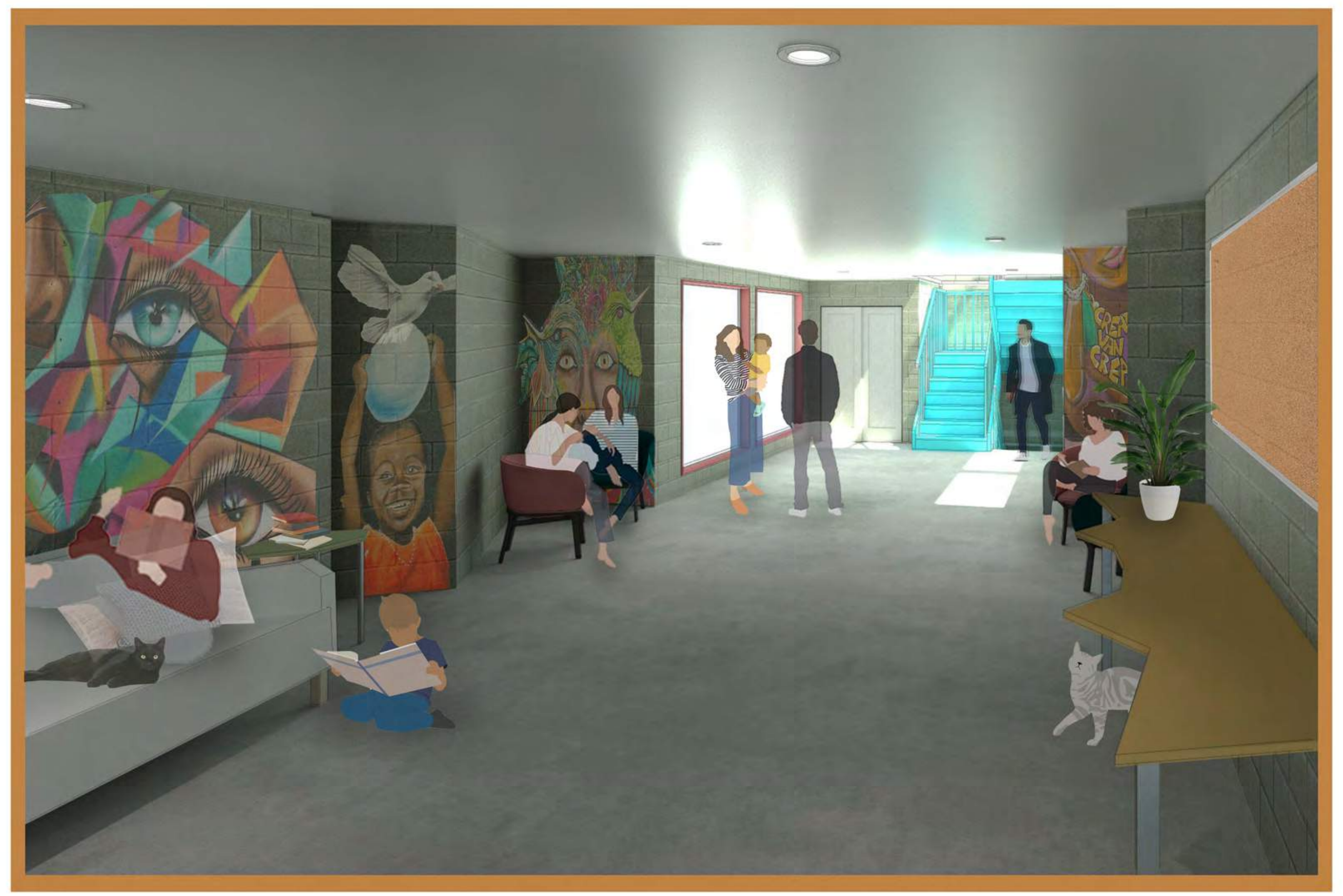

APARTMENT COMMUNAL SPACE 


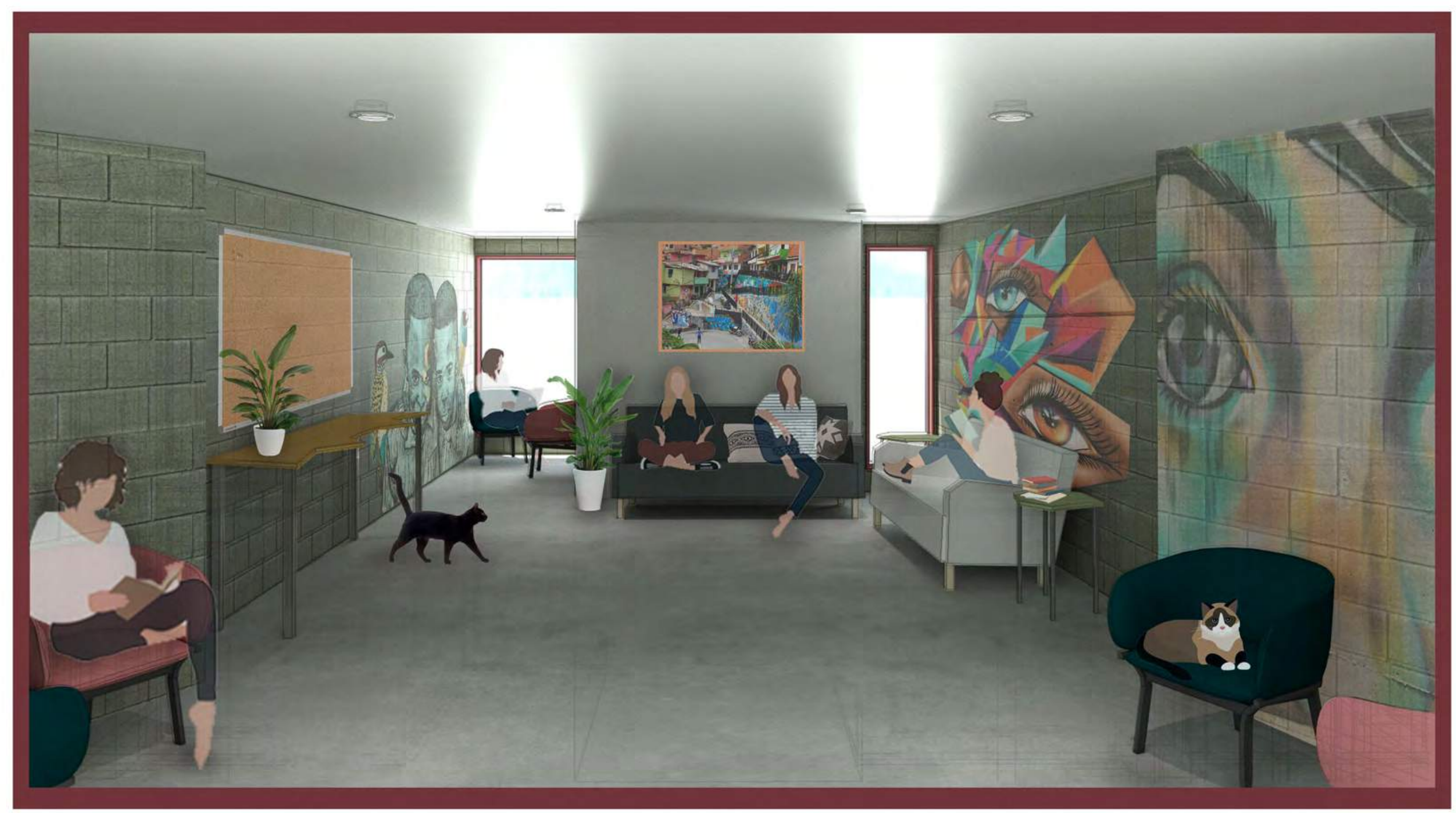

APARTMENT COMMUNAL SPACE 


\section{CONCLUSIONS + REFLECTIONS}

\section{Conclusiones + Reflexiones}


07 


\section{CONCLUSIONS}

Conclusiones

This thesis endeavoured to respond to the question of the role of interior architecture in enabling the expression of a community in the city and more precisely to find ways for an interior intervention to enable the expression of a marginal community throughout the urban fabric of mainstream culture.

The artistic expressions of a marginal community became the key connector to the different factors: community, culture, marginalisation, identity, urban environment and interior interventions, and to address their relationship.

This research originated from the realisation that in the city of Medellin, marginalised communities were not being expressed in the city centre. The rich art and culture which La Comuna 13 represents was being pushed to the hilly outskirts of the city and became saturated within just one community. This marginalisation began with a concentration of violence which occurred within just this community because of territory wars amongst guerrilla, paramilitaries and the government (from 1950-2010). The word spread quickly and the bad reputation of La Comuna 13 was imprinted in people's minds. It was known as a violent place; a place which should not be wondered into or even spoken of. Little did the city know, this community was healing its wound and embarking on a process of transformation through artistic expression. They used the walls in their streets to paint murals which represent their histories and memories from this time.

This thesis explores the position of interior architecture as a relevant discipline to further enable the expression of communities on the margins back into the urban centre of mainstream society. In order to do so, this research endeavoured to develop sim- ple design strategies where the interior architect uses the art produced by the marginal community as a design driver to create a new architecture. The intention was supported by the idea that such design development would not only responds to the original artistic expression but also aims to enable its expression within the city centre. By doing this, the marginalised community is being re-introduced to the city with a new positive reputation and active engagement in urban life.

This thesis speculates how the re-coding of the art produced within a certain community can be used to derive form and create an architecture which not only responds to this artistic expression but also serves to enable it. To test this, three different programmes were designed at a variety of scales. These programmes originated from the consideration of the types of spaces required by the forms of artistic expression occurring within this community such as painting, dancing and music. Due to the changing nature of these, the programmes differed significantly with the first programme tested being an urban installation, the second a pavilion/ dance studio and the third a high-density housing development within an abandoned building.

The link between the art being produced in this community and architecture became evident quite early in the research as art and architecture were linked by a key element; the wall. Timothy Horne's theories on the meaning and use of the wall were influential in the development of concepts. This idea of 'the wall' as a canvas became pivotal to the conceptual development throughout the design of the three programmes.

The first programme which was an urban installation used this idea in its most ba- sic form. It was an instalment of walls on a busy street which the community could use to paint murals on and be seen by the wider public. At a conceptual stage, the design worked as the walls were all made of brick to replicate their current canvases and they were oriented in all different directions as a response to the re-coding of the art. If this programme were to be developed further, there is room to continue to improve aspects such as circulation and occupation of the space. This idea could also be pushed further by creating multiple of these installations and placing them throughout the city to increase the exposure of the community. As a starting concept, this became essentially an urban gallery for this community to be displayed to the public. By placing it in people's path, they were forced to engage and become aware. A key learning from this stage was the importance of the wall and the continued use of this architectural element to enable the artistic expression.

At the beginning of conceptualising the second programme exploration, it became evident that this community's artistic expression was not strictly limited to painting. Dancing on the streets for tourists is one way in which the young people within this community earn money and so it would have been inappropriate to ignore the art of dance throughout conceptualisation. The research done by Bloomer and Moore was critical in the understanding of the relationship between dance and architecture and how much one can influence the other. Using the learnings from the first concept around the idea of the wall as a canvas and this consideration towards dance, the second concept was born; a pavilion in the city centre which would be a dance studio for this community. This programme almost added another layer onto the previous as it still had the brick walls for the murals but 
it was now also a space which enabled another form of art as well. It is important to note that while this programme was largely about the interior space, there was an architectural element to it. If this programme was to be further developed, there would be possibilities for the refinement of the envelope of the pavilion and how it responds to its context. There were some considerations made in this regard in this research but they could be pushed much further and even used to develop the architecture. This was not the focus here and the focus was situated around materiality, atmospheric and spatial qualities on the inside.

The final programme originated from something quite different to the first two. The urban gallery and the dance pavilion were both concerned with creating a space for this community to be able to express themselves within the city centre. However, through a deeper understanding of La Comuna 13, it became clear that what this community truly wants is to move out of their current housing and into a place with better opportunities and accessibility to the city. This is where the idea to create high density housing emerged. The goal was to continue to use the previous learnings of the wall as a canvas and the art of dance to develop housing which truly catered to this community's needs. With sustainability at the forefront, it was decided to use an old abandoned building and simply focus on the interior architecture. This programme essentially became a culmination of the previous explorations whilst introducing the element of housing.

Of the three concepts, the author believes the third encounter was most successful in terms of achieving the aims and objectives of this design-led research. The programme housing not only reflected all the key themes and ideas which were significant throughout the design process but it also address the current issue of this community wishing for a better living situation. Even though this research focused on exposing the community to the public, the most hidden encounter became the most successful one.

Using the criteria of exposure to analyse the success of the programmes it could be argued that the Invited Encounter was most successful. In comparison to the Forced Encounter, the Invited encounter not only embodies the ideas of layering and place-identity established in the aims of this research but it also more accurately represents $\mathrm{La}$ Comuna 13. There is a culture within La Comuna 13 of openness and inviting the public to see what they are doing and creating. The dance pavilion better reflects this aspect of allowing the public to decide when and how they would like to engage with the art and the community members. The Forced Encounter whilst powerful in creating exposure, is perhaps too intense as it simply demands that the public engages with the art rather than offering them a choice. 


\section{FINAL REFLECTIONS}

Reflexiones finales

It is important to note that the intention of this research is not to appropriate the art being produced by La Comuna 13 but to recode it. The idea is to create spaces which are a response to the art and enable its creation through spatial qualities and materiality. Even though this inquiry is largely based around interior architecture strategies, there are elements in the design which push the idea of interiority and its preconceived boundaries: an urban installation, and an architectural object (a pavilion within an urban setting) are the first 2 iterations.

This design-led research aimed to reflect ideas of layering, memory and place-identity which is something that has been achieved in a range of ways throughout. Whether it be through the literal layering of architectural elements or the layering of the art on the walls. The understanding that this original art archives the memories of a community and is to be acknowledged. As well as the constant desire during the design process to create spaces which truly belong to such community; spaces which they could identify with and wishing to inhabit.
The author hopes that this design-led research will inspire other designers to engage with culture and communities via a similar approach. Other processes could be invented for other requirements and site specificities; this is inevitable. This work has developed through discoveries that lead to the idea of art re-coding and its iterative testing at different scales and programmes. A mysterious event towards the end of this research lead to the author directly connecting with La Comuna 13; a reaffirming of some sort of the worth of the endeavour. The author hopes that this work will remain as not only a personal connection to her own culture but as a process of honouring a resilient people and their artistic expression through the means of her own discipline of interior architecture. 


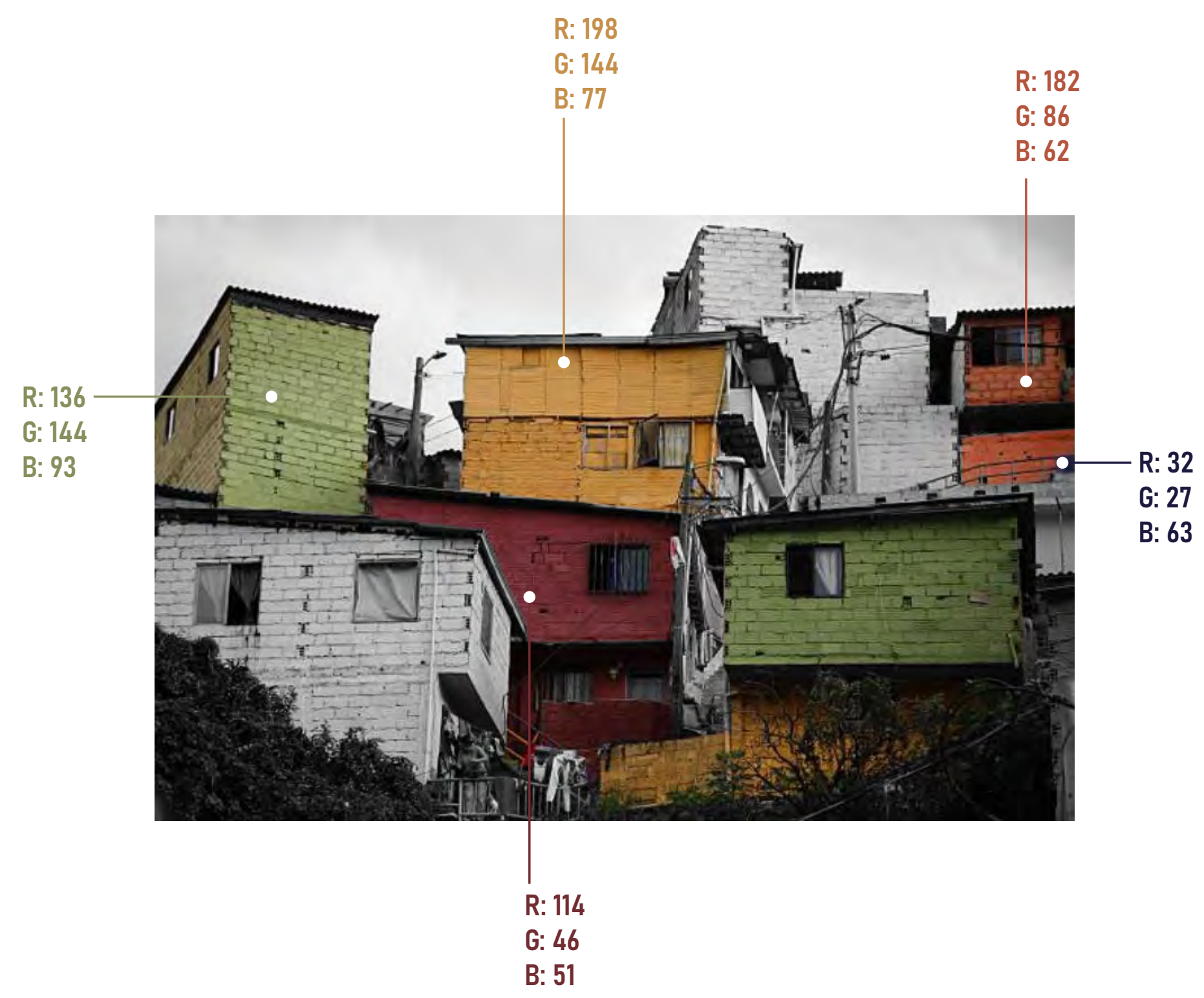




\section{BIBLIOGRAPHY}

Bibliografía

Ashworth, G., Graham, B., \& Tunbridge, J. (2007). Pluralising pasts. Heritage, Identity and Place in Multicultural Societies.

Bastéa, E. (2004). Memory and Architecture. (pp. 1-335). Albuquerque: University of New Mexico Press.

Bitter, S., \& Weber, H. (2018). MAKING RUINS. In P. TORTELL, M. TURIN, \& M. YOUNG (Eds.), Memory (pp. 39-48). Peter Wall Institute for Advanced Studies; JSTOR. https://www.jstor.org/stable/j.ctvbtzpfm.7

Bloomer, K. C. (1977a). Body, memory, and architecture. Yale University Press.

Bloomer, K. C. (1977b). Body, memory, and architecture. Yale University Press.

Brooker, G. (2016). Interior Matter(s): Proximities, Inhabitation, Identities: Interior Matter(s). Journal of Interior Design, 41(4), 5-12. https://doi.org/10.1111/joid.12084

Bullen, P., \& Love, P. (2011). A new future for the past: A model for adaptive reuse decision-making. Built Environment Project and Asset Management; Bingley, 1(1), 32-44. http://dx.doi.org.helicon.vuw.ac.nz/10.1108/20441241111143768

Ching, F. D. K., \& Ching, F. (2014). Architecture: Form, Space, and Order. John Wiley \& Sons, Incorporated. http://ebookcentral. proquest.com/lib/vuw/detail.action?docID $=1771578$

Davis, M. (1984). The Layering of Form and Space in Interior Architecture. Victoria University of Wellington. https://tewaharoa. victoria.ac.nz/discovery/fulldisplay?docid=alma99179002815302386\&context=L\&vid=64VUW_INST:VUWNUI\&lang=en\&search_scope=MyInst_and_CI\&adaptor=Local\%20Search\%20Engine\&tab=all\&query=any,contains,layering\%20AND\%20architecture\&offset $=0$

Drummond, H., Dizgun, J., \& Keeling, D. J. (2012). Medellín: A City Reborn? Focus on Geography, 55(4), 146-154. https://doi. org/10.1111/foge.12005

goldberger, paul. (2009). Architecture and memory. In Why Architecture Matters (pp. 139-170). Yale University Press; JSTOR. https://www.jstor.org/stable/j.ctt1nq221.8

Ho, T. (1975). Design criteria for human high-density housing. Ekistics, 39(235), 377-381. JSTOR.

Horne, T. (1989). The wall in contemporary architecture [Victoria University of Wellington]. https://viewer.waireto.victoria. ac.nz/client/viewer/IE944543/details?dps_dvs=1589331376287 592

Lanz, F. (2018). Re-Inhabiting. Thoughts on the Contribution of Interior Architecture to Adaptive Intervention: People, Places, and Identities. Journal of Interior Design, 43(2), 3-10. https://doi.org/10.1111/joid.12121 
Lush, E. (2020, February 12). 13 Things to Consider Before You Book a Comuna 13 Tour in Medellin. Wander-Lush. https://wander-lush.org/comuna-13-tour-tips/

Mackenzie, N. (2018, September 10). The Inspirational Street Art of Comuna 13, Medellin. Above Us Only Skies. https://www. aboveusonlyskies.com/street-art-comuna-13-medellin/

Moussavi, F. (2007). Hybrid Identities: Mutating Type. Log, 10, 81-35. JSTOR.

Naef, P. (2018). Touring the 'comuna': Memory and transformation in Medellin, Colombia. Journal of Tourism and Cultural Change, 16(2), 173-190. https://doi.org/10.1080/14766825.2016.1246555

Neiza, L., Patino, L., \& Gonzales, M. (2017). Estructura y dinamica en familias de un sector rural de la zona norte de Bogota: Aportes para el desarrollo. In S. Idrovo-Carlier \& C. Torres-Castillo (Eds.), El lado humano de la sostenibilidad (1st ed., pp. 67120). Universidad de la Sabana; JSTOR. https://www.jstor.org/stable/j.ctvn1tbvn.6

Perez-Perez, A. L. (2015). El diseno de la vivienda de interes social. Revista de Arquitectura, Vol. 18 (ene-jun. 2016$),$ p.p 67-75.

Proshansky, H. M., Fabian, A. K., \& Kaminoff, R. (1983). Place-identity: Physical world socialization of the self. Journal of Environmental Psychology, 3(1), 57-83. https://doi.org/10.1016/S0272-4944(83)80021-8

Rolston, B., \& Ospina, S. (2017). Picturing peace: Murals and memory in Colombia. Race \& Class, 58(3), 23-45. https://doi. org/10.1177/0306396816663387

Schittich, C. (2012). High-Density Housing: Concepts, Planning, Construction. Walter de Gruyter.

Tice, J. (1993). Theme and Variations: A Typological Approach to Housing Design, Teaching, and Research. Journal of Architectural Education (1984-), 46(3), 162-175. JSTOR. https://doi.org/10.2307/1425158

Venturi, R. (1977). Complexity and contradiction in architecture (2d ed.). Museum of Modern Art.

Weinthal, L. (2018). A pirouette on the orthographic hinge. In The Interior Architecture Theory Reader. Taylor \& Francis Group. http://ebookcentral.proquest.com/lib/vuw/detail.action?docID=5404259

Wong, L. (2016). Adaptive Reuse: Extending the Lives of Buildings. Walter de Gruyter GmbH. http://ebookcentral.proquest.com/ lib/vuw/detail.action?docID $=4793914$ 
Lista de figuras

All figures not numbered and Figures 10,11 and 12 are all author's own.

Figure 1: Houses in La Comuna 13

Source: https://www.gettyimages.com.au/photos/comuna-13?mediatype=photography\&page $=4 \&$ phrase $=$ comuna\%20 13\&sort=mostpopular

Figure 2: Mural in La Comuna 13

Source: https://verdadabierta.com/nosotras-somos-guerreras-la-verdad-la-paz-lideresas-la-comuna-13/galeria-comuna-13-2/

\section{Figure 3: Mural}

Source: https://www.gettyimages.com.au/photos/comuna-13?mediatype=photography\&page $=4 \&$ phrase $=$ comuna\%20

13\&sort=mostpopular

Figure 4: Mural

Source: https://www.gettyimages.com.au/photos/comuna-13?mediatype=photography\&page=4\&phrase=comuna\%20 13\&sort=mostpopular

Figure 5: Peacock mural

Source: https://www.aboveusonlyskies.com/street-art-comuna-13-medellin/

Figure 6: Soldier on the street

Source: https://www.gettyimages.com.au/photos/comuna-13?mediatype=photography\&page $=4 \&$ phrase $=$ comuna\%20 13\&sort=mostpopular

Figure 7: Woman walking

Source: https://www.gettyimages.com.au/photos/comuna-13?mediatype=photography\&page $=4 \&$ phrase $=$ comuna\%20 13\&sort=mostpopular

Figure 8: Young man with a guitar in La Comuna 13

Source: https://verdadabierta.com/nosotras-somos-guerreras-la-verdad-la-paz-lideresas-la-comuna-13/galeria-comuna-13-19/

Figure 9: 'Bottle and Fishes' by George Barques

Source: https://www.tate.org.uk/art/artworks/braque-bottle-and-fishes-t00445

Figure 13-24: Murals in La Comuna 13 by various artists

Source: https://www.aboveusonlyskies.com/street-art-comuna-13-medellin/

Figures 25-27: 'Passage' Installation by Alfredo Baladron

Source: https://www.archdaily.com/916145/16-temporary-pavilions-that-reflect-on-public-space/5cc57a03284dd-

11a2b0001fd-16-temporary-pavilions-that-reflect-on-public-space-photo?next_project=no

Figures 28-30: 'City Thread' installation by SPORTS

Source: https://www.archdaily.com/910948/city-thread-sports?ad_medium=gallery 
Figures 31-33: Hosier Lane located in Melbourne, Australia

Source: https://www.lonelyplanet.com/australia/melbourne/attractions/hosier-lane/a/poi-sig/1370641/362494

Figures 34-35: Museum Langen Foundation by Tadao Ando Source: https://divisare.com/projects/382645-tadao-ando-lorenzo-zandri-museum-langen-foundation /Images by: Lorenzo Zandri

Figures 36-37: Joan Weill Centre for Dance by Iu + Bibliowicz Architects Source: Revealing Movement by Fred A. Berstein / Images by Eric Laignel

Figure 38-39: Y Ballet School by $y+M$

Source: https://www.archdaily.com/772533/y-ballet-school-y-plus-m?ad_medium=gallery / Images by Yohei Sasakura

Figures 40-42: MoMa Tower by Axis Mundi Architects

Source: https://axismundi.com/moma-tower

Figures 43-45: 'The Memory' by 23o5studio

Source: https://www.archdaily.com/788128/the-memory-23o5studio?ad_medium=gallery / Images by Quang Dam

Figures 46-48: Blaj Cultural Palace Refurbishment by Vlad Sebastian Rusu

Source: https://www.archdaily.com/790795/blaj-cultural-palace-refurbishment-vlad-sebastian-rusu-architecture-office?ad_medium=gallery

Figures 49-51: Olivetti Showroom by Carlo Scarpa

Source: https://www.archdaily.com/155074/ad-classics-olivetti-showroom-carlos-scarpa

Figures 52-54: 'El Pueblito Paisa', Historic landmark in Medellin, Colombia

Source: http://archivo.bitacoraeafit.com/el-pueblito-paisa-se-rehusa-a-dejar-atras-sus-raices/

Figures 55-57: House in Hiro by Suppose Design Office

Source: https://www.archdaily.com/51397/house-in-hiro-suppose-design-office/5008930028ba0d50da0010d7-house-in-hirosuppose-design-office-image?next_project=no

Figures 58-60: Andradas Apartment by OCRE arquitectura

Source: https://www.archdaily.com/946001/andradas-apartment-ocre-arquitetura?ad_medium=gallery /Images By Cristiano Bauce

Figures 61-63: Long House Apartment by Talita Nogueira Arquitectura Source: https://www.archdaily.com/946482/long-house-apartment-talita-nogueira-arquitetura?ad_medium=gallery / Images by Eduardo Macarios

Figures 64-66: AA8 Small House by Branco-DelRio Arquitectos Source: https://www.archdaily.com/942671/aa8-small-house-branco-delrio-arquitectos?ad_medium=gallery / Images by do mal o menos

Figures 67-69: Nakasone House by Escobedo Soliz Source: https://www.archdaily.com/936990/nakasone-house-escobedo-soliz/5e826819b35765979200016f-nakasone-house-escobedo-soliz-photo?next_project=no / Images by Ariadna Polo and Sandra Pereznieto 